University of Louisville

ThinkIR: The University of Louisville's Institutional Repository

Electronic Theses and Dissertations

$12-2017$

\title{
A novel approach to assess minimally invasive surgical device failure utilizing adverse event outcome severity and design complexity.
}

Marie K. Riggs

University of Louisville

Follow this and additional works at: https://ir.library.louisville.edu/etd

Part of the Biomedical Devices and Instrumentation Commons

\section{Recommended Citation}

Riggs, Marie K., "A novel approach to assess minimally invasive surgical device failure utilizing adverse event outcome severity and design complexity." (2017). Electronic Theses and Dissertations. Paper 2855. https://doi.org/10.18297/etd/2855

This Doctoral Dissertation is brought to you for free and open access by ThinkIR: The University of Louisville's Institutional Repository. It has been accepted for inclusion in Electronic Theses and Dissertations by an authorized administrator of ThinkIR: The University of Louisville's Institutional Repository. This title appears here courtesy of the author, who has retained all other copyrights. For more information, please contact thinkir@louisville.edu. 


\title{
A NOVEL APPROACH TO ASSESS MINIMALLY INVASIVE SURGICAL DEVICE FAILURE UTILIZING ADVERSE EVENT OUTCOME SEVERITY AND DESIGN COMPLEXITY
}

\author{
By \\ Marie K. Riggs \\ B.S., University of Louisville, 2013 \\ M. Eng., University of Louisville, 2014

\begin{abstract}
A Dissertation
Submitted to the Faculty of

for the Degree of

Doctor of Philosophy

in Mechanical Engineering

Department of Mechanical Engineering

University of Louisville

Louisville, KY
\end{abstract} \\ J.B. Speed School of Engineering of the University of Louisville \\ in Partial Fulfillment of the Requirements
}

December 2017 
(C) Copyright 2017 by Marie K. Riggs

All rights reserved 



\title{
A NOVEL APPROACH TO ASSESS MINIMALLY INVASIVE SURGICAL DEVICE FAILURE UTILIZING ADVERSE EVENT OUTCOME SEVERITY AND DESIGN COMPLEXITY
}

\author{
By \\ Marie K. Riggs \\ B.S., University of Louisville, 2013 \\ M.Eng., University of Louisville, 2014
}

A Dissertation Approved on

October $27^{\text {th }}, 2017$

by the following Dissertation Committee:

Dr. Gina Bertocci

Dr. Matt Bohm

Dr. Karen Bertocci

Dr. Peter Quesada

Dr. Thomas Berfield 


\section{DEDICATION}

I would like to dedicate this work to my parents, Brian and Teresa Riggs, as one small step towards living a life that gives justice to the sacrifices they made in order to provide me with so many invaluable opportunities.

This dissertation is also dedicated to Jeff Grinstead who motivated and supported me throughout this entire pursuit in addition to the many other ventures I pursued along the way. 


\section{ACKNOWLEDGEMENTS}

I would like to thank Dr. Matt Bohm. Without the opportunity and encouragement he provided, I would not have decided to pursue a doctorate degree nor would I have ever fully realized my capabilities in graduate research. Despite unforeseen obstacles and circumstances, he continued mentoring me to ensure my academic success, for which I will always be grateful.

I extend my gratitude to Dr. Gina Bertocci and Dr. Karen Bertocci for providing me with excellent guidance and feedback throughout my research. I thank Dr. Bikash Bhandari in the Department of Bioinformatics and Biostatistics for providing his time and expert advice regarding statistical analyses and interpretation throughout the various stages of my research project. I would also like to thank Dr. Peter Quesada and Dr. Thomas Berfield for serving on my dissertation committee.

I would like to acknowledge all the hard work my parents put forth throughout their life together and the challenges they overcame in order to make opportunities possible for so many others. I am very thankful for the vision and values they instilled in me from early on as part of a foundation for a successful life.

Finally, I would like to acknowledge and thank Jeff Grinstead. He encouraged me to pursue a $\mathrm{PhD}$ without hesitation. The past three years would have been much more difficult without his motivation, inspiration, and support. 


\title{
ABSTRACT \\ A NOVEL APPROACH TO ASSESS MINIMALLY INVASIVE SURGICAL DEVICE FAILURE UTILIZING ADVERSE EVENT OUTCOME SEVERITY AND DESIGN COMPLEXITY
}

\author{
Marie K. Riggs
}

October $27^{\text {th }}, 2017$

Medical device failure and misuse have the potential to cause serious injury and

death. Given the intricate nature of the instruments utilized specifically in minimally invasive surgery (MIS), users and manufacturers of surgical devices share a responsibility in preventing user error and device failure. A novel approach was presented for the evaluation of minimally invasive device failures, which involved assessing the severity of adverse event outcomes associated with the failures modes and investigating aspects of the devices' design that may contribute to failure. The goals of this research were to 1) characterize the design attributes, failure modes, and adverse events associated with minimally invasive surgical devices and 2) describe the relationship between minimally invasive surgical device design complexity and the severity of adverse events. The types of failure modes, phases of operation in which failure occurs, severity of adverse event outcomes, and design complexity associated with four minimally invasive surgical devices were determined.

An association was shown to exist between phases of surgical device operation and the severity of outcomes that occur in each phase $(p<0.05)$. Across both device types, the majority of failure occurred during execution of the devices' main function, 
which involved securing and transecting tissue. The least amount of failures occurred during the results and post-op phase of operation; however, the failures that occurred during this phase resulted in the highest average outcome severity. The endoscopic staplers assessed resulted in overall higher average outcome severities relative to that of the tissue sealers.

The methods employed are the first to evaluate medical device design, function, and failure outcomes from a complexity perspective. While statistical conclusions regarding the overall research goal could not be drawn, heuristic methods support development of the approach presented. The work herein assists the enhancement of risk awareness and prevention techniques and serves as a contribution to filling the knowledge gap regarding device use and failure outcomes. Bridging the gap between surgeons and engineers is crucial to the successful implementation and evaluation of new technology in the operating room, which was an essential component of this research. 
TABLE OF CONTENTS

PAGE

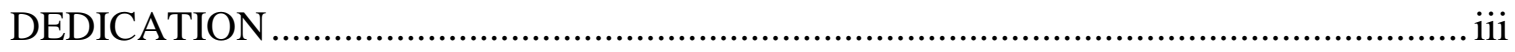

ACKNOWLEDGEMENTS ................................................................................... iv

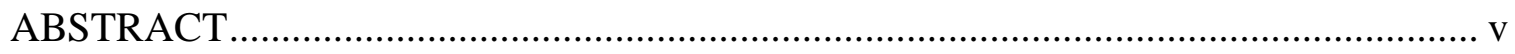

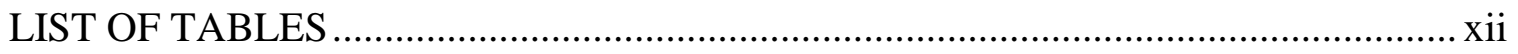

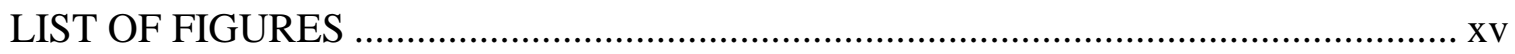

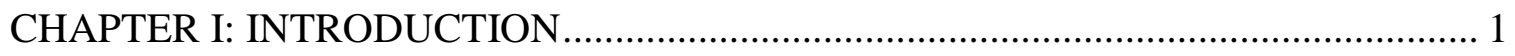

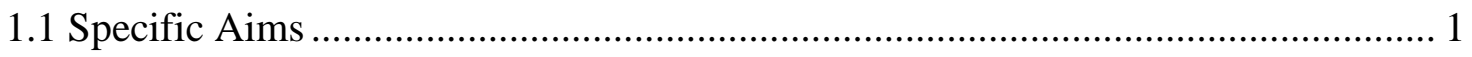

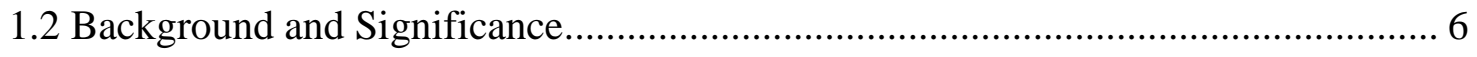

1.2.1 Minimally Invasive Surgical Devices.............................................................. 6

1.2.1.1 Minimally Invasive Surgical Device Overview......................................... 6

1.2.1.2 Endoscopic Stapler Overview ............................................................... 7

1.2.1.3 Energy-Based Tissue Sealer Overview .................................................... 8

1.2.2 Clinical Variables and User Knowledge Gap................................................. 10

1.2.2.1 Clinical Knowledge Gap ........................................................................ 10

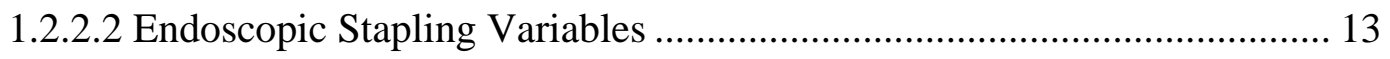

1.2.2.3 Energy-Based Tissue Sealing Variables ................................................. 15

1.2.3 Medical Device Regulation and Reporting ................................................... 16 
1.2.3.1 Medical Device Regulation.................................................................... 16

1.2.3.2 Medical Device Reporting and Recalls.................................................. 21

1.2.3.3 Limitations of Medical Device Reporting ............................................. 22

1.2.4 Surgical Device Failure Mode Studies .............................................................. 23

1.2.4.1 Endoscopic Stapler Failure Mode Studies ................................................ 23

1.2.4.2 Energy-Based Tissue Sealer Failure Mode Studies ................................ 25

1.2.4.3 Limitations of Failure Mode Studies ....................................................... 27

1.2.5 Severity Grading Methods.......................................................................... 28

1.2.5.1 Overview of Current Methods .......................................................... 28

1.2.5.2 Limitations of Severity Grading Methods ………………........................ 32

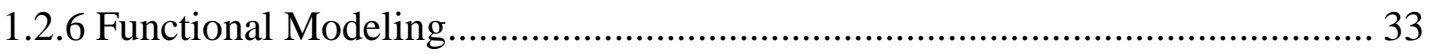

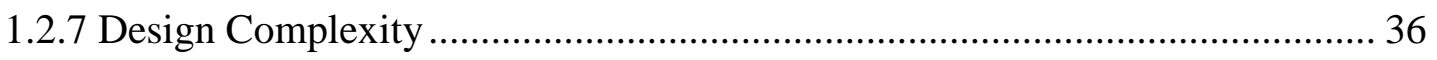

1.2.7.1 Surgical Device Design Complexity ...................................................... 36

1.2.7.2 Generalized Complexity Index ……………....................................... 39

1.2.7.3 Connectivity Algorithm ........................................................................ 41

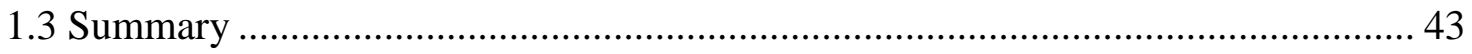

CHAPTER II: SURGICAL DEVICE FAILURE MODES ........................................... 46

2.1 Failure Mode Study Overview ………………................................................. 46

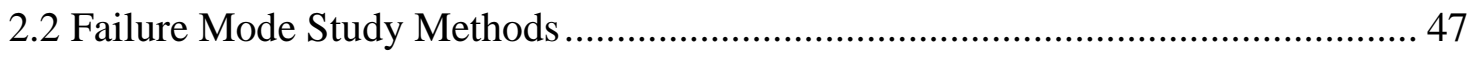

2.2.1 FDA Database Query.............................................................................. 47

2.2.1.1 Endoscopic Stapler FDA Database Query ............................................. 48

2.2.1.2 Energy-Based Tissue Sealer FDA Database Query.................................. 48

2.2.1.3 Failure Mode Study Sample ................................................................... 50 
2.2.2 Failure Mode Study Data Analysis ............................................................. 51

2.2.2.1 Failure Mode Data Analysis ................................................................ 51

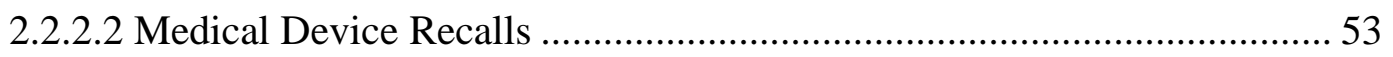

2.3 Failure Mode Study Results ........................................................................... 53

2.3.1 Manufacturer Narrative Excerpts ................................................................. 53

2.3.2 Endoscopic Stapler Failure Modes and Recalls ............................................. 55

2.3.3 Energy-Based Tissue Sealer Failure Modes and Recalls .................................. 62

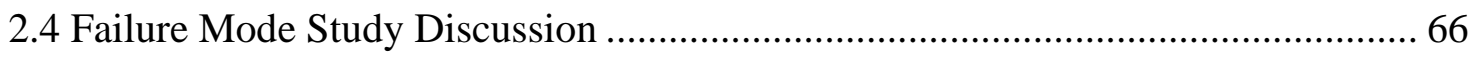

2.4.1 Endoscopic Stapler Failure Mode Discussion ................................................ 66

2.4.2 Energy-Based Tissue Sealer Failure Mode Discussion...................................... 75

2.4.3 Manufacturer Narratives and Event Descriptions ......................................... 85

2.5 Failure Mode Study Limitations and Future Work ………………...................... 86

CHAPTER III: SEVERITY OF ADVERSE EVENT OUTCOMES ............................... 90

3.1 Adverse Event Outcome Severity Study Overview …………............................ 90

3.2 Adverse Event Outcome Severity Methods ............................................................ 91

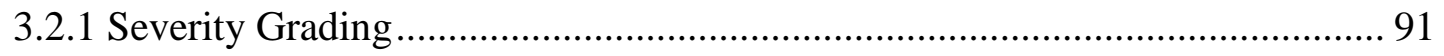

3.2.1 Adverse Event Outcome Severity Data Analysis ............................................ 92

3.3 Adverse Event Outcome Severity Results .......................................................... 94

3.3.1 Severity of Failure Mode Outcomes Associated with Endoscopic Staplers .... 94

3.3.2 Severity of Failure Mode Outcomes Associated with Tissue Sealers ............... 98

3.3.3 Severity of Failure Mode Outcomes Associated with Both Device Types .... 103

3.4 Adverse Event Outcome Severity Discussion........................................................ 107

3.4.1 Endoscopic Stapler Adverse Event Outcome Severity.................................... 108 
3.4.2 Energy-Based Tissue Sealer Adverse Event Outcome Severity 114

3.4.3 Surgical Device Adverse Event Outcome Severity 119

3.5 Outcome Severity Study Limitations and Future Work 124 CHAPTER IV: DEVICE DESIGN COMPLEXITY SCORE 127

4.1 Complexity Study Overview 127

4.2 Complexity Scoring Methods. 128

4.2.1 MIS Devices Obtained for the Complexity Analysis 129

4.2.1.1 Endoscopic Staplers 129

4.2.1.2 Energy-Based Tissue Sealers 130

4.2.2 Connectivity Algorithm 131

4.3 Complexity Results 134

4.3.1 Functionality, Reverse Engineering, and DSMs 134

4.3.1.1 Endoscopic Stapler Function and DSM 134

4.3.1.2 Energy-Based Tissue Sealer Function and DSM 141

4.3.2 Complexity Scores. 148

4.4 Complexity Discussion 150

4.5 Complexity Study Limitations and Future Work 157 CHAPTER V: OUTCOME SEVERITY AND DEVICE DESIGN COMPLEXITY .... 160

5.1 Outcome Severity and Design Complexity Study Overview 160

5.2 Severity and Complexity Methods 161

5.3 Severity and Complexity Results 162

5.4 Severity and Complexity Discussion 165

5.5 Severity and Complexity Study Limitations and Future Work 170 
CHAPTER VI: SUMMARY AND CONCLUSIONS .......................................... 172

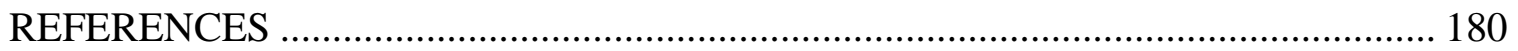

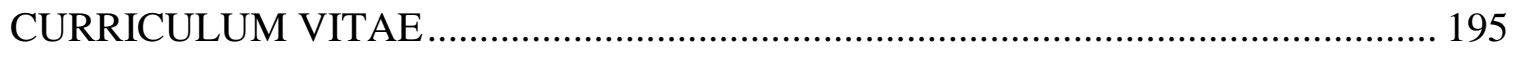




\section{LIST OF TABLES}

PAGE

Table 1. FDA device classification (Yock, Zenios et al. 2015).................................... 18

Table 2. CTCAE v3.0 category-specific grading (NIH 2003)................................... 31

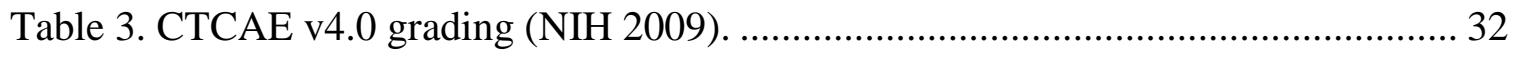

Table 4. Description of variables in the generalized complexity index......................... 40

Table 5. Connectivity algorithm (Ameri, Summers et al. 2008). ................................. 42

Table 6. FDA product code search for endoscopic staplers....................................... 48

Table 7. FDA product code search for bipolar and ultrasonic devices......................... 49

Table 8. Number of death and injury reports downloaded from MAUDE database....... 50

Table 9. Number of death and injury reports included in the sample for analysis. ......... 51

Table 10. Endoscopic stapler phases and failure modes for ETS ( $\mathrm{n}=111$ failures derived

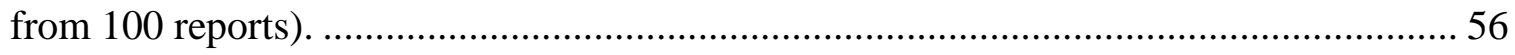

Table 11. Endoscopic stapler phases and failure modes for the Endo GIA ( $n=113$ failures

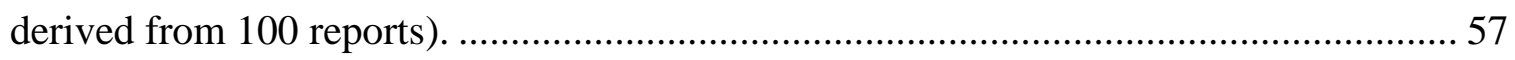

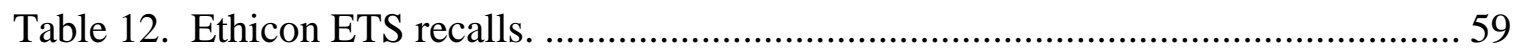

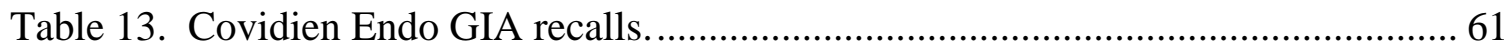

Table 14. Tissue sealer phases and failure modes for the Olympus Thunderbeat $(n=81$

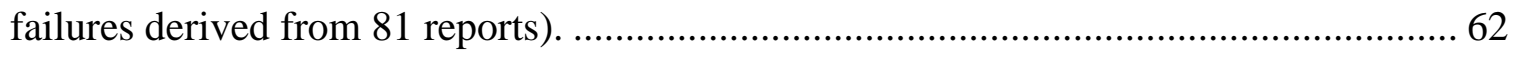

Table 15. Tissue sealer phases and failure modes for the Ethicon Harmonic Ace $(n=102$

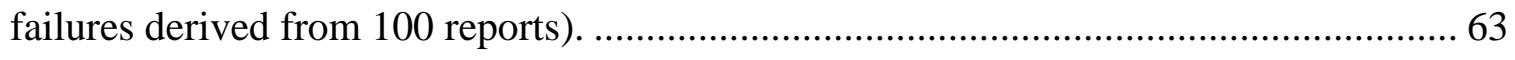

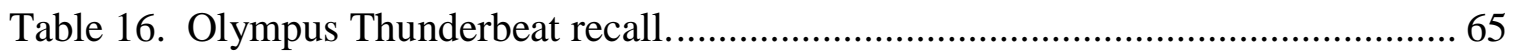


Table 17. Ethicon Harmonic Ace recalls.

Table 18. Severity of failure mode outcomes associated with the ETS endoscopic stapler ( $\mathrm{n}=111$ failures derived from 100 reports).

Table 19. Severity of failure mode outcomes associated with the Endo GIA endoscopic

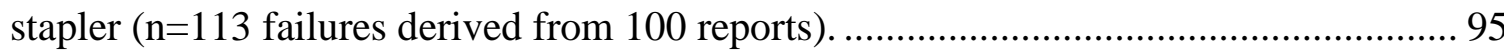

Table 20. Endoscopic stapler failure frequencies (proportions) and average severity of outcomes per phase.

Table 21. Endoscopic stapler failure outcome severity frequencies per phase of operation. 96

Table 22. Endoscopic stapler average severity per phase of operation and overall percentage of failure outcomes with severity of 3 or higher. 96

Table 23. Endoscopic stapler percentage of outcomes with severity 3 or higher relative to the number of outcomes that occurred during each phase of operation. 97

Table 24. Endoscopic stapler cross tabulation table. 98

Table 25. Chi-Squared tests SPSS output for endoscopic stapler outcome severity and phase of operation. 98

Table 26. Severity of failure modes associated with the Thunderbeat tissue sealer $(n=81$ failures derived from 83 reports). 99

Table 27. Severity of failure mode outcomes associated with the Harmonic Ace tissue sealer ( $\mathrm{n}=102$ failures derived from 100 reports). 100

Table 28. Tissue sealer failure frequencies (proportions) and average outcome severity per phase. 100

Table 29. Tissue sealer failure outcome severity frequencies per phase of operation... 101 Table 30. Tissue sealer average severity per phase of operation and overall percentage of failure outcomes with severity of 3 or higher. 101

Table 31. Tissue sealer percentage of outcomes with severity 3 or higher relative to the number of outcomes that occurred during each phase of operation. 102 
Table 32. Tissue sealer cross tabulation table. 103

Table 33. Chi-Squared tests SPSS output for tissue sealer outcome severity and phase of operation. 103

Table 34. Combined failure outcome severity frequencies and proportions. 104

Table 35. Surgical device average severity per phase of operation and overall percentage of failure outcomes with severity of 3 or higher. 104

Table 36. Surgical device percentage of outcomes with severity 3 or higher relative to the number of outcomes that occurred during each phase of operation. 105

Table 37. Overall percentage of failure outcomes with severity of 3 or higher relative to the number of outcomes that occurred during each phase of operation. 105

Table 38. Surgical device cross tabulation table. 106

Table 39. Chi-Squared tests SPSS output for surgical device outcome severity and phase of operation. 107

Table 40. Number of components and complexity scores per device. 149

Table 41. Functional model parameters and complexity score. 149

Table 42. Sample percentages for regression variables. 162

Table 43. Design complexity scores and average severities per device type. 163

Table 44. Functional model complexity scores and average severities per device type. 163 Table 45. Model fitting information for ordinal regression. 163

Table 46. Ordinal regression parameter estimates with respect to a complexity score of 478 164

Table 47. Outcome severity odds ratios and significance with respect to each complexity score. 165 


\section{LIST OF FIGURES}

PAGE

Figure 1. Black box model (Nagel, Bohm et al. 2015) ………………........................ 35

Figure 2. Functional modeling chains (Nagel, Bohm et al. 2015).................................. 35

Figure 3. Functional model (Nagel, Bohm et al. 2015).................................................. 36

Figure 4. Proportion of failures per phase and device brand (ETS n=113, Endo GIA

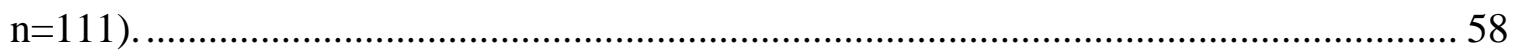

Figure 5. Proportion of failures per phase and device brand (Thunderbeat $n=81$,

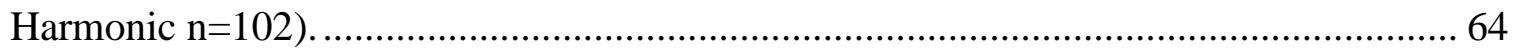

Figure 6. Distribution of severity per phase of operation associated with endoscopic

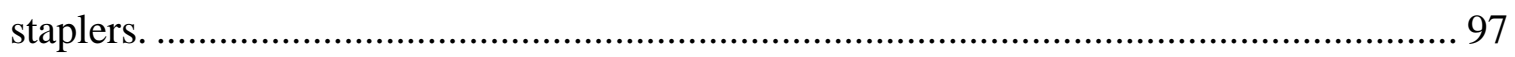

Figure 7. Distribution of severity per phase of operation associated with tissue sealers.

Figure 8. Distribution of severity per phase of operation............................................. 106

Figure 9. The Covidien Endo GIA ${ }^{\mathrm{TM}}$ Universal stapler and the Covidien Endo GIA ${ }^{\mathrm{TM}}$

Universal Roticulator ${ }^{\mathrm{TM}}$ reload attachment. .................................................................. 129

Figure 10. Ethicon Endo-Surgery Endopath ${ }^{\circledR}$ ETS Endoscopic Articulating Linear

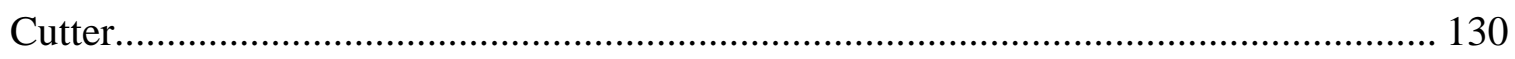

Figure 11. Olympus Thunderbeat Surgical Tissue Management System....................... 131

Figure 12. Ethicon Harmonic Ace tissue sealer............................................................. 131

Figure 13. Bipartite graph (entity relationship graph) (Summers and Ameri 2008)..... 133

Figure 14. Ethicon ETS loaded jaw..................................................................... 135 
Figure 15. Distal Covidien Endo GIA reload.

Figure 16. Ethicon ETS handle with triggers and release button. 136

Figure 17. Covidien Endo GIA handle with trigger and return knobs.

Figure 18. Endoscopic stapler functional model.

Figure 19. Ethicon Endo-Surgery Endopath® ETS Endoscopic Articulating Linear

Cutter exploded view.

Figure 20. Covidien Endo GIA ${ }^{\mathrm{TM}}$ Universal stapler and the Covidien Endo GIA ${ }^{\mathrm{TM}}$

Universal Roticulator ${ }^{\mathrm{TM}}$ reload exploded view. 139

Figure 21. Ethicon ETS stapler DSM subset. 140

Figure 22. Covidien Endo GIA stapler DSM subset. 141

Figure 23. Olympus Thunderbeat activation hand switches. 142

Figure 24. Ethicon Harmonic Ace activation hand switches. 143

Figure 25. Ethicon Harmonic Ace ultrasonic jaw (active blade). 144

Figure 26. Olympus Thunderbeat ultrasonic and bipolar jaw (active probe tip). 144

Figure 27. Energy-based tissue sealer functional model. 145

Figure 28. Olympus Thunderbeat exploded view 146

Figure 29. Ethicon Harmonic Ace exploded view. 146

Figure 30. Olympus Thunderbeat DSM subset. 147

Figure 31. Ethicon Harmonic Ace DSM subset. 148

Figure 32. Branching of mechanisms and designs from functional requirements....... 150 


\section{CHAPTER I}

\section{INTRODUCTION}

\section{$\underline{1.1 \text { Specific Aims }}$}

The progression of medical devices contributes to countless saved lives by enabling the alleviation of pain, conquering of disability, and sustainment of life. However, device failure and misuse have the potential to cause serious injury and death. The Institute of Medicine (IOM) estimated that 1.3 million serious injuries to Americans are caused by medical devices each year (Rados 2003). Further, it was stated that more deaths occur due to medical errors occurring in hospitals than from AIDS, breast cancer, or motor vehicle accidents in a given year (Rados 2003). More than 2,000 device-related deaths and more than 200,000 reports of device-related injuries and malfunctions are reported to the FDA every year (Mouzoon and Carome 2012). Many adverse events are attributed to user error; though, the rising trends in the number of recalls indicate that manufacturing error and design flaws are also contributing factors. In the fiscal year 2011, there were 1201 recalls for moderate- and high-risk devices, which is more than double the number of recalls in 2007 (Mouzoon and Carome 2012). This substantial increase was not paralleled by an increase in the number of new product applications submitted to the FDA, which gives reason for investigation into manufacturing and design defects (Mouzoon and Carome 2012). 
Rapid technological advancement has allowed the development of highly complex, intricate medical devices, which is especially notable in the evolution of devices used for minimally invasive surgery (MIS) (Janetschek, Bagheri et al. 2003, Feldman, Fuchshuber et al. 2012, Chekan and Whelan 2014). MIS has become more applicable to an increasing number and variety of procedures and in many cases has become the preferred technique over open procedures (Belli, Fantini et al. 2006, Hu, Gu et al. 2009, Zingg, McQuinn et al. 2009, Biere, van Berge Henegouwen et al. 2012, Swanson, Meyers et al. 2012). Numerous commendable user and patient benefits are associated with MIS, yet evidence of a knowledge gap in surgeons' understanding of surgical devices has the potential to negatively impact the clinical outcome of operations (McColl, Karmali et al. 2009, Feldman, Fuchshuber et al. 2012, Feldman, Brunt et al. 2013, Chekan and Whelan 2014, Madani, Watanabe et al. 2016). For example, a study survey given to a target test audience demonstrated the consequences of rapid device advancement as it revealed only $28 \%$ of respondents considered themselves "experts" in the area of surgical energy-based devices used in MIS (Feldman, Brunt et al. 2013). A knowledge gap in users' understanding of devices combined with the potential for device failure is of great concern as it can result in detrimental effects on patient and procedural outcomes.

At any stage of a minimally invasive procedure user or device failure has the potential to cause catastrophic consequences, such as severe hemorrhage and conversion to open surgery, which puts patients' lives at risk. General surgical errors account for approximately half of all adverse events and up to $13 \%$ of hospital deaths (McCrory, LaGrange et al. 2014). Investigation into device class-specific adverse events reveals that 
the rate of injuries from surgical energy has been estimated as one to two per 1,000 operations (Nduka, Super et al. 1994, Overbey, Townsend et al. 2015). This rate of injury is relatively low; however, considering more than 50 million inpatient procedures are performed in the United States each year, continuous and additional efforts are necessary in order to reduce these adverse outcomes in the operation setting (Overbey, Townsend et al. 2015).

Given the intricate nature of MIS and the instruments utilized, users and manufacturers of surgical devices share a responsibility in preventing user error and device failure. In an investigation into casual factors for medication and medical device related adverse clinical incidents, medical equipment breakage and failure was found to be the most common precursor event for medical device related incidents (46\%), and $22.5 \%$ of medical device related incidents occurred intraoperatively (Mitchell, Williamson et al. 2015). Complexity of a surgical device's design has the potential to inhibit a user's understanding of proper operation and techniques for failure prevention; it could also impact proper device functionality. Understanding the complexity of surgical devices can provide valuable insight into specific mechanisms of failure and a means to implement countermeasures against undesired outcomes in the surgical setting. Further, risk awareness and prevention techniques can be enhanced via an assessment of the severity of adverse event outcomes that result from failure.

The long term goal was to facilitate intraoperative safety and prevent patient injury and death caused by adverse events associated with minimally invasive surgical devices. The specific goals of this research were to 1) characterize the design attributes, failure modes, and adverse event outcomes associated with minimally invasive surgical 
devices and 2) describe the relationship between minimally invasive surgical device design complexity and the severity of adverse events. The research presented supplies users and manufacturers with insight regarding phases of operation and mechanisms responsible for the failures related to surgical devices in addition to the nature of the associated adverse event outcomes. The following specific aims were established as steps to achieve the research goal:

1. Describe the types of failure modes shown to be associated with endoscopic staplers and energy-based tissue sealers used in MIS based on reported adverse events.

2. Describe the severity of adverse event outcomes that are associated with the failure modes of endoscopic staplers and energy-based tissue sealers.

3. Characterize the design complexity of endoscopic staplers and energy-based tissue sealers used in MIS using device component decomposition and functional modeling.

4. Describe the relationship between minimally invasive surgical device design complexity and the severity of adverse event outcomes associated with endoscopic staplers and energy-based tissue sealers.

H1: As minimally invasive surgical device design complexity increases, the severity of adverse event outcomes associated with the device also increases.

5. Develop a tool to predict the likelihood of severe adverse events associated with minimally invasive surgical devices based on design complexity. 
The specific aims were addressed by carrying out the following research components. The first component involved characterizing the nature and frequency of failure modes associated with two relatively diverse classes of minimally invasive surgical devices. Characterization was achieved through data collection and analysis of reports retrieved from the United States (US) Food and Drug Administration (FDA) Manufacturer and User Facility Device Experience (MAUDE) database, which was outlined in Chapter II. The next component assessed the severity of the adverse event outcomes associated with the failure modes via application of the Common Terminology Criteria for Adverse Events (CTCAE) v4.0 grading scale as described in Chapter III. Chapter IV presents the third component, which consisted of reverse engineering four minimally invasive surgical devices and determining a design complexity score based on an existing complexity measure. Chapter $\mathrm{V}$ discusses the final component of this research, which was to utilize the average outcome severity and complexity score associated with each surgical device in order to determine the relationship between the two variables. An extension of this component involved exploring the viability of an outcome severity predictive tool based on complexity by performing ordinal regression, which would be applied in the early design stages to mitigate potential design-related adverse events.

Chapter VI concludes with a summary of results and discussion. Through testing of the hypothesis, the variables investigated associated with complexity and outcome severity can potentially enhance engineers' abilities to prevent or reduce the severity of surgical device failure mode outcomes. A novel approach to assessing minimally invasive device failures and adverse event outcomes was presented, which included 
evaluating medical device design, function, and failure outcomes from a design complexity perspective.

\subsection{Background and Significance}

\subsubsection{Minimally Invasive Surgical Devices}

\subsubsection{Minimally Invasive Surgical Device Overview}

A medical device is defined as an instrument used for diagnosis, treatment, or prevention of a disease, injury, or other condition that is not a drug, biologic or food (Rados 2003). Types of medical devices range from simplistic tongue depressors to complex surgical tools. Significant medical device innovation has emerged and contributes specifically to minimally invasive surgical techniques. Minimally invasive surgery (MIS) has been applied to an increasing number and variety of procedures and in

many cases has become the preferred technique over open procedures (Belli, Fantini et al. 2006, Hu, Gu et al. 2009, Zingg, McQuinn et al. 2009, Biere, van Berge Henegouwen et al. 2012, Swanson, Meyers et al. 2012). MIS has transformed many medical procedures and offers various advantages compared to conventional surgery, such as reduced postoperative pain, faster recovery, reduction in intraoperative bleeding, and reduced postoperative complications (Belli, Fantini et al. 2006, Hu, Gu et al. 2009, Zingg, McQuinn et al. 2009, Handy, Asaph et al. 2010, Biere, van Berge Henegouwen et al. 2012, Irwin and Wong 2012). The goal of medical treatment is further realized with the application of MIS, which is "to minimize harm to patients and maximize the natural self-healing power for fighting against the disease" (Wang 2015). The medical devices 
and accompanying tools for such procedures allow a decrease in the size of incisions required for operation, which in turn spares the surrounding tissue (Irwin and Wong 2012).

Procedurally innovative endoscopic approaches have been enabled through the improved design of surgical instrumentation. Miniaturization of surgical instruments, such as forceps, cutting tools, electronic sensors, drug delivery systems, and lighting technology, facilitates access to regions of the body that once had to be opened entirely. This research focuses on the following types of devices for MIS: endoscopic staplers and energy-based tissue sealers.

\subsubsection{Endoscopic Stapler Overview}

The use of endoscopic stapling devices is increasingly being applied to more wide-ranging open and MIS procedures (Deng, Meng et al. 2002, Janetschek, Bagheri et al. 2003, Belli, Fantini et al. 2006, Robert, Poncet et al. 2011, Hassouna and Manikandan 2012, Kwazneski, Six et al. 2012). Endoscopic staplers deploy either purely mechanical or powered mechanisms to simultaneously staple and transect target tissue during the

firing sequence. Stapling occurs as individual straight metal staples are released from the cartridge, travel through the compressed tissue, and typically bent into a "B" shape upon contact with a hard anvil pocket on the opposite side (Mery, Shafi et al. 2008).

Alterations of the pocket design and resulting staple shape have been initiated on more recent device designs in attempt to improve hemostasis. Stable anastomosis relies on the integrity of the staple line, which directly relates to the amount of tissue compression (Chekan and Whelan 2014). Intrinsic biomechanical properties of tissue vary along with 
the tissue types and thickness. Tissue properties determine both the proper compressive force and optimal compression time before firing the device (Chekan and Whelan 2014). Device performance depends heavily on the proper shape formation of staples, integrity of the staple line, smooth division of target tissue, and proper overall mechanical function through all stages of device use.

Endoscopic staplers are one of many instruments that have been developed and approved for use in open surgery and MIS. For various procedures, endoscopic staplers are often used to execute the most difficult and anxiety-provoking portions of the operation, such as ligation and division of vascular structures (Deng, Meng et al. 2002). These devices are utilized in procedures such as lobectomy, nephrectomy, gastrectomy, gastric bypass, appendectomy, hepatectomy, and video-assisted thoracic surgery (VATS),

to name a few. The successful creation of an anastomosis from an optimal staple line can be achieved by applying the proper size cartridge and corresponding staple height based on the tissue's thickness and mechanical properties. Performance of endoscopic staplers relies on an optimal compression force that allows for proper tissue approximation, yet spares tissue from shearing or injury (Mery, Shafi et al. 2008).

\subsubsection{Energy-Based Tissue Sealer Overview}

The energy-based devices of interest seal vessels and transect tissue through utilization of bipolar and ultrasonic technology. Devices within this category are relied upon to create a hemostatic seal as a result of denaturation of collagen and elastin in vessel walls (Aytan, Nazik et al. 2014). Two electrodes are contained within a bipolar electrosurgical device, between which the tissue is grasped. Current passes through the 
tissue between these electrodes causing distribution of thermal energy and coagulation of vessel walls. Advanced bipolar devices deliver pulses of electrical energy, which allows the tissue to cool throughout activation in attempt to reduce tissue sticking on the electrode. Such devices also rely on computer-controlled feedback response systems in order to adjust the current and voltage based on tissue impedance and temperature (Lyons and Law 2013). Tissue impendence and temperature is regulated via computer-controlled feedback response systems, and positive temperature coefficient (PTC) technology may be incorporated to minimize undesired thermal spread surrounding the application site. The electrodes of bipolar devices are efficient for sealing vessels, and a cutting device, such as a retractable blade, is built within to allow a mechanism for transection of tissue. Controlling the frequency, intensity, and duration of electrosurgical energy delivered to the tissue provides surgeons with the option to cauterize, coagulate, and/or reduce bleeding (Johnson, Couture et al. 2007).

Ultrasonic devices result in a similar tissue effect as produced by bipolar devices, yet it is achieved via ultrasonic vibrations. Electrical energy is converted to mechanical and thermal energy by means of the vibrations in order to both seal and transect vessels. Bipolar and ultrasonic devices involve conversion of electrical energy into mechanical and thermal energy, yet the methods vary as described. To achieve the necessary tissue effects, bipolar devices convert energy via intracellular frictional effects, whereas the frictional force generated by ultrasonic devices results in extracellular heating of tissue followed by intracellular heating (Lyons and Law 2013). The effectiveness of these devices relies on the pressure applied to the vessel and the gap distance between the electrodes; such mechanical properties must be constantly maintained at the proper 
setting based on the vessel thickness (Johnson, Couture et al. 2007). The energy-based devices are utilized in various laparoscopic procedures that include, but not limited to, colectomy, hysterectomy, liver resection, lobectomy, and pancreatectomy.

\subsubsection{Clinical Variables and User Knowledge Gap}

\subsubsection{Clinical Knowledge Gap}

The technological advancements that contribute to the evolution of devices for MIS lead to the development of new and modified surgical techniques. A consequence of the rapid rate of the technological advancement is a lack in surgeons' knowledge of surgical devices, which has been referred to as a "knowledge gap" in surgeons' understanding of how devices interact with tissue. Since many surgeons are not aware of the tissue handling characteristics and limitations of new or reengineered devices, the knowledge gap can impact the clinical outcome of operations (Feldman, Fuchshuber et al. 2012, Feldman, Brunt et al. 2013, Sankaranarayanan, Resapu et al. 2013, Chekan and Whelan 2014). Adverse events have been shown to be attributed to both user error as well as device-related errors. Therefore, continuous training and education for surgeons and device design improvements by manufacturers are imperative (Zingg, McQuinn et al. 2009, Madani, Watanabe et al. 2014, Wang 2015, Madani, Watanabe et al. 2016, Watanabe, Kurashima et al. 2016).

In a study of laparoscopic linear cutting stapler failure, less than $0.3 \%$ of the incidences were primarily related to device failure when cases of user error were excluded and the use of multiple staplers and cartridges was included (Deng, Meng et al. 2002). In a study by Chen et al., the incidence of primary device failure was estimated to 
be less than 0.2\% (Chan, Bishoff et al. 2000). These outcomes demonstrate the criticality of surgeon experience, knowledge, and vigilance.

Despite the training programs offered by manufacturers and the instructions for device use, more resources are necessary (Parker 2010). McColl et al. studied the baseline knowledge of endoscopic staplers possessed by surgical residents and found a deficiency of knowledge, especially in junior residents (McColl, Karmali et al. 2009). Manufacturer-offered courses that detail the function and operation of special surgical instrumentation are widely available for attending physicians; however, trainees are typically not offered such courses. Surgeons are often relied upon to train the residents about each specialized surgical device in an operating room setting, which can result in an increase in procedure time and costs (Bridges and Diamond 1999, Lavernia, Sierra et al. 2000, Farnworth, Lemay et al. 2001). Educational techniques must evolve for physicians and residents in response to technological advancements of surgical instrumentation in order to fill the knowledge gap and prevent adverse operative outcomes.

Surgical energy devices are a common tool used by surgeons and in many cases are used on a daily basis (Watanabe, Kurashima et al. 2016). Surveys results given to a target test audience revealed that only $28 \%$ of respondents considered themselves "experts" in the area of surgical energy-based devices (Feldman, Brunt et al. 2013). Additionally, of 48 Society of American and Gastrointestinal and Endoscopic Surgeons (SAGES) leaders who took a pretest examination of energy use in surgery, the median percent of correct answers was 59\% (Feldman, Fuchshuber et al. 2012). The test revealed that $13 \%$ did not know that thermal injury can spread outside of the bipolar 
instrument jaws, and $31 \%$ could not identify the device that would be the least likely to interfere with a pacemaker (Feldman, Fuchshuber et al. 2012). After informing the participants of the correct answers, a different post-test completed by 25 of the participants resulted in a median of $90 \%$ correct answers, revealing the potential of a formal curriculum to help fill the knowledge gap (Feldman, Fuchshuber et al. 2012). A standard curriculum is typically not available outside of the training offered by the device manufacturers.

Due to the potential knowledge gap suggested in regards to surgical energy-based devices, SAGES created a curriculum called the Fundamental Use of Surgical Energy (FUSE) to address the safety and usage concerns (Feldman, Fuchshuber et al. 2012, Feldman, Brunt et al. 2013). FUSE was launched in 2012 and includes content such as fundamentals of electrosurgery, monopolar devices, bipolar devices, endoscopic devices, and ultrasonic energy systems. Initial studies have shown that a formal curriculum in specific surgical medical devices, such as FUSE, can decrease the knowledge gap and consequentially reduce complications (Feldman, Brunt et al. 2013, Overbey, Townsend et al. 2015, Madani, Watanabe et al. 2016, Watanabe, Kurashima et al. 2016). Yet, combined efforts from medical, design, technology, and manufacturing domains are necessary.

Five strategies have been identified using a system approach by Dankelman and Grimbergen aimed at the reduction of MIS errors: (1) reduce complexity, (2) standardize procedures, (3) implement checklists, (4) improve the quality and standardization of instruments and equipment and (5) training (Dankelman and Grimbergen 2005). The overall approach calls for the elimination of unnecessary and inefficient interactions and 
processes in addition to a standardization of procedures and equipment (Flin, O'Connor et al. 2008). Errors within complex systems can be due to a confliction between the work system and operator/user capabilities (or lack thereof) (van Det, Meijerink et al. 2009). Also, in order to reduce complexity, a method must be applied in order to identify and quantify it.

\subsubsection{Endoscopic Stapling Variables}

Surgeons are faced with multiple complex variables upon implementation of their choice of stapling technique. Examples of the variables associated with endoscopic stapling devices include staple height associated with cartridge or reload selection, compression time, buttress usage, overlapping staple lines, and tissue properties. Intrinsic biomechanical properties of tissue vary along with the tissue types and thickness. Performance of endoscopic staplers relies on an optimal compression force that allows for proper tissue approximation, yet spares tissue from shearing or injury (Mery, Shafi et al. 2008). Mery et al. profiled endoscopic staplers through a series of leak pressure tests and found staple height was an important determinant of leak pressure, overlap did not affect leak pressure, and buttressing improved all types of staple lines (Mery, Shafi et al. 2008). Buttressing refers to thin sheets that are placed on one or both sides of the target tissue in attempt to supply additional support and hemostasis (Mery, Shafi et al. 2008). Less bleeding from staple lines has been associated with buttressing, yet it can cause visualization issues and interference with repeated staple firing (Angrisani, Lorenzo et al. 2004). 
Overlapping staple lines has been shown to attribute to adverse event outcomes and can result in dulling of the blade and incomplete staple lines accompanied by a difficulty in firing (Mery, Shafi et al. 2008). Though, certain procedures call for overlapping staple lines in order to complete a transection, such as the creation of a gastric pouch during gastric bypass. That is yet another factor that the surgeon must consider during his/her choice of endoscopic stapling technique.

A user's choice of stapler and staple height depend heavily on the tissue type. Body tissue ranges in thickness and the dimensions vary based on age, anatomical location, sex, disease state, structure, and patient medications (Chekan and Whelan 2014). Tissue consists of both solid and liquid, and its intrinsic properties must be considered, such as protein content and metabolic factors (Chekan and Whelan 2014). Firing should be initiated on elongated tissue with a decreased quantity of fluid, which is achieved by optimal compression times. When choosing a stapler, the user must consider these tissue-specific properties, which also includes vascularity of the structure, tension, and likelihood of variable tissue thickness (Chekan and Whelan 2014).

Many adverse events are attributed to user error; even so, manufacturers have a responsibility to recognize and address any overwhelming confusion or challenges faced by surgeons during use. Also, the number of recalls associated with endoscopic staplers indicates device error shares responsibility (Deng, Meng et al. 2002, Kwazneski, Six et al. 2012). Filling the knowledge gap requires consistent reporting of adverse events to increase awareness, providing up-to-date educational techniques for physicians and residents, and ensuring the development of safe, reliable, and user-friendly devices. 


\subsubsection{Energy-Based Tissue Sealing Variables}

Energy-based devices in particular have increased in the rate of technological evolution and complexity. Delicate, intricate procedures combined with the incorporation of electrical energy and heat generation create high-pressure environments in operating rooms. In addition to the wide range of device options and features, such an operational setting can induce devastating complications. According to the Emergency Care Research Institute (ECRI), surgical burns and fires are listed in the top 10 health technology hazards for 2012 (Feldman, Fuchshuber et al. 2012).

The hazards of electrosurgery must be monitored by the surgeon and staff. Surgical smoke produced during tissue diathermy can be potentially hazardous to staff and patients. Based on chemical analysis, $5 \%$ of the surgical smoke consists of potentially harmful content, such as hydrocarbons and nitriles (Gallagher, Dhinsa et al. 2011). The most common source of ignition for fires and explosions in the operating room is electrosurgical devices; nearby cloth, paper, flammable liquid, or gaseous anesthetics in an oxygen-rich environment can be ignited (Wang and Advincula 2007). A surgeon must be well-aware of the other medical devices that may be implanted in a patient prior to applying an electrosurgical technique; interference with certain types of pacemakers have the potential to lead to cardiac arrest (Gallagher, Dhinsa et al. 2011).

New tissue sealing technologies that utilize ultrasonic and bipolar technology have significantly limited the risks involved in conventional monopolar surgery. Nonetheless, electrosurgical caution and knowledge still remain a necessity for clinical safety. In addition to having an understanding of electrical basics, surgeons must be well-versed in instructions for use specific to their device and generator brand of choice. 
Informed judgment is required in order apply devices to the proper size vessels considering advanced bipolar and ultrasonic devices have the ability to seal and transect vessels up to $7 \mathrm{~mm}$ in diameter (Lyons and Law 2013). Heat production during application must also be monitored in order to minimize the risk of lateral thermal spread. Users must be aware of the audible generator tones occurring during application to indicate tissue status in addition to monitoring the effects to both the target and surrounding structures.

New technology, especially that within the generators used to power the devices, eliminates many procedure variables. For example, adaptive technology monitors the thermal state of the device blade in addition to the amount of tissue that remains in the jaws. By monitoring these factors, the tissue response generator queues a decrease in delivered power in order to prevent excess thermal injury (Broughton, Welling et al. 2013). Despite the elimination of some user-controlled variables, interfaces between technology, surgeons, and patients can still induce a heightened level of stress in the clinical setting, especially when electrical energy is involved.

\subsubsection{Medical Device Regulation and Reporting}

\subsubsection{Medical Device Regulation}

Congress granted the FDA the authority to regulate medical devices in 1976, while drugs have been mandated to prove to be safe and effective since 1938 due to the Federal Food, Drug, and Cosmetic Act (Burditt 1995). It wasn’t until morbid consequences of the Dalkon Shield intrauterine device unfolded, including reports of 209 second-trimester abortions and 11 maternal deaths, that Congress took action by enacting 
the Medical Device Amendments of 1976 (Monsein 1997). Consequences and evidence of inadequate post market surveillance in addition to lack of manufacturer adverse event reporting triggered Congress to enact the Safe Medical Devices Act of 1990 and the Medical Device Amendments of 1992 (Monsein 1997, Maisel 2004). As a result, clinical facilities were required to report serious injuries or deaths related to medical devices. Additionally, manufacturers were enforced to report device malfunctions to the FDA that cause or potentially cause patient injury (Maisel 2004).

Despite the actions by Congress, concerns still existed in regards to the FDA approval processes. The premarket approval (PMA) process was introduced by the 1976 law specifically for devices. PMA submissions require the following: "valid scientific evidence" provided by extensive testing that "provide(s) reasonable assurance that the device is safe and effective for its intended use" (Yock, Zenios et al. 2015). This rigorous process is applied rightfully to medical devices that "support or sustain human life, are of substantial importance in preventing impairment of human health, or which present a potential, unreasonable risk of illness or injury" (Maisel 2005). The 510(k) provision, an alternative regulatory standard, emerged in 1976 in addition to the PMA due to the significant number of existing devices in the market. The intention of the 510(k) provision was to provide a less burdensome and less expensive path for newer versions of existing devices to enter the market by requiring only a demonstration that the device was "substantially equivalent" in materials, purpose, and mechanism of action to another device that was already marketed.

The FDA classifies devices based on their risk profile. The lowest and intermediate risk devices are classified as Class I and Class II, respectively. Class III 
devices pose the greatest potential risk. Adopted from Biodesign: A Process of Innovating Medical Technologies, Table 1 displays a detailed outline of each device class (Yock, Zenios et al. 2015).

Table 1. FDA device classification (Yock, Zenios et al. 2015).

\begin{tabular}{|c|c|c|c|}
\hline Class & Examples & Description & FDA Regulation \\
\hline I & $\begin{array}{l}\text { Surgical gloves, } \\
\text { bandages, tongue } \\
\text { depressors }\end{array}$ & $\begin{array}{l}\text { Present minimal } \\
\text { potential harm to } \\
\text { patient and are } \\
\text { typically simple in } \\
\text { design. }\end{array}$ & $\begin{array}{l}\text { - Registration of the } \\
\text { establishment with FDA. } \\
\text { - Medical device listing. } \\
\text { - General FDA labeling } \\
\text { requirements. } \\
\text { - Compliance with quality } \\
\text { system regulation (QSR). }\end{array}$ \\
\hline II & $\begin{array}{l}\text { Suture materials, } \\
\text { powered } \\
\text { wheelchairs, x-ray } \\
\text { machines, surgical } \\
\text { needles }\end{array}$ & $\begin{array}{l}\text { Typically non-invasive } \\
\text { but more complicated } \\
\text { in design than Class I; } \\
\text { must demonstrate that } \\
\text { the devices will } \\
\text { perform as expected } \\
\text { and not cause injury or } \\
\text { harm to users. }\end{array}$ & $\begin{array}{l}\text { - Special labeling } \\
\text { requirements. } \\
\text { - Mandatory performance } \\
\text { standards. } \\
\text { - Design controls. } \\
\text { - Post-market surveillance. }\end{array}$ \\
\hline III & $\begin{array}{l}\text { Replacement heart } \\
\text { valves, silicone } \\
\text { breast implants, } \\
\text { implantable } \\
\text { pacemakers, } \\
\text { implanted } \\
\text { cerebellar } \\
\text { stimulators }\end{array}$ & $\begin{array}{l}\text { Implantable, } \\
\text { therapeutic, or life } \\
\text { sustaining high risk } \\
\text { devices. }\end{array}$ & $\begin{array}{l}\text { - Generally approved by } \\
\text { PMA regulatory pathway; } \\
\text { some eligible for } 510(\mathrm{k}) \\
\text { clearance. } \\
\text { - Valid scientific evidence } \\
\text { to demonstrate safety and } \\
\text { effectiveness before use } \\
\text { in humans. }\end{array}$ \\
\hline
\end{tabular}

Approximately $75 \%$ of Class I and less than $10 \%$ of Class II devices do not require FDA clearance to be marketed (Yock, Zenios et al. 2015). All class III devices are required to obtain FDA clearance. Of all medical devices, about half fall in Class I classification, while 45-50\% are in Class II, and 5-10\% in Class III (Yock, Zenios et al. 2015). More than 100,000 medical devices are regulated by the FDA, which are 
organized into 1,700 different categories of technology. The categories are managed by the Office of Device Evaluation (ODE), which consists of seven divisions with 33 branches based on medical specialties (CDHR 2017). A device is referred to as cleared and to have achieved premarket notification when it passes $510(\mathrm{k})$ clearance, whereas a Class III device that completes the PMA route is approved by the FDA.

The 510(k) provision was initially intended for Class I and many Class II devices that did not require significant scientific study. However, the technological evolution quickly increased device complexity, and the FDA lacked necessary resources to modify performance standards for these evolving devices or pass more devices through the rigorous PMA pathway (Zuckerman, Brown et al. 2011). Further, Congress passed the Medical Device User Free and Modernization Act of 2002 (MDUFMA) which sparked a shift in the regulatory standard. Recently, 510(k) provision has been used by the FDA as the leading mechanism for new device clearance, while only $1 \%$ of medical devices have been reviewed using the PMA process (Zuckerman, Brown et al. 2011). The Government Accountability Office (GAO) reviewed the 510(k) process and published a report in 2009 revealing that $66 \%$ of recent Class III submissions that were "implantable, life sustaining, or of significant risk" were cleared via the 510(k) process (GAO 2009). By law, all Class III devices should have been subjected to the more thorough PMA process.

The $510(\mathrm{k})$ process as applied by the FDA has been under critical observation by media, organizations, and Congress. A 2011 study by Zuckerman et al. investigated how the approval and clearance processes were used for medical devices that had been recalled for life-threatening problems (Zuckerman, Brown et al. 2011). Of 113 recalls 
between 2005-2009 examined by Zuckerman et al., 7\% were exempt from FDA regulation, $19 \%$ were approved via the PMA process, and $71 \%$ were cleared through the 510(k) process (Zuckerman, Brown et al. 2011). Further, 12\% of the recalled devices that had undergone $510(\mathrm{k})$ clearance were marketed for "risky or life-sustaining Class III indications," and, as such, should have been legally mandated for review under PMA regulations (Zuckerman, Brown et al. 2011). The study revealed differences in the approval criteria for high risk and life sustaining devices and the recall criteria that deems a device life threatening.

Issues likely stem from the FDA's efforts in balancing the approval of safe and effective devices while also promoting innovation in the medical device industry. Some criticism of the FDA is directed towards device approval processes being too quick and not rigorous enough, while other criticism is geared towards delay of the entry of important new medical devices (Challoner 2011, Yock, Zenios et al. 2015). The Institute of Medicine (IOM) committee released a report in 2011 suggesting a shift away from the $510(\mathrm{k})$ process as soon as possible and development of a new framework (Challoner 2011). Clearly regulatory process reform is necessary, and the FDA has recently taken steps indicating acknowledgement and implementation of changes intended to improve the criteria and assessments associated with the 510(k) process (Monsein 1997, Maisel 2004, Maisel 2005, Zuckerman, Brown et al. 2011, Yock, Zenios et al. 2015, CDHR 2017, FDA 2017). 


\subsubsection{Medical Device Reporting and Recalls}

Medical device reporting (MDR) is carried out by manufacturers, importers, and user facilities and is the primary means by which the FDA collects medical device adverse event reports (Yock, Zenios et al. 2015). The FDA's MAUDE database contains a significant number of medical device-related incidents and is a useful tool for identifying the nature and frequency of such incidents. Efficient searches of the database must be approached systematically as described in the study by Gupta and Pidgeon (Gupta and Pidgeon 2016). The FDA's MAUDE database reports include identifying factors such as report number, event date, report date, manufacturer, product code, and brand name. The reports typically contain an event description with details regarding the adverse event and may include a manufacturer narrative with details regarding a follow up analysis of the device involved.

Any device malfunction that causes or could cause significant injury is required to be reported to the FDA by the manufacturer. Device user facilities are required to report a suspected medical device-related death to the FDA and manufacturer. User facilities must also report serious injuries related to a medical device to the manufacturer or to the FDA if the manufacturer is unknown (FDA 2017). User facilities are not required to report a device malfunction. Healthcare professionals, consumers, and patients are encouraged to voluntarily report adverse events related to medical devices in addition to user errors, quality issues, and therapeutic failures (FDA 2017). To improve safety in the market, it has been suggested to make detailed public reporting of medical device malfunctions and device reliability mandatory; however, some physicians disagree claiming that such extensive notification is too onerous and increases physician liability 
(Maisel 2005). Mandatory and accurate reporting would supply superior information to patients and physicians enabling them to make more informed decisions regarding their choice of medical device. MDR provides a means to derive useful failure mode data directly related to surgical medical device use.

The most serious medical device recalls posted by the FDA include products with a "reasonable chance that they would cause serious health problems or death" (FDA 2017). Recalls occur due to one or both of the following: a defective medical device or when a device could put a user's health at risk. Either a correction or removal recall takes place depending on the action location. A correction recall "addresses a problem with a medical device in the place where it is used or sold," and a removal recall "addresses a problem with a medical device by removing it from where it is used or sold" (FDA 2017). Typically, a company recalls a medical device voluntarily if the product is shown to violate FDA law. If necessary, a company can be required by the FDA to recall a device. Product recalls can be accessed via the FDA Medical Device Recalls database and include information such as recall firm, center classification year, number of recalls, product name, and recall reason.

\subsubsection{Limitations of Medical Device Reporting}

Device user facilities are required to report device-related death and serious injury; however, there are no requirements as to the level of report detail submitted to the FDA. The event descriptions provided within adverse event reports may or may not provide enough information to derive a specific failure mode, which hinders a complete investigation into user and device failures. There is some general consensus among users 
in regards to the terminology used to report an adverse event, yet an overall definition and application of terms and types of failure modes still lacks. This research relied on the level of detail of adverse event reports and collected terms from the literature in order to compile the most universal terms for reporting a failure mode relative to the particular device. In regards to the FDA regulatory processes, there is no doubt that concerns from outside parties exist in terms of the acceptance criteria. While this research does not provide suggestions for regulatory reform, it does provide assistance to designers in identifying the design complexity and potential surgical device failures that could be of value to consider in the early design stages. Consequentially, such assistance helps ensure the effectiveness and safety of a device's final design in addition to the steps required for FDA approval or clearance.

\subsubsection{Surgical Device Failure Mode Studies}

\subsubsection{Endoscopic Stapler Failure Mode Studies}

Endoscopic staplers are generally reliable, yet difficulties have been noted in every step of use. Recall that endoscopic staplers simultaneously staple and cut interior tissue as discussed in Section 1.2.1.2. Problems can occur in various stages of use as identified by Deng et al. including removal from packaging, application to tissue, firing staples, cutting tissue, removal from tissue and/or port, and resulting staple line (Deng, Meng et al. 2002). Issues with packaging potentially compromise the sterility of the device or reload and may damage critical components for proper function. Improper anastomosis may result from any complications encountered during the firing sequence. 
Difficulty in releasing the device after firing can lead to various undesirable outcomes such as unintended tissue damage and loss.

Deng et al. found that abnormal firing of the stapler and improper staple formation to be the most common and fatal aspects of device error (Deng, Meng et al. 2002). A study on hemostasis in laparoscopic renal surgery indicated that endoscopic staplers had the highest risk of malfunctioning (Hassouna and Manikandan 2012). Further, it was stated that the two most common mechanisms of failure found in the investigation were inadequate staple lines and device failure to release from tissue, which result in a relatively high percentage of severe hemorrhage and open conversion during laparoscopic renal surgery (Hassouna and Manikandan 2012). These consequences heavily affect staple line integrity, which is an essential aspect of endoscopic stapling given that failure many times causes intra-operative and post-operative leaks, thus compromising a patient's recovery, health, and safety.

A study that investigated MAUDE reports of endovascular gastrointestinal anastomosis (Endo-GIA) malfunctions during laparoscopic nephrectomy indicated the most common mechanism of failure was malformed or leaking staple line after firing (Mansour, El-Nashar et al. 2014). The study analyzed 65 Endo-GIA device failures during renal vascular control in laparoscopic nephrectomy identified in the FDA MAUDE database. Malformed or a leaking staple line after firing accounted for $60 \%$ of the cases. $10.8 \%$ were attributed to device failure to release from tissue, and device handle breakage during firing occurred in 2 of the cases.

One of the most concerning complications occurs upon firing a used stapler or cartridge. There are lockout mechanisms on many endoscopic staplers that are meant to 
prevent the ability to fire a used device; however, this lockout mechanism, also referred to as a safety interlock, can be overcome with excessive force applied by an inexperienced surgeon (Deng, Meng et al. 2002). Studies have shown that preventable user error accounts for a majority of adverse event associated with endoscopic staplers (Chan, Bishoff et al. 2000, Deng, Meng et al. 2002). However, a study that surveyed 44 minimally invasive program directors indicated that the two most common malfunctions were staples partially firing and the stapler misfiring and not releasing, which are potentially device specific versus user error (Kwazneski, Six et al. 2012). The recalls identified by Brown and Woo also suggest stapler errors arise from design and manufacturing issues versus solely user error (Brown and Woo 2004).

Various failure mode types that contribute to adverse events can be difficult to classify as either user or device error. Consider events that involve malformed staples. Such an outcome could be due to firing the device on too thick of tissue; yet, this could also be an outcome of improper device function. The results of such investigations emphasize the importance of diligent and accurate reporting of adverse outcomes related to use of endoscopic stapling devices during MIS in order to determine the source of complication.

\subsubsection{Energy-Based Tissue Sealer Failure Mode Studies}

A thorough understanding of energy-based device complications is essential in order to decrease the likelihood of preventable injuries and deaths. The rate of injuries from surgical energy has been estimated as one to two per 1,000 operations (Nduka, Super et al. 1994, Overbey, Townsend et al. 2015). The rate of injury is relatively low; 
however, considering more than 50 million inpatient procedures are performed in the United States each year, continuous and additional efforts are necessary in order to reduce adverse event outcomes in the operation setting. An array of clinical hazards has been linked to electrosurgical devices overall. The most common source of ignition for fires and explosions in the operating room is electrosurgical devices; nearby cloth, paper, flammable liquid, or gaseous anesthetics in an oxygen-rich environment can be ignited (Wang and Advincula 2007). Insulation failure can create alternate pathways for current flow, and adjacent organ injury is possible with a high enough current density. Tissue burns and operation fires can also be attributed to insulation failure.

Patterns of injuries and deaths resulting from energy-based devices in general have been identified as thermal burn, hemorrhage, mechanical failure, and fire. Injury mechanisms of failure modes include inadvertent application, dispersive electrode failure, capacitive coupling, residual heat, insulation failure, and direct application.

Electrosurgical failures are typically associated with monopolar devices and earlier bipolar devices. Many of these complications have been mitigated by the implementation of advanced technologies, yet adverse outcomes can still occur. In a study by Overbey et al., ultrasonic energy accounted for 19\% of the mechanical failures. Advanced bipolar devices accounted for $48 \%$ of broken devices indicated in the analysis, and $37 \%$ of the retained object reports were associated with ultrasonic energy (Overbey, Townsend et al. 2015). Residual heat injury has been most commonly associated with ultrasonic devices, in which injury occurs from a heated electrode after completion of the activation cycle; $21 \%$ of thermal burns due to residual heat were attributed to ultrasonic energy (Overbey, Townsend et al. 2015). Ultrasonic and advanced bipolar devices were most commonly 
associated with bleeding events, which is likely due to the nature of their specific function in a surgical procedure (Overbey, Townsend et al. 2015). Another disadvantage and potential hazard of ultrasonic energy is the formation of aerosolized fatty droplets during activation on tissue, which can interfere with endoscopic visualization (Alkatout, Schollmeyer et al. 2012). Also, despite lower operating temperatures, ultrasonic energy has been correlated with substantial and undesirable tissue heating (Person, Vivas et al. 2008).

\subsubsection{Limitations of Failure Mode Studies}

Studies have described failure modes of endoscopic staplers and energy-based devices via query of the FDA MAUDE database. However, these studies' assessments of failure modes are predominantly approached from a clinical standpoint versus a design or engineering perspective. The few studies that mention device or component failure are vague and do not discuss the particular mechanism of failure that would assist designers and engineers in developing new devices or implementing modifications for design improvement. The manufacturers of surgical devices conduct extensive tests and research related to device failure modes; however, this information is not made accessible by the general public or device users. Published information related specifically to device design complexity in relation to failure mode outcomes can aid the decisions of clinical facilities, users, and, in some cases, patients in regards to procedural approaches that involve such surgical devices.

The studies aimed at investigation into the FDA MAUDE database typically include a very broad range of device types and technologies. While that approach 
provides very useful general information for users, it does not allow for an in-depth discussion of specific device-related failures. This research investigated the specific user and device failure modes derived from adverse event reports directly associated with endoscopic staplers and energy-based tissue sealers.

Any mechanical device, such as the surgical medical devices discussed herein, possesses the potential to malfunction (Deng, Meng et al. 2002). Device failure at any stage of use during MIS has the potential to cause severe hemorrhage and conversion to open surgery. A comprehensive understanding of device failure is crucial in order to identify user error and device-related failures. Such an understanding helps to reduce and eliminate user and device error and, therefore, improves the safety, efficiency, and usability of a device (Gupta and Pidgeon 2016). The rate of adverse events reported regarding endoscopic stapler and energy-based device malfunction has been shown to be relatively low when compared to their overall frequency of use (Chan, Bishoff et al. 2000, Deng, Meng et al. 2002, Brown and Woo 2004, Overbey, Townsend et al. 2015). However, thorough and on-going investigation is paramount due to potential catastrophic consequences of device failure.

\subsubsection{Severity Grading Methods}

\subsubsection{Overview of Current Methods}

Classification and severity grading of adverse event outcomes is necessary for a common assessment and evaluation of procedural concerns that are both user and surgical instrument related. Various methods for grading surgical complications have been developed, such as the T92 and its derivatives. Strasberg et al. proposed a modification 
to the grading system, known as the Accordion Classification system, used to determine the severity of postoperative complications (Strasberg, Linehan et al. 2009). The Accordion Classification shows promise towards a common severity grading system for surgical complications by providing a more understandable and accessible method, yet further modifications are still required to cover an even wider range of procedure incidents. The Abbreviated Injury Scale (AIS) ranks the severity of injury as an anatomical scoring system (Copes, Champion et al. 1990). The scoring system ranks on a scale of 1 to 6 . The Injury Severity Score (ISS) and the New Injury Severity Score (NISS) focus on injury associated with a particular region of the body, such as head, face, chest, etc. (Baker, O'Neill et al. 1974). Other injury severity scoring methods exist, some of which are applied specifically to intraoperative surgical complications (Bruce, Russell et al. 2001, Dindo, Demartines et al. 2004, Dekutoski, Norvell et al. 2010, Porembka, Hall et al. 2010).

The International Classification of Diseases (ICD) aims to identify, classify, and document mortality statistics. Reported causes of death are translated into medical codes using the latest revision of the ICD, which to date would be the ICD-10 (CDC 2016). The ICD codes have been utilized for a variety of other purposes within the medical field, including the characterization of injury severity and the likelihood of death and medically-caused injuries. Samore et al. compared detections methods for medical device-related hazards and adverse events in hospitalized patients (Samore, Evans et al. 2004). The use of ICD-9 codes was useful in the surveillance of adverse events due to the consistent application across various institutions, yet it was not adequate to serve as a surveillance standard (Samore, Evans et al. 2004). While the ICD-9 codes appeared 
beneficial for surveillance of device-related adverse events that resulted in hospitalization, it was not as useful for the adverse events that took place during hospitalization.

The Government of New South Wales (NSW) in Australia utilizes Severity Assessment Codes (SACs) in the NSW Health Incident Management Process (NSW 2014, Mitchell, Williamson et al. 2015). This process outlines the required steps for each level of SAC for application to adverse clinical incidents in public hospitals. The clinical incidents are defined as "any unplanned event which causes, or has the potential to cause, harm to a patient" and are reported to an Incident Information Management System (IIMS) (NSW 2014). Clinical incidents are classified SACs, with SAC1 being the most serious and SAC4 being the least severe.

As defined by the National Cancer Institute (NCI), an adverse event is "any unfavorable and unintended sign (including an abnormal laboratory finding), symptom, or disease temporarily associated with the use of a medical treatment or procedure that may or may not be considered related to the medical treatment or procedure" (NIH 2003). NCI developed the Common Toxicity Criteria (CTC) system in 1983. The CTC system went through various revisions, and the most recent version was developed in order to move away from the term "toxicity." The Common Terminology Criteria for Adverse Events v3.0 (CTCAE v3.0) emerged to fulfill this purpose and, upon release in 2003, represented the "first comprehensive, multimodality grading system to include both acute and later effects" (Trotti, Colevas et al. 2003). Implementation of such a system assists adverse event report standardization, comparison, and completion. The CTCAE v3.0 is organized by organ system categories and incorporates a 1-5 point grading scale (NIH 
2003). Grading refers to the severity of the adverse event, and the CTCAE v3.0 includes unique clinical descriptions for each severity.

Grading is specific to the adverse event depending on the category. Applicable to this research are the following adverse event categories listed in the CTCAE v3.0: intraoperative injury and hemorrhage/bleeding associate with surgery, intraoperative or postoperative. The grading criteria for these categories are shown in Table 2.

Table 2. CTCAE v3.0 category-specific grading (NIH 2003).

\begin{tabular}{|c|c|c|c|c|c|}
\hline $\begin{array}{c}\text { Adverse } \\
\text { Event }\end{array}$ & Grade 1 & Grade 2 & Grade 3 & Grade 4 & Grade 5 \\
\hline $\begin{array}{l}\text { Intra- } \\
\text { operative } \\
\text { injury }\end{array}$ & $\begin{array}{l}\text { Primary } \\
\text { repair of } \\
\text { injured } \\
\text { organ/ } \\
\text { structure } \\
\text { indicated }\end{array}$ & $\begin{array}{l}\text { Partial } \\
\text { resection of } \\
\text { injured } \\
\text { organ/ } \\
\text { structure } \\
\text { indicated }\end{array}$ & $\begin{array}{l}\text { Complete } \\
\text { resection or } \\
\text { reconstruction of } \\
\text { injured organ/ } \\
\text { structure indicated }\end{array}$ & $\begin{array}{l}\text { Life } \\
\text { threatening } \\
\text { consequences; } \\
\text { disabling }\end{array}$ & - \\
\hline $\begin{array}{l}\text { Hemorrhage/ } \\
\text { bleeding } \\
\text { associated } \\
\text { with surgery, } \\
\text { intra- } \\
\text { operative or } \\
\text { postoperative }\end{array}$ & - & - & $\begin{array}{l}\text { Requiring } \\
\text { transfusion of } 2 \\
\text { units non- } \\
\text { autologous } \\
\text { (10cc/kg for } \\
\text { pediatrics) pRBCs } \\
\text { beyond protocol } \\
\text { specification; } \\
\text { postoperative } \\
\text { interventional } \\
\text { radiology, } \\
\text { endoscopic or } \\
\text { operative } \\
\text { intervention } \\
\text { indicated }\end{array}$ & $\begin{array}{l}\text { Life } \\
\text { threatening } \\
\text { consequences }\end{array}$ & Death \\
\hline
\end{tabular}

A fourth version of the CTCAE was published in 2009 which modified various categories and grades that were provided in the third version. The expansion of the grading descriptions is shown in Table 3. Instrumental activities of daily living (ADL) 
refer to preparing meals, shopping for groceries or clothes, using the telephone, managing money, etc. (NIH 2009). Self-care ADL refer to bathing, dressing and undressing, feeding self, using the toilet, taking medication, and not bedridden. The CTCAE versions have been commonly used in clinical trials to document toxic effects caused by cancer treatments (Liu, Zhu et al. 2012).

Table 3. CTCAE v4.0 grading (NIH 2009).

\begin{tabular}{|c|l|}
\hline Grade & \multicolumn{1}{c|}{ Description } \\
\hline 1 & $\begin{array}{l}\text { Mild; asymptomatic or mild symptoms; clinical or diagnostic } \\
\text { observations only; intervention not indicated. }\end{array}$ \\
\hline 2 & $\begin{array}{l}\text { Moderate; minimal, local or noninvasive intervention indicated; } \\
\text { limiting age-appropriate instrumental ADL. }\end{array}$ \\
\hline 3 & $\begin{array}{l}\text { Severe or medically significant but not immediately life-threatening; } \\
\text { hospitalization or prolongation of hospitalization indicated; disabling; } \\
\text { limiting self-care ADL. }\end{array}$ \\
\hline 4 & Life-threatening consequences; urgent intervention indicated. \\
\hline 5 & Death related to AE. \\
\hline
\end{tabular}

\subsubsection{Limitations of Severity Grading Methods}

Methods exist for both surveillance and severity grading of adverse events, yet many are not sufficient for the objectives of this research. The Accordion Classification Grading System was developed for surgical complications encountered postoperatively versus intraoperative. While the use of classification could be applicable to intraoperative complications, this may present threshold difficulties due to the range of possible events and variables in such situations (Strasberg, Linehan et al. 2009). The ICD codes work well for the surveillance of medical device-related adverse events, particularly for those events that result in patient hospitalization. However, it has been noted to be less useful for adverse events encountered during hospitalization, which is the 
primary concern for investigating procedural adverse events (Samore, Evans et al. 2004). Further, the ICD surveillance system only allows broad classifications of adverse events by device type, whereas more specific classifications are desirable (Samore, Evans et al. 2004). The CTCAE grading method was deemed the most appropriate grading method for intraoperative and post-operative surgical complications associated with adverse events provided the descriptions listed for each grade. Specifically, the CTCAE v4.0 grading descriptions can be applied in order to assess the severity of adverse event outcomes associated with intraoperative minimally invasive surgical device use and postoperative outcomes.

\subsubsection{Functional Modeling}

The roots of flow-based functional modeling can be traced back to the field of Value Analysis (Rodenacker 1971, Miles 1972). From these early representations of functions in Value Analysis, researchers have continued work to effectively and accurately describe functionality (Roth 1981, Koller 1985, Hundal 1990, Little, Wood et al. 1997, Szykman, Racz et al. 1999, Stone and Wood 2000, Hirtz, Stone et al. 2002, Pahl, Beitz et al. 2007). In engineering design, a functional model is often a description of a product in terms of the elementary functions and flows that are required to achieve the product's overall function or purpose. A graphical form of a functional model is represented by a collection of sub-functions connected by the flows on which they

operate (Kurfman, Stone et al. 2000). The structure is a way for a designer to see what 
type of functions are performed without being distracted by any particular form the system and components may take.

Flow-based functional models stemming from the Pahl and Beitz methodology are perhaps the more common forms of functional models in engineering design (Pahl and Beitz 2013). Models are generated at two levels of abstraction: a black box model and a sub-functional model. Black box functional models are stand-alone functional models abstracting a high-level transformation intended for the product to complete and are generated based on the system design requirements. A functional model decomposes the overall functional black box into specific flow transformations. Flow transformations define the operations required of the system such that the identified input flows become the identified output flows through the operation of the system. Material flows are bold arrows; energy flows are thin arrows; and signals are dashed arrows.

Stone et al. developed the general framework for functional modeling (Stone and Wood 2000) and Nagel et al. developed an algorithmic approach to teaching functionality (Nagel, Bohm et al. 2012). The Nagel et al. approach uses a series of grammar rules to assemble function chains from a list of enumerated functions desired of the final product. Function chains are then aggregated into a complete functional model which represents a system or product. Creating a functional model consists of three primary steps; black box model, chains, and the aggregated functional model. Nagel et al. provide an example of a 
black box model, chains, and an aggregated functional model shown in Figures 1, 2, and 3, respectively (Nagel, Bohm et al. 2015).

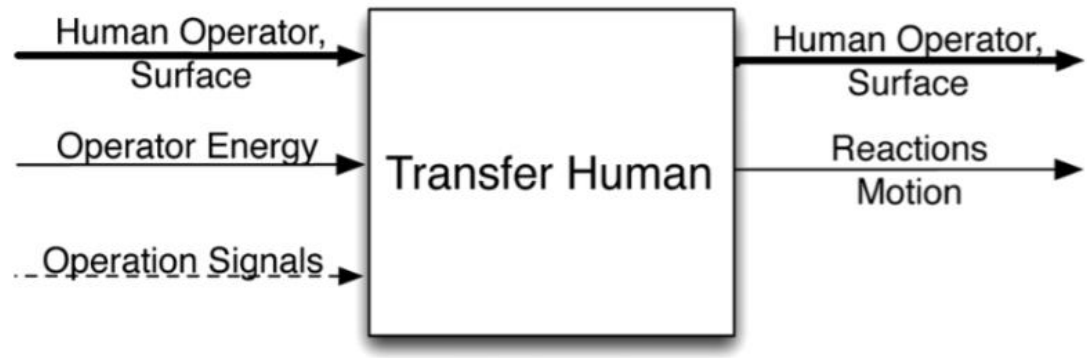

Figure 1. Black box model (Nagel, Bohm et al. 2015).

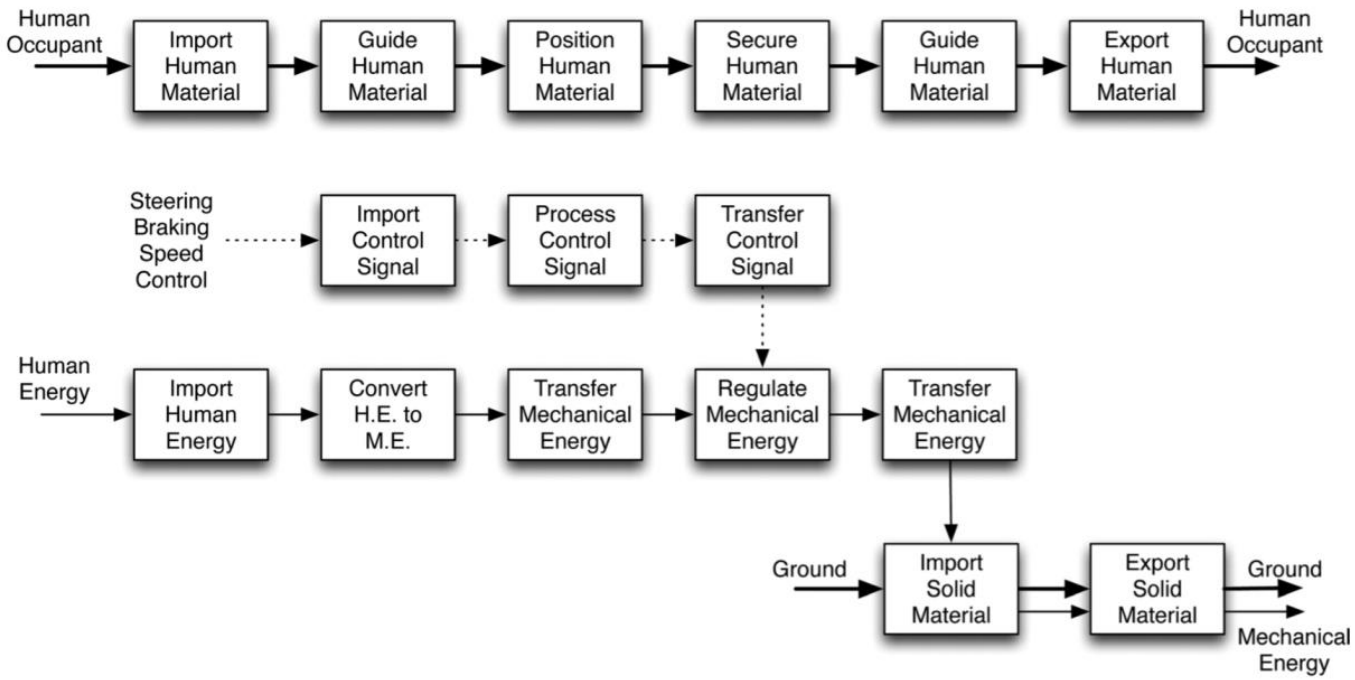

Figure 2. Functional modeling chains (Nagel, Bohm et al. 2015). 


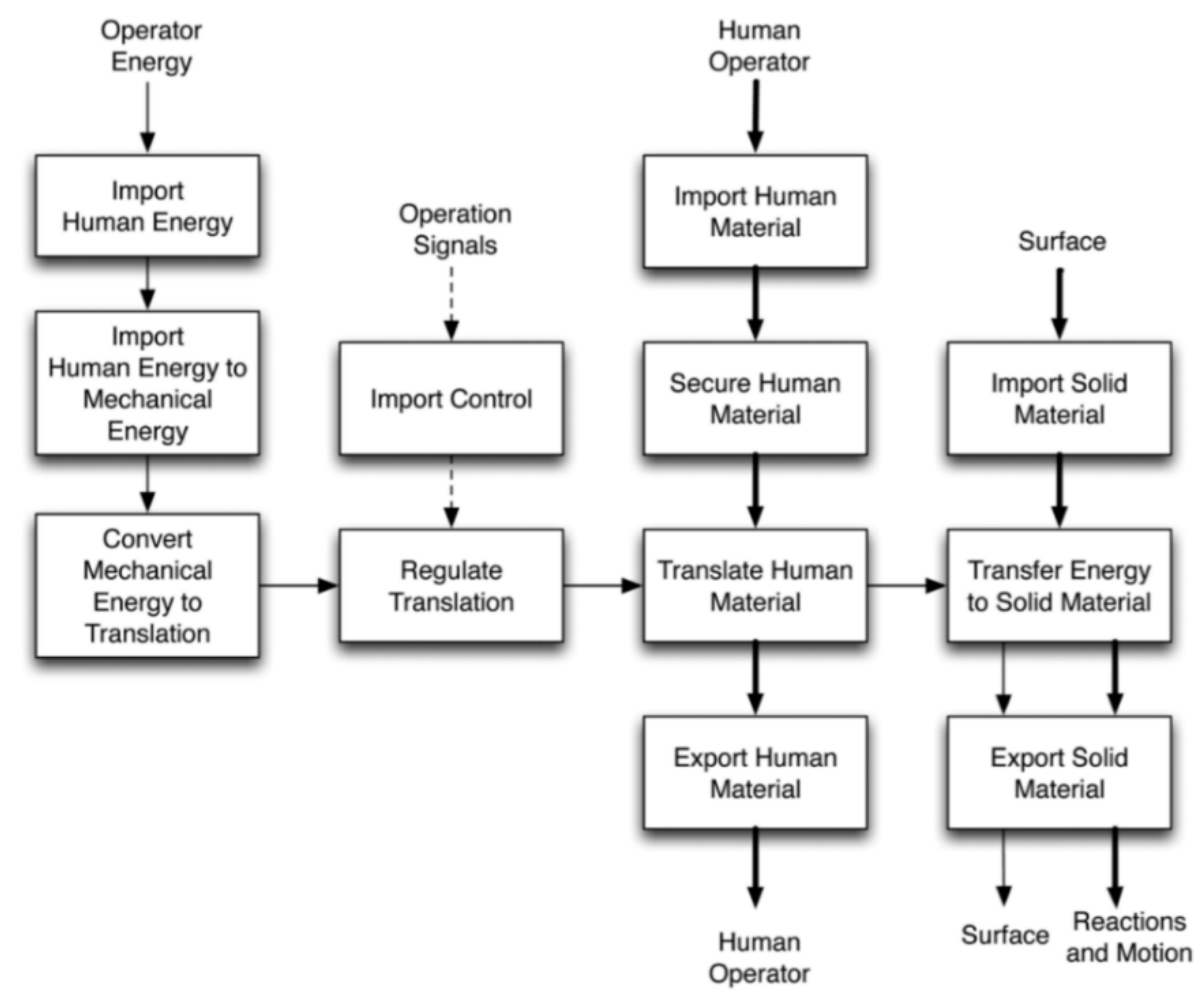

Figure 3. Functional model (Nagel, Bohm et al. 2015).

\subsubsection{Design Complexity}

\subsubsection{Surgical Device Design Complexity}

The design of surgical devices has evolved to include more than the basic mechanical mechanisms for creating a proper anastomosis. Designing devices for surgical procedures presents many challenges and must address efficiency, accuracy, and ease of use. Modularity should also be considered for surgical devices in particular due to the flexibility provided for product variations and technology development without requiring overall design changes (Hölttä and Otto 2005). The evolution of both technology and user demands potentially affects the complexity of medical devices. Consideration of an objective measure of complexity aids systematic reduction of 
unnecessary design elements and enables quantitative comparisons of design solutions (Ameri, Summers et al. 2008).

Mitchell et al. conducted a study to examine non-fatal adverse clinical incidents through use of a human factors classification framework (Mitchell, Williamson et al. 2015). Casual factors for medication and medical device related adverse clinical incidents were identified. Medical equipment breakage/failure was found to be the most common precursor event for medical device related incidents (46.0\%), and $22.5 \%$ of medical device related incidents occurred intraoperatively (Mitchell, Williamson et al. 2015). Further, Mitchell et al. suggested that medical device failure or breakage can be an indication of design flaws, inadequate maintenance of devices, and lack of device examination before use (Mitchell, Williamson et al. 2015). While the study assessed human factors related to a wide range of clinical incidents involving both medication and medical devices, it demonstrated the need to address design related aspects of medical devices. Complexity of a device's function and design is a factor that should be considered when addressing both user and device based failures and adverse event outcomes.

Complexity of a surgical device's design has the potential to inhibit a user's understanding of proper use and techniques for preventing failure; it could also impact proper device functionality. Identifying specific ways by which the differences in design complexity of surgical devices influence the occurrence and severity of failure modes is necessary in order to prevent further complication by contributing to user and designer knowledge. Assessment of these differences can be achieved through an investigation of the most utilized device brands within surgical device classes. Quantifying complexity 
allows the determination of optimal complexity levels, which, in turn, can maximize product performance. Understanding the complexity of surgical devices potentially provides valuable insight into specific mechanisms of failure and a means to implement countermeasures against undesired outcomes in the surgical setting.

Complexity is defined in many ways, at different levels of abstraction, and at different stages of design (Braha and Maimon 1998, Maier and Rechtin 2000, Blackenfelt 2001, Hölttä and Otto 2005, Summers and Shah 2010, Jacobs 2013), which render it highly contextual and subjective. The issue of generality is frequently exhibited in literature when considering complexity because each methodology defines complexity to exist only within its realm of investigation. Complexity may be necessary for product success in certain cases, though, traditionally is viewed as unfavorable. It can also be viewed as a hindrance if unnecessary functions or attributes are added to a product. Such unnecessary functions or attributes could lead to more involved design efforts, greater manufacturing or assembly work, and higher production costs. Developing product requirements or customer needs is an effective way to explicate significant product functions and mitigate useless ones.

Various complexity assessment methods have been developed for one or more of the following: design problem, design process, and design product. In terms of the design product, complexity can be studied from structural, functional, and behavioral perspectives. The measurement of information applies to the structural complexity, which is a function of the information content as represented, whereas the probability of successfully satisfying all design requirements applies to the functional complexity 
(Ameri, Summers et al. 2008). Understanding the functional complexity of a product can be beneficial in the early stages of design as an indicator of post design complexity.

The ability to identify complex systems, sub-systems, and components allows design teams to consider less complex alternatives earlier in the design process. As designers and managers seek to mitigate complexity, having early indicators are paramount to keeping project costs low. Quantifying complexity allows the determination of optimal complexity levels, which, in turn, can maximize product performance. When considering complexity in a product development domain, it is generally considered to have an adverse effect on product performance, quality, and manufacturability (ElMaraghy, ElMaraghy et al. 2012). However, it is unclear exactly what complexity is and how it can be measured on a general scale.

\subsubsection{Generalized Complexity Index}

Considering complexity is related to a product's design variables, the Generalized Complexity Index (GCI) developed by Jacobs is a method suggested to be applicable to design products (Jacobs 2013). The GCI requires scrutiny of three factors; 1.) multiplicity, 2.) diversity, and 3.) interconnectedness. Multiplicity is defined as the number of variants or versions of a product or the number of suppliers if evaluating at the supply chain level (Closs, Jacobs et al. 2008, Bozarth, Warsing et al. 2009, Closs, Nyaga et al. 2010). Diversity refers to the degree of dissimilarity seen across the elements and can be quantified by comparing the number of unique elements to the total number of elements within a system. Interconnectedness is a ratio of the number of connections within a system and the total number of possible connections. The degree of 
interconnectedness can be illustrated and derived through the use of a Design Structure Matrix (DSM) (Otto 2001, Otto and Wood 2001, Hommes and Berry 2003, English and Bloebaum 2008, Pahl and Beitz 2013). For the GCI, Jacobs prescribes a simple mathematical formula to calculate complexity, and Table 4 provides an explanation of the variables. The GCI is calculated using:

$$
\mathrm{GCI}=\mathrm{V}\left(1-\frac{\mathrm{U}}{\mathrm{T}}\right)\left(\frac{\mathrm{A}}{\mathrm{M}}\right)
$$

Table 4. Description of variables in the generalized complexity index.

\begin{tabular}{|c|l|}
\hline Variable & \multicolumn{1}{|c|}{ Description } \\
\hline V & Number of Variants \\
\hline U & Number of unique elements \\
\hline T & Total number of elements \\
\hline A & Number of connections \\
\hline M & $\begin{array}{l}\text { Maximum number of } \\
\text { connections }\end{array}$ \\
\hline
\end{tabular}

A DSM is a compact and visual representation of a system, project, or artifact in the form of a square matrix (Eppinger and Browning 2012). DSMs have been used in aerospace, manufacturing, and software engineering industries as well as research and academia (Makins and Miller 2000, Ahmadi, Roemer et al. 2001, Sullivan, Griswold et al. 2001, Guenov and Barker 2005, Farid and McFarlane 2006, Lambe and Martins 2012). Example DSM applications are estimation of product development time, definition of complex system interactions, and determining system modularity (Carrascosa, Eppinger et al. 1998, Sullivan, Griswold et al. 2001, Eppinger and Browning 2012). DSMs are widely used because of their ease of readability even when mapping 
becomes complex. DSMs are constructed by listing system elements in a square matrix then noting the interactions between elements with a value. Whole numbers, dots, or even probabilities are utilized to signify element interactions. Previous investigation reveals that while the GCI may be suited for application to supply chains, it does not accurately represent the complexity of a product (Mountain, Bohm et al. 2016, Riggs, Bohm et al. 2016).

\subsubsection{Connectivity Algorithm}

Ameri et al. investigated methods and measures of engineering design complexity (Ameri, Summers et al. 2008). In their research, the common aspects required for complexity measures in design were identified as size, coupling between two elements, and solvability. Size refers to numerous elemental counts including functional requirements, constraints applied, number of design variables, etc. Complexity as coupling is represented as the degree of decomposability of the bipartite entityrelationship graph corresponding to the representation of interest, whether design problem, product, or process (Ameri, Summers et al. 2008, Summers and Shah 2010). The solvability refers to whether the design product may be predicted to satisfy the design problem. Ameri et al. proposed and validated measures via a set of experiments; size and coupling complexities of consumer products were studied based on a function structure, connectivity graph, and parametric associativity graph (Ameri, Summers et al. 2008). Sizing and coupling were concluded to be independent entities and coupling complexity is less dependent on representation than size complexity (Ameri, Summers et al. 2008). 
Coupling complexity accounts for a measure derived from the connections between variables at multiple levels represented in a graph-based format (Summers and Shah 2010). A connectivity algorithm has been proposed in the literature to measure the connectivity of an entity-relationship graph via removal of relationships between entities until the graph is decomposed into individual entities (Ameri, Summers et al. 2008). Graphs are repeatedly separated into sub-graphs until single nodes remain. Adopted from a study by Ameri et al., Table 5 outlines the connectivity complexity algorithm (Ameri, Summers et al. 2008). Once the algorithm has been applied, a decomposability score results, which measures how difficult it is to take the product or system apart piece by piece. Complexity increases with the more steps, relationship sets, or relationships per separated element that are required to decompose the product or system entirely.

Table 5. Connectivity algorithm (Ameri, Summers et al. 2008).

\begin{tabular}{|l|}
\hline \multicolumn{1}{|c|}{ Connectivity Algorithm } \\
\hline 1. Eliminate Unary Relations \\
\hline 2. Initialize values: level $=1$; total $=0$; \\
\hline 3. For each graph to be searched \\
a) Initialize set size $=1$ \\
b) For all combinations of relations in a set size \\
i. Remove set size relations from graph \\
ii. Check for separation \\
iii. If separated graphs, mark the relation set removed \\
c) If no relation set removed, increment set size and return to 3b \\
d) For all relations sets marked, find the combination of sets that remove the \\
most relations without duplicate removal (number of sets) \\
e) Calculate score: level * set level * number of sets + total \\
f) Submit each distinct graph to 3 \\
\hline
\end{tabular}

The connectivity algorithm may be appropriately applied using the following graph-based formats: bipartite product representations derived from function structures, 
connectivity graphs, parametric associativity graphs, or DSMs. The level of interconnectedness of functions in a product is revealed within the function structure, whereas the existence and type of component connections within a product are well represented by connectivity graphs (Summers and Shah 2010). A bipartite graph should be used first for the algorithm to represent a product considering that fundamentally the coupling complexity is a measure of the level of a product's decomposability (Mohinder, Gill et al. 2016). Conversely, a parametric associativity graph narrows in on the manner in which components are connected together through their parameters, for example, component material and geometric dimensions. Utilizing the algorithm for various representations provides different views, indicating the importance of consistent graph formats during product complexity comparisons.

\section{$\underline{1.3 \text { Summary }}$}

While there are numerous studies aimed at improving surgeons understanding of surgical devices via implementing education tools (McColl, Karmali et al. 2009, Feldman, Brunt et al. 2013, Madani, Watanabe et al. 2014), little to no research has been carried out that assists engineers in early identification of specific design variables that are potentially detrimental to the device's or user's performance in the surgical setting. A multidisciplinary approach to MIS is required in order to implement improved systems, methods, tools, and instruction in order to mitigate errors and prevent patient harm (Cuschieri 2006). The specific aims carried out in support of the overall research goal provide users and manufacturers with insight regarding the phases of operation and 
mechanisms responsible for the failures related to surgical devices. The outcomes of reports retrieved from the FDA MAUDE database were examined in conjunction with a complexity assessment for specific classes of surgical devices to investigate the effect of a device complexity assessment on the severity of adverse event outcomes. A universally accepted complexity score has not yet been developed; however, by applying the existing connectivity algorithm to surgical devices, a comparative complexity measure can be determined and utilized.

Investigation of minimally invasive surgical device failure modes via query of the FDA's MAUDE has been deemed valuable to trainee and experienced physicians (Deng, Meng et al. 2002, Brown and Woo 2004, Fuller, Ashar et al. 2005, Mansour, El-Nashar et al. 2014, Overbey, Townsend et al. 2015), while a complexity assessment aids designers in objective and quantifiable comparisons of design optimization, alternative design solutions, and cost estimation (Ameri, Summers et al. 2008). Given the existing evidence showing that many surgeons are not aware of the tissue handling characteristics and limitations of new or reengineered devices is reason for concern; such a knowledge gap can compromise patient safety (Mery, Shafi et al. 2008, Feldman, Fuchshuber et al. 2012, Feldman, Brunt et al. 2013, Chekan and Whelan 2014, Overbey, Townsend et al. 2015, Madani, Watanabe et al. 2016, Madani, Watanabe et al. 2016). By investigating devices that utilize available and advancing technologies from various manufacturers, this research examined a broad range of operationally diverse classes of surgical devices. As a result, the approach presented is applicable to an array of surgical practices and contributes knowledge and awareness to a widespread audience that encompasses users and designers. Thorough and on-going investigation into device complexity and adverse 
event outcomes is essential to ensuring patient safety and preventing serious injury and death. The research contributions herein supplement efforts to bridge the gap between surgeons and engineers to ensure the successful implementation and evaluation of new and modified surgical device technology in the operating room. 


\section{CHAPTER II \\ SURGICAL DEVICE FAILURE MODES}

\section{$\underline{\text { 2.1 Failure Mode Study Overview }}$}

The use of endoscopic staplers and energy-based tissue sealers is increasing along with their application to more wide-ranging procedures due to design and procedural evolution. Enhanced features and safety of surgical devices encourages such increases in use and application; therefore, it is necessary to characterize the nature and frequency of the failures associated with these devices so as to better understand the mechanisms of failure. The approach presented contributes to user and designer knowledge allowing for potential improvements as devices and surgical procedures advance. This chapter addresses Specific Aim 1, which was to describe the types of failure modes shown to be associated with endoscopic staplers and energy-based tissue sealers used in MIS based on reported adverse events.

The FDA MAUDE database was queried for product codes and terms specific to the devices of interest. A random sample of reports was utilized in determining the types and frequencies of failure modes associated with each device. The failure modes were grouped by phase of device operation. The literature reveals that user error is said to account for the majority of failure as discussed in Section 1.2.4; however, device-based 
failures are also prevalent and important to identify (Deng, Meng et al. 2002). Therefore, recalls associated with each device were also retrieved as another means to identify the presence of device related issues. The data reported reveals prevalence of both user and device-based errors, which further supports an investigation into adverse events from both a clinical and design perspective.

\section{$\underline{2.2 \text { Failure Mode Study Methods }}$}

\subsubsection{FDA Database Query}

The FDA MAUDE database classifies reports using the following event types: malfunction, injury, and death. The FDA defines an adverse event as "any undesirable experience associated with the use of a medical product in a patient" (FDA 2016). An adverse event has also been defined as "any unfavorable and unintended sign (including abnormal laboratory finding), symptom, or disease temporally associated with the use of a medical treatment or procedure that may or may not be considered related to the medical treatment or procedure". These definitions were utilized, and an adverse event was considered to be an undesirable and unintended outcome as the result of a procedure involving a surgical device that specifically resulted in an injury or morbid outcome. Therefore, the events categorized as a death and injury by the reporting facility in the FDA database were retrieved for analysis while reports involving a malfunction event type were excluded. 


\subsubsection{Endoscopic Stapler FDA Database Query}

Reports involving endoscopic staplers were retrieved from the FDA MAUDE database between January 1, 2006 and January 1, 2016 under the product codes listed in Table 6.

Table 6. FDA product code search for endoscopic staplers.

\begin{tabular}{|c|c|}
\hline Product Code & Device \\
\hline GAG & Stapler, surgical \\
\hline GDW & Staple, implantable \\
\hline
\end{tabular}

The inquiry was optimized by searching for the following manufacturer and brand names of interest: ETS manufactured by Ethicon Endo-Surgery Inc. and the Endo GIA manufactured by Covidien Ltd. Ethicon is a subsidiary of Johnson \& Johnson. The specific device of interest manufactured by Ethicon is classified as an Endocutter, which is a class of products in the surgery franchise that attributed to its market growth in 2015 (JnJ 2016). Due to the various transfers of ownership of the Endo GIA stapler, the device was also listed under US Surgical, Tyco Healthcare, and AutoSuture; therefore only the brand name Endo GIA was utilized for report retrieval. The reports were downloaded from the FDA website and filtered in Excel for relevant terms that indicated an adverse event associated with the endoscopic staplers of interest.

\subsubsection{Energy-Based Tissue Sealer FDA Database Query}

The FDA MAUDE database was queried for the target energy-based devices which are identified as laparoscopic tissue sealers that utilize either ultrasonic 
technology, bipolar technology, or both. The product codes to be included in the search are shown in Table 7.

Table 7. FDA product code search for bipolar and ultrasonic devices.

\begin{tabular}{|c|c|}
\hline Product Code & Device \\
\hline GEI & $\begin{array}{c}\text { Electrosurgical, cutting \& } \\
\text { coagulation \& accessories }\end{array}$ \\
\hline LFL & Instrument, ultrasonic surgical \\
\hline
\end{tabular}

The choice of product codes utilized in this research was driven by a query of the MAUDE for electrosurgical cutting and coagulation devices and accessories by Overbey et al. (Overbey, Townsend et al. 2015). Their query revealed that $87 \%$ of the reports were classified under GEI, 10\% were reported under LFL, and 3\% were reported under the remaining product codes in the category. The keywords used for the search under these product codes include the most utilized vessel sealing technologies in the field (Lyons and Law 2013). The companies involved in the manufacture of such devices includes, but is not limited to, Covidien Ltd. (Medtronic), Ethicon Endo-Surgery, and Olympus. Covidien manufactures a vessel sealer and divider known under the brand LigaSure $^{\mathrm{TM}}$ and an ultrasonic device under the brand Sonicision. Ethicon manufactures both an advanced bipolar tissue sealer and an ultrasonic device branded under the names Enseal® and Harmonic Ace ${ }^{\circledR}$, respectively. Olympus currently supplies the Thunderbeat, the world's first and only full-integrated bipolar and ultrasonic technology for tissue management to date (Olympus 2012, Obonna and Mishra 2014).

The energy-based tissue sealers retrieved for this analysis were the Thunderbeat manufactured by Olympus and the Harmonic Ace manufactured by Ethicon Endo- 
Surgery, Inc. Reports for the Ethicon Harmonic Ace were retrieved from the FDA's MAUDE database between January 1, 2006 and January 1, 2016. Due to the relatively recent release date of the Olympus Thunderbeat, the entire sample of reports involving death and injury related to the Thunderbeat was retrieved up to what was available in the database on April 19 $9^{\text {th }}, 2017$.

\subsubsection{Failure Mode Study Sample}

Table 8 shows the number of adverse event reports (death and injury) retrieved from the FDA database for each device of interest.

Table 8. Number of death and injury reports downloaded from MAUDE database.

\begin{tabular}{|c|c|c|}
\hline Device & No. Death Reports & No. Injury Reports \\
\hline Ethicon ETS & 32 & 647 \\
\hline Covidien Endo GIA & 38 & 2039 \\
\hline Ethicon Harmonic Ace & 10 & 280 \\
\hline Olympus Thunderbeat & 8 & 77 \\
\hline
\end{tabular}

Reports with event descriptions that did not provide sufficient explanation of the adverse event were excluded from the random sample analysis. In addition, irrelevant device reports, duplicate reports, and reports with unspecified event types were excluded from the overall analysis. Sample size was chosen according to the rule-of-thumb for regression and correlations and verified using GPower (Green 1991, Voorhis and Morgan 2007, Austin and Steyerberg 2015). All reports were selected at random, and a proportionate number of death and injury reports relative to the total number were selected to attain 100 cases for each device. The reports were sorted from smallest to 
largest based on a random number generated for each report using Excel. The sample included the total population of Thunderbeat reports and 100 reports associated with each of the remaining devices. The final sample is shown in Table 9.

Table 9. Number of death and injury reports included in the sample for analysis.

\begin{tabular}{|c|c|c|c|}
\hline Device & $\begin{array}{c}\text { No. Death } \\
\text { Reports }\end{array}$ & $\begin{array}{c}\text { No. Injury } \\
\text { Reports }\end{array}$ & Total Sample \\
\hline Ethicon ETS & 5 & 95 & 100 \\
\hline Covidien Endo GIA & 2 & 98 & 100 \\
\hline Ethicon Harmonic Ace & 3 & 97 & 100 \\
\hline Olympus Thunderbeat & 8 & 75 & 83 \\
\hline
\end{tabular}

\subsubsection{Failure Mode Study Data Analysis}

\subsubsection{Failure Mode Data Analysis}

Failure modes and the phase in which failure occurred were identified for each adverse event report. Endoscopic stapler user and anatomical failure modes have been identified in the literature (Deng, Meng et al. 2002, Brown and Woo 2004, Mery, Shafi et al. 2008, Robert, Poncet et al. 2011, Kwazneski, Six et al. 2012) and has been expanded upon through analysis of the adverse event reports retrieved (Riggs, Bohm et al. 2016). The user and device-based failure mode taxonomy associated with laparoscopic vessel sealing technology from the literature review was applied (Tucker and Voyles 1995, Hay 2005, Massarweh, Cosgriff et al. 2006, Wang and Advincula 2007, Gallagher, Dhinsa et al. , Alkatout, Schollmeyer et al. 2012, Lyons and Law 2013, Overbey, Townsend et al. 2015). It should be noted that in some cases when a failure mode was not explicitly stated, the failure mode was inferred based on the context provided in the event 
description and manufacturer narrative. Manufacturer narrative excerpts were included in the results to denote the type of content and inferences that can be acquired.

The majority of anatomical and user errors were derived from the reporting facility's narrative while the mechanical and electrical device-based failures were typically stated more explicitly in the manufacturer narrative of the event description within the reports. In general, the number of reporting facility and user narratives outweigh the appearances of manufacturer narratives (Riggs, Bohm et al. 2016). In many cases this unbalance was due to device disposal after use and failure of the user facility to return device to the manufacturer.

All failure modes described within an event description were accounted for; therefore, the potential for more than one failure mode recorded for a single report was possible. The number of reports with such occurrences was stated.

The frequency of failure modes was specified for each device. Failure modes were grouped by the phase of operation in which failure occurred. The phases of operation were identified based on the description of the adverse event in the reports. The phases of operation associated with tissue sealing devices included transition, activation, and post-op. The phases of operation associated with endoscopic staplers included reload, articulation, application, firing, cutting, removal, and staple line. Proportions were determined per failure mode relative to the total number of failure modes identified per device. A proportion of the number of failure modes that occurred in each phase of operation was also reported. The proportions were determined relative to the total number of failure modes per device versus the total sample per device because 
some reports involved more than one failure mode. Proportion percentages were reported to the nearest tenth decimal place.

\subsubsection{Medical Device Recalls}

Recalls associated with each device were retrieved from the FDA's Medical Device Recall database as another means to identify the presence of device related issues. The brand names were used in the recall query for all devices. Recall reports were organized by date, recall number, quantity in commerce, manufacturer reason for recall, and FDA determined cause. Consistencies in the reasons for recalls and device failures resulting in adverse events were identified for each device.

\section{$\underline{2.3 \text { Failure Mode Study Results }}$}

\subsubsection{Manufacturer Narrative Excerpts}

The following excerpts were taken directly from manufacturer narratives within adverse event reports that resulted in an injury (FDA 2017):

\section{Manufacturer Narrative - Ethicon ETS}

"Evaluation summary: the analysis results found that the device was received with the firing mechanism damaged and with a cartridge loaded in the device. The cartridge was received fully loaded with staples. No functional test could be performed with the device. The device was disassembled to verify the condition of the internal components and the firing trigger teeth were found broken. The returned cartridge was 
loaded into a test device and it fired, cut and formed all the staples as intended. Although no conclusion could be reached on how the device got damaged, it is possible that the device was fired on thicker tissue then intended causing the device to fail. It should be noted that at least a $100 \%$ inspection takes place during manufacturing to ensure the device meets the require specifications; in addition, a sample of the batch is inspected at fgqa. The manufacturing records were reviewed and no anomalies were found during the manufacturing process."

Manufacturer Narrative - Ethicon Harmonic Ace

"Should the information be provided later, a supplemental medwatch will be sent. The device was returned with the distal tip of the blade broken off and returned with the device. The remaining blade portion was scratched. The device was activated with the generator and an error code 5 was displayed. Probable causes of blade damage, including breakage, are external contact during pre-op or general use, blade contact with other devices, staples or clips during the procedure or using any means other than the blade wrench to attach or detach the blade. Once minor blade damage has occurred, subsequent activations may increase damage severity and result in an error code 5 or blade "lockout" later in the procedure, and continued usage can result in a broken blade."

Manufacturer Narrative - Olympus Thunderbeat

"Since the subject device was discarded by the user, the device was not returned to olympus medical systems corp. (omsc), therefore omsc could not evaluate the 
referenced device. Since the manufacture record of the subject device was unknown, the manufacture record that dated back the past six months from the delivery date to the user was reviewed, with no irregularities noted. Omsc could not determine the root cause conclusively. Based on the characteristic of the device, it is known that the temperature of the distal end of the device is high after activation and it caused thermal damage by contacting the device with the tissue. The instruction manual of the device already cautions; the grasping section and probe tip become hot due to extended ultrasonic output. Do not let it come in contact with tissues other than the target tissue."

The examples demonstrate the importance of medical device reporting and the subsequent user and manufacturer follow up given the type of information that the manufacturer narratives provide.

\subsubsection{Endoscopic Stapler Failure Modes and Recalls}

The types, frequency, and proportion of failure modes for the Ethicon ETS and Covidien Endo GIA staplers are shown in Tables 10 and 11, respectively. The frequency and proportion of failure modes per each phase of operation in which the failure occurred is also listed in the tables. 
Table 10. Endoscopic stapler phases and failure modes for ETS ( $n=111$ failures derived from 100 reports).

\begin{tabular}{|c|c|c|c|}
\hline $\begin{array}{l}\text { Phase of } \\
\text { Operation }\end{array}$ & $\begin{array}{c}\text { Frequency } \\
\text { (Proportion) }\end{array}$ & Failure Mode & $\begin{array}{c}\text { Frequency } \\
\text { (Proportion) }\end{array}$ \\
\hline \multirow{2}{*}{ Reload } & \multirow{2}{*}{$10(9.0 \%)$} & Reload fell out of or off of device & $7(6.3 \%)$ \\
\hline & & Reload malfunction & $3(2.7 \%)$ \\
\hline Articulation & $2(1.8 \%)$ & Articulation malfunction & $2(1.8 \%)$ \\
\hline Application & $2(1.8 \%)$ & Failure to open/close for application on tissue & $2(1.8 \%)$ \\
\hline \multirow{4}{*}{ Firing } & \multirow{4}{*}{$60(54.1 \%)$} & Malformed staples/Failure to form staples & $17(15.3 \%)$ \\
\hline & & Failure to fire (at all/completely/properly) & $9(8.1 \%)$ \\
\hline & & General misfire (nonspecific) & $14(12.6 \%)$ \\
\hline & & Failure to deploy staples (at all/on both sides) & $20(18 \%)$ \\
\hline \multirow{2}{*}{ Cutting } & \multirow{2}{*}{$3(2.7 \%)$} & Unintentionally nicked tissue & $0(0.0 \%)$ \\
\hline & & Failure to cut (at all/completely/properly) & $3(2.7 \%)$ \\
\hline \multirow{2}{*}{ Removal } & \multirow{2}{*}{$21(18.9 \%)$} & Failure to release & $20(18.0 \%)$ \\
\hline & & Difficult release & $1(0.9 \%)$ \\
\hline \multirow{2}{*}{ Staple line } & \multirow{2}{*}{$13(11.7 \%)$} & Staple line failure & $6(5.4 \%)$ \\
\hline & & Staple line leak post-op & $7(6.3 \%)$ \\
\hline
\end{tabular}


Table 11. Endoscopic stapler phases and failure modes for the Endo GIA ( $n=113$ failures derived from 100 reports).

\begin{tabular}{|c|c|c|c|}
\hline $\begin{array}{l}\text { Phase of } \\
\text { Operation }\end{array}$ & $\begin{array}{c}\text { Frequency } \\
\text { (Proportion) }\end{array}$ & Failure Mode & $\begin{array}{c}\text { Frequency } \\
\text { (Proportion) }\end{array}$ \\
\hline \multirow{2}{*}{ Reload } & \multirow{2}{*}{$6(5.3 \%)$} & Reload fell out of or off of device & $3(2.7 \%)$ \\
\hline & & Reload malfunction & $3(2.7 \%)$ \\
\hline Articulation & $0(0.0 \%)$ & Articulation malfunction & $0(0.0 \%)$ \\
\hline Application & $4(3.5 \%)$ & Failure to open/close for application on tissue & $4(3.5 \%)$ \\
\hline \multirow{4}{*}{ Firing } & \multirow{4}{*}{$58(51.3 \%)$} & Malformed staples/Failure to form staples & $30(26.5 \%)$ \\
\hline & & Failure to fire (at all/completely/properly) & $11(9.7 \%)$ \\
\hline & & General misfire (nonspecific) & $5(4.4 \%)$ \\
\hline & & Failure to deploy staples (at all/on both sides) & $12(10.6 \%)$ \\
\hline \multirow{2}{*}{ Cutting } & \multirow{2}{*}{$6(5.3 \%)$} & Unintentionally nicked tissue & $2(1.8 \%)$ \\
\hline & & Failure to cut (at all/completely/properly) & $4(3.5 \%)$ \\
\hline \multirow{2}{*}{ Removal } & \multirow{2}{*}{$23(20.4 \%)$} & Failure to release & $22(19.5 \%)$ \\
\hline & & Difficult release & $1(0.9 \%)$ \\
\hline \multirow{2}{*}{ Staple line } & \multirow{2}{*}{$16(14.2 \%)$} & Staple line failure & $6(5.3 \%)$ \\
\hline & & Staple line leak post-op & $10(8.8 \%)$ \\
\hline
\end{tabular}

The number of reports for the ETS and Endo GIA staplers that involved more than one failure mode was 11 and 13 , respectively. It should be noted that a multitude of events that reported firing phase issues also reported issues with removal for the same device incident $(n=7)$. The proportion of failures for each phase is shown per device in Figure 4. 


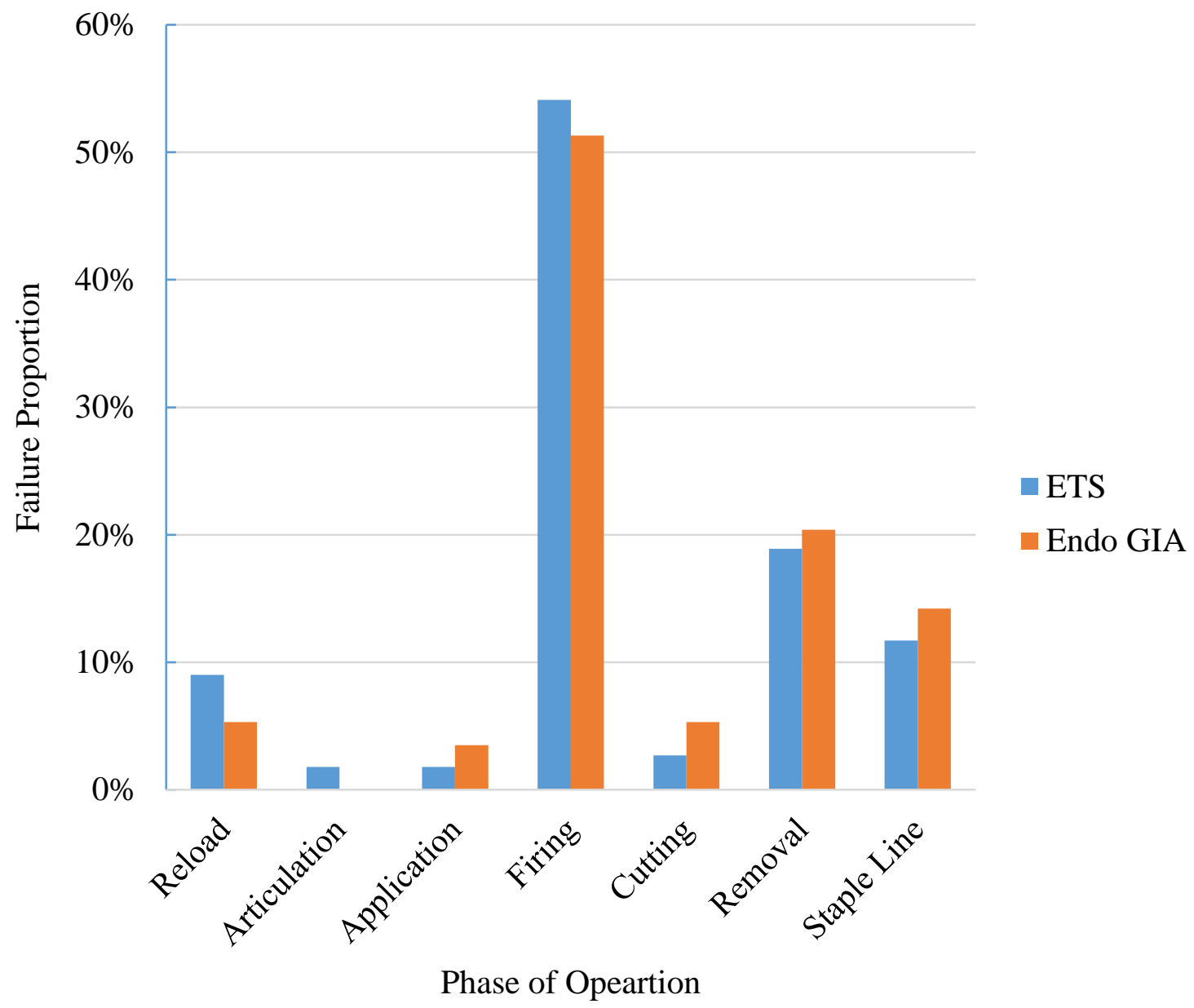

Figure 4. Proportion of failures per phase and device brand (ETS n=113, Endo GIA $\mathrm{n}=111)$.

The recalls associated with the Ethicon ETS stapler during the study period are shown in Table 12. 
Table 12. Ethicon ETS recalls.

\begin{tabular}{|c|c|c|c|c|}
\hline Date Posted & Recall No. & $\begin{array}{l}\text { Quantity in } \\
\text { Commerce }\end{array}$ & Manufacturer Reason for Recall & $\begin{array}{c}\text { FDA } \\
\text { Determined } \\
\text { Cause }\end{array}$ \\
\hline \multirow{2}{*}{ Oct-20-2016 } & Z-0295-2017 & 105 & \multirow{2}{*}{$\begin{array}{l}\text { Quality control identified a } \\
\text { component issue where the pinion } \\
\text { gear in the device could fail under } \\
\text { extreme use cases. If this condition } \\
\text { occurs, staples will be formed past } \\
\text { the cut line and the device can be } \\
\text { opened and removed from the } \\
\text { patient. The firing stroke may be } \\
\text { interrupted and the knife may not } \\
\text { fully return to the home position } \\
\text { potentially exposing the healthcare } \\
\text { professional to a sharps injury. }\end{array}$} & \multirow[t]{2}{*}{ Device Design } \\
\hline & Z-0296-2017 & 213 & & \\
\hline Jul-24-2015 & Z-2229-2015 & 3 & $\begin{array}{l}\text { EXP did not register as a medical } \\
\text { device establishment, list the } \\
\text { devices being recalled, or establish } \\
\text { its own quality system and instead } \\
\text { relied on the fact that its vendors } \\
\text { were registered and had their own } \\
\text { quality systems. }\end{array}$ & Other \\
\hline Sep-12-2011 & Z-3187-2011 & 27,582 & $\begin{array}{l}\text { Ethicon Endo-Surgery initiated a } \\
\text { voluntary global recall for specific } \\
\text { production lots of ENDOPATH } \\
\text { ETS Compact Flex } 45 \mathrm{~mm} \\
\text { articulating linear cutters because } \\
\text { the possibility exists that damage to } \\
\text { the blister pack may have } \\
\text { compromised the sterility of the } \\
\text { device. }\end{array}$ & Packaging \\
\hline $\begin{array}{c}\text { Nov-15- } \\
2010\end{array}$ & Z-0361-2011 & 4,113 & $\begin{array}{l}\text { The mechanism that connects the } \\
\text { articulation joint to the device shaft } \\
\text { may cause the jaws of the cutters to } \\
\text { remain closed and clamped down on } \\
\text { tissue after the device is fired. If the } \\
\text { jaws of the device remain clamped } \\
\text { down on the tissue, there is a risk } \\
\text { the surgery will need to be modified } \\
\text { to remove the device. This may lead } \\
\text { to an increase in procedure time, } \\
\text { tissue manipulation, and a change in } \\
\text { t... }\end{array}$ & Other \\
\hline $\begin{array}{l}\text { Dec-17- } \\
2003\end{array}$ & Z-0212-04 & 16,962 & $\begin{array}{l}\text { A defective articulation band may } \\
\text { result in improper staple formation } \\
\text { with possible hematosis. }\end{array}$ & $\begin{array}{l}\text { Non- } \\
\text { conforming } \\
\text { Material/ } \\
\text { Component } \\
\end{array}$ \\
\hline
\end{tabular}

The recalls associated with Endo GIA reloads and handles during the study period are shown in Table 13. The recalls associated with both endoscopic staplers were 
classified as Class II, which the FDA defines as a "situation in which use of or exposure to a violative product may cause temporary or medically reversible adverse health consequences or where the probability of serious adverse health consequences is remote" (FDA 2009). 
Table 13. Covidien Endo GIA recalls.

\begin{tabular}{|c|c|c|c|c|}
\hline Date Posted & Recall No. & $\begin{array}{l}\text { Quantity in } \\
\text { Commerce }\end{array}$ & $\begin{array}{c}\text { Manufacturer Reason for } \\
\text { Recall }\end{array}$ & $\begin{array}{c}\text { FDA } \\
\text { Determined } \\
\text { Cause }\end{array}$ \\
\hline \multirow{4}{*}{ Feb-18-2016 } & Z-0819-2016 & 364,768 & \multirow{4}{*}{$\begin{array}{l}\text { Staplers fail to fire or } \\
\text { partially fire and reports of } \\
\text { the instrument articulating } \\
\text { lever disengaging during } \\
\text { use }\end{array}$} & \multirow{4}{*}{ Process control } \\
\hline & Z-0820-2016 & $1,102,166$ & & \\
\hline & Z-0821-2016 & 327,797 & & \\
\hline & Z-0822-2016 & 2,711 kits & & \\
\hline Apr-05-2013 & Z-1063-2013 & 6,751 & $\begin{array}{l}\text { Single Use Loading Unit } \\
\text { contained two staples } \\
\text { loaded in each cartridge } \\
\text { pocket and may result in } \\
\text { difficulty firing and } \\
\text { removing the device from } \\
\text { the application site, which } \\
\text { may require medical } \\
\text { intervention }\end{array}$ & Process control \\
\hline \multirow{8}{*}{ Sep-21-2012 } & Z-2432-2012 & 433,528 & \multirow{8}{*}{$\begin{array}{l}\text { Duet TRS may have the } \\
\text { potential to injure adjacent } \\
\text { anatomical structures which } \\
\text { may result in life } \\
\text { threatening post-operative } \\
\text { complications }\end{array}$} & \multirow{8}{*}{ Device Design } \\
\hline & Z-2433-2012 & 94,301 & & \\
\hline & Z-2434-2012 & 4,867 & & \\
\hline & Z-2435-2012 & 104,355 & & \\
\hline & Z-2436-2012 & 22,218 & & \\
\hline & Z-2437-2012 & 268,022 & & \\
\hline & Z-2438-2012 & 15,936 & & \\
\hline & Z-2439-2012 & 135,712 & & \\
\hline Mar-19-2012 & Z-1227-2012 & 1,158 & $\begin{array}{l}\text { Missing component results } \\
\text { in the stapler not firing. }\end{array}$ & Process control \\
\hline \multirow{8}{*}{ Feb-13-2012 } & Z-0991-2012 & 5,102 & \multirow{8}{*}{$\begin{array}{l}\text { Duet TRS Straight and } \\
\text { Articulating Single Use } \\
\text { (SULU) Loading Staplers } \\
\text { used in thoracic surgery } \\
\text { may result in serious injury } \\
\text { or death }\end{array}$} & \multirow{8}{*}{$\begin{array}{l}\text { Under } \\
\text { Investigation by } \\
\text { firm }\end{array}$} \\
\hline & Z-0992-2012 & 91,813 & & \\
\hline & Z-0993-2012 & 3,391 & & \\
\hline & Z-0994-2012 & 97,326 & & \\
\hline & Z-0995-2012 & 12,961 & & \\
\hline & Z-0996-2012 & 231,075 & & \\
\hline & Z-0997-2012 & 9,687 & & \\
\hline & Z-0998-2012 & 220,622 & & \\
\hline \multirow{8}{*}{ Sep-08-2011 } & Z-3164-2011 & 4,362 & \multirow{8}{*}{$\begin{array}{l}\text { Sterility of the device is } \\
\text { compromised due to a } \\
\text { breach in the sterile } \\
\text { packaging }\end{array}$} & \multirow{8}{*}{ Storage } \\
\hline & Z-3165-2011 & 7,4865 & & \\
\hline & Z-3166-2011 & 2,928 & & \\
\hline & Z-3167-2011 & 2,928 & & \\
\hline & Z-3168-2011 & 11,336 & & \\
\hline & Z-3169-2011 & 184,773 & & \\
\hline & Z-3170-2011 & 7,842 & & \\
\hline & Z-3171-2011 & 168,427 & & \\
\hline
\end{tabular}




\subsubsection{Energy-Based Tissue Sealer Failure Modes and Recalls}

The types, frequency, and proportion of failure modes for the Olympus

Thunderbeat and Ethicon Harmonic Ace tissue sealers are shown in Tables 14 and 15, respectively. The frequency and proportion of failure modes per each phase of operation in which the failure occurred is also listed in the tables.

Table 14. Tissue sealer phases and failure modes for the Olympus Thunderbeat $(\mathrm{n}=81$ failures derived from 81 reports).

\begin{tabular}{|c|c|c|c|}
\hline Phase & $\begin{array}{c}\text { Frequency } \\
\text { (Proportions) }\end{array}$ & Failure Mode & $\begin{array}{c}\text { Frequency } \\
\text { (Proportions) }\end{array}$ \\
\hline \multirow{7}{*}{ Activation } & \multirow{7}{*}{$36(44.4 \%)$} & Unspecific error resulting in bleeding & $1(1.2 \%)$ \\
\hline & & Broken device - blade or probe & $11(13.6 \%)$ \\
\hline & & Broken device - tissue pad & $7(8.6 \%)$ \\
\hline & & Failure to seal/failure to seal properly & $6(7.4 \%)$ \\
\hline & & Direct application & $7(8.6 \%)$ \\
\hline & & Thermal spread & $2(2.5 \%)$ \\
\hline & & Insulation Failure & $2(2.5 \%)$ \\
\hline \multirow{5}{*}{ Transition } & \multirow{5}{*}{$30(37.0 \%)$} & Residual heat & $23(28.4 \%)$ \\
\hline & & Broken device - blade or probe & $1(1.2 \%)$ \\
\hline & & Broken device - tissue pad & $0(0.0 \%)$ \\
\hline & & Inadvertent application & $5(6.2 \%)$ \\
\hline & & Installation error; electric shock & $1(1.2 \%)$ \\
\hline \multirow{2}{*}{ Post-op } & \multirow{2}{*}{$15(18.5 \%)$} & Seal failure with bleeding/leakage & $15(18.5 \%)$ \\
\hline & & Tissue injury detected after device use & $0(0.0 \%)$ \\
\hline
\end{tabular}


Table 15. Tissue sealer phases and failure modes for the Ethicon Harmonic Ace ( $n=102$ failures derived from 100 reports).

\begin{tabular}{|c|c|c|c|}
\hline Phase & $\begin{array}{c}\text { Frequency } \\
\text { (Proportions) }\end{array}$ & Failure Mode & $\begin{array}{c}\text { Frequency } \\
\text { (Proportions) }\end{array}$ \\
\hline \multirow{7}{*}{ Activation } & \multirow{7}{*}{$47(46.1 \%)$} & Unspecific error resulting in bleeding & $4(3.9 \%)$ \\
\hline & & Broken device - blade or probe & $10(9.8 \%)$ \\
\hline & & Broken device - tissue pad & $14(13.7 \%)$ \\
\hline & & Failure to seal/failure to seal properly & $16(15.7 \%)$ \\
\hline & & Direct application & $3(2.9 \%)$ \\
\hline & & Thermal spread & $0(0.0 \%)$ \\
\hline & & Insulation Failure & $0(0.0 \%)$ \\
\hline \multirow{5}{*}{ Transition } & \multirow{5}{*}{$24(23.5 \%)$} & Residual heat & $16(15.7 \%)$ \\
\hline & & Broken device - blade or probe & $3(2.9 \%)$ \\
\hline & & Broken device - tissue pad & $4(3.9 \%)$ \\
\hline & & Inadvertent application & $1(1.0 \%)$ \\
\hline & & Installation error; electric shock & $0(0.0 \%)$ \\
\hline \multirow{2}{*}{ Post-op } & \multirow{2}{*}{$31(30.4 \%)$} & Seal failure with bleeding/leakage & $29(28.4 \%)$ \\
\hline & & Tissue injury detected after device use & $2(2.0 \%)$ \\
\hline
\end{tabular}

The activation phase consists of intentional grasping, cutting, and/or sealing of tissue. The transition phase refers to device preparation, insertion, removal, and internal and external transitions during surgery. Post-op refers to failures that occur after the surgery has been completed. Two Thunderbeat reports did not contain sufficient descriptions to identify the specific failure modes; though, it was inferred that both occurred post-op. Two Harmonic reports contained more than one type of failure mode.

The proportion of failures for each phase is shown per device in Figure 5. 


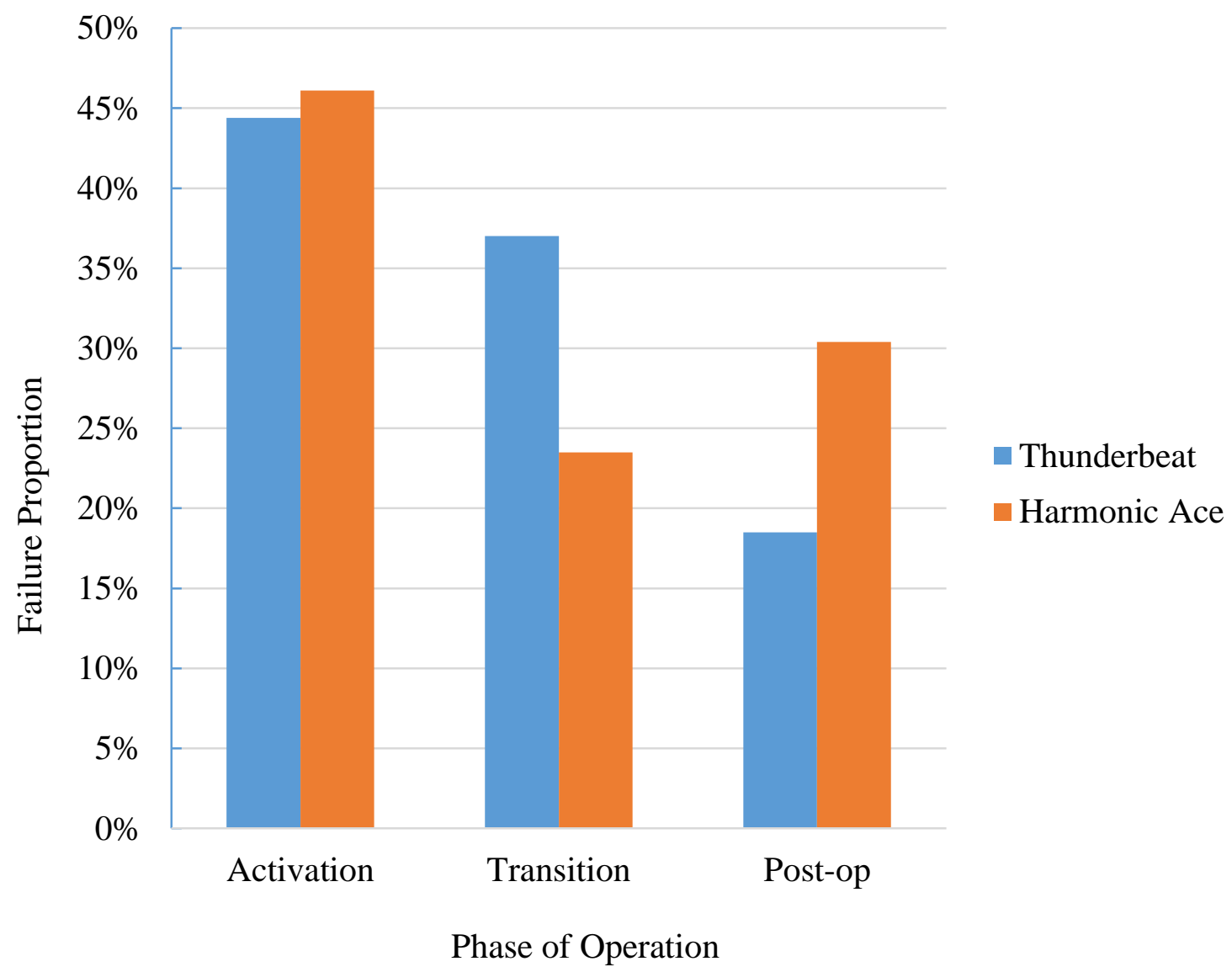

Figure 5. Proportion of failures per phase and device brand (Thunderbeat $n=81$, Harmonic $n=102$ ).

The recall associated with the Olympus Thunderbeat during the study period are shown in Table 16. 
Table 16. Olympus Thunderbeat recall.

\begin{tabular}{|c|c|c|l|c|}
\hline Date Posted & Recall No. & $\begin{array}{c}\text { Quantity in } \\
\text { Commerce }\end{array}$ & $\begin{array}{c}\text { Manufacturer Reason } \\
\text { for Recall }\end{array}$ & $\begin{array}{c}\text { FDA } \\
\text { Determined } \\
\text { Cause }\end{array}$ \\
\hline & Z-0432-2014 & 8,709 & $\begin{array}{l}\text { Complaints of damage to } \\
\text { various models of } \\
\text { Thunderbeat Hand } \\
\text { Instruments during } \\
\text { surgical procedures, } \\
\text { including cracking, } \\
\text { breaking, or deformation } \\
\text { of the components of the } \\
\text { probe tip or jaw. }\end{array}$ & Use error \\
\hline
\end{tabular}

The recalls associated with the Ethicon Harmonic Ace tissue sealer during the study period are shown in Table 17.

Table 17. Ethicon Harmonic Ace recalls.

\begin{tabular}{|c|c|c|l|c|}
\hline Date Posted & Recall No. & $\begin{array}{l}\text { Quantity in } \\
\text { Commerce }\end{array}$ & $\begin{array}{l}\text { Manufacturer Reason for } \\
\text { Recall }\end{array}$ & $\begin{array}{c}\text { FDA } \\
\text { Determined } \\
\text { Cause }\end{array}$ \\
\hline Nov-16-2015 & Z-0278-2016 & 441,490 & $\begin{array}{l}\text { Complaints of holes in } \\
\text { Tyvek which can } \\
\text { compromise the sterile } \\
\text { barrier provided by the } \\
\text { packaging. }\end{array}$ & Device design \\
\hline Feb-13-2015 & Z-1117-2015 & 12,467 - both sizes & $\begin{array}{l}\text { Internal labeling review } \\
\text { found that the [Instructions } \\
\text { for Use] IFU for the } \\
\text { Harmonic Ace incorrectly } \\
\text { instructs the user on how to } \\
\text { manually open the grips. }\end{array}$ & Error in \\
& Z-1118-2015 & 12,467 - both sizes & labeling \\
\hline July-01-2013 & Z-1644-2013 & \multirow{2}{*}{3} & $\begin{array}{l}\text { The seal which maintains a } \\
\text { sterile barrier for } \\
\text { reprocessed medical devices } \\
\text { became compromised to the } \\
\text { point where product may } \\
\text { fall out of the pouch. }\end{array}$ & process control \\
& & & \\
\hline
\end{tabular}

The recalls associated with both tissue sealers were classified as Class II. 


\subsection{Failure Mode Study Discussion}

The objective of Specific Aim 1 was to describe the types of failure modes shown to be associated with endoscopic staplers and energy-based tissues sealing devices used in MIS based on reported adverse events. The proportions of failure modes and phases in which failure occurred were calculated relative to the total number of failures identified per each device.

\subsubsection{Endoscopic Stapler Failure Mode Discussion}

The phases in which failure occurred most frequently were firing (ETS - 54.1\%, Endo GIA - 51.3\%), removal (ETS - 18.9\%, Endo GIA - 20.4\%), and staple line (ETS $11.7 \%$, Endo GIA - 14.2\%). Failures associated with the endoscopic staplers occurred most frequently during the firing phase. Within the firing phase, the most common failure modes associated with endoscopic staplers were the failure to deploy staples at all or on both sides (ETS - 18.0\%, Endo GIA - 10.6\%) and malformed staples or the failure to form staples (ETS - 15.3\%, Endo GIA - 26.5\%). Of the failures during firing, the Endo GIA resulted in more malformed staples or failure to form staples compared to the ETS, while the ETS resulted in more failures to deploy staples at all or on both sides of tissue. Overall, malformed staples or a failure to form staples contributed to the most adverse events occurring during firing. The most common failure mode within the removal phase was the failure of the device to release from tissue after firing. $18.0 \%$ of Ethicon ETS and $19.5 \%$ of Covidien Endo GIA failures involved the failure to release. 
Issues encountered with the staple line involved staple line failure (ETS - 5.4\%, Endo GIA $-5.3 \%$ ) and staple line leaks post-op (ETS - 6.3\%, Endo GIA - 8.8\%).

The most common phases in which failure occurred and the most common failures modes within each phase identified in this study were consistent with those recognized throughout the scientific literature (Deng, Meng et al. 2002, Champion and Williams 2003, Hamilton, Sims et al. 2003, Marshall, Srivastava et al. 2003, Brown and Woo 2004, Hassouna and Manikandan 2012, Mansour, El-Nashar et al. 2014). Brown and Woo reviewed 112 FDA MAUDE death reports in their investigation of fatalities associated with surgical staplers. The authors found the failure to fire properly, ruptured staple lines, and failure to form staples to be the most common types of stapler issues that resulted in fatalities (Brown and Woo 2004). Deng et al. reviewed approximately 460 urologic laparoscopic cases in order to characterize the problems with linear cutting staplers and concluded that the most common and morbid aspects of stapling device failure were abnormal firing of the stapler and improper staple formation (malformed staples) (Deng, Meng et al. 2002). An investigation into Endo-GIA malfunctions during laparoscopic nephrectomy that were reported to the FDA MAUDE database showed the most common mechanisms of failure involved malformed or leaking staple line after firing and failure of the device to release from tissue (Mansour, El-Nashar et al. 2014). Similarly, the two most common mechanisms of endoscopic stapler failure during laparoscopic renal surgery were determined to be inadequate staple lines and failure to release from tissue, which resulted in a high percentage of severe hemorrhage and conversions to open surgery (Hassouna and Manikandan 2012). Outcomes of gastric bypass surgery have shown staple line failures and anastomotic leaks to be a major cause 
of patient morbidity and death (Champion and Williams 2003, Hamilton, Sims et al. 2003, Marshall, Srivastava et al. 2003). While the most common types of endoscopic failure were shown to vary per procedural application in the reviews discussed, the phases of operation in which failure most frequently occurred were consistent with the top three identified in this study: firing, removal, and staple line.

The ETS stapler was associated with the most reload failures (9.0\%), some of which the reload fell completely out of the device and into the patient (6.3\%). This type of failure can lead to serious consequences such as the cutting of tissue without simultaneous deployment of staples, which was reported with user experience during laparoscopic Roux-en-Y gastric bypass (Champion and Williams 2003). Despite similarity in the specific function executed, variations in failure frequency between the devices are potentially due to the differences in reloading mechanisms inherent to each device's design. The ETS stapler is supplied as a full stapler that requires insertion of individual reloads, also referred to as cartridges, into the elongate channel. After the ETS stapler is fired, it requires a new reload in order to be used again in the same procedure. The reload is inserted into place via a snap fit type of mechanism. The Endo GIA also involves two separate components, yet it is supplied as a handle with a shaft portion that connects to a separate reload portion via an interlocking mechanism. Replacing an Endo GIA reload provides new jaws, distal articulation components, and knife for the next fire during the same procedure. These differences are discussed further in Section 4.3.1.

Endoscopic staplers resulted in more reports that involved failure in two phases relative to tissue sealers. It should be noted that a multitude of events that reported firing phase issues also reported issues with removal for the same device incident $(n=7)$. Such a 
trend suggests that the failures associated with the firing sequence, whether device or user related, may result in a failure during removal. Removal was the second most common phase of operation in which failure occurred and consisted mainly of stapler failure to release from tissue after firing. When a device fails to release, the surgeon must deploy unfavorable tactics, which includes manually cutting adjacent tissue or creating another port for application of another stapling device in order to remove the malfunctioning device (Kwazneski, Six et al. 2012). Kwazneki II et al. found that of the 44 minimally invasive program directors that completed their survery, $66 \%$ of survey participants experienced an incident where a linear stapler would not release after application, $25 \%$ of survey participants noted having to significantly alter the planned procedure due to a device failure, and $30 \%$ of survey participants stated that they did not receive useful feedback from the manufacturer despite making contact (Kwazneski, Six et al. 2012).

In a 2004 review of surgical stapler-associated fatalities and adverse events reported to the FDA, it was found that similar failures identified in the death reports were also responsible for the majority of adverse events that did not result in a death (i.e. injury and malfunctions) (Brown and Woo 2004). Such a finding suggests that if device malfunctions are either prevented or handled properly when they occur then, as a result, death and injuries can be prevented. The final outcome, whether injury or death, can relate back to a user's familiarity with device performance and failure in addition to their response to an adverse event (Mansour, El-Nashar et al. 2014). Specifically, the methods deployed in reaction to a failure may affect whether or not a malfunction causes an injury or death. Also, preventative techniques play an important role in the severity of outcomes of adverse events (Baker, Foote et al. 2004, Fuller, Paull et al. 2014, Mansour, 
El-Nashar et al. 2014), which is emphasized further in the assessment of failure mode outcome severity in Chapter II.

Determining whether an endoscopic staler failure is user or device related is difficult and cannot always be derived from the report event descriptions. Consider the failure mode involving the failure of the device to properly form staples. Malformed staples can result from improper contact of the unformed staples with the anvil pockets. Specifically, Chekan and Whelan list tissue and staple related causes of malformed staples as they relate to the staple legs (Chekan and Whelan 2014). The tissue characteristics affecting the formation of staples include tissue thickness and viscosity, while the staple properties include the staple thickness, height, bending characteristics, and type of metal (Chekan and Whelan 2014). Additionally, firing over clips, or a similar obstruction, can result in malformed staples, a failure to form staples, and/or the failure of a device to release from tissue after firing (Mansour, El-Nashar et al. 2014, Riggs, Bohm et al. 2016). While this is specific to a user error, instances involving firing over a clip may not be adequately described (i.e. presence of the clip was not included in the adverse event report). The device could also cause malformed staples due to a manufacturing defect, such as stapler misalignment (Fuller, Paull et al. 2014). Typically, the event descriptions within the FDA MAUDE database reports are not specific enough to identify the root cause of failure mode that results in an adverse event outcome.

Recalls involving the endoscopic staplers were retrieved over a period ranging from 2003 to 2016. A total of 36 recall numbers were retrieved in the search, of which 30 were related to the Covidien Endo GIA and 6 were related to the Ethicon ETS. Of the five manufacturer reasons for recall associated with the Ethicon ETS stapler recalls, three 
are consistent with adverse event outcomes. The recall reason that states "the mechanism that connects the articulation joint to the device shaft may cause the jaws of the cutters to remain closed and clamped down on tissue after the device is fired; if the jaws of the device remain clamped down on tissue, there is a risk that the surgery will need to be modified to remove the device; this may lead to an increase in procedure time, tissue manipulation, and a change in t...” accounted for one recall (Z-0361-2011) and a total quantity in commerce of 4,113 . This recall reason was consistent with the failure of the device to release from tissue after it is fired as identified in this study. The recall reason stating "a defective articulation band may result in improper staple formation with possible hematosis" accounted for one recall (Z-0212-04) and a total quantity in commerce of 16,962. This recall reason was consistent with the following failure modes identified: articulation malfunction, malformed staples, and failure to form staples.

One recall associated with the Ethicon ETS stapler did not relate to a specific failure mode identified herein but demonstrated the potential for device related issues to cause adverse event outcomes. The recall reason stating "quality control identified a component issue where the pinion gear of the device could fail under extreme use cases; if this condition occurs, staples can be formed past the cut line and the device can be opened and removed from the patient; the firing stroke may be interrupted and the knife may not fully return to the home position potentially exposing the healthcare professional to a sharp injury" accounted for two recalls (Z-0295-2017, Z-0296-2017) and a total quantity in commerce of 318 . This recall reason did not specifically state a failure mode identified herein. Though, it did mention a specific device related failure that can cause improper functioning and injury. Further, the failure of the knife to return to its home 
position has the potential to affect the removal of the device from tissue given that the knife must be pulled back to its original position enough in order for the jaws to open and release.

Of the six manufacturer reasons for recall associated with the Covidien Endo GIA stapler recalls, five were consistent with adverse event outcomes. The recall reason stating "staplers fail to fire or partially fire and reports of the instrument articulating lever disengaging during use" accounted for four recalls (Z-0819-2016, Z-0820-2016, Z08-212016, Z-0822-2016) and a total quantity in commerce of 1,797,442. This recall reason was consistent with the following failures mode identified herein: articulation malfunction, failure to fire (at all/completely/properly), and failure to deploy staples (at all/on both sides). The recall reason stating "single use loading unit contained two staples loaded in each cartridge pocket and may result in difficulty firing and removing the device from the application site, which may require medical intervention" accounted for one recall (Z-1063-2013) and a total quantity in commerce of 6,751. This recall reason was consistent with the following failure modes identified: failure to fire properly, failure to release, and difficult release. The recall reason stating "missing component results in the stapler not firing" accounted for one recall (Z-1227-2012) and a total quantity in commerce of 1,158 . This recall reason was consistent with the failure to fire.

Recalls associated with the Covidien Endo GIA were identified that did not relate to a specific failure mode identified herein but demonstrate the potential for device related issues to cause adverse event outcomes. The recall reason stating "duet TRS may have the potential to injure adjacent anatomical structure which may result in life threatening post-operative complications" accounted for eight recalls (Z-2432-2012, Z- 
2439-2012) and a total quantity in commerce of $1,078,939$. The recall reason was not specific as to what aspect of the device could potentially cause injury to adjacent tissue and did not directly relate to the failure modes identified. However, it is an important recall reason to recognize given the magnitude of the quantity recalled and it highlights the potential for device related causes of adverse event outcomes. The recall reason that states "duet TRS straight and articulating single use (SULU) loading staplers used in thoracic surgery may result in serious injury or death" accounted for eight recalls (Z0991-2012, Z-0992-2012, Z-0993-2012, Z-0994-2012, Z-0995-2012, Z-0996-2012, Z0997-2012, Z-0998-2012) and a total quantity in commerce of 671,977. This was another recall reason that did not directly relate to a specific failure mode identified herein. The recall reason was not specific as to what feature of the device is responsible for a potential serious injury or death, yet it also demonstrates the possibility of adverse event outcomes due to device related issues.

Overall, a total quantity in commerce of 3,577,660 were recalled for reasons associated with endoscopic stapler device related issues that could potentially cause adverse event outcomes. Of this total, the Covidien Endo GIA recalls $(3,556,267)$ involved a higher quantity in commerce than the Ethicon ETS recalls $(4,113)$. The reasons for endoscopic stapler product recall as identified by the FDA were consistent with the failure modes and failure mode consequences identified in the adverse event reports. Specifically, the recall reasons coincide with two of the most frequent failure modes for endoscopic staplers which were malformed staples/failure to form staples and failure to release from tissue. In addition, the reasons for recall include the potential of 
post-operative complications and serious injury or death, which was consistent with the nature of the failure outcomes identified in the adverse event reports.

The number of recalls associated with the endoscopic staplers that correspond to the adverse event outcomes indicate that device failure shares responsibility, which is consistent with previous findings in the literature (Brown and Woo 2004, Kwazneski, Six et al. 2012, Fuller, Paull et al. 2014). Kwazneski et al. state that both the number of recalls and the number of endoscopic stapling problems experienced by users suggests a redesign and remanufacture of the staplers could positively impact the safety profile of the devices (Kwazneski, Six et al. 2012). Further, it is likely that all issues associated with these devices are underreported to the FDA, which results in an underestimation of the extent of the failures and the corresponding root causes (Brown and Woo 2004).

Fuller et al. assessed 25 stapling-related root causes from the Veterans Health Administration (VHA) and mapped them to the following: 10 were environment issues (40\%), seven were user issues (28\%), five were task issues (20\%), and three were equipment issues (12\%) (Fuller, Paull et al. 2014). Environmental issues include a lack of surgical team educational opportunities, overscheduling of cases, and lack of a standardized procedure to attain blood products in an emergency. User issues included firing a misaligned stapler and lack of familiarity of the device. Poor visualization of the target tissue and friable tissue characteristics were cited to be task issues. Finally, equipment issues were defined as misalignment of the stapler and misfire (Fuller, Paull et al. 2014). This brings attention to factors other than solely user and device related issues. Fuller et al. also provided an extensive list of recommendations in order to prevent or aid in recovery from endoscopic stapler adverse events. Recommendations are listed for 
before surgery, during surgery, and after an adverse event and include the following examples for each stage, respectively (Fuller, Paull et al. 2014):

- "Implement directed systematic training on surgical devices for all members of the surgical team to increase familiarity with the device, its approved use scenarios, and known characteristics of device malfunction.

- Utilize a standard procedure for tracking information about the stapler during the procedure, including number of firings, loadings, and reloadings.

- If stapler malfunction is noted when the stapler is still locked on tissue, clamp or litigate the vessel before releasing the stapler."

The recommendations can assist users with various aspects of endoscopic stapling devices and also improve the reporting of adverse events to the FDA by suggesting implementation of a standardized procedure for investigations of device misfires (Fuller, Paull et al. 2014). Such practices may also assist in determining the root cause of failure, whether user, environment, task, or device related.

\subsubsection{Energy-Based Tissue Sealer Failure Mode Discussion}

The majority of tissue sealing device failures occurred during the activation phase of operation for both devices (Thunderbeat $-44.4 \%$, Harmonic Ace $-46.1 \%$ ). The most common failure mode during Thunderbeat activation was a broken probe (13.6\%), whereas the most common failure mode during Harmonic Ace activation was the failure 
to seal or seal properly $(15.7 \%)$. The second most common phases of operation in which failure occurred for the Thunderbeat and Harmonic Ace were transition (37\%) and postop $(30.4 \%)$, respectively. Within the transition phase, residual heat was the most common failure mode associated with the Thunderbeat (28.4\%). The most common failure mode that occurred post-op associated with the Harmonic Ace was seal failure with bleeding or leakage $(28.4 \%)$. The phase of operation associated with the Thunderbeat in which the least amount of failures occurred was the post-op phase $(18.5 \%)$. The transition phase involved the least amount of failures associated with the Harmonic Ace $(23.5 \%)$.

Upon assessing the devices separately, the most common failure mode associated with the Thunderbeat was residual heat $(28.4 \%)$, whereas seal failure with bleeding or leakage was the most common failure mode associated with the Harmonic Ace (28.4\%). The second most common failure mode associated with the Thunderbeat was seal failure with bleeding and leakage (18.5\%). The second most common failure mode associated with the Harmonic Ace was tied between residual heat (15.7\%) and failure to seal or seal properly $(15.7 \%)$. Overall, the top two failure modes associated with both devices were residual heat and seal failure with bleeding or leakage.

Tissue sealing device failures induced by a broken device were more associated with the activation phase relative to the transition phase. The proportion of broken device failures involving the blade or probe was higher for both devices in the activation phase (Thunderbeat - 13.6\%, Harmonic Ace - 9.8\%) versus the transition phase (Thunderbeat $-1.2 \%$, Harmonic Ace $-2.9 \%$ ). Similarly, the proportion of broken device failures that involved the tissue pad were higher in the activation phase (Thunderbeat - 
8.6\%, Harmonic Ace $-13.7 \%$ ) versus the transition phase (Thunderbeat $-0.0 \%$, Harmonic Ace $-3.9 \%$ ). Of the broken device failures that occurred during activation, the Thunderbeat failures were more associated with the blade or probe component (13.6\%), while the Harmonic Ace was more associated with the tissue pad (13.7\%). The tissue pad and blade responsible for these failures are both essential components of the jaw portion that grasp and secure the tissue during activation. Proper delivery of thermal and mechanical energy to the tissue relies on these components. Per manufacturer notice and instruction, wear and separation of the tissue pad can be due to activation of the device while there is no tissue present in the grasping section. The user must avoid contact between the tissue pad and grasping section during activation to preserve the integrity of the distal tip components. Grasping tissue thicker than the specific range for the devices can also result in damage of the tissue pad and blade. The device must not come into contact with staples, clips, or other surgical instruments during activation.

The most common failure during transition was residual heat, which refers to injury caused by a heated electrode or blade after the completion of the activation cycle. Residual heat causes burns to surrounding internal organs $(n=20)$, external patient skin $(\mathrm{n}=10)$, and operating staff $(\mathrm{n}=9)$ when not handled cautiously during and after activation. The probe tip of the device and a portion of the distal end of the shaft becomes heated during and after prolonged activation. The Harmonic Ace and Thunderbeat reports resulted in the same number of residual heat injuries involving the patient's internal organs ( $\mathrm{n}=10$ for each device) and external skin ( $\mathrm{n}=5$ for each device). Eight residual heat injuries associated with the Thunderbeat device involved the operating staff, whereas only one of these incidents was associated with the Harmonic 
device. For both devices, seal failure with bleeding or leakage was the most common failure mode in the post-op phase of operation (Thunderbeat - 18.5\%, Harmonic Ace $28.4 \%)$.

The failure modes related to the Ethicon Harmonic Ace (ultrasonic energy source) and the Olympus Thunderbeat (ultrasonic and bipolar energy source) are in large part specific to their energy sources. This finding has been documented in scientific literature (Tucker and Voyles 1995, Overbey, Townsend et al. 2015). Overbey et al. analyzed reports from the FDA MAUDE and identified the common mechanisms leading to injury and death associated with various types of surgical energy-based device. The types of devices assessed in their investigation included traditional bipolar instruments, plasma beam monopolar devices, advanced bipolar devices, ultrasonic devices, radiofrequency/microwave ablation devices, and the monopolar "Bovie" instrument (Overbey, Townsend et al. 2015). While their proportions cannot be directly related to the proportions herein due to the range of device types assessed, the types of failures and outcomes shown to be associated with bipolar and ultrasonic energy devices were similar to those identified. Overbey et al. indicated that advanced bipolar tissue sealing device adverse event outcomes reported to the FDA MAUDE database mostly involved bleeding and thermal burns due to direct application (Overbey, Townsend et al. 2015). In addition, ultrasonic energy device adverse event outcomes were also most commonly associated with bleeding as a result of failure. Mechanical failure and thermal burn due to residual heat were associated with ultrasonic devices. These findings are consistent with the data presented herein considering that both tissue sealers assessed utilize ultrasonic energy. 
The bleeding associated with tissue sealing devices is concerning; however, it should be put into perspective when comparing devices across a range of applications since this failure consequence is inherent to the very function these devices carry out, which is to seal and transect vessels. While the specific failure modes were not identified by Overbey et al., the adverse event outcomes that involve bleeding are likely due to seal failure that involves bleeding or leakage, which was one of the top two failure modes identified across the Thunderbeat and Harmonic Ace reports.

Residual heat was identified by Overbey et al. as a mechanism of injury associated with ultrasonic energy, which is also supported elsewhere in scientific literature (Govekar, Robinson et al. 2011, Overbey, Townsend et al. 2015). Residual heat was one of the most common failure modes associated with the tissue sealers assessed in this study. Ultrasonic tissue transection is carried out via mechanical friction between the oscillating shaft of the device and the tissue. Inherent to this mechanism is an increase in temperature that is proportional to the time of activation. That is, the longer the device is active, the greater the distal tip temperature. Therefore, injury due to both residual heat and lateral thermal spread remain a concern (Lyons and Law 2013). The consequences of lateral thermal spread is a risk across all laparoscopic energy sources (Lyons and Law 2013). Ultrasonic and bipolar energy devices have evolved to include temperature regulation and control, yet the 2013 study by Lyons et al. states that the effectiveness and benefits of some of the various methods have yet to be proven in clinical trials (Lyons and Law 2013). These methods include adaptive technology that regulates energy delivery during activation and an audible feedback to the user indicating the device status through the activation stages via audible tone changes (Lyons and Law 2013). 
Relative to electrosurgical techniques that deliver energy through tissue, the advantages to devices utilizing ultrasonic energy include less tissue necrosis and charring, less smoke generation, and reduced lateral thermal spread (Entezari, Hoffmann et al. 2007, Lamberton, Hsi et al. 2008). In general, lateral thermal spread associated with ultrasonic energy has been shown to be relatively less (Hruby, Marruffo et al. 2007, Noble, Smart et al. 2011). However, a device's residual temperature of the device tip after activation is less with bipolar technology (Govekar, Robinson et al. 2011, Lyons and Law 2013). It is important to note that the extent of the effects of lateral thermal spread and residual heat associated with laparoscopic sealing technologies relies heavily on the operator. User experience and knowledge is critical, especially considering many of the failure modes are preventable. Instructions for ultrasonic device use indicate the possibility of residual heat and further emphasize the caution that must be implemented after activation. It is not advised to grasp other tissue immediately after an activation cycle. Care must be taken when handling the device after activation when inside a patient to avoid injury and burns to tissue. The surgical staff must also use caution when the device is handled outside of the patient, as the heated tip can still cause injury to skin (even through surgical gloves) if contact occurs. Users must be aware of the available features of their devices of choice in order to properly follow the instructions; for example, if the equipment generates audible tones indicating the stages of device activation, the user must be aware of what each tone indicates and signals regarding the state of the tissue.

The prevalence of failures caused by operator error should be addressed by both users and manufacturers of the devices. Direct application (in which the active electrode/ 
probe directly causes unintended thermal injury to adjacent tissue during activation), inadvertent application, and injury from residual heat are typically caused by wellfunctioning devices that are being handled incorrectly at the time of injury (Alkatout, Schollmeyer et al. 2012, Jones, Brunt et al. 2015). The level of understanding held by the user relating to all aspects of their choice of tissue sealing devices must be considered when assessing adverse events, especially their knowledge related to the generation of current and heat via electrical and mechanical energy. Users have a responsibility to adhere to instructions for use and warnings provided; however, device manufacturers have a separate responsibility to investigate, acknowledge, and address operational aspects of the device that inhibit proper use. The findings of this study support the need for a standardized curriculum for users to fill the knowledge gap related to surgical energy-based devices (Tucker and Voyles 1995, Harrell and Kopps 1998, Feldman, Fuchshuber et al. 2012, Feldman, Brunt et al. 2013, Watanabe, Kurashima et al. 2016).

Surgeons are tasked with determining the most appropriate device to use for a specific procedure and tissue type. It can be difficult for a user to make a properly informed and objective decision regarding the most appropriate laparoscopic energy source provided that some studies published in medical literature are sponsored by device manufacturers (Lyons and Law 2013). In addition, directly comparing vessel sealing data from different studies is not generally advised given the study conditions vary so widely (Lyons and Law 2013). Not only do surgeons have to choose between the sealing technologies available, but they also have to pick between the various device brands. Each of the options can come with varying user experiences, sealing performance, and risks. The difficulties that come with choosing a device due to the range of options 
available emphasizes the need for user experience, reliable knowledge sources, and continued education as the technology in this field rapidly continues to develop.

Several scientific studies indicated the need for a standardized curriculum in the safe use of energy in the operating room (Feldman, Fuchshuber et al. 2012, Watanabe, Kurashima et al. 2016). The Fundamental Safe Use of Energy (FUSE) program was developed to address the training, knowledge, and assessment gaps in the instances where a lack of a standardized curriculum related to surgical energy devices may contribute to a risk for injury (Feldman, Fuchshuber et al. 2012). Feldman et al. distributed surveys to target audiences to identify the areas that required the most focus in the development of the assessment component of the FUSE program (Feldman, Fuchshuber et al. 2012, Feldman, Brunt et al. 2013). Of fifty surveys completed from a target audience that consisted mostly experienced surgeons, only $28 \%$ considered themselves as "experts" in the safe use of the devices that they use regularly (Feldman, Brunt et al. 2013). A separate survey to a target audience that included Society of American Gastrointestinal and Endoscopic Surgeons (SAGES) leaders indicated a knowledge gap in "electrosurgical nomenclature, generator settings, responses to operating room fires, and interactions with other implantable devices." A pretest given as part of a pilot postgraduate course for the FUSE program indicated that surgeons held a minimal understanding of the devices that they use regularly and also train others to use (Feldman, Fuchshuber et al. 2012). Improvement in participant test scores resulted after completion of the pilot FUSE postgraduate course, which demonstrated the need for such a comprehensive curriculum and certification process to educate users about the risks and complications associated with energy use in the operating room. 
One recall involved the Thunderbeat device (Z-0432-2014), and the reason for recall stated "complaints of damage to various models of Thunderbeat hand instruments during surgical procedures, including cracking, breaking, or deformation of the components of the probe tip or jaw." This reason for recall provided by the manufacturer accounted for a quantity in commerce of 8,709 and was directly related to the adverse event outcomes identified in the adverse event reports. Specifically, the recall was consistent with the broken device failures involving the tissue pad and probe tip. The recall was determined by the FDA to be caused by "use error," which is consistent with the possible causes of a broken device described in the adverse event reports. One of the four Harmonic Ace recalls was related to device design (Z-0278-2016), however the specific reason provided by the manufacturer was not consistent with the failure modes identified in the adverse event reports given it involves packaging issues. The following excerpts were taken from manufacturer narratives that described the possible causes for a broken device (FDA 2017):

\section{Manufacturer Narrative - Thunderbeat}

"...the exact cause of the reported event could not be conclusively determined at this time. This type of probe damage is most likely related to the operator's technique. The instruction manual contains several warning statements in an effort to prevent damage to the probe. 'Do not activate output in seal and cut mode while grasping section is closed without contacting tissue or vessel. Do not activate output while applying the probe tip to the tissue with a strong force. Do not activate output while grasping thick and hard tissue. Otherwise, vari[o] us forms of damage in the probe tip and/or the tissue 
pad such as premature wear, breakage, deformation, exposure of metal, and/or falling inside the body cavity, and/or partial separating may occur. '”

\section{Manufacturer Narrative - Harmonic Ace}

“...probable causes of blade damage, including breakage, are external contact during pre-op or general use, blade contact with other devices, staples or clips during the procedure or using any means other than the blade wrench to attach or detach the blade. Once minor blade damage has occurred, subsequent activations may increase damage severity and result in an error code 5 or blade 'lockout' later in the procedure, and continued usage can result in a broken blade..."

Manufacturer Narrative - Harmonic Ace

"The analysis results found that the device was returned with the tissue pad melted and partially detached. Based on the condition of the tissue pad, it appears possible that the clamp of the device may have been closed and the instrument activated without tissue present... Our instruction insert states 'care should be taken not to apply pressure between the instrument blade and tissue pad without having tissue between them. This can result in possible damage to the instrument.",

In these cases, the broken device is suggested as a direct result of improper use. However, based on the context provided within the event descriptions it is not always possible to conclude the root cause of the failure. 


\subsubsection{Manufacturer Narratives and Event Descriptions}

Prevention of user error and device failure requires consistent reporting of adverse events to improve awareness, educational techniques for use, and the development of safe, reliable, user-friendly devices. The FDA MAUDE database is intended for the quick detection and correction of medical device issues, yet various factors need to be improved for the database to be effective as a medical professional and manufacturer resource. It is important to note the differences in terminology provided by the reporting users and facilities for the two types of devices. Failure modes tended to be more explicitly described in the reports associated with endoscopic staplers as compared to tissue sealers. Additionally, the stapler failure terminology was more consistent across the reports and adhered more to the terminology frequently referred to in the scientific literature. In contrast, the tissue sealer reports were more difficult to interpret due to the lack of consistency in failure terminology and overall lack of standardized terminology to clearly describe the cause of failure. The discrepancy may be attributed to the knowledge gap in users' understanding of electrosurgical outcomes overall as mentioned in multiple knowledge gap studies and discussed previously in Section1.2.2.1 (Feldman, Fuchshuber et al. 2012, Feldman, Brunt et al. 2013, Madani, Watanabe et al. 2014, Watanabe, Kurashima et al. 2016). It is not always possible to decipher whether a failure was caused by the user or device malfunction. A standardized curriculum, such as the FUSE program, must be well implemented for physicians and residents in response to technological advancements of surgical instrumentation to fill the knowledge gap and prevent adverse operative outcomes (Chekan, Whelan et al. 2013, Feldman, Brunt et al. 2013, Madani, Watanabe et al. 2014). 
Manufacturers have a responsibility to recognize and address the challenges faced by surgeons during use, and medical device reporting is one source to identify these challenges. The manufacturer narrative included in many adverse event reports is essential to identifying the root cause of failure, which enables manufacturers to implement design changes to prevent further device failure and user errors associated with device operation. Certain component failures may actually be due to user errors such as deploying a stapler on too thick of tissue for the cartridge or reload chosen. Identifying which components failed and the reason for such failure can further heighten a manufacturer's understanding of user error, device failure mechanisms, and the potential association between the two.

By responding to adverse events and providing a manufacture narrative for each reported event, manufacturers are able to reemphasize instructions and warnings while gaining a valuable understanding of the issues users encounter in the operating room. On-going investigation is necessary to characterize the nature and frequency of issues associated with medical devices in order to prevent adverse events and enhance user and manufacturer awareness of potential risks and complications as the technology and procedures continue to advance.

\subsection{Failure Mode Study Limitations and Future Work}

The FDA's MAUDE database is a voluntary database, and it is likely that the number of incidence is underreported. Event descriptions within the reports were not all adequate to allow for phase or failure mode identification in some cases. Furthermore, 
manufacturer narratives were not provided in the Covidien Endo GIA reports, from which valuable insight regarding specific failures can be derived. The accuracy and completeness of the adverse events reports cannot be established or confirmed. It should be noted that in some cases when a failure mode was not explicitly stated, the failure mode was inferred based on the context provided in the event descriptions and manufacture narrative. Therefore, it is possible that misclassification of failure modes could have occurred leading to an improper overall assessment of failure modes.

The overall use associated with surgical stapling devices is unknown as well as the use per device brand. Without quantifying the total usage of devices, the incidence rate of adverse events and corresponding failure modes remains undetermined. The total number of uses of the devices is not recorded and not all complications resulting in adverse events are reported to the FDA MAUDE (Brown and Woo 2004, Overbey, Townsend et al. 2015).

To ensure reports involved a scenario that qualifies as an adverse event, only the events categorized as a death or injury by the reporting facility in the FDA MAUDE database were analyzed. Therefore malfunctions were not accounted for which are associated with additional device failure modes (Riggs, Bohm et al. 2016). From a manufacturer perspective, further investigation that involves malfunction event types could provide valuable insight as it relates to design and a comprehensive device error overview. It is important to note that even though the reports are classified by the event type, the reporting facility classifications are not always accurate. Based on this work and a previous review of endoscopic stapler FDA reports, there is evidence that many reports involving injury have been classified as malfunction and reports that involve 
purely malfunction have been classified as an injury event type (Riggs, Bohm et al. 2016). It is important to be aware of this in future work especially if adverse events that are consistent with the accompanying definition are required (i.e. only injury and death outcomes).

Due to limitations in the allotted resources and budget for obtaining surgical medical devices, only two devices from two device classes were assessed. There are other available brands in the device classes whose adverse event reports should be investigated to provide a more comprehensive assessment. The Thunderbeat is a relatively new device and was limited in the total sample of adverse event reports available in the FDA MAUDE database. On-going investigation into this particular device is suggested as its overall use increases. The endoscopic staplers investigated operate via strictly mechanical mechanisms. Though not covered specifically, powered surgical stapling devices are gaining in popularity, which has also been demonstrated by the increasing appearance of adverse events related to these devices in the MAUDE database as mentioned in a previous review (Riggs, Bohm et al. 2016). In many cases for these devices, the phases of operation in which failure occur would be similar to those identified due to the general features and functional requirements. However, future studies should be conducted observing the specific failure modes of powered surgical stapling devices as their presence in the operating room increases.

This work also aimed to investigate the relationship between the device design complexity and failure mode types associated with endoscopic staplers and energy-based tissue sealers. However, the types of failure modes were not consistent across the two types of minimally invasive surgical devices assessed. Therefore, a statistical method 
could not be employed for this sample provided that the frequencies of each specific failure type could not be grouped in similar categories for all four devices and corresponding complexity scores. Further assessment of devices of the same type would be required in order to investigate this relationship. 


\section{CHAPTER III \\ SEVERITY OF ADVERSE EVENT OUTCOMES}

\section{$\underline{\text { 3.1 Adverse Event Outcome Severity Study Overview }}$}

Identifying and describing failure modes associated with minimally invasive surgical devices is an essential step in improving patient safety. Further, assessing the severity of the adverse outcomes associated with such failures is necessary given the broad range of possible patient consequences. This chapter addresses Specific Aim 2, which was to contribute to provision of a comprehensive evaluation of adverse clinical events by describing the severity of adverse event outcomes that are associated with the failure modes of endoscopic staplers and energy-based tissue sealers. The CTCAE v4.0 grading scale was applied to the adverse event outcomes associated with the failure modes identified in Chapter II.

Adverse events associated with medical device use are concerning due to the potential morbid patient consequences, especially for those that occur during MIS. Recall from Section 1.2.4 that while various studies have broadly focused on assessing the failure modes of medical devices as revealed in FDA MAUDE database reports, none have investigated the severity associated with the failure mode outcomes specific to intraoperative device use. This is the first study to apply an adverse event severity 
grading method to the failure mode outcomes derived from FDA MAUDE database reports associated with intraoperative minimally invasive surgical device use.

Failures during MIS are particularly of concern given the lack of direct accessibility during device use. Issues with device use during MIS increase the possibility of requiring a conversion to an open procedure, procedural delays, prolonged hospital stays, and heightened patient risk. Therefore, it is necessary to not only assess the types of failures, but also the severity associated with the outcomes. The severity assessment supplies users with a better understanding of failures and corresponding adverse event outcomes in the operation setting. It is important to be aware of the types of various failures modes, their relative frequencies, and their associated outcome severity. The work presented is an essential step in the development of an approach for prevention of outcome severities that specifically result in severe, life-threatening, or morbid outcomes on the CTCAE v4.0 grading scale.

\subsection{Adverse Event Outcome Severity Methods}

\subsubsection{Severity Grading}

The severity of user and device-based failure mode outcomes associated with endoscopic staplers and tissue sealers was investigated. The sample of FDA MAUDE reports used in assessing the type and frequency of failure modes (Section 2.2.1.3) was also used for the severity assessment. CTCAE v4.0 grading descriptions were employed in order to assign a severity grade to each adverse event outcome identified through analysis of the event descriptions (NIH 2009). 
In accordance with the CTCAE v4.0 grading outlined in Table 3 in Section 1.2.5.1, a severity of 1 indicates a mild consequence that involves clinical or diagnostic observations only when no intervention is indicated. For example, a minor burn from the residual heat of a tissue sealer would be classified as mild if it did not require any type of intervention, such as a form of a topical treatment. A severity of 2 indicates a moderate event that required local or noninvasive intervention. Following from the previous example, if a burn occurred from residual heat that required topical treatment it would be classified as a moderate outcome of severity 2. In regards to endoscopic staplers, a moderate outcome example would include unanticipated tissue loss (resection) that did not involve tissue damage or further adverse consequences.

An outcome with a severity of 3 is medically significant but not immediately lifethreatening. Severe events can involve prolonged hospitalization or procedural delays. Additionally, severe outcomes relative to these devices are those that involve blood loss (that does not require transfusion), tissue damage, conversion to open surgery, or reoperation due to the adverse event. If a blood transfusion is required it is considered life threatening, which would be a severity of 4 . A death related to the adverse event is a severity of 5 .

All failure modes described within an event description were accounted for, and one severity grade was applied per outcome. Therefore, in the cases where two failure modes were identified in one report's event description, the severity grade of a single outcome was applied to both failure modes for this analysis.

\subsubsection{Adverse Event Outcome Severity Data Analysis}


Severity grade frequencies, proportions, and averages were used to compare severity per phase of operation, failure mode, device brand, and device type. Average outcome severity was calculated for each phase of operation and failure mode. Phase of operation proportions were calculated relative to the total number of failures and corresponding severities for each device type unless indicated otherwise. The percentage of failure outcomes that resulted in a severity of 3 or higher were also reported per phase of operation and device type in order to identify the occurrence of medically significant outcomes. All proportions were rounded to the nearest tenth decimal place. To enable further analysis, common phases of operation across both device types were determined to be transition, main function, and results/post-op. The endoscopic stapler phases of operation utilized in Chapter II were regrouped according to the common phases of operation categories for severity frequencies. Specifically, the reload, application, articulation, and removal phases for endoscopic staplers were grouped into the transition phase, the main function consisted of the firing and cutting phases, and the results/postop phase involved the staple line. The tissue sealer transition, activation, and post-op phases correspond to the transition, main function, and results/post-op common phases, respectively.

Frequencies of each severity grade were determined per common phase of operation and analyzed for association. The cross tabulation table resulted in a cell frequency of less than five; therefore, Fisher's Exact Test was used to indicate association between the common phases of operation and the severity grade of the adverse event outcomes. 


\section{$\underline{3.3 \text { Adverse Event Outcome Severity Results }}$}

\subsubsection{Severity of Failure Mode Outcomes Associated with Endoscopic Staplers}

Failure mode frequencies were grouped using the severity of the associated adverse event outcomes. The severities were averaged per phase of operation and failure mode. The severities associated with the Ethicon ETS endoscopic stapler failure modes are shown in Table 18.

Table 18. Severity of failure mode outcomes associated with the ETS endoscopic stapler ( $\mathrm{n}=111$ failures derived from 100 reports).

\begin{tabular}{|c|c|c|c|c|c|c|c|c|}
\hline \multirow[t]{2}{*}{ Phase } & \multirow{2}{*}{$\begin{array}{l}\text { Average } \\
\text { Severity }\end{array}$} & \multirow[t]{2}{*}{ Failure Mode } & \multicolumn{5}{|c|}{$\begin{array}{c}\text { Outcome Severity } \\
\text { Frequency }\end{array}$} & \multirow{2}{*}{$\begin{array}{l}\text { Average } \\
\text { Severity }\end{array}$} \\
\hline & & & 1 & 2 & 3 & 4 & 5 & \\
\hline \multirow{2}{*}{ Reload } & \multirow{2}{*}{3.60} & Reload fell out of or off of device & & & 4 & 1 & 2 & 3.71 \\
\hline & & Reload malfunction & & & 2 & 1 & & 3.33 \\
\hline Articulation & 3.00 & Articulation malfunction & & & 2 & & & 3.00 \\
\hline Application & 3.00 & Failure to open/close for application on tissue & & & 2 & & & 3.00 \\
\hline \multirow{4}{*}{ Firing } & \multirow{4}{*}{3.27} & Malformed staples/Failure to form staples & & & 15 & 1 & 1 & 3.18 \\
\hline & & Failure to fire (at all/completely/properly) & & 1 & 5 & 3 & & 3.22 \\
\hline & & General misfire (nonspecific) & & 1 & 11 & 2 & & 3.07 \\
\hline & & Failure to deploy staples (at all/on both sides) & & & 11 & 8 & 1 & 3.50 \\
\hline \multirow{2}{*}{ Cutting } & \multirow{2}{*}{2.67} & Unintentionally nicked tissue & & & & & & - \\
\hline & & Failure to cut (at all/completely/properly) & & 1 & 2 & & & 2.67 \\
\hline \multirow{2}{*}{ Removal } & \multirow{2}{*}{2.43} & Failure to release & & 13 & 6 & 1 & & 2.40 \\
\hline & & Difficult release & & & 1 & & & 3.00 \\
\hline \multirow{2}{*}{ Staple line } & \multirow{2}{*}{3.46} & Staple line failure & & & 4 & 2 & & 3.33 \\
\hline & & Staple line leak post-op & & & 4 & 2 & 1 & 3.57 \\
\hline
\end{tabular}

The outcome severities associated with the Covidien Endo GIA endoscopic stapler failure modes are shown in Table 19. 
Table 19. Severity of failure mode outcomes associated with the Endo GIA endoscopic stapler ( $\mathrm{n}=113$ failures derived from 100 reports).

\begin{tabular}{|c|c|c|c|c|c|c|c|c|}
\hline \multirow[t]{2}{*}{ Phase } & \multirow{2}{*}{$\begin{array}{l}\text { Average } \\
\text { Severity }\end{array}$} & \multirow[t]{2}{*}{ Failure Mode } & \multicolumn{5}{|c|}{$\begin{array}{c}\text { Outcome Severity } \\
\text { Frequency }\end{array}$} & \multirow{2}{*}{$\begin{array}{l}\text { Average } \\
\text { Severity }\end{array}$} \\
\hline & & & 1 & 2 & 3 & 4 & 5 & \\
\hline \multirow{2}{*}{ Reload } & \multirow{2}{*}{2.83} & Reload fell out of or off of device & & 1 & 2 & & & 2.67 \\
\hline & & Reload malfunction & & 1 & 1 & 1 & & 3.00 \\
\hline Articulation & - & Articulation malfunction & & & & & & - \\
\hline Application & 3.25 & Failure to open/close for application on tissue & & & 3 & 1 & & 3.25 \\
\hline \multirow{4}{*}{ Firing } & \multirow{4}{*}{2.90} & Malformed staples/Failure to form staples & & 7 & 22 & 1 & & 2.80 \\
\hline & & Failure to fire (at all/completely/properly) & & 5 & 4 & 2 & & 2.73 \\
\hline & & General misfire (nonspecific) & 1 & 1 & 3 & & & 2.40 \\
\hline & & Failure to deploy staples (at all/on both sides) & & 1 & 4 & 7 & & 3.50 \\
\hline \multirow{2}{*}{ Cutting } & \multirow{2}{*}{3.12} & Unintentionally nicked tissue & & & & 1 & 1 & 4.50 \\
\hline & & Failure to cut (at all/completely/properly) & & 2 & 2 & & & 2.50 \\
\hline \multirow{2}{*}{ Removal } & \multirow{2}{*}{2.78} & Failure to release & & 6 & 16 & & & 2.73 \\
\hline & & Difficult release & & & & 1 & & 4.00 \\
\hline \multirow{2}{*}{ Staple line } & \multirow{2}{*}{3.5} & Staple line failure & & & 1 & 4 & 1 & 4.00 \\
\hline & & Staple line leak post-op & & & 8 & 2 & & 3.20 \\
\hline
\end{tabular}

Table 20 includes the total number and proportion of failures that occurred and average outcome severity per the assigned common phase of operation for each endoscopic stapler. The average severities take into account the outcome severity of each failure mode occurrence within each phase of operation.

Table 20. Endoscopic stapler failure frequencies (proportions) and average severity of outcomes per phase.

\begin{tabular}{|c|c|c|c|c|}
\hline $\begin{array}{c}\text { Phase of } \\
\text { Operation }\end{array}$ & $\begin{array}{c}\text { ETS } \\
\text { Failures (\%) }\end{array}$ & $\begin{array}{c}\text { ETS } \\
\text { Average } \\
\text { Severity }\end{array}$ & $\begin{array}{c}\text { Endo GIA } \\
\text { Failures (\%) }\end{array}$ & $\begin{array}{c}\text { Endo GIA } \\
\text { Average } \\
\text { Severity }\end{array}$ \\
\hline Transition & $35(31.5 \%)$ & 2.83 & $33(29.2 \%)$ & 2.85 \\
\hline Main Function & $63(56.8 \%)$ & 3.24 & $64(56.6 \%)$ & 2.92 \\
\hline Result/Post-op & $13(11.7 \%)$ & 3.46 & $16(14.2 \%)$ & 3.50 \\
\hline
\end{tabular}


Endoscopic stapler outcome severity frequencies and proportions relative to the total number of failures assessed are shown in Table 21 per common phase of operation.

Table 21. Endoscopic stapler failure outcome severity frequencies per phase of operation.

\begin{tabular}{|c|c|c|c|c|}
\hline \multirow{2}{*}{ Severity } & \multicolumn{4}{|c|}{ Frequency (\%) } \\
\cline { 2 - 5 } & Transition & Main Function & Results/Post-op & Total \\
\hline $\mathbf{1}$ & - & $1(0.4 \%)$ & - & $1(0.4 \%)$ \\
\hline $\mathbf{2}$ & $21(9.4 \%)$ & $19(8.5 \%)$ & - & $40(17.9 \%)$ \\
\hline $\mathbf{3}$ & $39(17.4 \%)$ & $79(35.3 \%)$ & $17(7.6 \%)$ & $135(60.3 \%)$ \\
\hline $\mathbf{4}$ & $6(2.7 \%)$ & $25(11.2 \%)$ & $10(4.5 \%)$ & $41(18.3 \%)$ \\
\hline $\mathbf{5}$ & $2(0.9 \%)$ & $3(1.3 \%)$ & $2(0.9 \%)$ & $7(3.1 \%)$ \\
\hline Total & $68(30.4 \%)$ & $127(56.7 \%)$ & $29(12.9 \%)$ & $224(100 \%)$ \\
\hline
\end{tabular}

The average severity of adverse event outcomes associated with each common phase of operation for endoscopic staplers is shown per phase in Table 22 in addition to the overall percentage of failure outcomes that resulted in a severity of 3 or higher. The percentages are relative to the total number of outcomes across all phases of operation.

Table 22. Endoscopic stapler average severity per phase of operation and overall percentage of failure outcomes with severity of 3 or higher.

\begin{tabular}{|c|c|c|}
\hline Phase of Operation & Average Severity & \% with Severity $\geq \mathbf{3}$ \\
\hline Transition & 2.84 & $21.0 \%$ \\
\hline Main Function & 3.08 & $47.8 \%$ \\
\hline Result/Post-op & 3.48 & $12.9 \%$ \\
\hline
\end{tabular}

Table 23 provides the percentage of outcomes that resulted in a severity of 3 or higher relative to the number of outcomes per common phase of operation. 
Table 23. Endoscopic stapler percentage of outcomes with severity 3 or higher relative to the number of outcomes that occurred during each phase of operation.

\begin{tabular}{|c|c|}
\hline Phase of Operation & \% with Severity $\geq \mathbf{3}$ \\
\hline Transition & $69.1 \%$ \\
\hline Main Function & $84.3 \%$ \\
\hline Result/Post-op & $100 \%$ \\
\hline
\end{tabular}

The distribution of the severity outcome data per common phase of operation is shown in the boxplot in Figure 6.

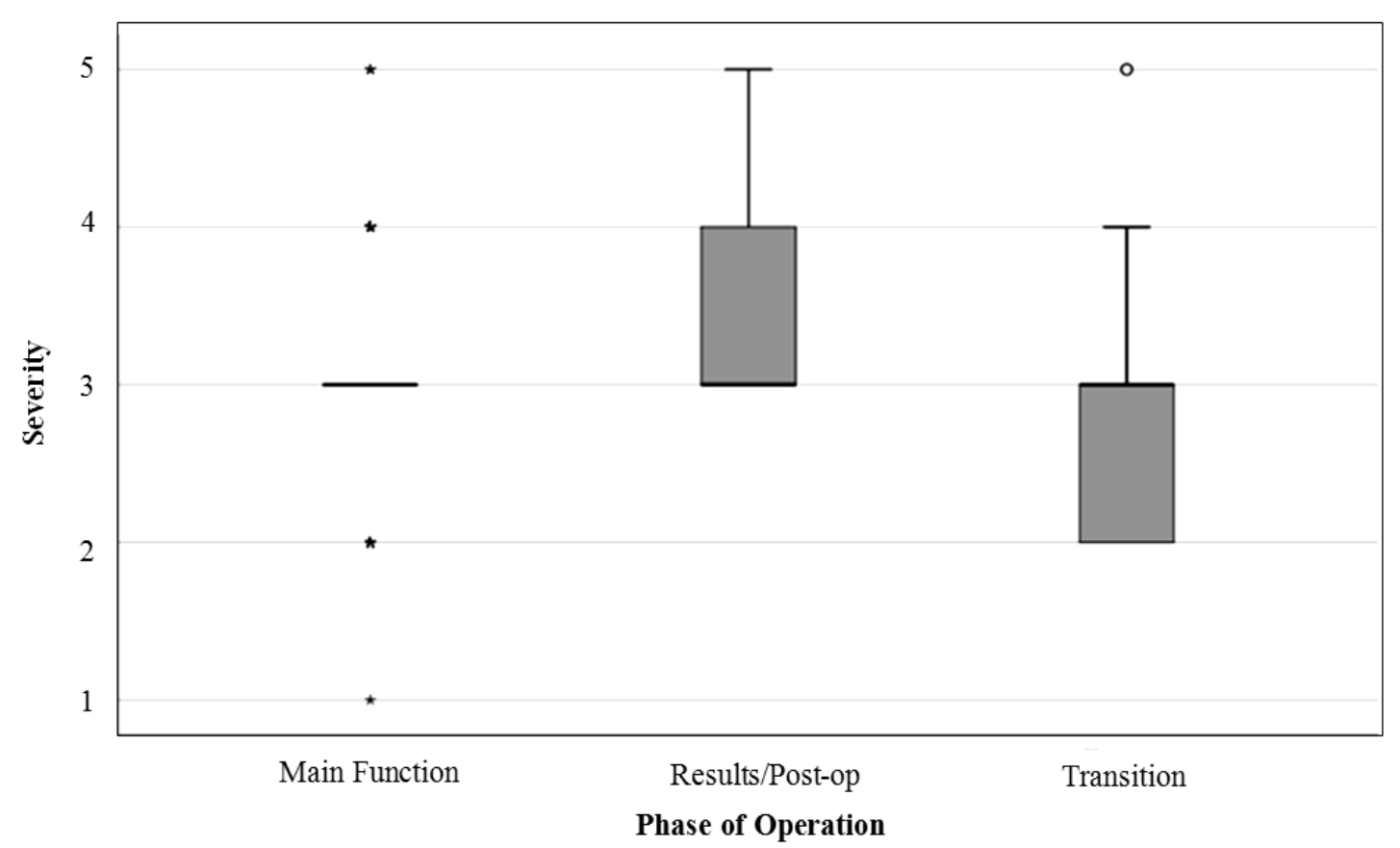

Figure 6. Distribution of severity per phase of operation associated with endoscopic staplers.

Fisher's Exact Test was conducted in order to determine the association between the severity of adverse event outcomes and the phases of operation. The cross tabulation 
table (Table 24) shows the frequency counts and column percentages, and Fisher's Exact Test results are shown in Table 25 .

Table 24. Endoscopic stapler cross tabulation table.

\begin{tabular}{|c|c|c|c|c|c|c|}
\hline \multirow{2}{*}{ Phase } & \multicolumn{6}{|c|}{ Severity Count (\%) } \\
\cline { 2 - 7 } & $\mathbf{1}$ & $\mathbf{2}$ & $\mathbf{3}$ & $\mathbf{4}$ & $\mathbf{5}$ & Total \\
\hline Main Function & $1(100 \%)$ & $19(47.5 \%)$ & $79(58.5 \%)$ & $25(61.0 \%)$ & $3(42.9 \%)$ & $127(56.7 \%)$ \\
\hline Results/Post-op & $0(0.0 \%)$ & $0(0.0 \%)$ & $17(12.6 \%)$ & $10(24.4 \%)$ & $2(28.6 \%)$ & $29(12.9 \%)$ \\
\hline Transition & $0(0.0 \%)$ & $21(52.5 \%)$ & $39(28.9 \%)$ & $6(14.6 \%)$ & $2(28.6 \%)$ & $68(30.4 \%)$ \\
\hline Total & $1(100 \%)$ & $40(100 \%)$ & $135(100 \%)$ & $41(100 \%)$ & $7(100 \%)$ & $224(100 \%)$ \\
\hline
\end{tabular}

Table 25. Chi-Squared tests SPSS output for endoscopic stapler outcome severity and phase of operation.

\begin{tabular}{|c|c|c|c|c|c|c|}
\hline & \multirow[b]{3}{*}{ Value } & \multirow{3}{*}{$\begin{array}{l}\text { Degrees } \\
\text { of } \\
\text { Freedom }\end{array}$} & \multirow{3}{*}{$\begin{array}{c}\text { Asymptotic } \\
\text { Significance } \\
\text { (2-sided) }\end{array}$} & \multicolumn{3}{|c|}{ Monte Carlo Sig. (2-sided) } \\
\hline & & & & \multirow[b]{2}{*}{ P-value } & \multicolumn{2}{|c|}{$\begin{array}{c}95 \% \text { Confidence } \\
\text { Interval }\end{array}$} \\
\hline & & & & & $\begin{array}{l}\text { Lower } \\
\text { Bound }\end{array}$ & $\begin{array}{l}\text { Upper } \\
\text { Bound }\end{array}$ \\
\hline Pearson Chi-Square & $22.370^{\mathrm{a}}$ & 8 & 0.004 & $<0.01^{\mathrm{b}}$ & 0.000 & 0.020 \\
\hline Likelihood Ratio & 26.399 & 8 & 0.001 & $<0.01^{\mathrm{b}}$ & 0.000 & 0.020 \\
\hline Fisher's Exact Test & 24.061 & & & $<0.01^{\mathrm{b}}$ & 0.000 & 0.020 \\
\hline N of Valid Cases & 224 & & & & & \\
\hline
\end{tabular}

a. 6 cells (40.0\%) have expected count less than 5. The minimum expected count 0.13 .

b. Based on 224 sampled tables with starting seed 1314643744.

The Fisher's Exact Test significance value indicates there was an association between the severity of the outcome and the phase in which the adverse event occurred ( $p$ $<0.05)$. The severity of the outcomes in the various phases of endoscopic stapler operation was significantly different.

3.3.2 Severity of Failure Mode Outcomes Associated with Tissue Sealers 
Failure mode frequencies were grouped using the severity of the associated adverse event outcomes. The severities were averaged per phase of operation and failure mode. The outcome severities associated with the Thunderbeat device failure modes are shown in Table 26.

Table 26. Severity of failure modes associated with the Thunderbeat tissue sealer ( $n=81$ failures derived from 83 reports).

\begin{tabular}{|c|c|c|c|c|c|c|c|c|}
\hline \multirow[t]{2}{*}{ Phase } & \multirow{2}{*}{$\begin{array}{l}\text { Average } \\
\text { Severity }\end{array}$} & \multirow[t]{2}{*}{ Failure Mode } & \multicolumn{5}{|c|}{$\begin{array}{c}\text { Outcome Severity } \\
\text { Frequency }\end{array}$} & \multirow{2}{*}{$\begin{array}{l}\text { Average } \\
\text { Severity }\end{array}$} \\
\hline & & & 1 & 2 & 3 & 4 & 5 & \\
\hline \multirow{7}{*}{ Activation } & \multirow{7}{*}{2.36} & Unspecific error resulting in bleeding & & & & & 1 & 5.00 \\
\hline & & Broken device - blade or probe & 6 & 5 & & & & 1.45 \\
\hline & & Broken device - tissue pad & 2 & 2 & 3 & & & 2.14 \\
\hline & & Failure to seal/failure to seal properly & 2 & & 4 & & & 2.33 \\
\hline & & Direct application & & 1 & 4 & 1 & 1 & 3.29 \\
\hline & & Thermal spread & & & 2 & & & 3.00 \\
\hline & & Insulation Failure & & & 2 & & & 3.00 \\
\hline \multirow{5}{*}{ Transition } & \multirow{5}{*}{2.37} & Residual heat & 1 & 12 & 10 & & & 2.39 \\
\hline & & Broken device - blade or probe & 1 & & & & & 1.00 \\
\hline & & Broken device - tissue pad & & & & & & - \\
\hline & & Inadvertent application & & 1 & 4 & & & 2.80 \\
\hline & & Installation error; electric shock & 1 & & & & & 1.00 \\
\hline \multirow{2}{*}{ Post-op } & \multirow{2}{*}{3.53} & Seal failure with bleeding/leakage & & & 11 & & 4 & 3.53 \\
\hline & & Tissue injury detected after device use & & & & & & - \\
\hline
\end{tabular}

The outcome severities associated with the Harmonic device failure modes are shown in Table 27. 
Table 27. Severity of failure mode outcomes associated with the Harmonic Ace tissue sealer ( $n=102$ failures derived from 100 reports).

\begin{tabular}{|c|c|c|c|c|c|c|c|c|}
\hline \multirow[t]{2}{*}{ Phase } & \multirow{2}{*}{$\begin{array}{l}\text { Average } \\
\text { Severity }\end{array}$} & \multirow[t]{2}{*}{ Failure Mode } & \multicolumn{5}{|c|}{$\begin{array}{c}\text { Outcome Severity } \\
\text { Frequency }\end{array}$} & \multirow{2}{*}{$\begin{array}{l}\text { Average } \\
\text { Severity }\end{array}$} \\
\hline & & & 1 & 2 & 3 & 4 & 5 & \\
\hline \multirow{7}{*}{ Activation } & \multirow{7}{*}{2.40} & Unspecific error resulting in bleeding & & & 2 & 1 & 1 & 3.75 \\
\hline & & Broken device - blade or probe & 1 & 3 & 6 & & & 2.50 \\
\hline & & Broken device - tissue pad & 12 & & 2 & & & 1.29 \\
\hline & & Failure to seal/failure to seal properly & 1 & 3 & 10 & 2 & & 2.81 \\
\hline & & Direct application & & & 2 & 1 & & 3.33 \\
\hline & & Thermal spread & & & & & & - \\
\hline & & Insulation Failure & & & & & & - \\
\hline \multirow{5}{*}{ Transition } & \multirow{5}{*}{2.08} & Residual heat & 3 & 5 & 7 & & 1 & 2.44 \\
\hline & & Broken device - blade or probe & 2 & 1 & & & & 1.33 \\
\hline & & Broken device - tissue pad & 4 & & & & & 1.00 \\
\hline & & Inadvertent application & & & 1 & & & 3.00 \\
\hline & & Installation error; electric shock & & & & & & - \\
\hline \multirow{2}{*}{ Post-op } & \multirow{2}{*}{3.52} & Seal failure with bleeding/leakage & & 1 & 12 & 15 & 1 & 3.55 \\
\hline & & Tissue injury detected after device use & & & 2 & & & 3.00 \\
\hline
\end{tabular}

Two adverse event reports associated with the Thunderbeat device did not indicate a specific failure mode; however, it was indicated that the failures occurred postop and resulted in death (severity of 5). These two reports are included in Table 28 which lists the total number and proportion of failures that occurred and average outcome severity per the assigned common phase of operation for each tissue sealer.

Table 28. Tissue sealer failure frequencies (proportions) and average outcome severity per phase.

\begin{tabular}{|c|c|c|c|c|}
\hline $\begin{array}{c}\text { Phase of } \\
\text { Operation }\end{array}$ & $\begin{array}{c}\text { Thunderbeat } \\
\text { Failures (\%) }\end{array}$ & $\begin{array}{c}\text { Thunderbeat } \\
\text { Average } \\
\text { Severity }\end{array}$ & $\begin{array}{c}\text { Harmonic } \\
\text { Failures (\%) }\end{array}$ & $\begin{array}{c}\text { Harmonic } \\
\text { Average } \\
\text { Severity }\end{array}$ \\
\hline Transition & $30(36.1 \%)$ & 2.37 & $24(23.5 \%)$ & 2.08 \\
\hline Main Function & $36(43.4 \%)$ & 2.36 & $47(46.1 \%)$ & 2.40 \\
\hline Result/Post-op & $17(20.5 \%)$ & 3.70 & $31(30.4 \%)$ & 3.52 \\
\hline
\end{tabular}


Tissue sealer failure outcome severity frequencies and proportions relative to the total number of failures assessed are shown in Table 29 per common phase of operation.

Table 29. Tissue sealer failure outcome severity frequencies per phase of operation.

\begin{tabular}{|c|c|c|c|c|}
\hline \multirow{2}{*}{ Severity } & \multicolumn{4}{|c|}{ Frequency (\%) } \\
\cline { 2 - 5 } & Transition & Main Function & Results/Post-op & Total \\
\hline $\mathbf{1}$ & $12(6.5 \%)$ & $24(13.0 \%)$ & - & $36(19.5 \%)$ \\
\hline $\mathbf{2}$ & $19(10.3 \%)$ & $14(7.6 \%)$ & $1(0.5 \%)$ & $34(18.4 \%)$ \\
\hline $\mathbf{3}$ & $22(11.9 \%)$ & $37(20.0 \%)$ & $25(13.5 \%)$ & $84(45.4 \%)$ \\
\hline $\mathbf{4}$ & - & $5(2.7 \%)$ & $15(8.1 \%)$ & $20(10.8 \%)$ \\
\hline $\mathbf{5}$ & $1(0.5 \%)$ & $3(1.6 \%)$ & $7(3.8 \%)$ & $11(5.9 \%)$ \\
\hline Total & $54(29.2 \%)$ & $83(44.9 \%)$ & $48(25.9 \%)$ & $185(100 \%)$ \\
\hline
\end{tabular}

The severity of adverse event outcomes associated with each common phase of operation for tissue sealers is shown per phase in Table 30 in addition to the overall percentage of failure outcomes that resulted in a severity of 3 or higher. The percentages are relative to the total number of outcomes across all phases of operation.

Table 30. Tissue sealer average severity per phase of operation and overall percentage of failure outcomes with severity of 3 or higher.

\begin{tabular}{|c|c|c|}
\hline Phase of Operation & Average Severity & \% with Severity $\geq \mathbf{3}$ \\
\hline Transition & 2.24 & $12.4 \%$ \\
\hline Main Function & 2.39 & $24.3 \%$ \\
\hline Result/Post-op & 3.58 & $25.4 \%$ \\
\hline
\end{tabular}

Table 31 shows the percentage of outcomes that resulted in a severity of 3 or higher relative to the number of outcomes per phase of operation. 
Table 31. Tissue sealer percentage of outcomes with severity 3 or higher relative to the number of outcomes that occurred during each phase of operation.

\begin{tabular}{|c|c|}
\hline Phase of Operation & \% with Severity $\geq \mathbf{3}$ \\
\hline Transition & $42.6 \%$ \\
\hline Main Function & $54.2 \%$ \\
\hline Result/Post-op & $97.9 \%$ \\
\hline
\end{tabular}

The distribution of severity outcome data per common phase of operation is shown in the boxplot in Figure 7.

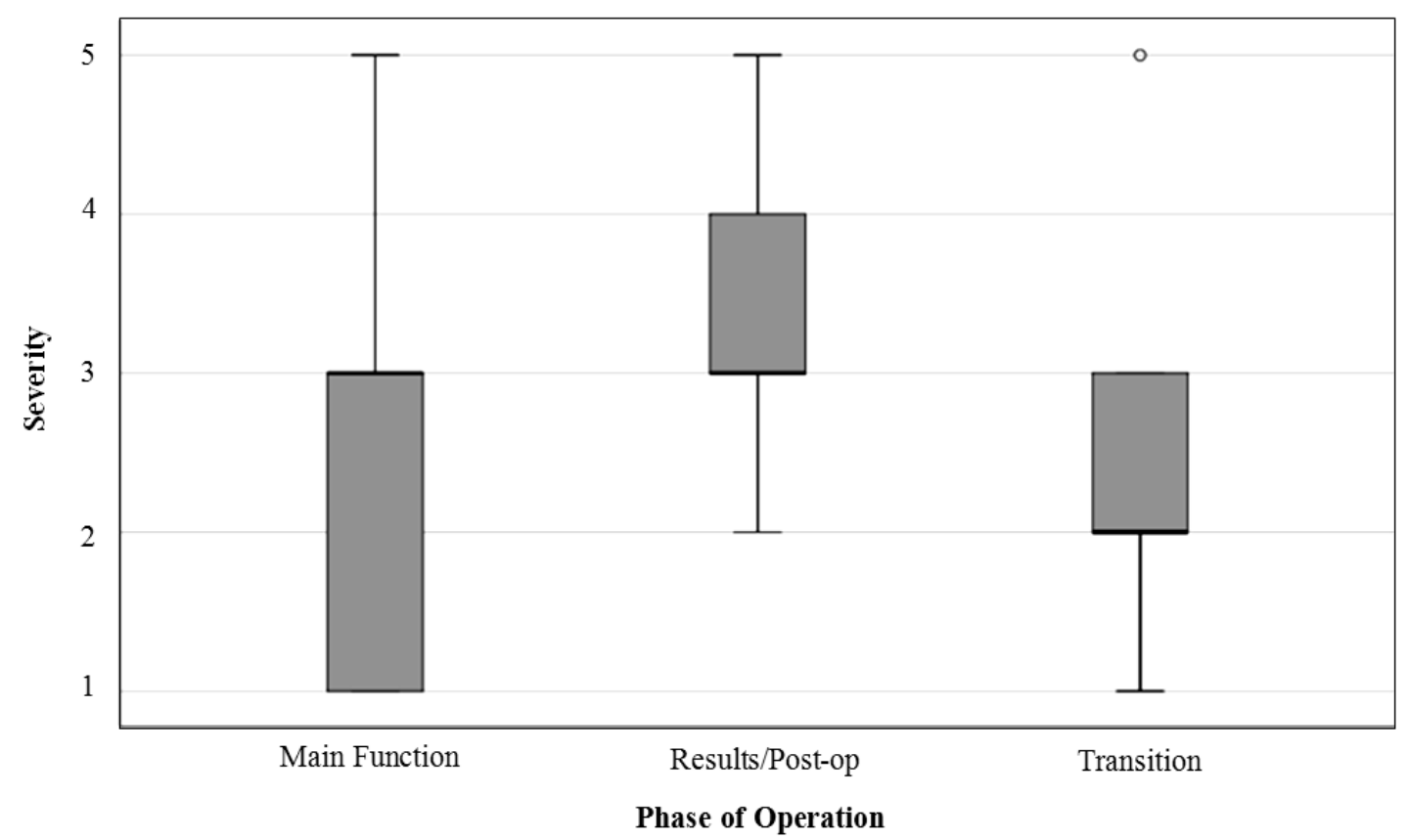

Figure 7. Distribution of severity per phase of operation associated with tissue sealers.

Fisher's Exact Test was conducted in order to determine the association between the severity of adverse event outcomes and the common phases of operation. The cross tabulation table (Table 32) shows the frequency counts and column percentages, and Fisher's Exact Test results are shown in Table 33. 
Table 32. Tissue sealer cross tabulation table.

\begin{tabular}{|c|c|c|c|c|c|c|}
\hline \multirow{2}{*}{ Phase } & \multicolumn{6}{|c|}{ Severity Count (\%) } \\
\cline { 2 - 7 } & $\mathbf{1}$ & $\mathbf{2}$ & $\mathbf{3}$ & $\mathbf{4}$ & $\mathbf{5}$ & Total \\
\hline Main Function & $24(66.7 \%)$ & $14(41.2 \%)$ & $37(44.0 \%)$ & $5(25 \%)$ & $3(27.3 \%)$ & $83(44.9 \%)$ \\
\hline Results/Post-op & $0(0.0 \%)$ & $1(2.9 \%)$ & $25(29.8 \%)$ & $15(75 \%)$ & $7(63.6 \%)$ & $48(25.9 \%)$ \\
\hline Transition & $12(33.3 \%)$ & $19(55.9 \%)$ & $22(26.2 \%)$ & $0(0.0 \%)$ & $1(0.1 \%)$ & $54(29.2 \%)$ \\
\hline Total & $36(100 \%)$ & $34(100 \%)$ & $84(100 \%)$ & $20(100 \%)$ & $11(100 \%)$ & $185(100 \%)$ \\
\hline
\end{tabular}

Table 33. Chi-Squared tests SPSS output for tissue sealer outcome severity and phase of operation.

\begin{tabular}{|c|c|c|c|c|c|c|}
\hline & \multirow[b]{3}{*}{ Value } & \multirow{3}{*}{$\begin{array}{l}\text { Degrees } \\
\text { of } \\
\text { Freedom }\end{array}$} & \multirow{3}{*}{$\begin{array}{l}\text { Asymptotic } \\
\text { Significance } \\
\text { (2-sided) }\end{array}$} & \multicolumn{3}{|c|}{ Monte Carlo Sig. (2-sided) } \\
\hline & & & & \multirow[b]{2}{*}{$\mathbf{P}$-value } & \multicolumn{2}{|c|}{$\begin{array}{l}\text { 95\% Confidence } \\
\text { Interval }\end{array}$} \\
\hline & & & & & $\begin{array}{l}\text { Lower } \\
\text { Bound }\end{array}$ & $\begin{array}{l}\text { Upper } \\
\text { Bound }\end{array}$ \\
\hline Pearson Chi-Square & $63.896^{\mathrm{c}}$ & 8 & 0.000 & $<0.01^{\mathrm{d}}$ & 0.000 & 0.025 \\
\hline Likelihood Ratio & 74.087 & 8 & 0.000 & $<0.01^{\mathrm{d}}$ & 0.000 & 0.025 \\
\hline Fisher's Exact Test & 65.513 & & & $<0.01^{\mathrm{d}}$ & 0.000 & 0.025 \\
\hline $\mathrm{N}$ of Valid Cases & 185 & & & & & \\
\hline
\end{tabular}

c. 3 cells $(6.7 \%)$ have expected count less than 5 . The minimum expected count 2.85

d. Based on 185 sampled tables with starting seed 2000000.

The Fisher's Exact Test significance value indicates there was an association between the severity of the outcome and the phase in which the adverse event occurred ( $p$ $<0.05)$. The severity of the outcomes in the various phases of tissue sealer operation was significantly different.

\subsubsection{Severity of Failure Mode Outcomes Associated with Both Device Types}

The failure outcome severities associated with both device types were combined then grouped per common phase of operation. The combined outcome severity 
frequencies and proportions relative to the total number of failures assessed are shown in Table 34 per phase of operation.

Table 34. Combined failure outcome severity frequencies and proportions.

\begin{tabular}{|c|c|c|c|c|}
\hline \multirow{2}{*}{ Severity } & \multicolumn{4}{|c|}{ Frequency (\%) } \\
\cline { 2 - 5 } & Transition & Main Function & Results/Post-op & Total \\
\hline $\mathbf{1}$ & $12(2.9 \%)$ & $25(6.1 \%)$ & - & $37(9.0 \%)$ \\
\hline $\mathbf{2}$ & $40(9.8 \%)$ & $33(8.1 \%)$ & $1(0.2 \%)$ & $74(18.1 \%)$ \\
\hline $\mathbf{3}$ & $61(14.9 \%)$ & $116(28.4 \%)$ & $42(10.3 \%)$ & $219(53.5 \%)$ \\
\hline $\mathbf{4}$ & $6(1.5 \%)$ & $30(7.3 \%)$ & $25(6.1 \%)$ & $61(14.9 \%)$ \\
\hline $\mathbf{5}$ & $3(0.7 \%)$ & $6(1.5 \%)$ & $9(2.2 \%)$ & $18(4.4 \%)$ \\
\hline Total & $122(29.8 \%)$ & $210(51.3 \%)$ & $77(18.8 \%)$ & $409(100 \%)$ \\
\hline
\end{tabular}

The severity of adverse event outcomes associated with each common phase of operation for the surgical devices is shown per phase in Table 35 in addition to the overall percentage of failure outcomes that resulted in a severity of 3 or higher. The percentages are relative to the total number of outcomes across all phases of operation.

Table 35. Surgical device average severity per phase of operation and overall percentage of failure outcomes with severity of 3 or higher.

\begin{tabular}{|c|c|c|}
\hline Phase of Operation & Average Severity & \% with Severity $\geq \mathbf{3}$ \\
\hline Transition & 2.57 & $17.1 \%$ \\
\hline Main Function & 2.80 & $37.2 \%$ \\
\hline Result/Post-op & 3.55 & $18.6 \%$ \\
\hline
\end{tabular}

Table 36 provides the percentage of outcomes that resulted in a severity of 3 or higher relative to the number of outcomes per phase. 
Table 36. Surgical device percentage of outcomes with severity 3 or higher relative to the number of outcomes that occurred during each phase of operation.

\begin{tabular}{|c|c|}
\hline Phase of Operation & \% with Severity $\geq \mathbf{3}$ \\
\hline Transition & $57.4 \%$ \\
\hline Main Function & $72.4 \%$ \\
\hline Result/Post-op & $98.7 \%$ \\
\hline
\end{tabular}

The percentage of failure outcomes that resulted in a severity of 3 or higher relative to the total number of outcomes per device type is shown in Table 37.

Table 37. Overall percentage of failure outcomes with severity of 3 or higher relative to the number of outcomes that occurred during each phase of operation.

\begin{tabular}{|c|c|}
\hline Device Type & \% with Severity $\geq \mathbf{3}$ \\
\hline Endoscopic Staplers & $81.7 \%$ \\
\hline Tissue Sealers & $62.2 \%$ \\
\hline Both Device Types & $72.8 \%$ \\
\hline
\end{tabular}

The distribution of the combined severity outcome data per common phase of operation is shown in the boxplot in Figure 8. 


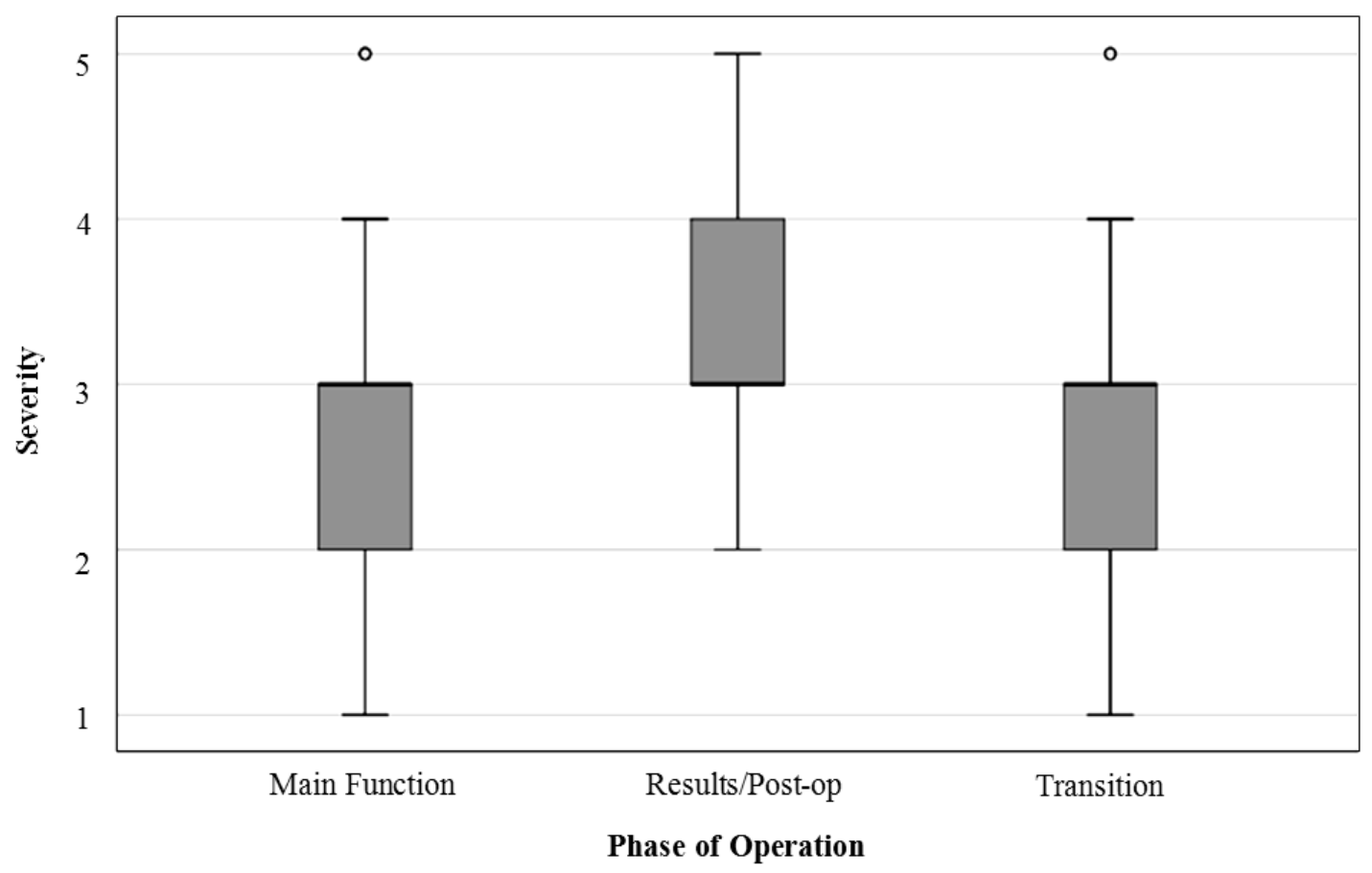

Figure 8. Distribution of severity per phase of operation.

Fisher's Exact Test was conducted in order to determine the association between the severity of adverse event outcomes and the phases of surgical device operation. The cross tabulation table (Table 38) shows the frequency counts and column percentages, and Fisher's Exact Test results are shown in Table 39.

Table 38. Surgical device cross tabulation table.

\begin{tabular}{|c|c|c|c|c|c|c|}
\hline \multirow{2}{*}{ Phase } & \multicolumn{7}{|c|}{ Severity Count (\%) } \\
\cline { 2 - 7 } & $\mathbf{1}$ & $\mathbf{2}$ & $\mathbf{3}$ & $\mathbf{4}$ & $\mathbf{5}$ & Total \\
\hline Main Function & $25(67.6 \%)$ & $33(44.6 \%)$ & $116(53.0 \%)$ & $30(49.2 \%)$ & $6(33.3 \%)$ & $210(51.3 \%)$ \\
\hline Results/Post-op & $0(0.0 \%)$ & $1(1.4 \%)$ & $42(19.2 \%)$ & $25(41.0 \%)$ & $9(50 \%)$ & $77(18.8 \%)$ \\
\hline Transition & $12(32.4 \%)$ & $40(54.1 \%)$ & $61(27.9 \%)$ & $6(9.8 \%)$ & $3(16.7 \%)$ & $122(29.8 \%)$ \\
\hline Total & $37(100 \%)$ & $74(100 \%)$ & $219(100 \%)$ & $61(100 \%)$ & $18(100 \%)$ & $409(100 \%)$ \\
\hline
\end{tabular}


Table 39. Chi-Squared tests SPSS output for surgical device outcome severity and phase of operation.

\begin{tabular}{|c|c|c|c|c|c|c|}
\hline & \multirow[b]{3}{*}{ Value } & \multirow{3}{*}{$\begin{array}{l}\text { Degrees } \\
\text { of } \\
\text { Freedom }\end{array}$} & \multirow{3}{*}{$\begin{array}{c}\text { Asymptotic } \\
\text { Significance } \\
\text { (2-sided) }\end{array}$} & \multicolumn{3}{|c|}{ Monte Carlo Sig. (2-sided) } \\
\hline & & & & \multirow[b]{2}{*}{ P-value } & \multicolumn{2}{|c|}{$\begin{array}{c}\text { 95\% Confidence } \\
\text { Interval } \\
\end{array}$} \\
\hline & & & & & $\begin{array}{l}\text { Lower } \\
\text { Bound }\end{array}$ & $\begin{array}{l}\text { Upper } \\
\text { Bound }\end{array}$ \\
\hline Pearson Chi-Square & $72.189^{\mathrm{e}}$ & 8 & 0.000 & $<0.01^{\mathrm{f}}$ & 0.000 & 0.011 \\
\hline Likelihood Ratio & 81.048 & 8 & 0.000 & $<0.01^{\mathrm{f}}$ & 0.000 & 0.011 \\
\hline Fisher's Exact Test & 75.335 & & & $<0.01^{\mathrm{f}}$ & 0.000 & 0.011 \\
\hline N of Valid Cases & 409 & & & & & \\
\hline
\end{tabular}

e. 1 cell $(6.7 \%)$ has expected count less than 5. The minimum expected count is 3.39.

f. Based on 409 sampled tables with starting seed 1502173562.

The Fisher's Exact Test significance value indicates there was an association between the severity of the outcome and the phase in which the adverse event occurred ( $p$ $<0.05)$. Overall, the severity of the outcomes was significantly different per the various phases of surgical device operation.

\section{$\underline{3.4 \text { Adverse Event Outcome Severity Discussion }}$}

The objective of Specific Aim 2 was to describe the severity of adverse event outcomes that are associated with the failure modes of endoscopic staplers and energybased tissue sealers. The average outcome severity was determined for each failure mode and phase of operation that was identified in Chapter II. The percentage of failure outcomes that resulted in a severity of 3 or higher was reported in order to assess the occurrence of medically significant adverse outcomes. Severity frequencies and proportions were determined per phase of operation for the devices. The frequencies of each severity grade were analyzed for association with the common phases of operation. 


\subsubsection{Endoscopic Stapler Adverse Event Outcome Severity}

The Ethicon ETS stapler failure modes associated with the most severe outcomes involved the reload falling out of or off of the device (average severity of 3.71), staple line leak post-op (3.57), and the failure to deploy staples at all or on both sides (3.50). Only two failure modes resulted in an average outcome severity of less than 3 , which were the failure to release (2.40) and the failure to cut (2.67). These results do not reflect the frequency of each failure mode. Recall from Section 2.3.2, the most common failure modes associated with the ETS stapler were the failure to deploy staples at all or on both sides $(18 \%)$, failure to release (18\%), and malformed staples or failure to form staples (15.3\%). The failure to deploy staples at all or on both sides was a common failure mode for this device and resulted in one of the highest average outcome severities. The failure to release resulted in the lowest average outcome severity associated with the ETS stapler of 2.40. Malformed staples or failure to form staples resulted in an average outcome severity of 3.18. The reload falling out of or off the device and staple line leak post-op each accounted for $6.3 \%$ of the failures.

The Covidien Endo GIA failure modes associated with the most severe outcomes were the unintentional nicking of tissue (4.50), difficult release (4.00), and staple line failure (4.00). Though, these failure modes accounted for a relatively low number of failures associated with the Endo GIA stapler as reported in Section 2.3.2.

Unintentionally nicking tissue accounted for $1.8 \%$ of failures, a difficult release accounted for $0.9 \%$, and staple line failure accounted for $5.3 \%$. The least severe outcomes associated with the Endo GIA stapler resulted from a general misfire (nonspecific) (2.40), the failure to cut (2.50), and the reload falling out of or off the 
device (2.67). The most common failure modes associated with the Endo GIA stapler were malformed staples or failure to form staples (26.5\%), the failure to release $(19.5 \%)$, and failure to deploy staples at all or on both sides (10.6\%); these failure modes resulted in average outcome severities of $2.80,2.73$, and 3.50 , respectively.

The failure to deploy staples at all or on both sides was a common failure mode for both the Ethicon ETS and the Covidien Endo GIA. The average outcome severity was the same for both devices (3.50). The average outcome severity falls between severe and life-threatening on the CTCAE v4.0 grading scale. Therefore, considering the relative frequency and average outcome severity associated with this failure mode across both staplers, the failure to deploy staples at all or on both sides is noteworthy. Given the nature of this failure mode, it is concerning that it occurred relatively frequently since the failure to deploy staples at all or both sides leaves the tissue susceptible to being cut without being simultaneously secured with staples.

Cutting without applying staples accounted for $10.7 \%$ of the 84 adverse event reports retrieved from the FDA MAUDE database by Kwazneski et al. (Kwazneski, Six et al. 2012). The 84 adverse event reports were retrieved using the keyword "misfire" and searching for both Ethicon and Covidien laparoscopic stapling devices (Kwazneski, Six et al. 2012). The failure mode identified in the scientific literature is similar to the one herein except that it does not include failure to deploy on both sides of tissue. A concerning consequence takes place when the knife is deployed yet only one side of staples is deployed, especially when staples are not deployed on the patient side of the tissue. For this reason, there are preventative techniques that have been suggested for laparoscopic stapling given the potentially high outcome severity of this failure. For 
example, Deng et al. discuss a modification of a vascular ligation technique which first utilizes a device that only deploys staples, then proceeds to divide tissue only after it is secured (Deng, Meng et al. 2002). Clamping or applying clips to tissue and structures that are prone to bleeding and leakage prior to deploying a stapling device may prevent or reduce the severity of an adverse event if a failure during firing occurs. However, care should be taken when using any type of clip near the stapling site since contact with such an obstruction can disrupt the firing sequence of an endoscopic stapler (Mansour, ElNashar et al. 2014, Riggs, Bohm et al. 2016).

Malformed staples or the failure to form staples was a common failure for both staplers, but resulted in a relatively lower outcome severity (ETS -3.18 , Endo GIA 2.80). Despite being low relative to other outcome severity averages, these average outcome severities are deemed severe and medically significant (severity grade of 3 ) if rounded to the nearest severity grade. The failure mode involves staples that are deployed from the device but are either not formed correctly or not formed at all. Malformed staples are noted consistently as a concern in scientific literature and can be caused by a number of staple leg bending factors (Deng, Meng et al. 2002, Chekan and Whelan 2014, Fuller, Paull et al. 2014). Examples of factors that affect staple leg bending include staple height, type of metal (bending characteristics), and tissue properties (thickness, viscosity, etc.) (Mery, Shafi et al. 2008, Chekan and Whelan 2014). Chekan and Whelan provide examples of acceptable and unacceptable staple forms produced after firing staples into tissue (Chekan and Whelan 2014). Malformed staples can result in failure of the staple line, tissue trauma, bleeding, or leakage immediately after firing (Mery, Shafi et al. 2008). If malformed staples are not noticed and addressed promptly by the surgical 
team, it can potentially cause detrimental consequences later in the surgery or post-

operatively. Specifically, Chekan and Whelan state the unacceptable staple forms can "compromise integrity and strength of the staple line resulting in an increase rate of leaks and bleeding" (Chekan and Whelan 2014).

Device failure to release from tissue was another common failure mode for both staplers that resulted in a relatively low outcome severity (ETS -2.40 , Endo GIA -2.73 ). The average outcome severities fall between a moderate (2) and severe (3) outcome. The severity of these outcomes is likely dependent on the type of tissue that was fired upon and the procedure type. When a device fails to release from tissue during a minimally invasive procedure the user typically must create another port and apply another stapling device in order to remove the malfunctioning device, which involves additional tissue resection (Kwazneski, Six et al. 2012, Riggs, Bohm et al. 2016). Depending on the tissue type and procedural setup, additional tissue resection may or may not be an acceptable option. Conversion to an open surgery is necessary in such cases and if visibility is hindered or the creation of another port is not an available option.

The ETS stapler was associated with more reload failures than the Covidien Endo GIA (ETS - 9\%, Endo GIA - 5.3\%). Further, the instances where the reload fell completely out of the ETS stapler resulted in more severe outcomes on average than that of the Covidien Endo GIA (ETS - 3.71, Endo GIA - 2.67). This failure mode resulted in the highest average outcome severity of all failure modes associated with the ETS stapler, which is likely due to the nature of the outcome. Adverse event outcomes are likely severe since the consequence of this failure involves the firing of a stapler on tissue without inserting staples (Champion and Williams 2003). The user experiences reported 
in the FDA MAUDE database have noted detrimental consequences as a result of this error, such as life-threatening blood loss (FDA 2017). This failure mode could be due to the reload insertion process dictated by the very design of the device jaw. This event can occur if the reload is not inserted properly or if it "pops" out of place prior to the firing. Further developments of this device have incorporated safety features to prevent the ability to fire without a reload present, but there is evidence that these prevention measures can be overridden and the surgeon and staff may not be familiar the features offered by the various devices available (Deng, Meng et al. 2002).

Similar trends are evident between both devices when assessing the number of failures and average severities per common phases of operation. For both devices, the most failures occurred during the main function of the endoscopic stapler, which involved cutting and firing on tissue (ETS $-56.8 \%$, Endo GIA $-56.6 \%)$. The second most common phase of operation in which failure occurred was the transition phase, which involved the reload, articulation, application, and removal of the device (ETS $31.5 \%$, Endo GIA - 29.2\%). The least common phase of operation in which failure occurred was the results and post-op phase, which involved the staple line (ETS - 11.7\%, Endo GIA - 14.2\%). Though, the results and post-op phase resulted in the highest average outcome severity (ETS - 3.46, Endo GIA - 3.50) for both devices compared to the other two phases. The transition phase resulted in the lowest average outcome severity (ETS - 2.83, Endo GIA - 2.85).

The frequencies and severities of adverse event outcomes associated with both endoscopic staplers were combined and assessed. Across all phases, a severe outcome (severity of 3) was the most common accounting for $60.3 \%$ of the outcomes assessed 
(transition $-17.4 \%$, main function $-35.3 \%$, results/post-op $-7.6 \%$ ). Of all failures reported, $47.8 \%$ that occurred during the main function resulted in a severity of 3 or higher. Of the failures reported, the proportion of the failures that resulted in a severity of 3 or higher for the transition phase and results and post-op phase were $21.0 \%$ and $12.9 \%$, respectively. All failures associated with the result and post-op phase were of severity 3 or higher. Across all phases, only one failure resulted in a mild outcome. Of the failures that occurred during the main function, $84.3 \%$ resulted in an outcome severity of 3 or higher. Of the failures that occurred during the transition phase, $69.1 \%$ resulted in an outcome severity of 3 or higher. Therefore when assessing each phase of operation individually, the failures associated with the results and post-op phase resulted in the most severe outcomes. Relative to the other phases, the main function phase resulted in the most dispersed severity outcomes on the 1-5 CTCAE v4.0 grading scale.

The outcome severity of endoscopic stapler failures was significantly different per common phase of operation in which the failure occurred $(p<0.05)$, deeming the association between the phase of operation in which an adverse event occurred and the severity of the outcome noteworthy. Due to the various factors involved in surgical stapling, such as staple height, tissue properties, accessibility, port placement, and so on, it can be difficult to narrow in on the root cause of an adverse event. Therefore, providing information regarding the severities associated with phases of operation and the failure modes within each phase can supply users and designers of the devices with insight regarding the most critical and risky aspects of the surgery. It is important for users to be aware of the types of severe outcomes that can result from device and user induced failures. 
Educating users about the potential failures and outcome scenarios is one way to bring awareness to the issues and further encourage the implementation of the preventative techniques as suggested in the scientific literature (Baker, Foote et al. 2004, Fuller, Paull et al. 2014, Mansour, El-Nashar et al. 2014). Users are encouraged to explore the available options for prevention, as many are specific to tissue types and procedures. Also, each technique can bring about its own additional risks that must be carefully considered and weighed by the user. For example, buttressing techniques are meant to make the staple line more uniform and provide additional support by placing thin sheets made of different material on one or both sides of the tissue to be stapled (Mery, Shafi et al. 2008). Buttressing has been associated with less bleeding from staples lines (Shikora, Kim et al. 2003, Angrisani, Lorenzo et al. 2004); however, the long term effects on healing, among other factors, are unclear (Mery, Shafi et al. 2008). Though relatively low compared to overall usage, endoscopic stapling device failures will occur. Therefore, it is even more important for users to be educated and trained in the most appropriate methods to implement in response to the issues that can arise in the surgical setting that are specific to endoscopic staplers.

\subsubsection{Energy-Based Tissue Sealer Adverse Event Outcome Severity}

The most severe failure mode outcomes associated with the Olympus Thunderbeat were related to an unspecific error that resulted in bleeding (average outcome severity of 5.00), seal failure with bleeding/leakage (3.53), and direct application (3.29). The outcome associated with the most severe failure mode involved death of the patient, yet the only details provided in the report indicated that bleeding 
resulted from an error during the activation phase. Further, recall from Section 2.3.3 that this failure mode only accounted for $1.2 \%$ of the total failures associated with the Thunderbeat. The most common failure mode associated with this tissue sealer was residual heat, which accounted for $28.4 \%$ of its total failures and resulted in an average outcome severity of 2.39. Seal failure with bleeding/leakage was the second most common failure mode associated with the Thunderbeat and resulted in one of the highest average outcome severities. Direct application, which results in thermal injury to adjacent tissue due to the unintended contact with the active probe during activation, resulted in one of the most severe outcomes and accounted for $8.6 \%$ of the Thunderbeat failures.

Similar to the Thunderbeat, the most severe outcomes associated with the Ethicon Harmonic Ace were the result of an unspecific error that resulted in bleeding (3.75), seal failure with bleeding/leakage (3.55), and direct application (3.33). The unspecific errors associated with bleeding accounted for 3.9\% of the Harmonic Ace failures, and one outcome involved death of the patient. The most common failure mode associated with this device was seal failure with bleeding/leakage, which accounted for over a quarter of its failures (28.4\%) and also resulted in one of the highest average outcome severities. Direct application accounted for $2.9 \%$ of Harmonic Ace failures. Residual heat and the failure to seal or seal properly were also common failure modes of this tissue sealer with each accounting for $15.7 \%$ of the failures and resulting in average outcome severities of 2.44 and 2.81 , respectively.

Seal failure with bleeding or leakage was a common failure mode that resulted in severe outcomes for both the Thunderbeat and Harmonic Ace devices. Adverse event 
outcomes associated with tissue sealers that utilize either ultrasonic or bipolar energy or both commonly involve bleeding as a result of failure (Overbey, Townsend et al. 2015). While concerning, it is important to emphasize the specific function of these devices, which involves sealing and transecting vessels. Failures can be prone to association with bleeding and leakage outcomes due to the very nature of the surgical tasks these devices are relied upon to carry out. Though, the patient risks are heightened for failures that occur post-operatively. Thorough inspection of the seal prior to the completion of a procedure is necessary in order to prevent post-operative seal failure that may require reoperation. Users must be aware of the sealing capabilities of their device(s) of choice given that various brands and types of tissue sealers specify the range of vessel diameters in which they are intended to seal. Further, users must have the experience and judgement practices that allow proper assessment regarding the use of tissue sealers for various applications, tissue types, and tissue/vessel sizes.

Residual heat was a common failure mode associated with the tissue sealers and resulted in an average severity outcome between moderate (severity grade of 2) and severe (severity grade of 3) on the CTCAE v4.0 grading scale. Across both devices, only one outcome resulted in death and no life-threatening outcomes occurred. Specifically, the death was reportedly due to a post-operative leak that originated from a burn on the small bowel. Burns to hollow structures, such as the bowel, are deemed to be of the highest risk given the potential for post-operative leakage and infection. Burns due to residual heat were reported to have affected the patient's internal and external organs in addition to the surgical team's skin. Each of the consequences can result in a range of outcome severities depending on the location and degree of the burn due to residual heat 
of the device's distal tip. In a 2011 study, Govekar et al. determined that even 20 seconds after activation the tip of ultrasonic energy devices heated tissue more than $20^{\circ} \mathrm{C}$ from baseline temperature, which further emphasizes the need for cautious handling of devices that utilize ultrasonic technology after activation (Govekar, Robinson et al. 2011).

Assessing both the frequency of failures modes and their corresponding outcome severity is necessary to provide an inclusive perspective on adverse event outcomes. Various failure modes may be uncommon, yet result in severe outcomes when they do occur. Failures involving direct application and an unspecific error that resulted in bleeding during activation were not the most common failures modes associated with the tissue sealing devices, yet their outcomes were among the most severe. Unfortunately, various event descriptions were not specific enough to identify the error that resulted in bleeding; however, it is evident that bleeding during the activation phase is concerning due to the life-threatening and morbid outcomes that can result. The outcome severities herein provide additional noteworthy information in the assessment of adverse event reports that frequencies and proportions alone cannot deliver. A comprehensive evaluation of the adverse events associated with surgical devices should at least include the types of failures modes and their relative frequencies, the range of outcome severity associated with the failure modes, and average outcomes severities per failure mode and phase of operation.

The majority of failure occurred during the main function for both the Thunderbeat and Harmonic Ace devices, which involved the activation phase (Thunderbeat $-43.4 \%$, Harmonic Ace $-46.1 \%$ ). The second most common phase of failure associated with the Thunderbeat was the transition phase (36.1\%), and the least 
common was the results and post-op phase (20.5\%). Conversely, the second most common phase of failure associated with the Harmonic Ace was the results-post-op phase (30.4\%), and the least common was the transition phase $(23.5 \%)$. On average, the most severe outcomes occurred during the results and post-op phase (Thunderbeat -3.70 , Harmonic Ace - 3.52). The transition and main function phases associated with the Thunderbeat resulted in similar average outcome severities of 2.37 and 2.36, respectively. The main function resulted in more severe outcomes on average than the transition phase for the Harmonic Ace at 2.40 and 2.08, respectively. For both devices, the transition and activation phase average outcome severities fell between moderate (severity of 2) and severe (severity of 3) on the CTCAEv4.0 grading scale, while the results and post-op phase average outcome severities fell between severe (severity of 3 ) and life-threatening (severity of 4). Adverse event outcomes associated with the final seal (results) and those that occur post-operatively are more severe on average than those that occur during the transition and main function phases of tissue sealer operation.

The frequencies and severities of adverse event outcomes associated with both tissue sealers were combined and assessed. Across all phases, a severe outcome (severity of 3) was the most common accounting for $45.4 \%$ of the outcomes assessed (transition $11.9 \%$, main function $-20.0 \%$, results/post-op $-13.5 \%$ ). The results and post-op phase for tissue sealers resulted in the highest overall average severity of 3.58 and accounted for the most outcomes graded as severity 3 or higher relative to the total number of failure outcomes assessed (25.4\%). Of the failures that occurred during the transition phase, $42.6 \%$ resulted in an outcome severity of 3 or higher. $54.2 \%$ of the failures that occurred during the main function resulted in an outcome severity of 3 or higher. Of the 
failures that occurred during the results and post-op phase, $97.9 \%$ resulted in an outcome severity of 3 or higher.

The outcome severity of tissue sealer failures was significantly different per common phase of operation in which the failure occurred $(p<0.05)$. Therefore, the phases of tissue sealer operation were associated with the severity of the outcomes that occur in each phase. It is important to consider this association with both the frequency and types of failure that occur during each phase of operation. Users must be knowledgeable of the risks associated with each phase of operation in addition to the most common types of failure that occur during each phase in order to heighten awareness throughout various stages of a procedure and device use.

\subsubsection{Surgical Device Adverse Event Outcome Severity}

The failure mode outcome severity data for endoscopic staplers and tissue sealers was combined and analyzed per common phase of operation. The majority of failures occurred during execution of the devices' main function $(51.3 \%)$. The main function of both surgical devices involves securing (either via stapling or sealing) and transecting tissue (either via knife or thermal/mechanical energy). The average severity of the outcomes that occurred during the devices' main function was between moderate and severe at 2.80. Relative to the total number of failures assessed, $37.2 \%$ of the failures during the main function resulted in a severity of 3 or higher. Across both devices, $72.4 \%$ of the number of failures that occurred during the main function resulted in a severity of 3 or higher. 
The transition phase was the second most common phase of operation in which failure occurred, accounting for $29.8 \%$ of failures. Transition failures vary per device type, but in general this phase refers to any handling of the device during the procedure excluding execution of the main function. The average outcome severity of the failures that occurred during transition was the lowest relative to the other phases of operation (2.57). Of the total number of failures, the transition phase accounted for the lowest number of outcomes that resulted in a severity of 3 or higher (17.1\%). Similarly, the transition phase accounted for the least amount of outcomes that resulted in severity of 3 or higher relative to the number of outcomes that resulted within that phase $(57.4 \%)$. The least amount of life-threatening outcomes and deaths occurred during transition relative to the other phases of operation, which is likely due to the counteractive techniques available for the user to employ during these circumstances. Relative to main function and post-op failures, users are typically able to resort to less severe tactics during the reaction to many types of transition failures.

The least amount of failures occurred during the results and post-op phase $(18.8 \%)$; though, the failures that occurred during this phase resulted in the highest average outcome severity of 3.55. This phase included failures that occur after the main function was carried out (i.e. seal or staple line after activation and firing, respectively) and adverse event outcomes that occurred post-operatively. Adverse events associated with the results of device use post-operatively mainly involved surgical ligation failure resulting in leakage or bleeding. Of the total failures assessed, $18.6 \%$ of the results and post-op phase failures resulted in a severity grade of 3 or higher. Further, of the failures that occurred during the results and post-op phase, $98.7 \%$ resulted in an outcome severity 
of 3 or higher. Seal or staple line failure after the completion of a procedure involves deployment of undesirable tactics, such as reoperation, and subjects the patient to high risk related to leakage and blood loss.

The percentage of outcomes that resulted in a severity of 3 or higher were determined for endoscopic staplers, tissue sealers, and for both device types. $81.7 \%$ of endoscopic stapler failures resulted in an outcome severity of 3 or higher, and $62.2 \%$ of tissue sealer failures resulted in an outcome severity of 3 or higher. Across both device types, $72.8 \%$ of failures resulted in an outcome severity of 3 or higher. Determining the number of failures that result in an outcome severity of 3 or higher was of interest considering these were failures that resulted in a medically significant outcome deemed either severe, life-threatening, or morbid. A severe outcome indicated that either invasive intervention was required or the procedure was altered in some way due to the failure, whether requiring a conversion from laparoscopic to open surgery or a procedural delay. These outcomes in addition to those even more severe (severity grade of 4 and 5) are the most concerning due to their nature and the associated risks. Therefore, it is essential to assess these occurrences per phase of operation and device type.

The outcome severity of failures associated with both device types was significantly different per phase of operation in which the failure occurred $(p<0.05)$. Therefore, the phases of surgical device operation were associated with the severity of the outcomes that occurred in each phase based on the endoscopic stapler and tissue sealer data. While many studies have assessed the types of failures associated with these devices, and in some cases the frequency, no study to date has incorporated a severity assessment of the outcomes associated with the failures and phases of device operation 
(Tucker and Voyles 1995, Brown and Woo 2004, Fuller, Paull et al. 2014, Mansour, ElNashar et al. 2014, Overbey, Townsend et al. 2015, Riggs, Bohm et al. 2016). Not only do designers and medical professionals need to be aware of the type and frequency of failures, but they also must be knowledgeable regarding the level of risk posed by each failure.

Whether inherent to device design or the nature of the specific patient's tissue, unanticipated and unexpected outcomes associated with device use will occur. Despite the low failure rate relative to overall usage, users must not only be knowledgeable in proper functioning and use of the devices, but also in the potential adverse event outcomes related to the most common types of failure. The more tools and methods a surgeon can deploy in reaction to the various potential device failures, then the less severe the outcome will likely be. A surgeon's awareness of proper device use and function in addition to alternative procedure methods and bleeding management techniques contribute to prevention of adverse events, especially those that may have resulted in an event of severity grade 3 or higher.

Steps should be taken to not only reduce the severity, but also work towards complete prevention of an adverse event. The method described is the first step towards utilizing severity grades in the assessment of adverse event outcomes retrieved from the FDA MAUDE associated with minimally invasive surgical devices. This work initiated a new approach to enhance risk awareness and prevention techniques through assessment of the severity of the various failure mode outcomes derived from adverse event reports by applying the CTCAE grading method. The failure mode type, frequency, and outcome severity data presented efficiently outlines and provides inclusive content to aid 
designer and medical professional's education and awareness of the risks associated with various device types and procedures.

Knowledge and experience is crucial for the recognition of the onset of specific failures, which reiterates the importance of filling the knowledge gap related to device use, performance, tissue interaction, potential failure, and preventative action (Deng, Meng et al. 2002, McColl, Karmali et al. 2009, Feldman, Fuchshuber et al. 2012, Feldman, Brunt et al. 2013, Madani, Watanabe et al. 2014, Riggs, Bohm et al. 2016). The application of an outcome severity grading method serves as a contribution to filling the knowledge gap regarding device use and failure in addition to bridging the gap between manufacturers and users. A collaborative effort is required among various disciplines in order to make significant headway in preventing device and user induced errors, especially as they relate to the devices used in minimally invasive surgeries. It is important for users to be aware of the types of failures that can occur with device usage and the corresponding general severity that can potentially result. The information presented can contribute to the enhancement of a user's approach when encountering the various phases of operation outlined herein. User awareness should be heightened during all phases of operation, but especially in situations where the most severe types of failure modes are prone to occur.

The application of severity to phases and failure modes can be applied to devices outside of the four in this study. It is likely that the severities identified apply to the various brands of devices available within the two classes, and the insights provided by this work can be applicable even as the technology advances. Manufacturers can utilize severity grading data as it relates to adverse events in order to determine the device 
features that can be implemented to reduce or prevent specific failure modes that may tend to be the most severe. It can also provide insight into which features may or may not be attributing to issues. For instance, some staplers have a lockout mechanism that is intended to prevent an empty reload (staples deployed on a previous fire) from being fired. However, it has been demonstrated that users are capable of overriding this lockout mechanism by applying excessive force and consequentially cutting tissue without applying staples (Deng, Meng et al. 2002). Significant force to fire should be an indication of improper firing conditions, yet some users may proceed in their attempt to fire. Therefore, not only is a user knowledge gap exhibited, but also a gap between the manufacturer and user regarding device performance, function, application, and instructions for use.

The manufacturers have a responsibility to recognize improper use and the level of success exhibited by device safety features in order to address the current issues and implement countermeasure improvements in future designs. Various tools are in place to assist the communication between the user and manufacturer regarding device performance, yet further developments are necessary based on the findings related to both user and device based failure modes and their corresponding severities. The knowledge gaps exhibited also demonstrates the need for a comprehensive understanding of not only the specific types of failure modes prone to certain surgical devices, but also the associated outcome severities that can potentially result in the operation setting.

\subsection{Outcome Severity Study Limitations and Future Work}


The limitations of this work are consistent with those of the failure mode study listed in Section 2.5. Event descriptions within the reports were not all sufficient for determining the exact outcome. Insight gained from the manufacturer narrative was used in some of these incidences to identify the nature of the adverse event outcome. In some cases when the consequence was not explicitly stated, the severity grade was inferred based on the context provided in the event descriptions and manufacturer narrative. All outcomes could not be directly linked to the user or device based failure. In some cases, the terminology and descriptions provided were not easily interpretable, and best judgement in the severity of the outcome was used given the information provided as it relates to the CTCAE grading criteria. Therefore, it is possible that misclassification of failure mode outcome severity could have occurred leading to an improper overall assessment of outcome severity. Terminology associated with specific failure modes and adverse event outcomes (including patient consequence) needs to be more consistent, and the current terminology suggestioned in literature should be utilized by user and facility administration when submitting reports to the FDA MAUDE (Brown and Woo 2004, Fuller, Paull et al. 2014, Overbey, Townsend et al. 2015, Riggs, Bohm et al. 2016).

The severity grades applied to the adverse event outcomes were carried out by one investigator. This is a limitation of this work as best practice involves multiple investigators who demonstrate a satisfactory inter-rater reliability. Future work should involve multiple investigators in the application and consensus of severity grades.

It has been demonstrated that applying severity grading methods to the adverse event reports in the FDA MAUDE is useful for users and manufacturers as one way to help bridge the knowledge gap. Though, resource and budget limitations did not allow 
for the assessment of additional devices that were necessary for other components of the overall research goals. Not only should other brands of tissue sealers and endoscopic staplers be investigated, but also additional device classes. The application of the CTCAE to the FDA MAUDE database reports should be expanded in order to bring awareness regarding the severity associated with medical device failure modes in various procedural settings.

The CTCAE grading was the most appropriate grading method for the intraoperative surgical complications associated with medical device adverse events. However, the all of the criteria outlined for this method was not directly applicable in all cases (i.e. criteria referencing how the adverse outcome affects activities of daily living). Proper grading classification was inferred based on the general criteria outlined for each severity grade in the CTCAE v4.0. This work assessed the severity of each adverse event as it relates to failure mode consequence. While this work alludes to the severity that would likely be associated with specific failure modes, the predictability of severity grades per various failure modes should be analyzed more in depth with applicable statistical analyses. Such analyses would likely require a larger sample size. Further investigation could also be valuable that assesses the severity of outcomes due to failures and how it relates to particular applications, tissue types, and procedures. 


\section{CHAPTER IV \\ DEVICE DESIGN COMPLEXITY SCORE}

\section{$\underline{4.1 \text { Complexity Study Overview }}$}

The use of medical devices designed specifically for MIS is increasing along with their application to more wide-ranging procedures due to rapid technological and design evolution (Deng, Meng et al. 2002, Janetschek, Bagheri et al. 2003, Swanstrom, Kozarek et al. 2005, Feldman, Fuchshuber et al. 2012, Feldman, Brunt et al. 2013, Lyons and Law 2013, Chekan and Whelan 2014). Design and development of new and modified surgical devices is vital to the enhancement of procedural outcomes, patient safety and wellbeing, and surgeons' capabilities in the operation room. Investigation into a method to accurately assess product complexity in particular will enable optimal design and manufacture of various classes of medical devices through design variable modifications. Such investigation can also provide a better understanding of the design aspects that can affect product performance. Therefore, identifying the complexity of minimally invasive surgical devices is crucial in the early design stages in order to ensure the effectiveness and safety of a device's final design. The design variables associated with surgical devices are of heightened interest considering they are increasingly relied upon to function properly during the execution of intricate minimally invasive procedures 
(Deng, Meng et al. 2002, Janetschek, Bagheri et al. 2003, Johnson, Couture et al. 2007, Feldman, Fuchshuber et al. 2012, Kwazneski, Six et al. 2012).

Specific Aim 3 is addressed in this chapter, and the purpose of this aim was to characterize the design complexity of endoscopic staplers and energy-based tissue sealers used in MIS using device component decomposition and functional modeling. Two endoscopic staplers and two energy-based tissue sealers and were reverse engineered and a Design Structure Matrix (DSM) was constructed for each. The DSMs were used in order to generate a complexity score for each device using the connectivity algorithm developed by Summers and Ameri (Summers and Ameri 2008). The connectivity algorithm was also applied to generate a complexity score for the functional model that represents each device type. The functional aspects, design features, and complexity scores of each device were compared and discussed.

The nature of various aspects of device use potentially affect procedural outcomes, such as the instructions for use, the procedural tasks and requirements, the clinical environment, and the device design. A multi-domain approach involving the intersection of medical and engineering design knowledge is necessary in order to quantify the complexity of each of these aspects and begin addressing how they relate to the outcomes of surgical device use and performance. This study is the first to apply an existing method of quantifying design complexity to minimally invasive surgical devices and investigate the feasibility of the application in this domain.

\subsection{Complexity Scoring Methods}




\subsubsection{MIS Devices Obtained for the Complexity Analysis}

\subsubsection{Endoscopic Staplers}

Two endoscopic staplers were obtained for the complexity analysis: the Covidien Endo GIA ${ }^{\mathrm{TM}}$ Universal Stapler with the Covidien Endo GIA ${ }^{\mathrm{TM}}$ Universal Roticulator $^{\mathrm{TM}}$ Reload (referred to as Covidien Endo GIA in sections hereafter) and the Ethicon EndoSurgery ENDOPATH® ETS Endoscopic Articulating Linear Cutter (hereafter referred to as Ethicon ETS). The Ethicon ETS expired in 2014 and was purchased in the original packaging and box on eBay for $\$ 69.99$ plus $\$ 12.99$ shipping. The Covidien Universal handle had an expiration date of 02/2017 and was purchased in the original packaging on eBay for $\$ 50.00$ plus $\$ 11.00$ shipping. The Covidien Reload expired in 2014 and was purchased in the original packaging on eBay for $\$ 29.99$ plus $\$ 10.00$ shipping. The Covidien and Ethicon stapling devices were purchased from eBay in February 2016 and are shown removed from the original packaging in Figures 9 and 10, respectively.

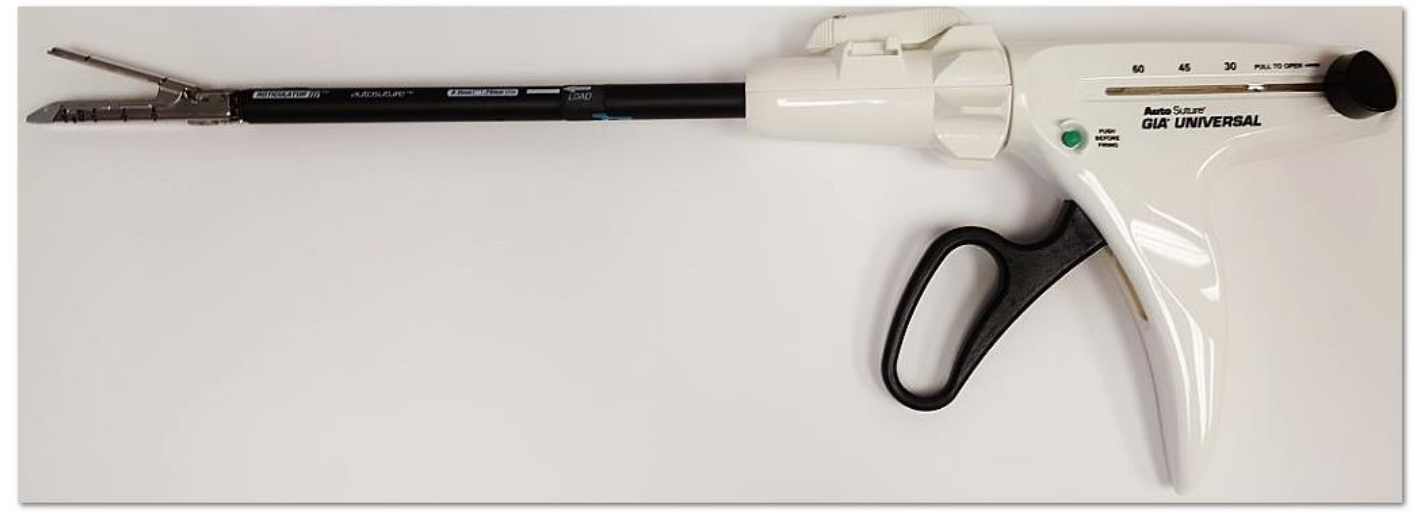

Figure 9. The Covidien Endo GIA ${ }^{\mathrm{TM}}$ Universal stapler and the Covidien Endo GIA ${ }^{\mathrm{TM}}$ Universal Roticulator ${ }^{\mathrm{TM}}$ reload attachment. 


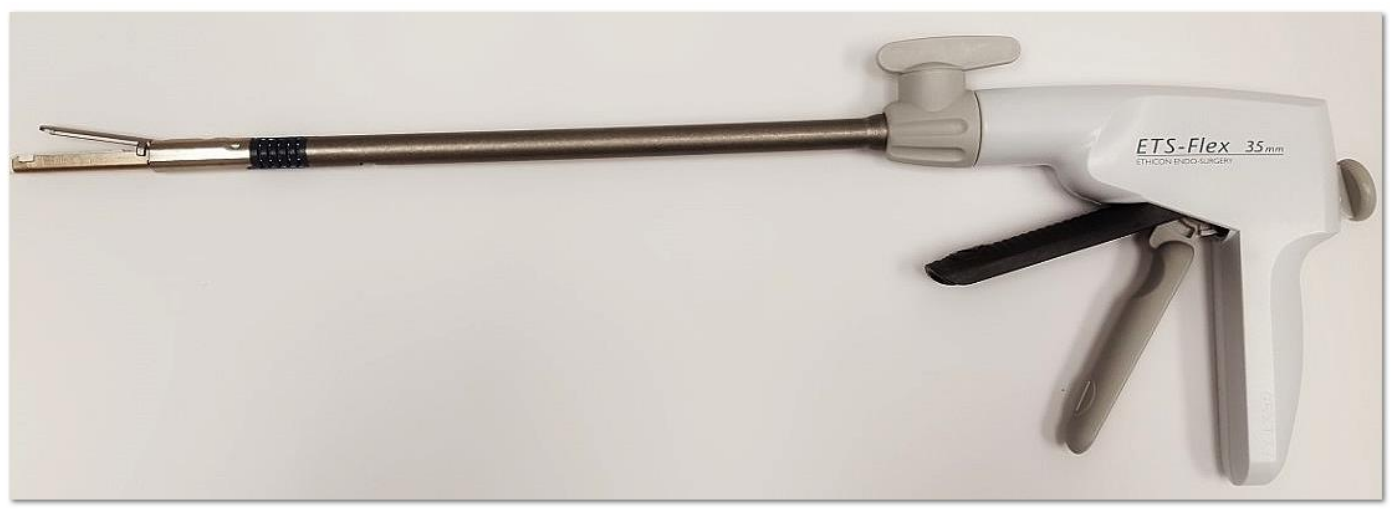

Figure 10. Ethicon Endo-Surgery Endopath® ETS Endoscopic Articulating Linear Cutter.

Each device was fired on a double layer of tissue paper prior to deconstruction and the user experience was noted during each step of use. The devices were disassembled in order to develop a list of components and determine their interconnectivity.

\subsubsection{Energy-Based Tissue Sealers}

The following energy-based tissue sealers were purchased for the analysis: Olympus Thunderbeat Surgical Tissue Management System (referred to hereafter as Olympus Thunderbeat) and the Ethicon Endo-Surgery Harmonic Ace Tissue Sealing Device (referred to hereafter as Ethicon Harmonic Ace). The Olympus Thunderbeat shown in Figure 11 had an expiration year of 2017 and was purchased on eBay in March 2016 for \$175. The Ethicon Harmonic Ace shown in Figure 12 expired in 2013 and was purchased on eBay in March 2016 for $\$ 180$ plus $\$ 5.99$ shipping. 


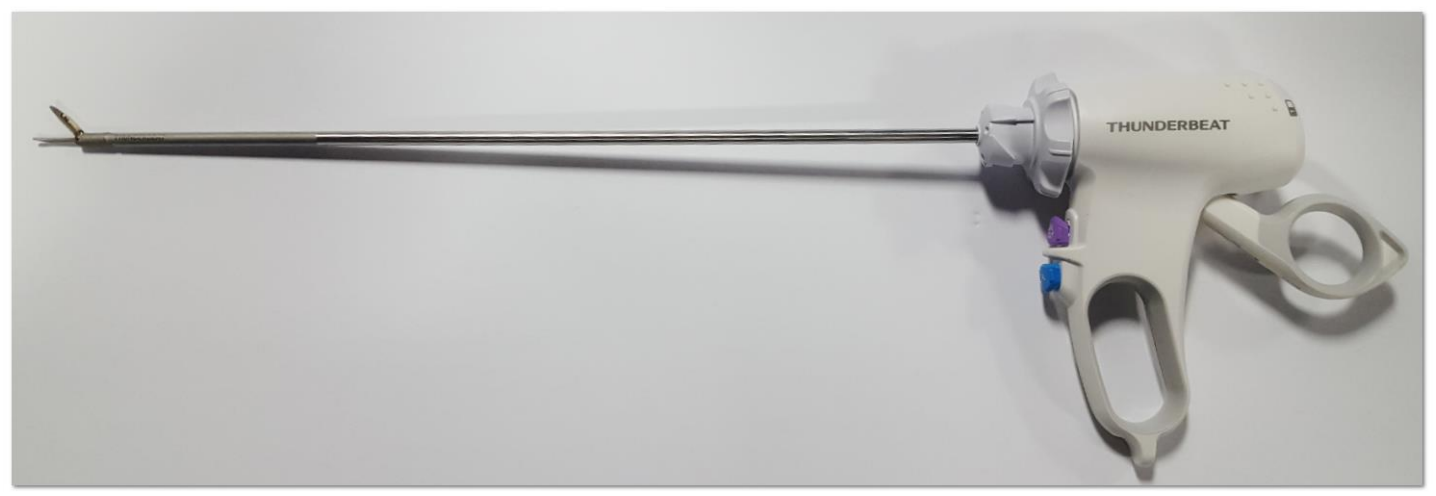

Figure 11. Olympus Thunderbeat Surgical Tissue Management System.

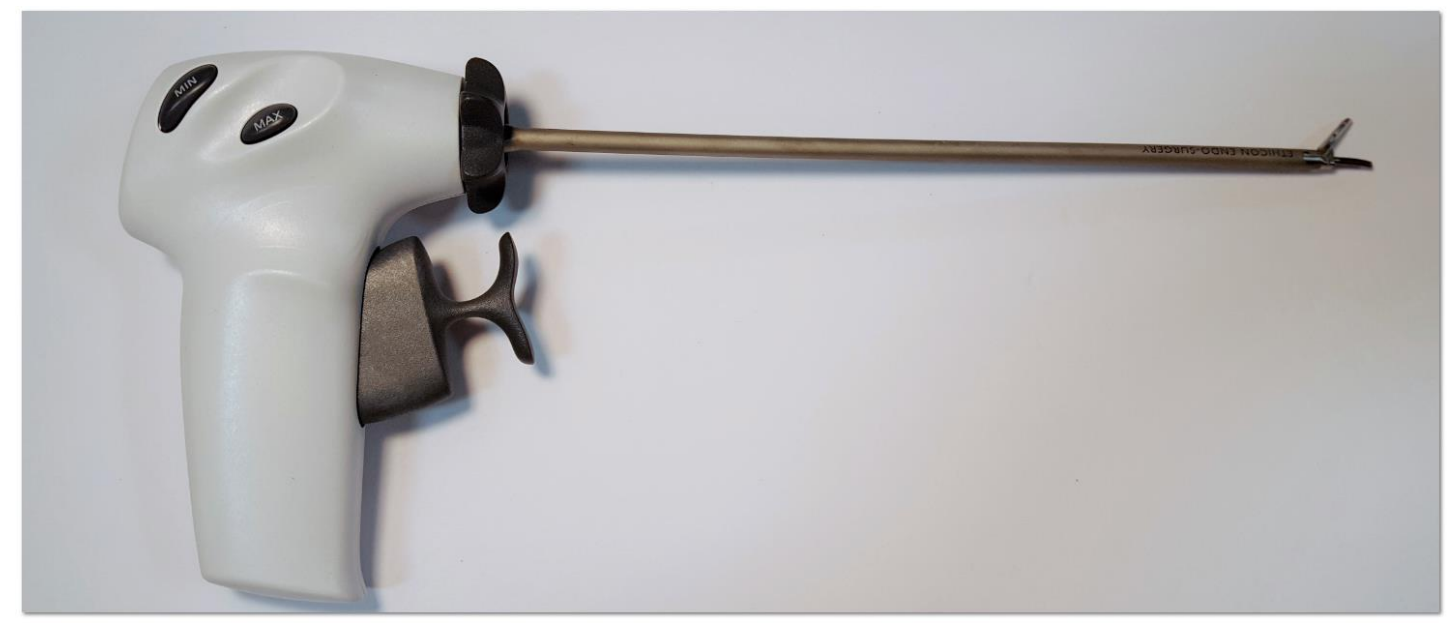

Figure 12. Ethicon Harmonic Ace tissue sealer.

The devices were reverse engineered in order to develop a list of components and determine their interconnectivity. Component decomposability was obtained in order to construct a DSM for each device.

\subsubsection{Connectivity Algorithm}

Device patents were used to assist proper reassembly and identification of components (Hmtema 1998, Huitema, Nalagatla et al. 1998, IV, Setser et al. 2005, 
Marczyk 2006, Scirica 2010, Hall, Tanguay et al. 2011, Soltz and Broom 2012). A DSM was created for each device, and a complexity score was calculated using coupling complexity, which accounts for a measure derived from the connections between variables at multiple levels represented in a graph-based format (Carrascosa, Eppinger et al. 1998, Eppinger and Browning 2012). A connectivity algorithm was proposed in the literature to measure the connectivity of an entity-relationship graph via removal of relationships between entities until the graph is decomposed into individual entities (Summers and Ameri 2008). Graphs are repeatedly separated into sub-graphs until single nodes remain. Once the algorithm has been applied, a decomposability score results, which measures how difficult it is to take the product or system apart piece by piece. Complexity increases with the more steps, relationship sets, or relationships per separated element that are required to decompose the product or system entirely. The connectivity algorithm was outlined in Table 5 in Section 1.2.7.3.

The DSM for each device was converted into a bipartite graph in order to apply the connectivity algorithm. Additionally, a bipartite graph was derived from each device functional model. An example of a bipartite graph is shown in Figure 13. Components are represented on the left side of the graph, and relationships are depicted on the right. 


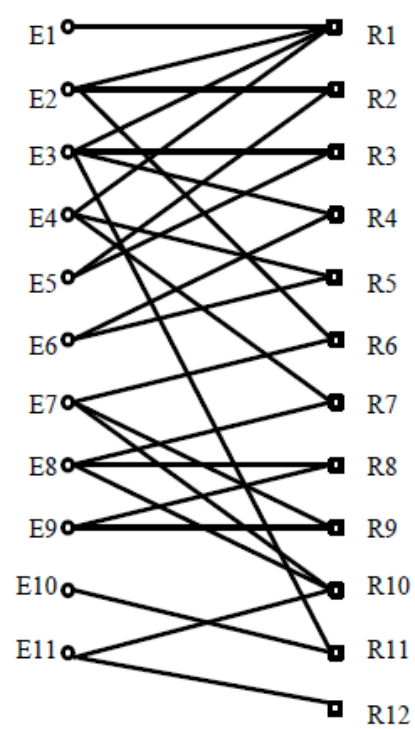

Figure 13. Bipartite graph (entity relationship graph) (Summers and Ameri 2008).

A Matlab program was coded by the developers of the connectivity algorithm at Clemson University given that manual calculation of complexity scores from bi-partite graphs can be cumbersome for systems and products that involve many components and connections. The developers permitted use of the program in this study in order to obtain complexity scores based upon connectivity. The program reads each device DSM from an excel file, converts it into a bipartite graph, and calculates the complexity score. Summers and Ameri provide step-by-step instructions and examples regarding application of the connectivity algorithm to the bipartite graph in order to obtain a complexity score as executed by the program (Summers and Ameri 2008). The functional aspect of a device contributes to overall design complexity. Therefore, the algorithm was also be applied manually to the functional models to determine their complexity. 


\section{$\underline{4.3 \text { Complexity Results }}$}

\subsubsection{Functionality, Reverse Engineering, and DSMs}

Endoscopic staplers and energy-based tissue sealers share a common functional requirement to secure and separate tissue. However, each device executes the function utilizing dissimilar mechanisms. The two classes of devices are applicable to different tissue types and procedures. The differing mechanisms between device types and device brands involved in executing the main function are discussed.

\subsubsection{Endoscopic Stapler Function and DSM}

The overall function and objective of each endoscopic stapler is identical, yet different manufacturers' staplers vary in terms of their components, attachments, features, and overall design. Ethicon Endo-Surgery generally supplies a full stapler that requires reloading of individual stapler cartridges into the elongate channel of the device jaw. After an Ethicon stapler is fired, it requires a new staple cartridge in order to be used again in the same procedure. The device can then be reloaded and fired in the same patient, meaning the same jaws, articulation components, and knife can be utilized in a single procedure. Covidien generally supplies a handle with a shaft portion that connects to a separate reload. The reload is packaged and sold separately. Replacing a Covidien reload involves new jaws, distal articulation components, and knife for the next fire during the same procedure. Loaded jaws of each device are shown in Figures 14 and 15, respectively. 


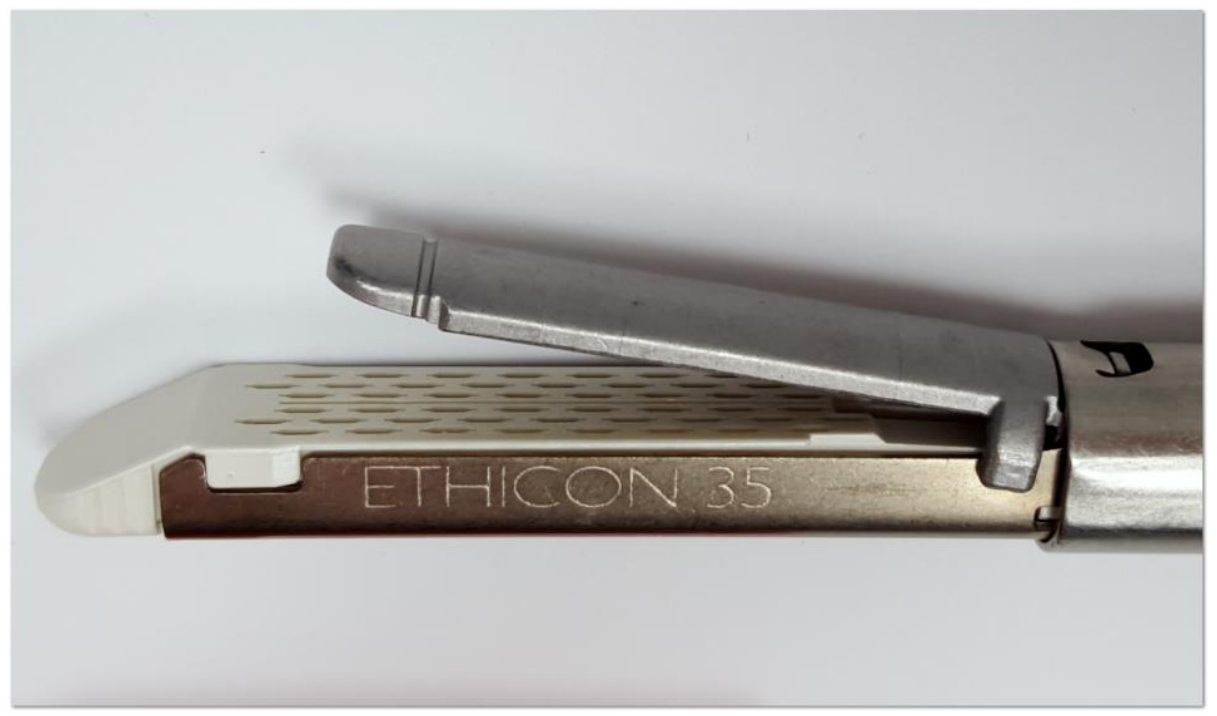

Figure 14. Ethicon ETS loaded jaw.

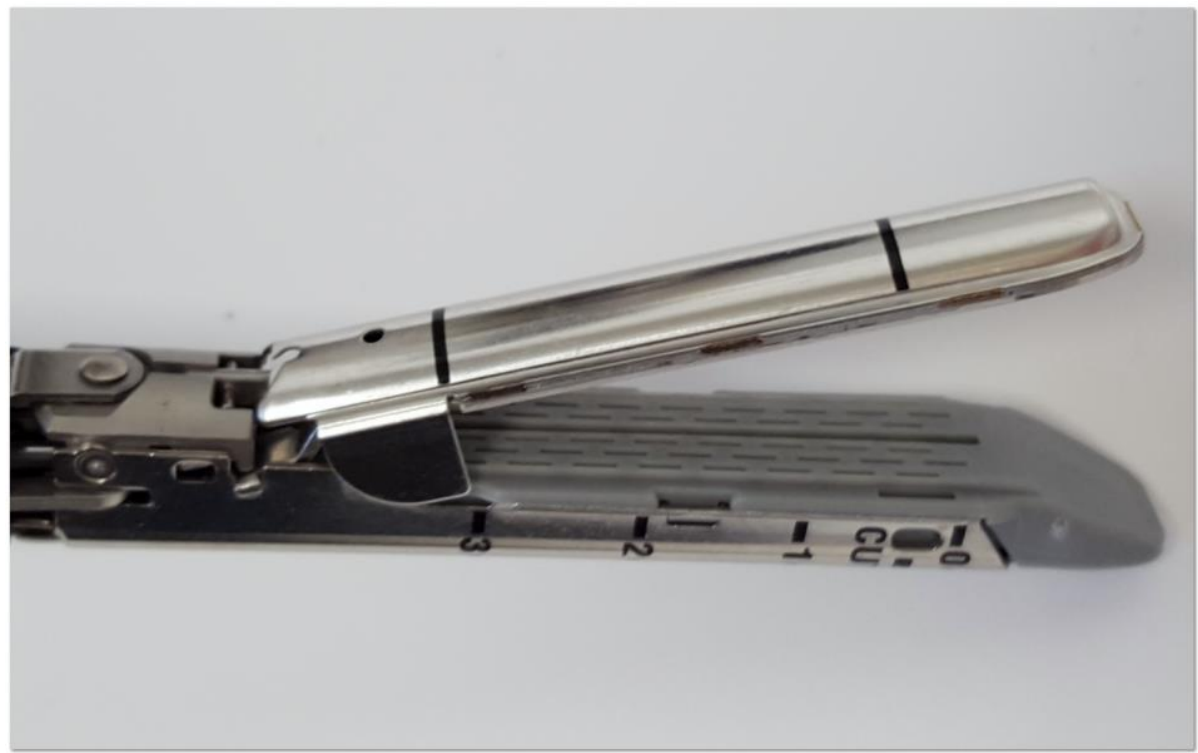

Figure 15. Distal Covidien Endo GIA reload.

The firing mechanism varies between Ethicon and Covidien devices (Covidien 2017, Ethicon 2017). To fire the Ethicon stapler, a clamping trigger is pulled and locked closed. After waiting for the tissue to compress, the firing trigger is squeezed until the 
firing sequence is complete. To release the clamping trigger (and jaws), a button on the back of the handle is pressed. The Covidien stapler has one trigger, which is used to clamp and fire. Once tissue is clamped, the user must press a button on the side of the device to allow stapling. The same handle is squeezed and released multiple times to advance the knife through the tissue. Once the firing sequence is complete, the return knobs are pulled back to retract the knife and open the jaws. The handles and triggers for the Ethicon ETS and the Covidien Endo GIA are shown in Figures 16 and 17, respectively.

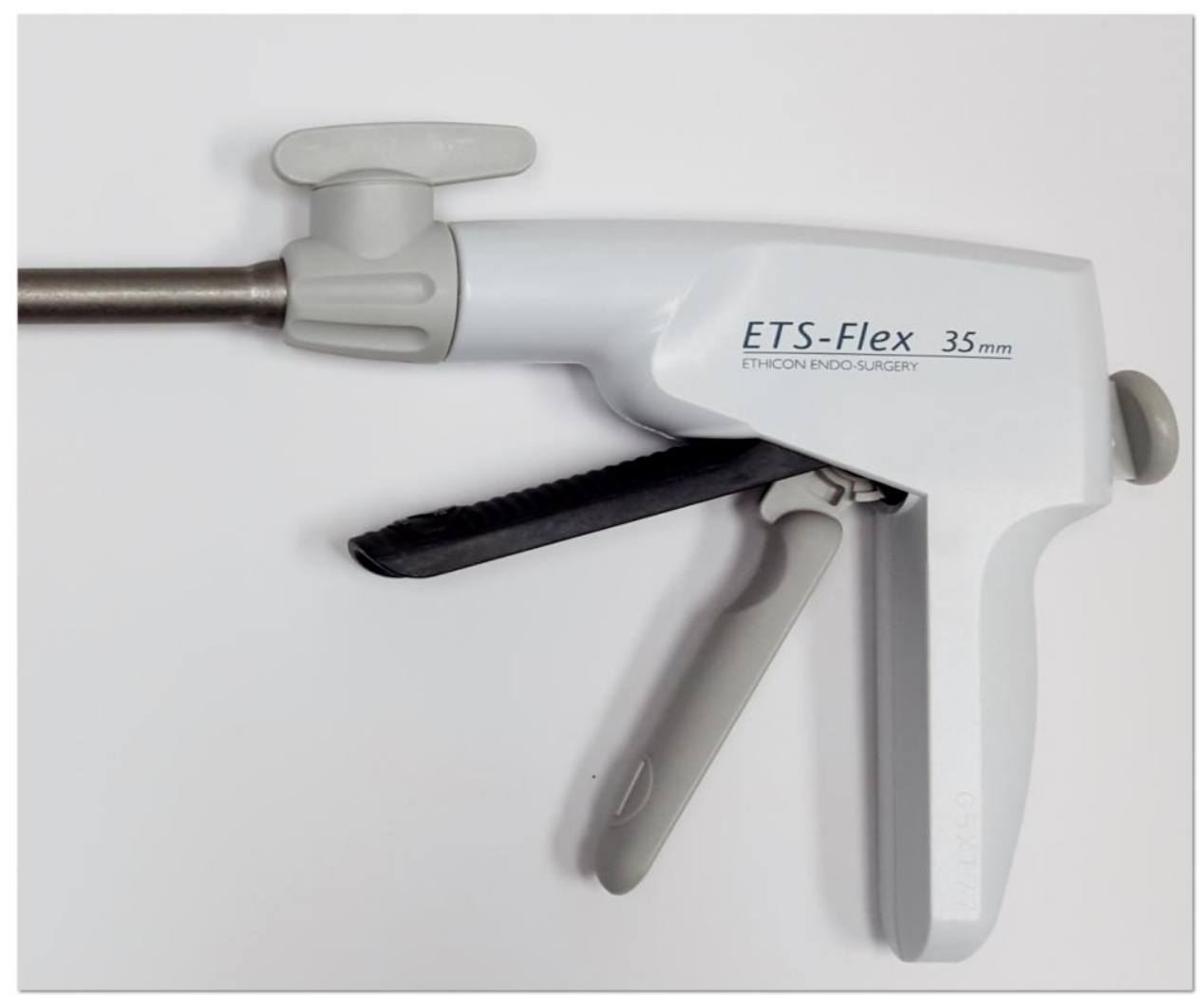

Figure 16. Ethicon ETS handle with triggers and release button. 


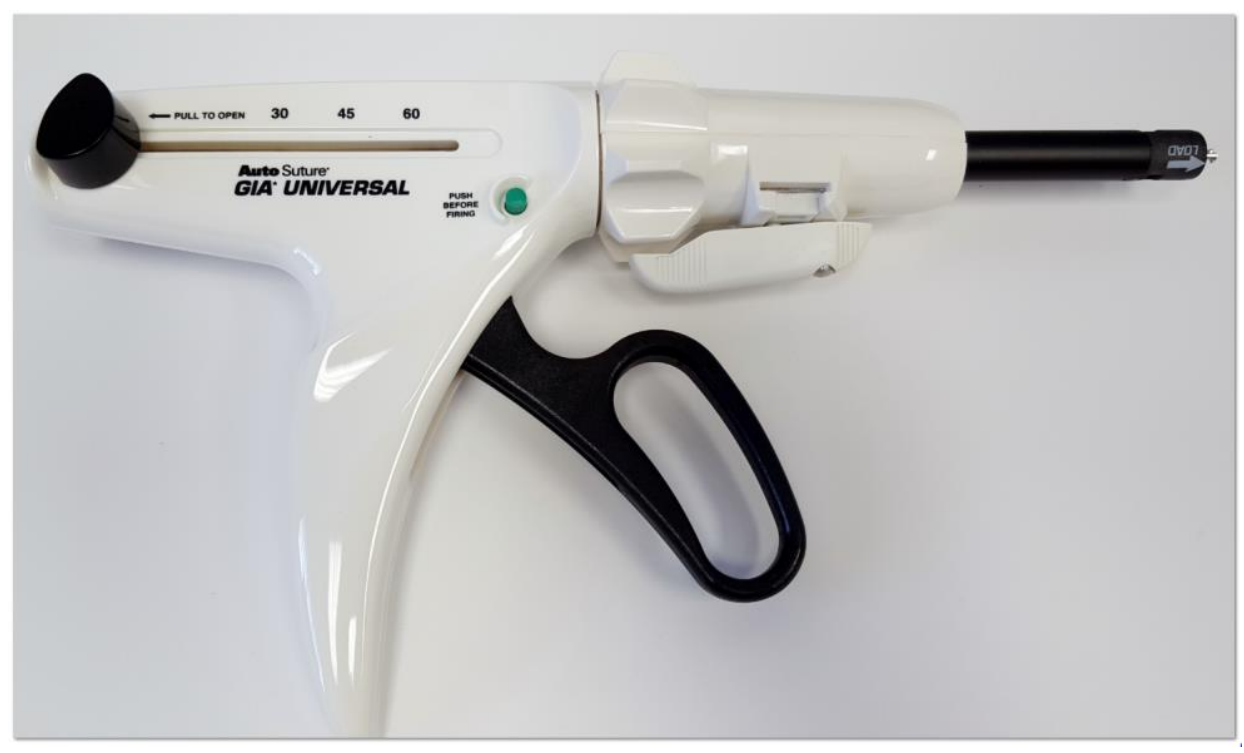

Figure 17. Covidien Endo GIA handle with trigger and return knobs.

The type of components and mechanisms for shaft rotation, articulation, and safety features vary per device brand as well. Each endoscopic stapler brand offers options for shaft lengths, staple line lengths, and staple heights to suit various applications and tissue types (Covidien 2017, Ethicon 2017). The functional model for endoscopic staplers is shown in Figure 18. 


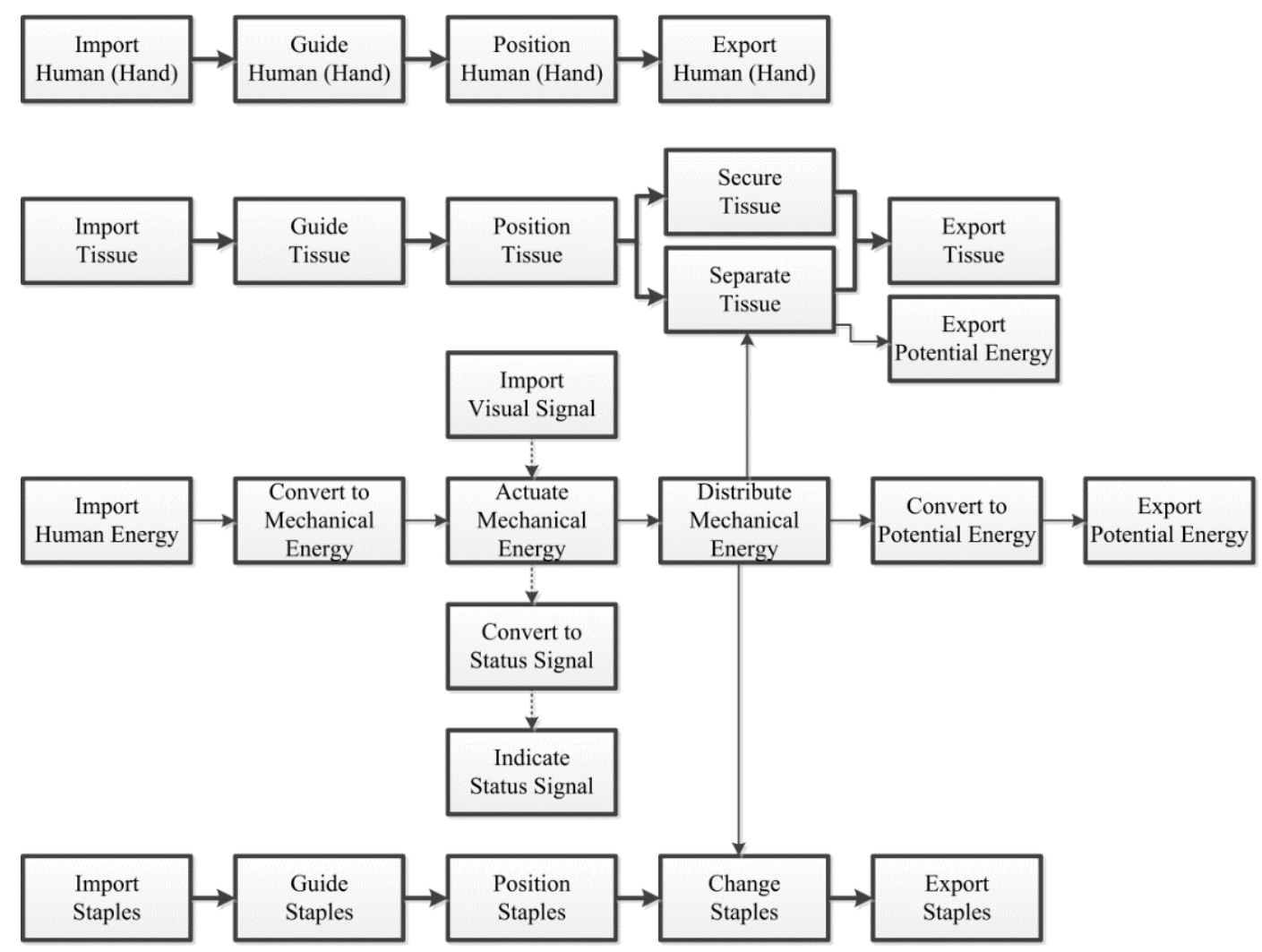

Figure 18. Endoscopic stapler functional model.

The endoscopic stapler functionality involves 25 functions and 23 connections between them. The DSM derived from the functional model is included as an electronic attachment. The devices were reverse engineered to assess connectivity. The Ethicon ETS had 38 total components identified for the DSM, and an exploded view of the device is shown in Figure 19. The Covidien Universal handle and reload were comprised of 47 components and 42 components, respectively, resulting in a total of 89 components. An exploded view of the Covidien components is shown in Figure 20. The staples were recognized as a single part in each device DSM, as well as the pieces that drive the staples upward towards the anvil identified as "drivers" or "pushers." 


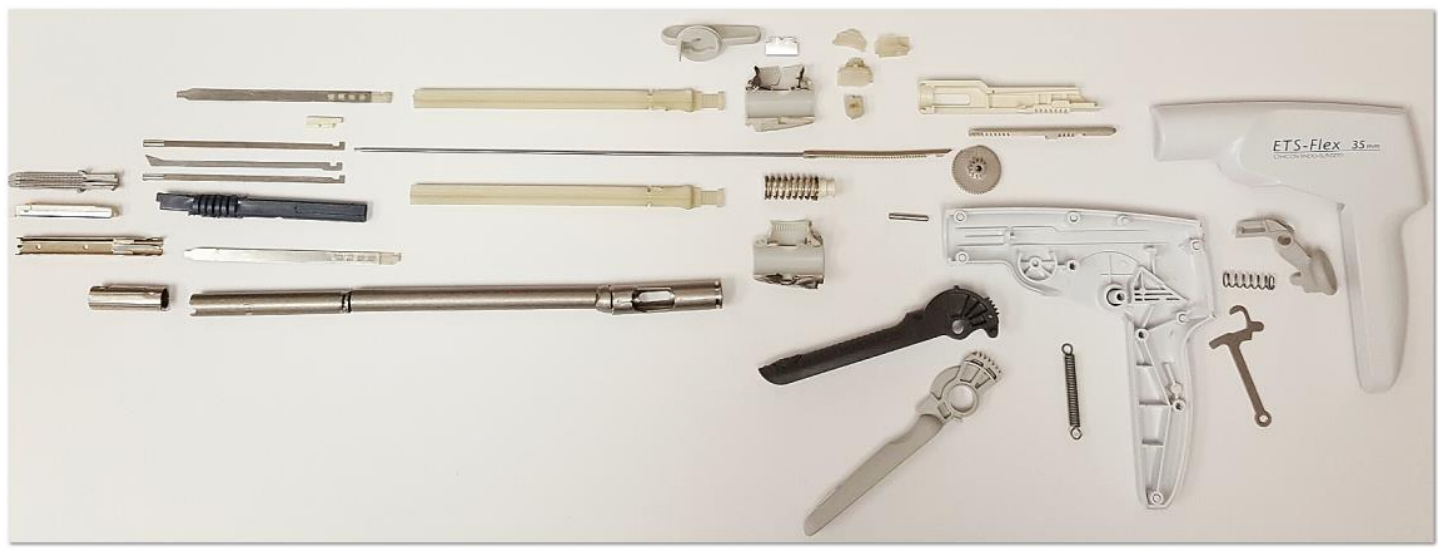

Figure 19. Ethicon Endo-Surgery Endopath® ETS Endoscopic Articulating Linear Cutter exploded view.

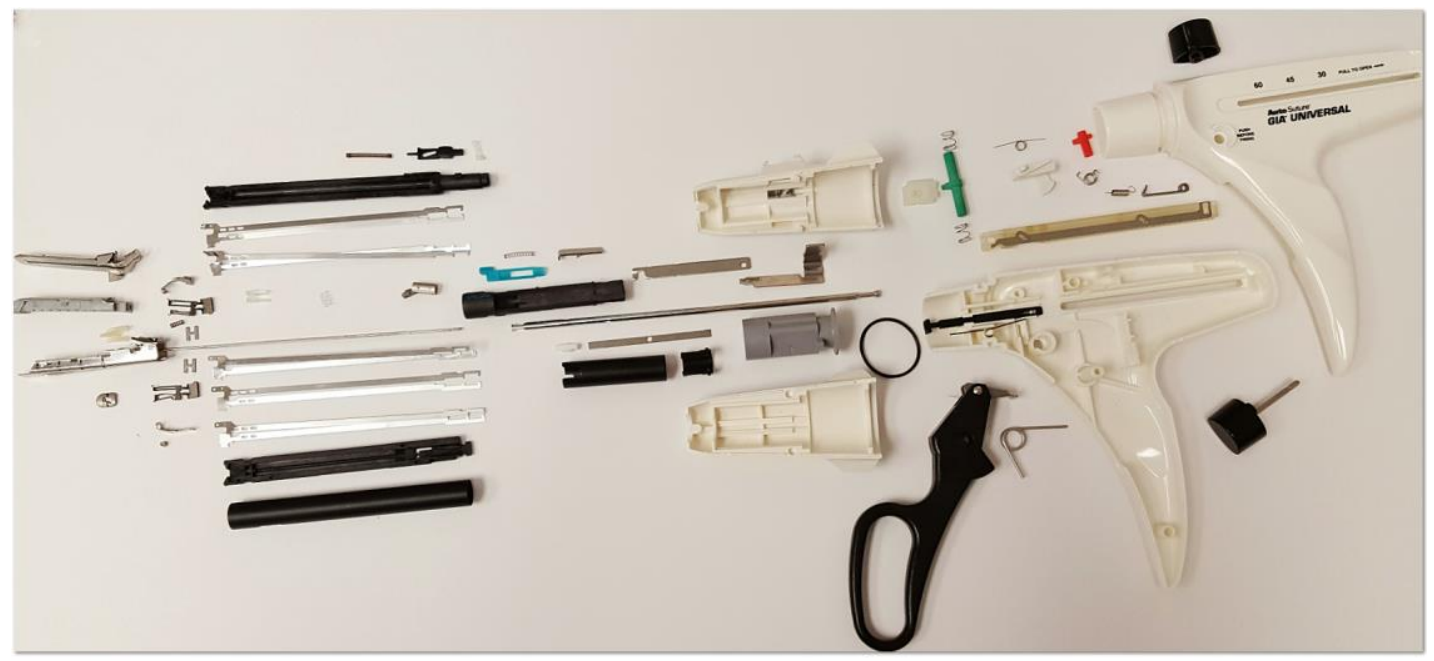

Figure 20. Covidien Endo GIA ${ }^{\mathrm{TM}}$ Universal stapler and the Covidien Endo GIA $^{\mathrm{TM}}$ Universal Roticulator ${ }^{\mathrm{TM}}$ reload exploded view.

A subset of the Ethicon ETS stapler DSM is shown in Figure 21. The full DSM is provided as an electronic attachment. 


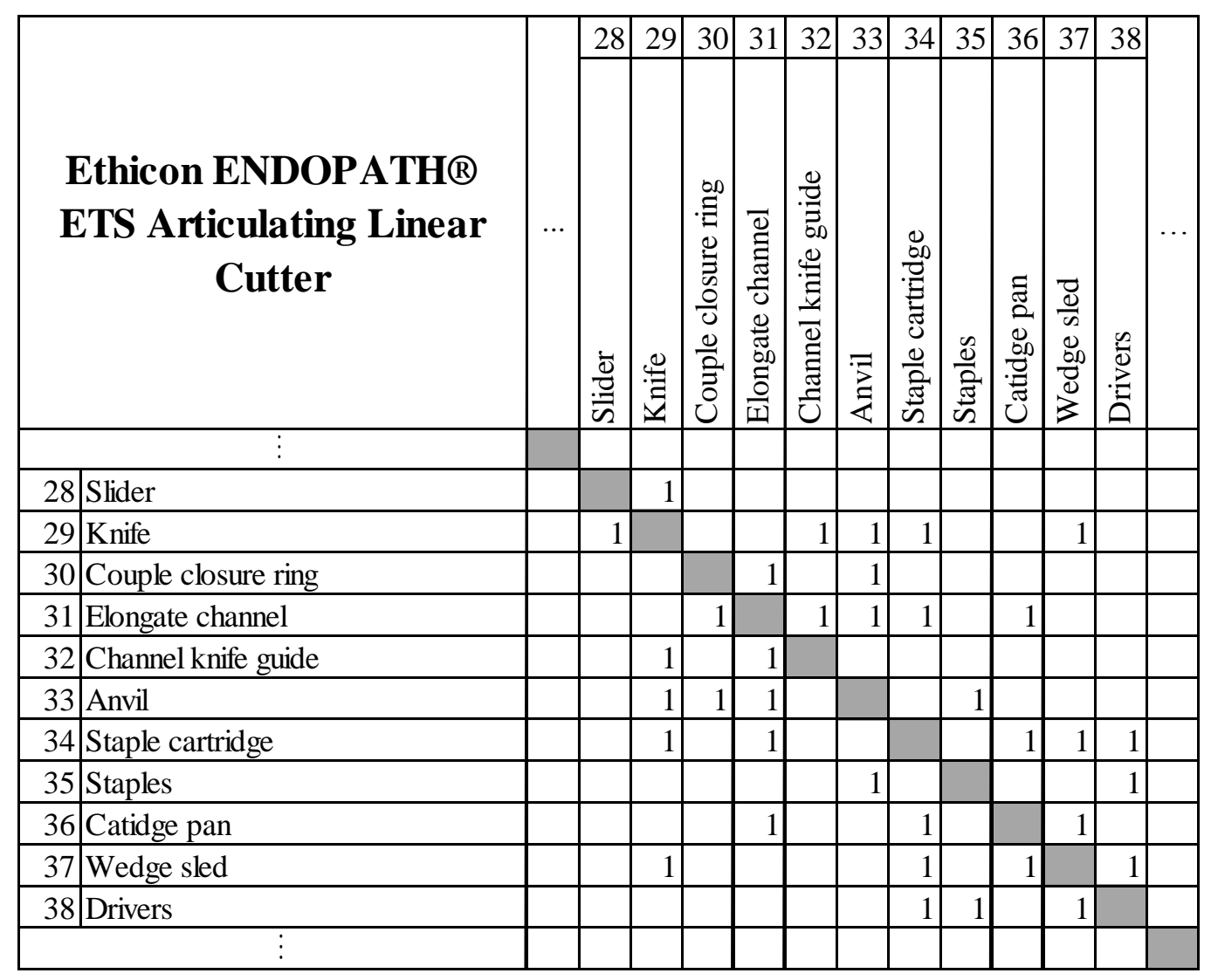

Figure 21. Ethicon ETS stapler DSM subset.

A subset of the Covidien Endo GIA stapler DSM is shown in Figure 22. The full DSM is provided as an electronic attachment. The Covidien Endo GIA consisted of the most components resulting in the largest DSM. 


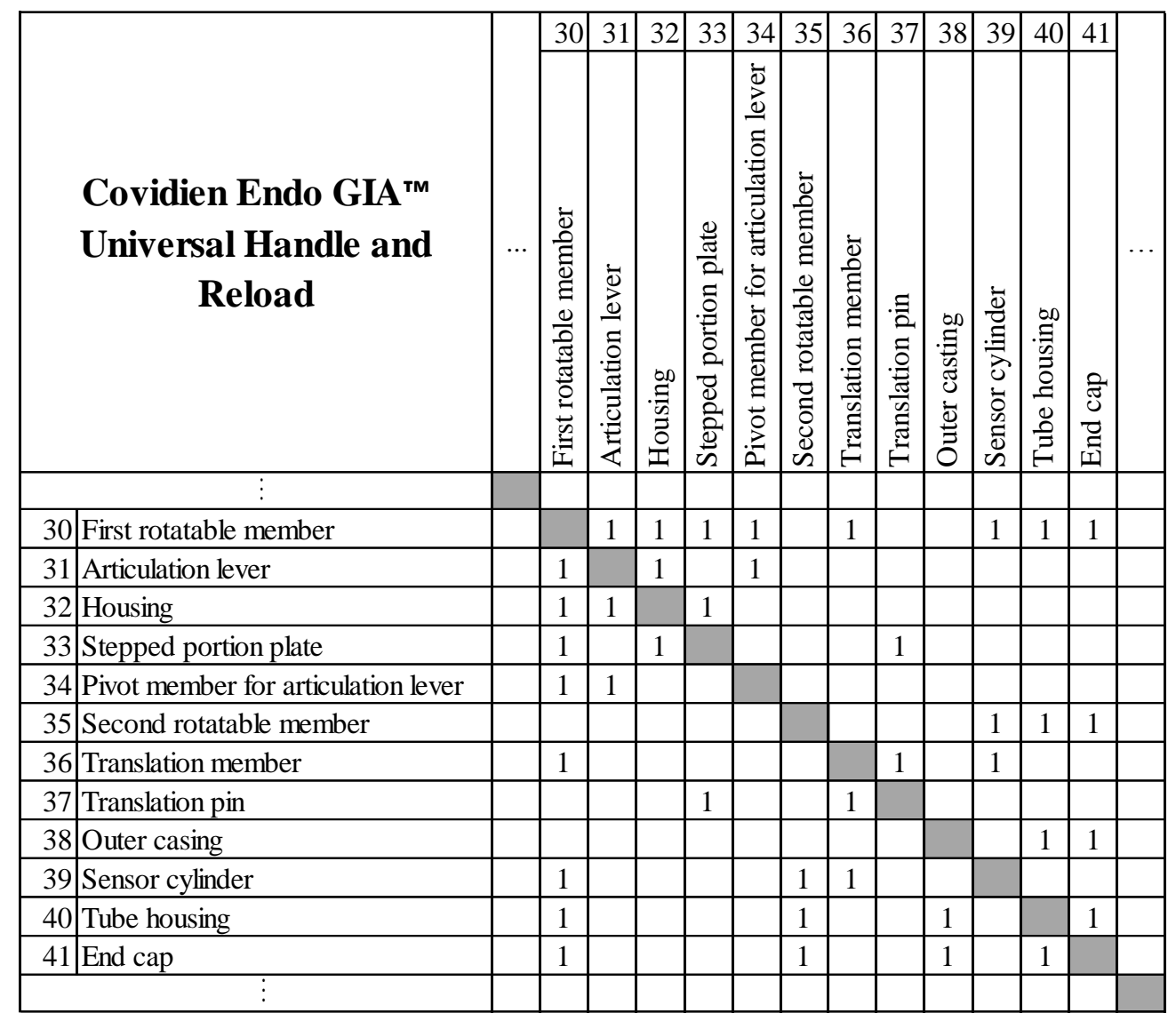

Figure 22. Covidien Endo GIA stapler DSM subset.

\subsubsection{Energy-Based Tissue Sealer Function and DSM}

The two brands of tissue sealers obtained for this analysis share a common main function, which involves sealing and transecting tissue by converting electrical energy into mechanical and thermal energy. However, the technology and mechanisms used to execute the functions differs. The Olympus Thunderbeat integrates both bipolar and ultrasonic technology in order to coagulate and dissect tissue. This device offers two modes that are put into effect via pressing the hand switches shown in Figure 23. In "Seal" mode, bipolar energy is delivered exclusively in order to create a seal without involving the cutting effects of ultrasonic vibrations. "Seal \& Cut" mode delivers a 
combination of bipolar and ultrasonic energy to seal and transect. The ultrasonic and bipolar probe tip of the jaw can be used to create a puncture in the tissue, and the jaw itself can be used as a grasper. Olympus offers three handle types for the Thunderbeat including a front-actuated grip, pistol grip, and inline grip. The device obtained for this analysis featured the pistol grip.

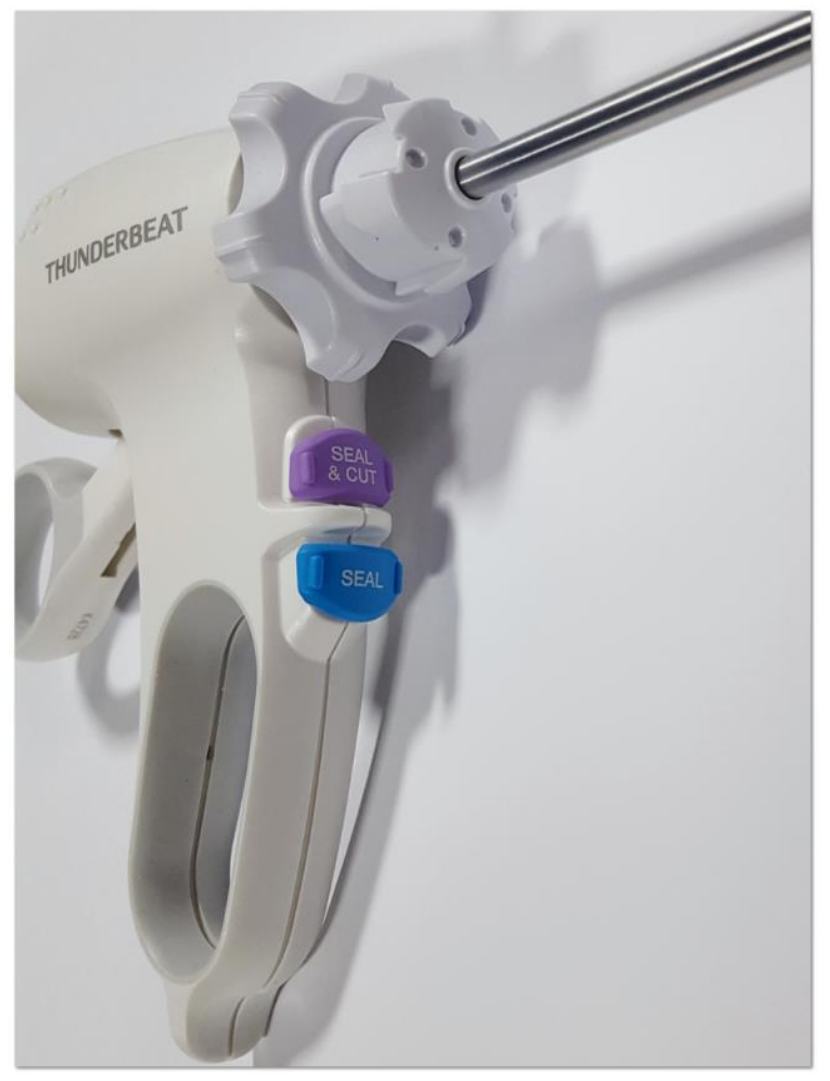

Figure 23. Olympus Thunderbeat activation hand switches.

The Ethicon Harmonic Ace utilizes ultrasonic energy, which involves the conversion of electrical energy to mechanical energy to thermal energy. However, ultrasonic devices do not pass current through tissue to achieve the desired effects. The 
Harmonic Ace assessed uses a pistol grip handle and delivers either "Min" or "Max" power when activated (see Figure 24).

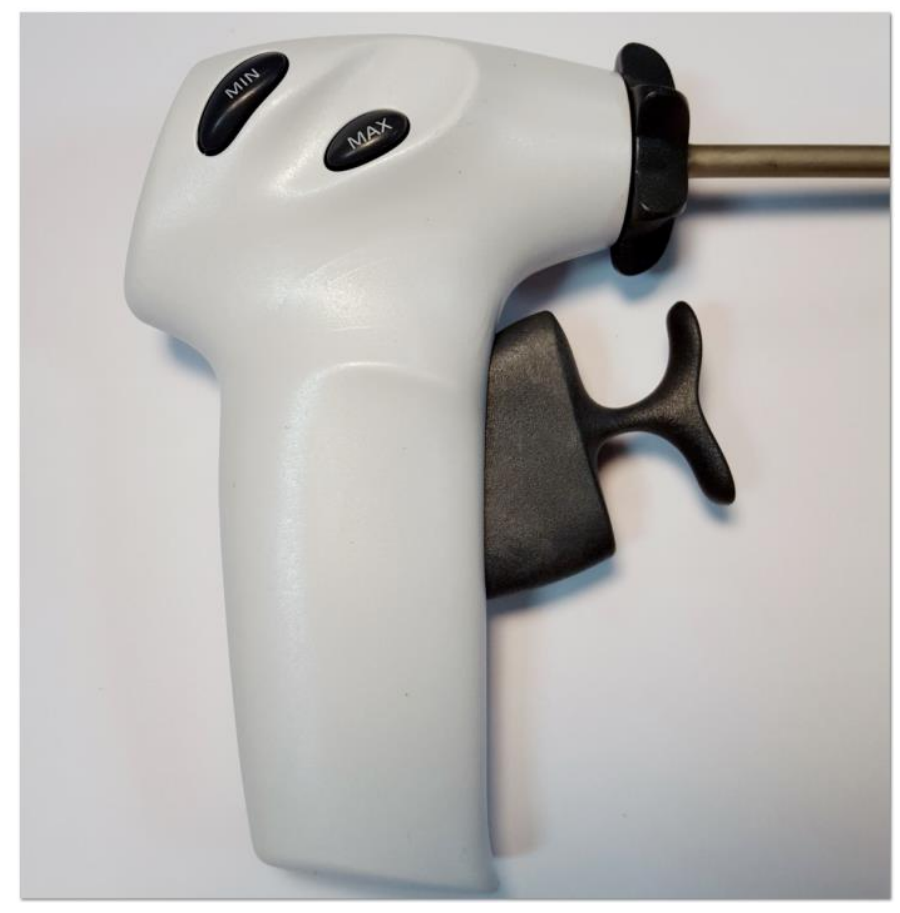

Figure 24. Ethicon Harmonic Ace activation hand switches.

Despite the differences in sealing and cutting technology, the devices share common features. Both devices require a hand piece attachment and connection to an electrosurgical generator in order to access, deliver, and convert electrical energy. A torque wrench is provided with each device to attach and detach the hand piece. The hand pieces contain piezoelectric transducers and convert the drive current into the ultrasonic output (Van Slycke, Gillardin et al. 2016). The jaw of each device can be used to grasp tissue, and the active blade of the Harmonic Ace and probe tip of the Thunderbeat can both be used to define tissue planes (Ethicon 2017, Olympus 2017). The jaw features of each device are shown in Figures 25 and 26, respectively. 


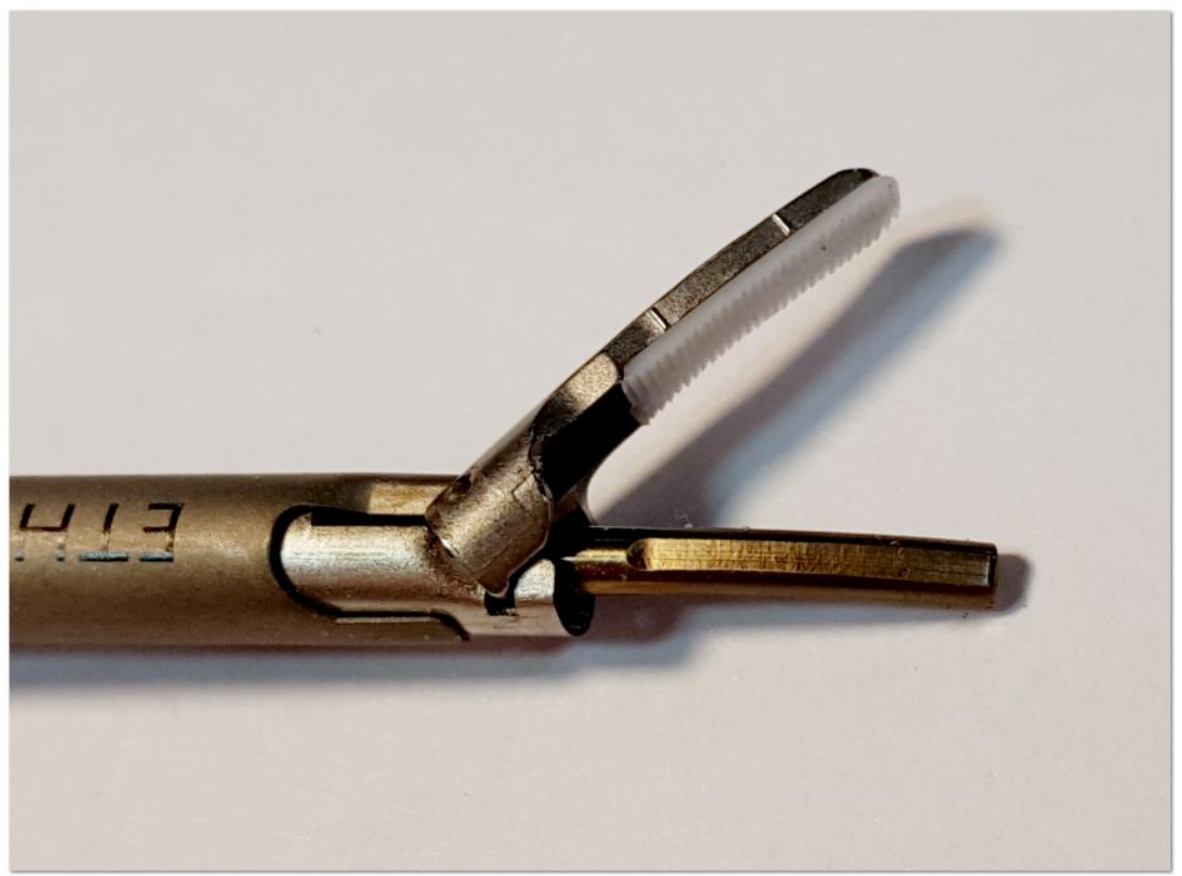

Figure 25. Ethicon Harmonic Ace ultrasonic jaw (active blade).

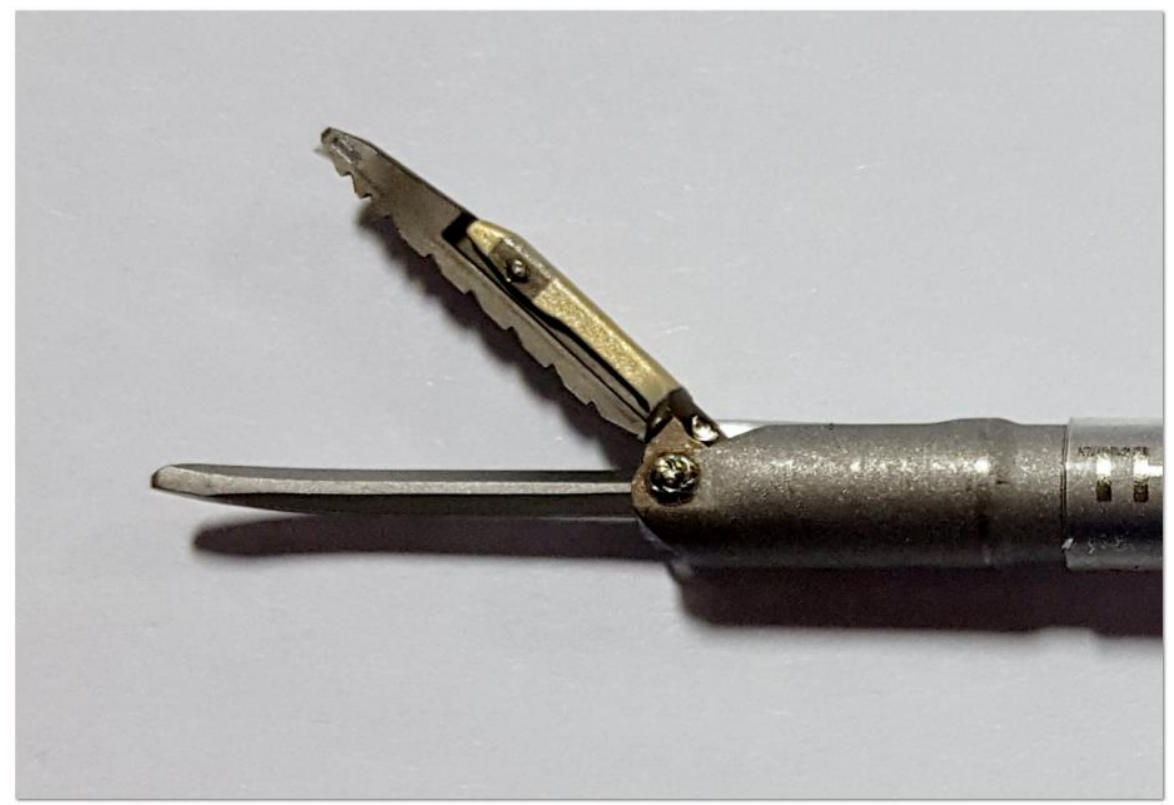

Figure 26. Olympus Thunderbeat ultrasonic and bipolar jaw (active probe tip). 
Each shaft can be rotated via a knob located near the proximal end of the shaft;

this allows for simple and efficient rotation of the jaw via the users' fingers while maintaining a secure grip on the device. Additionally, the general function of the devices are the same as shown in the functional model in Figure 27. The cutting and sealing functions of a tissue sealer is represented with the following functional model terminology: "separate" and "secure" tissue. The functional model provides a general overview of the types of energy employed by tissue sealers (Ethicon 2017, Olympus 2017).

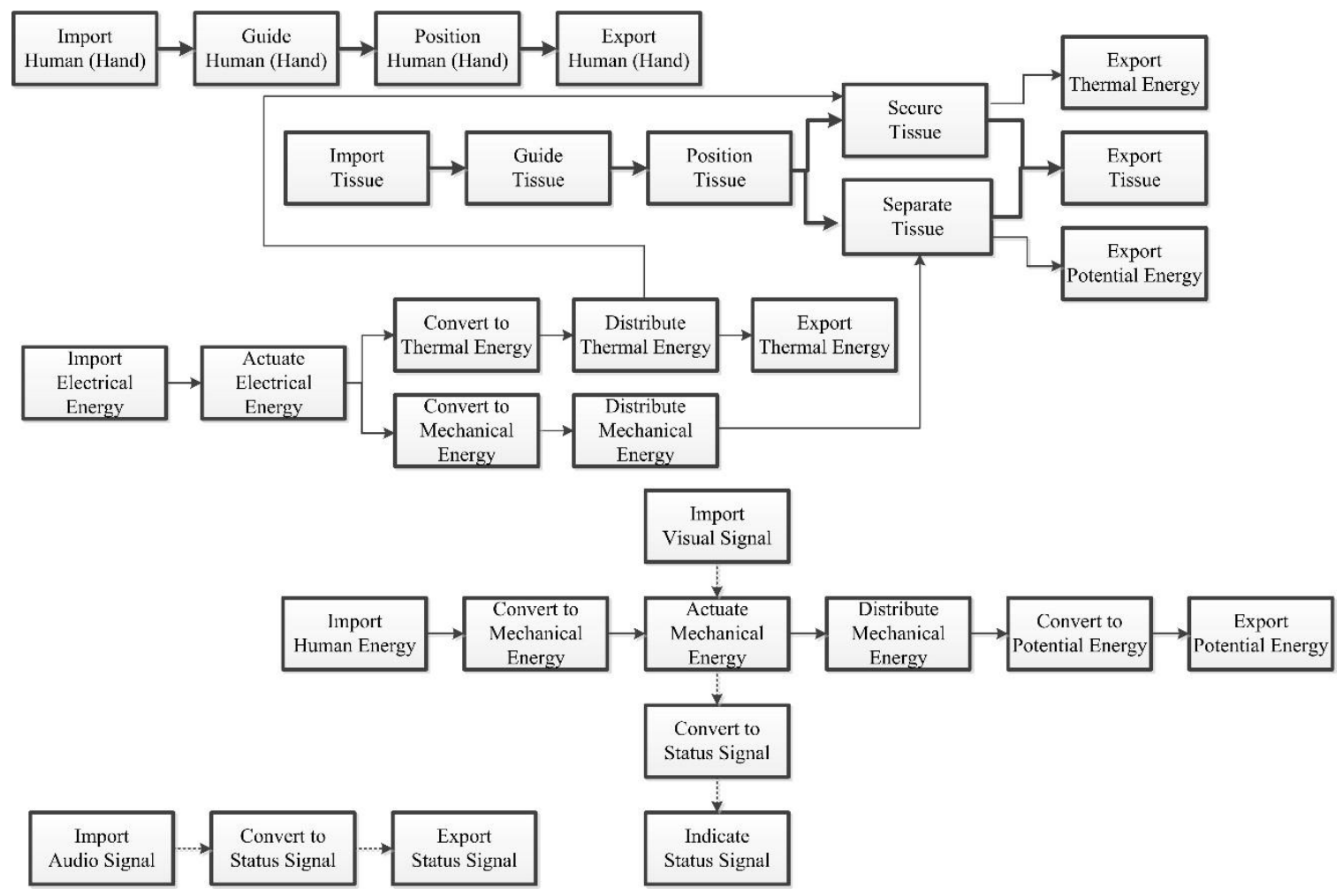

Figure 27. Energy-based tissue sealer functional model.

The conversions specific to different brands and types of tissue sealing devices may vary slightly from this functional model, but the main feature involving utilization of 
electrical energy is depicted. The DSM derived from the functional model is included as an electronic attachment. The devices were reverse engineered to assess connectivity. Exploded views of the Olympus Thunderbeat and Ethicon Harmonic Ace components are shown in Figures 28 and 29, respectively.

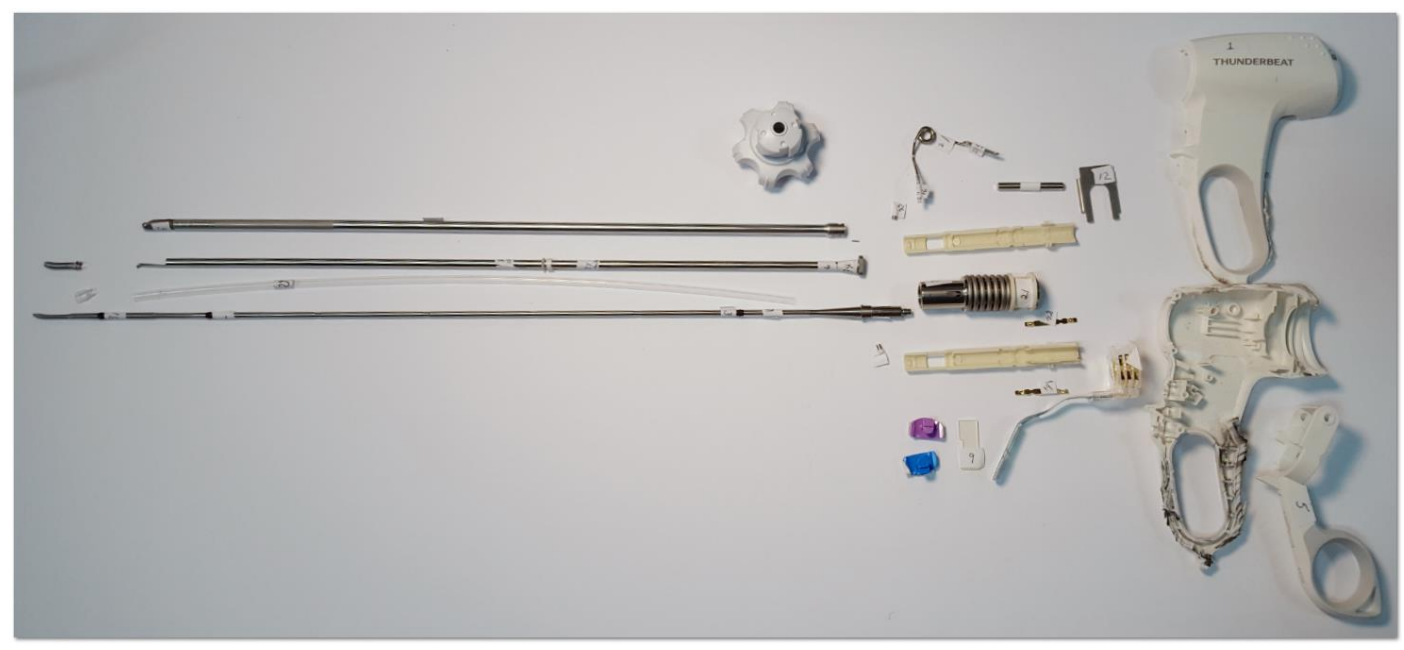

Figure 28. Olympus Thunderbeat exploded view.

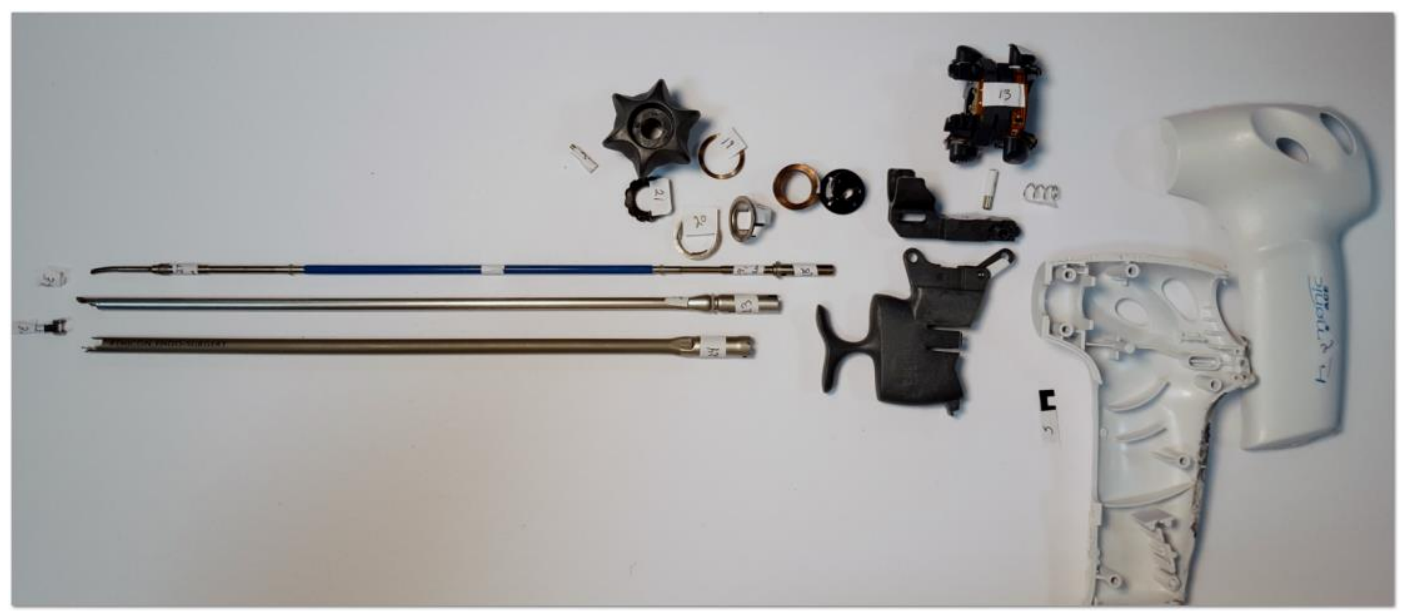

Figure 29. Ethicon Harmonic Ace exploded view.

A subset of the Olympus Thunderbeat DSM is shown in Figure 30. The full DSM is included as an electronic attachment. 


\begin{tabular}{|c|c|c|c|c|c|c|c|c|c|c|c|}
\hline & 27 & 28 & 29 & 30 & 31 & 32 & 33 & 34 & 35 & 36 & 37 \\
\hline $\begin{array}{c}\text { Olympus Thunderbeat } \\
\text { Surgical Tissue Management } \\
\text { System }\end{array}$ & 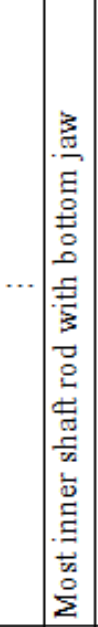 & 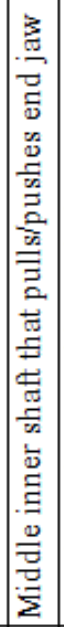 & 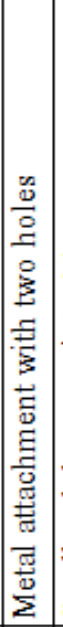 & 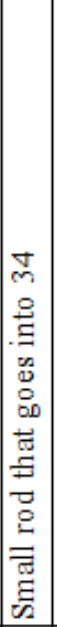 & 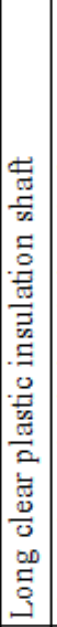 & 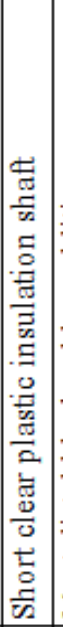 & 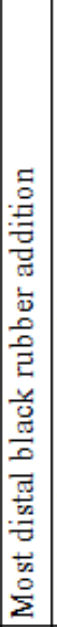 & 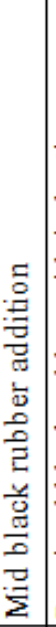 & 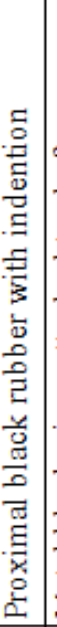 & 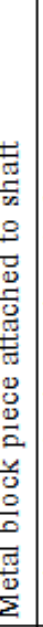 & 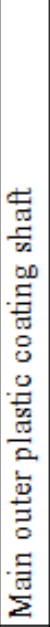 \\
\hline $\bar{\vdots}$ & & & & & & & & & & & \\
\hline \begin{tabular}{l|l}
27 & Most inner shaft rod with bottom jaw \\
\end{tabular} & & & & & 1 & 1 & 1 & 1 & 1 & & \\
\hline \begin{tabular}{l|l}
28 & Middle inner shaft that pulls/pushes end jaw \\
\end{tabular} & & & & 1 & 1 & 1 & & & 1 & & \\
\hline 29 Metal attachment with two holes & & & & & & & & & & & \\
\hline \begin{tabular}{|l|l|}
30 & Small rod that goes into 34 \\
\end{tabular} & & 1 & & & & & & & & & \\
\hline 31 Long clear plastic insulation shaft & 1 & 1 & & & & & 1 & 1 & 1 & & \\
\hline \begin{tabular}{|l|l|}
32 & Short clear plastic insulation shaft \\
\end{tabular} & 1 & 1 & & & & & & & 1 & & \\
\hline \begin{tabular}{|l|l|}
33 & Most distal black rubber addition \\
\end{tabular} & 1 & & & & 1 & & & & & & \\
\hline 34 Mid black rubber addition & 1 & & & & 1 & & & & & & \\
\hline \begin{tabular}{l|l}
35 & Proximal black rubber with indention \\
\end{tabular} & 1 & 1 & & & 1 & 1 & & & & & \\
\hline 36 Metal block piece attached to shaft & & & & & & & & & & & \\
\hline \begin{tabular}{l|l}
37 & Main outer plastic coating shaft \\
\end{tabular} & & & & & & & & & & & \\
\hline
\end{tabular}

Figure 30. Olympus Thunderbeat DSM subset.

A subset of the Ethicon Harmonic Ace DSM is shown in Figure 31. The full DSM is included as an electronic attachment. 


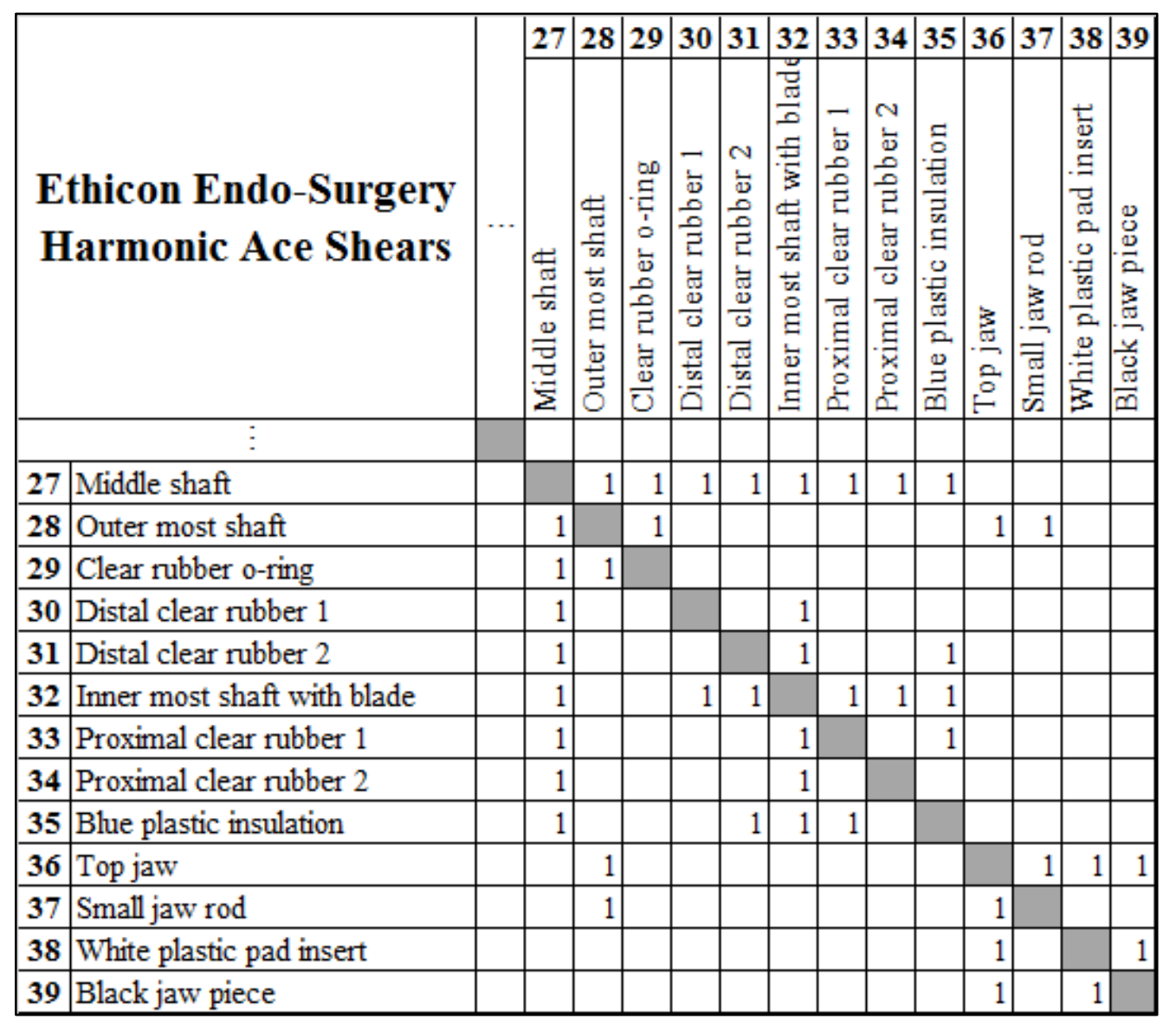

Figure 31. Ethicon Harmonic Ace DSM subset.

The full DSMs were utilized in the complexity analysis as a continuous matrix. Relative to the endoscopic staplers, patents containing the proper part descriptions were not as readily available via general patent searches. Therefore, the part names used in the tissue sealer DSMs are indicative of the part descriptions to assist identification versus adherent to manufacturers' naming conventions.

\subsubsection{Complexity Scores}


The complexity scores were based on the connectivity of the components or, in other words, the decomposability of the devices. The number of components and complexity scores for each device are shown in Table 40. The devices are arranged from lowest to highest complexity score.

Table 40. Number of components and complexity scores per device.

\begin{tabular}{|c|c|c|}
\hline Device & Number of Components & Complexity Score \\
\hline Ethicon Harmonic Ace & 39 & 170 \\
\hline Ethicon ETS & 38 & 184 \\
\hline Olympus Thunderbeat & 48 & 214 \\
\hline Covidien Endo GIA & 89 & 478 \\
\hline
\end{tabular}

Complexity scores were also calculated for each functional model based on the connectivity between the functions. The number of functions and the complexity scores for endoscopic stapler and tissue sealer functional models are shown in Table 41.

Table 41. Functional model parameters and complexity score.

\begin{tabular}{|c|c|c|}
\hline Functional Model & Number of Functions & Complexity Score \\
\hline Energy-based Tissue Sealer & 31 & 58 \\
\hline Endoscopic Stapler & 25 & 48 \\
\hline
\end{tabular}

The energy-based tissue sealer functional model contained more functions and was more complex than the endoscopic staplers. The value of creating a functional model from a general functional requirement is demonstrated by the two devices types as they show the various design paths that can be taken depending on the application. Figure 32 displays the branching that starts from the initial functional requirement to the 
mechanisms to satisfy the requirement and finally to designs and products that ultimately fulfill the goal of separating and securing tissue.

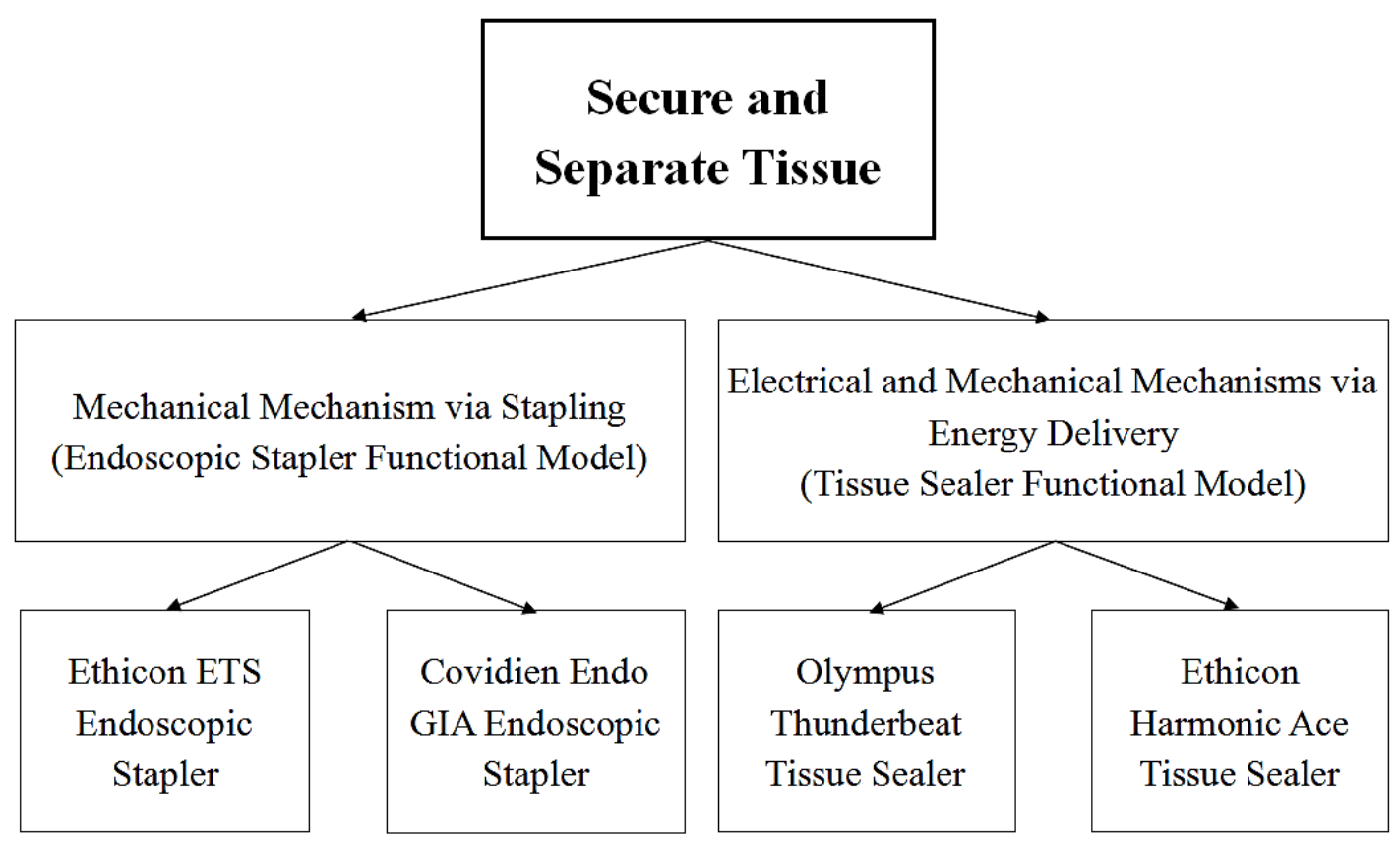

Figure 32. Branching of mechanisms and designs from functional requirements.

\section{$\underline{4.4 \text { Complexity Discussion }}$}

Complexity has been defined as "the measure of the degree of interconnections, the size of the composite, and how difficult it is to solve or analyze" (Summers and Shah 2010). The complexity scores calculated assess the degree of interconnectedness or decomposability. The complexity of a system or product increases as the decomposability score increases given that it is a measure of the requisite steps for structural disassembly (Mohinder, Gill et al. 2016). The Ethicon Harmonic Ace had the 
lowest complexity score (170), and the Covidien Endo GIA had the highest (478). The Ethicon ETS and Covidien Endo GIA consisted of the lowest and highest number of components, respectively (ETS - 38, Endo GIA - 89). When comparing the number of components and complexity scores per device type, a higher complexity score correlates to a higher number of components. However, this relationship only exists when assessing the values for endoscopic staplers and tissue sealers separately. The same relationship does not exist when assessing the values for all four devices as a single data set.

The connectivity algorithm is a systematic approach that considers the coupling between elements. When assessing the complexity of design products, a lower level of structural complexity is more favorable given that a design that involves less coupling enables mass customization, serviceability, and reusability (Ameri, Summers et al. 2008, Summers and Ameri 2008). This view of complexity considers the physical attachment of the various components that make up a product. Summers and Shah state that considering independent complexity assessments is valuable in industry as it pertains to "correlating manufacturing and life-cycle costs with early-stage and mid-stage design" (Summers and Shah 2010). Therefore, it can be inferred that from a structural perspective involving coupling complexity the medical devices with a lower complexity score embody a more simplistic and desirable design as it relates to the design process and product lifecycle.

The Covidien Endo GIA had the highest complexity based on this perspective (478), which is intuitive given the number of components and the modular design features required between the handle and the reload. The handle must facilitate 
articulation of the device jaw and deployment of stapling and cutting mechanisms. Various components and inherent connections are required for each of these functions. The reload is attached as an extended shaft potion. This attachment must bridge the components between the handle portion of the shaft and the reload portion of the shaft in order to seamlessly execute the required tasks. Conversely, the Ethicon ETS shaft and the components connecting the handle mechanism to the device jaw are continuous.

The Covidien Endo GIA contains a "break" in components of the shaft, which inevitably increases the part count, connections required, and complexity score. It is said that a good design is one that "satisfies all functional requirements with minimum number of components and relations" (Suh 2001). The Ethicon ETS and Covidien Endo GIA both satisfy the functional requirements to secure and separate tissue via mechanical mechanisms, yet the inner workings to execute the functions vary. Despite the increase in complexity score likely caused by the modular features of the Covidien Endo GIA, these same features may be viewed as beneficial by certain users. The modular features allow the user to introduce a new knife, jaw, and distal articulation assembly each time the function is carried out during the same procedure. New components for each firing can be viewed as a positive feature from a surgeons' perspective to alleviate any worries regarding dulling of the knife or damage to reload components that may have occurred during previous firings. However, from a complexity standpoint this may not be ideal given that the introduction of new components each time may increase potential of failure, and, as mentioned, the modularity is a major contributor to the increase in the complexity score. 
Of the two tissue sealers, the Olympus Thunderbeat had a higher complexity score (Thunderbeat -214 , Harmonic ace -170 ) associated with a higher number of components (Thunderbeat - 48, Harmonic Ace - 39) relative to the Ethicon Harmonic Ace. The Thunderbeat must incorporate components and features that enable delivery of both bipolar and ultrasonic energy, which could be reason for a higher complexity score. The Harmonic Ace solely delivers ultrasonic energy and was shown to have the lowest complexity score of all devices analyzed. Due to the Thunderbeat's integration of two sealing technologies, it has been shown to be highly effective in its sealing capabilities, and its performance has been deemed equivalent at the least and even superior in some cases compared to that of similar energy-based tissue sealers (Milsom, Trencheva et al. 2012, Seehofer, Mogl et al. 2012, Aytan, Nazik et al. 2014, Liberman, Khereba et al. 2014, Obonna and Mishra 2014, Van Slycke, Gillardin et al. 2016). Recall typically a higher complexity is viewed as an unfavorable design attribute; however, that is debatable in this particular case. The Thunderbeat satisfies the same functional requirements as the Harmonic Ace and, in fact, has been shown to have the best-in-class performance per specific applications addressed in the scientific literature (Olympus 2012, Olympus 2012, Obonna and Mishra 2014) yet it has a higher complexity score and number of components.

It may be argued that additional complexity based on decomposability can be favorable contingent upon whether the additional features provide superior results relative to that of a less complex device. Though, decomposability directly relates to the mechanical mechanisms inherent to the devices while the electrical features play a major role in tissue sealers' performance. Further investigation into how electrical energy can 
be accounted for in current complexity measures is necessary prior to concluding on this matter, especially as it relates to the electrical parameters that attribute to device performance. Performance contributions inherent to an increase in design complexity should be carefully weighed considering an increase in the difficulty of disassembly of the Thunderbeat inherent to supplying two types of sealing technologies has been shown to be beneficial in application.

While assessing complexity based on connectivity provides value and objective insight that aid design decisions (Ameri, Summers et al. 2008, Mohinder, Gill et al. 2016), it excludes aspects of a device outside of the mechanical mechanisms that dominate the connectivity. Tissue sealers require an electrical connection to an electrosurgical generator. The electrical features of these devices are necessary to carry out the function, yet it can also be responsible for device failures. The electrical mechanisms, hand piece, and generator required are not considered in the connectivity algorithm, but they may contribute to the complexity of a device. As discussed, there are many different approaches to assessing device complexity and it should not be viewed singularly (Summers and Ameri 2008). Complexity should be viewed from a structural, functional, and behavioral perspective (Ameri, Summers et al. 2008). The connectivity algorithm provides a solid base for assessing the complexity of the devices and allows a direct comparison between the devices based on decomposability and component connectivity alone.

The application of complexity extends beyond the product design itself and product lifecycle. The graph connectivity method has been investigated as a method to predict information from design representations (i.e. function structures and assembly 
times) (Mohinder, Gill et al. 2016). Mohinder et al. concluded these design representations can be used to predict product assembly time and market value; however, this information is more applicable to the early stages of design in order to facilitate more informed design decisions (Mohinder, Gill et al. 2016). The developers of the connectivity algorithm emphasize the necessity to aggregate various properties in their approach, which includes identifying metrics for size, interconnectivity, centrality, and decomposition. This research investigates decomposition and provides a foundation for demonstrating its potential value in the medical device industry. The work herein utilizes the foundation by assessing association between complexity and the severity of adverse event outcomes, yet the application of this calculation extends beyond the scope of this work.

While the data does not indicate one device type as overall more complex than the other, there are important aspects of each to consider as it pertains to this analysis. Endoscopic stapler reloads contain multiple staples, and the staples were treated as one component in the DSMs created in this study. The number of staples per reload can vary based on the type of tissue it is intended for. The particular reloads for the Ethicon ETS and Covidien Endo GIA acquired each deploy two parallel rows of 24 staples on either side of the cut making 48 staples total. If each staple had been treated as an individual component connecting to the cartridge, sled, and driver, then the total components and connectivity would significantly increase. Given each endoscopic stapler contained the same numer of staples in the reload, it would not affect the complexity score difference between the Ethicon ETS and the Covidien Endo GIA; however, it would result in higher 
complexity scores relative to both tissue sealers. Each staple has the potential to fail and may need to be analyzed individually when assessing complexity in future analyses.

The number of functions and a complexity score were derived from each device functional model. The tissue sealer functional model consisted of more functions relative to the endoscopic stapler in order to achieve the main functions of securing and separating tissue (Tissue Sealer - 31, Endoscopic Stapler - 25). Further, the tissue sealer functional model also resulted in a higher complexity score based on the connectivity algorithm (Tissue Sealer - 58, Endoscopic Stapler - 48). Therefore, the tissue sealer is more functionally complex than the endoscopic stapler based on these models which is likely due to the differences in mechanisms and operation. The particular endoscopic staplers analyzed herein deploy strictly mechanical mechanisms in order to execute the main functions involving stapling and cutting tissue. However, the tissue sealers require an input of electrical energy and conversion into mechanical and thermal energy in order to carry out the main task.

Function is crucial to consider when assessing potential for product failure and failure scenarios and is most beneficial when considered early in the conceptual phases of the design process. A relationship has been demonstrated between product function and risk likelihood and consequence (Tumer and Stone 2003, Lough, Stone et al. 2008). The consideration of function has been deemed useful in particular for the Function-Failure and Risk in Early Design (RED) methods (Lough, Stone et al. 2008).

It is important to reiterate that despite the similarity in the main functional requirement of securing and separating tissue, the two devices types are typically used for different types of procedures and tissue types. Tissue sealers, such as the Thunderbeat 
and Harmonic Ace, can be used to seal and cut vessels that are up to between $5-7 \mathrm{~mm}$ in size (Ethicon 2017, Olympus 2017), whereas the endoscopic staplers can be applied on relatively larger tissue types such as lung and bowel tissue. An endoscopic stapler is not meant for dissecting small vessels just as a tissue sealer (indicated for up to $5 \mathrm{~mm}$ or $7 \mathrm{~mm}$ vessels) is not suited for removing large portions of the stomach. Therefore, while they both are well suited for laparoscopic surgeries and MIS, their specific applications must be well understood and considered when assessing design and functional model complexity. The four devices demonstrate the important role functional modeling can play in brainstorming various mechanisms and design approaches to fulfill the same functional requirement depending on the application.

\subsection{Complexity Study Limitations and Future Work}

The limitations to consider relate to the connectivity algorithm and the specific devices chosen for the analysis. The connectivity algorithm produces a complexity score based on decomposability and does not take into account the electrical features of the tissue sealers. It is recommended to assess the contribution of the electrical features to the complexity measure and, if necessary, how it could be incorporated into a final complexity score for a medical device. Complexity of the medical devices must be viewed from various perspectives as discussed in the scientific literature (Hölttä and Otto 2005, Ameri, Summers et al. 2008, Hagedorn, Grosse et al. 2015). Additionally, the staples of the reloads were considered one part in the DSMs entered in the connectivity 
algorithm code. It may be necessary to consider each staple as an individual part in future analysis considering each staple has the potential to fail.

The methods outlined for determining complexity should be implemented in the early stages of design. The approach utilized assessed the complexity of a final product. If the various perspectives of complexity are considered at the very early stages in conceptual design, the comparisons between features (as it relates to concepts, designs, components, assembly, etc.) would be more valuable given the flexibility at these steps. This also provides the opportunity to assess potential failure prior to the finalization of design and manufacture of a product. Both structural and functional complexity are more useful if applied earlier; though this work demonstrated the application of complexity to current products and its potential uses and feasibility.

Additional devices and device classes should be investigated when measuring and comparing complexity. Herein, two staplers were reverse engineered that operate via mechanical mechanisms to carry out the same function. However, the two tissue sealers investigated are not identical in the mechanisms deployed to carry out the function. While they both utilize ultrasonic energy, the Thunderbeat also deploys bipolar energy and was the only device offering this combination during the study period. Given this was a relatively recent innovation, it was deemed important to include. However, for complexity score comparison it would also be beneficial to compare tissue sealers that utilize the exact same energy deliver methods, such as comparing the Ethicon Harmonic Ace to the Covidien Sonicision ${ }^{\mathrm{TM}}$. The number and types of devices chosen for this analysis were limited by the resources available to acquire such types of products. 
Future work should also involve mapping the components to the specific function(s) they carry out. Such mapping would assist the tracing of historical failure modes; however, this technique is best utilized early in the design process (Stock, Stone et al. 2003, Stone, Tumer et al. 2004). 


\section{CHAPTER V}

\section{OUTCOME SEVERITY AND DEVICE DESIGN COMPLEXITY}

\section{$\underline{5.1 \text { Outcome Severity and Design Complexity Study Overview }}$}

Users and manufacturers of minimally invasive surgical devices require a comprehensive understanding of the factors that potentially affect device performance, failure modes, and the severity of adverse event outcomes. Currently there are few methods to integrate knowledge of clinical outcomes and device use with the engineering design process in order to investigate related factors. A new approach was presented to integrate the clinical and design domains via assessing severity of adverse event outcomes and the complexity of the associated device design. The approach provides a foundation for aiding in prevention of adverse clinical outcomes and bridging the gap between device users and designers.

The overall goal of Specific Aim 5 was to describe the relationship between minimally invasive surgical device design complexity and the severity of adverse event outcomes associated with endoscopic staplers and energy-based tissue sealers, which is carried out in this chapter. It was hypothesized that as minimally invasive surgical device design complexity increases, the severity of adverse event outcomes associated with the device also increases. In addition, the goal of Specific Aim 5 was to develop a tool to 
predict the likelihood of severe adverse events associated with minimally invasive surgical devices based on design complexity.

\section{$\underline{5.2 \text { Severity and Complexity Methods }}$}

The goal of determining the relationship between outcome severity and device design complexity based on connectivity and an outcome severity predictive tool based on the complexity was explored by performing ordinal regression. The outcome severity assigned to each adverse event report in Chapter III was grouped per device and corresponding complexity score from Chapter IV. The severity of the adverse event outcomes was the ordinal, dependent variable. The independent design complexity score should be deemed a continuous variable; however, due to the lack of devices obtained and corresponding complexity scores calculated, not enough samples were collected to classify complexity as a continuous variable for a robust statistical output (i.e. the ordinal model failed to improve indicating a poor fit and resulted in a very low standard error). In order for complexity to be assessed statistically as a continuous variable, more data points and corresponding standard deviations are necessary. Therefore, the ordinal severity data was grouped under categorical data consisting of each complexity score.

The average severity of the outcomes was reported for each design and functional model complexity score. The total sample of severity data included outcomes severities from 383 reports. 100 severity outcomes were matched to each complexity score except for the Thunderbeat device. The severities assigned to the total population of reports (83) were matched to the complexity score for the Thunderbeat device. By default, the 
statistical software SPSS used the highest value as the reference variable for ordinal regression. The parameter estimates from the output were used to calculate the odds ratios and to assess the statistical significance of the effect with respect to each complexity value $(p=0.05)$. The odds ratios were calculated by taking the exponential of the estimates.

\section{$\underline{5.3 \text { Severity and Complexity Results }}$}

Sample information for the dependent variable, severity, and the independent variable, complexity, is shown in Table 42.

Table 42. Sample percentages for regression variables.

\begin{tabular}{|l|l|c|c|}
\hline \multicolumn{2}{|c|}{} & N & $\begin{array}{c}\text { Marginal } \\
\text { Percentage }\end{array}$ \\
\hline \multirow{4}{*}{ Severity } & 1.00 & 37 & $9.7 \%$ \\
\cline { 2 - 4 } & 2.00 & 67 & $17.5 \%$ \\
\cline { 2 - 4 } & 3.00 & 205 & $53.5 \%$ \\
\cline { 2 - 4 } & 4.00 & 56 & $14.6 \%$ \\
\cline { 2 - 4 } & 5.00 & 18 & $4.7 \%$ \\
\hline \multirow{4}{*}{ Complexity } & 170.00 & 100 & $26.1 \%$ \\
\cline { 2 - 4 } & 184.00 & 100 & $26.1 \%$ \\
\cline { 2 - 4 } & 214.00 & 83 & $21.7 \%$ \\
\cline { 2 - 4 } & 478.00 & 100 & $26.1 \%$ \\
\hline Valid & 383 & $100.0 \%$ \\
\hline Missing & 0 & \\
\hline \multicolumn{1}{|c|}{ Total } & 383 & \\
\hline
\end{tabular}

The overall average severity was 2.87 . The average severity per complexity score is shown in Table 43 in addition to the device type. 
Table 43. Design complexity scores and average severities per device type.

\begin{tabular}{|c|c|c|}
\hline Device Type & Complexity Score & Average Severity \\
\hline Tissue Sealer & 170 & 2.65 \\
\hline Endoscopic Stapler & 184 & 3.17 \\
\hline Tissue Sealer & 214 & 2.63 \\
\hline Endoscopic Stapler & 478 & 2.99 \\
\hline
\end{tabular}

The average severity per device type and corresponding complexity score of the functional model is shown in Table 44.

Table 44. Functional model complexity scores and average severities per device type.

\begin{tabular}{|c|c|c|}
\hline Device Type & $\begin{array}{c}\text { Functional Model } \\
\text { Complexity Score }\end{array}$ & Average Severity \\
\hline Tissue Sealer & 58 & 2.64 \\
\hline Endoscopic Stapler & 48 & 3.08 \\
\hline
\end{tabular}

An ordinal regression was performed in SPSS statistical software to determine whether the complexity score had a statistically significant effect on outcome severity. The model fitting information is shown in Table 45. The abbreviation " $d f$ " stands for degrees of freedom.

Table 45. Model fitting information for ordinal regression.

\begin{tabular}{|c|c|c|c|c|}
\hline Model & $\begin{array}{c}-2 \text { Log } \\
\text { Likelihood }\end{array}$ & $\begin{array}{c}\text { Chi- } \\
\text { Square }\end{array}$ & df & P-value \\
\hline Intercept Only & 140.889 & & & \\
\hline Final & 118.445 & 22.444 & 3 & $<0.01$ \\
\hline
\end{tabular}


The model improved the ability to predict the severity of the outcome based on complexity. Though, the test of parallel lines was statistically significant $(p<0.05)$ indicating the odds were different between thresholds. The parameter estimates are shown in Table 46. The estimates were in reference to the severity associated with a complexity of 478 (Covidien Endo GIA complexity score).

Table 46. Ordinal regression parameter estimates with respect to a complexity score of 478.

\begin{tabular}{|c|l|c|c|c|c|c|}
\hline \multicolumn{2}{|l}{} & Estimate & Std. Error & Wald & df & P-value \\
\hline \multirow{4}{*}{ Threshold } & {$[$ Severity $=1.00]$} & -2.534 & 0.247 & 105.310 & 1 & $<0.01$ \\
\cline { 2 - 8 } & {$[$ Severity = 2.00] } & -1.233 & 0.206 & 35.679 & 1 & $<0.01$ \\
\cline { 2 - 8 } & {$[$ Severity $=3.00]$} & 1.292 & 0.208 & 38.668 & 1 & $<0.01$ \\
\cline { 2 - 8 } & {$[$ Severity =4.00] } & 2.886 & 0.292 & 97.512 & 1 & $<0.01$ \\
\hline \multirow{4}{*}{ Location } & {$[$ Complexity=170.00] } & -0.585 & 0.271 & 4.659 & 1 & 0.03 \\
\cline { 2 - 8 } & {$[$ Complexity=184.00] } & 0.371 & 0.271 & 1.873 & 1 & 0.17 \\
\cline { 2 - 8 } & {$[$ Complexity=214.00] } & -0.827 & 0.284 & 8.451 & 1 & $<0.01$ \\
\cline { 2 - 8 } & {$[$ Complexity=478.00] } & - & - & - & - & - \\
\hline
\end{tabular}

The odds ratio and significance of the effect with respect to each complexity score are shown in Table 47. 
Table 47. Outcome severity odds ratios and significance with respect to each complexity score.

\begin{tabular}{|c|c|c|}
\hline Complexity & Odds Ratio (e $\mathrm{e}^{\text {Estimate }}$ ) & Significance \\
\hline \multicolumn{3}{|c|}{ With Respect to Complexity $=478$} \\
\hline Complexity $=170$ & 0.56 & Significant \\
\hline Complexity $=184$ & 1.44 & Not Significant \\
\hline Complexity $=214$ & 0.44 & Significant \\
\hline \multicolumn{3}{|c|}{ With Respect to Complexity $=184$} \\
\hline Complexity $=214$ & 0.30 & Significant \\
\hline Complexity $=170$ & 0.38 & Significant \\
\hline Complexity $=478$ & 0.69 & Not Significant \\
\hline \multicolumn{3}{|c|}{ With Respect to Complexity = 214} \\
\hline Complexity $=478$ & 2.29 & Significant \\
\hline Complexity $=184$ & 3.31 & Significant \\
\hline Complexity $=170$ & 1.27 & Not Significant \\
\hline \multicolumn{3}{|c|}{ With Respect to Complexity $=170$} \\
\hline Complexity $=478$ & 1.79 & Significant \\
\hline Complexity $=184$ & 2.60 & Significant \\
\hline Complexity $=214$ & 0.79 & Not Significant \\
\hline
\end{tabular}

\subsection{Severity and Complexity Discussion}

The goal of this research was to determine the relationship between outcome severity and device design complexity based on connectivity. Based on this data sample and assessing the average severities per complexity score, a relationship was not shown to exist between the outcome severity and device design complexity. Specifically, the severity of adverse event outcomes did not increase as minimally invasive surgical device design complexity increased. Therefore, the null hypothesis stating there is no statistical significance between outcome severity and design complexity could not be rejected.

The majority of adverse events resulted in a severe outcome (53.5\%), and a moderate outcome was the second most common result (17.5\%). The average outcome 
severity was 2.87 , which is between a moderate and a severe outcome. The Ethicon ETS stapler had a complexity score of 184 and resulted in the highest average outcome severity of the adverse event reports assessed (3.17). This device resulted in the only average outcome severity above severity grade 3 . The second highest average outcome severity was 2.99 , which was associated with a complexity score of 478 and the Covidien Endo GIA stapler. The Covidien Endo GIA stapler resulted in the highest complexity score, but this device was not associated with the highest average outcome severity of the devices assessed.

The lowest average outcome severity was 2.63 and was associated with a complexity score of 214 and the Olympus Thunderbeat. The Thunderbeat was associated with the lowest average outcome severity, but this device did not result in the lowest complexity score. The lowest complexity score of 170 for the Ethicon Harmonic Ace resulted in an average outcome severity of 2.65. Despite the lack of evidence to show a relationship between the complexity scores and the average outcome severity of the associated adverse events, a trend existed in the outcomes severities across the device types. The endoscopic staplers assessed resulted in higher average outcome severities relative to that of the tissue sealers. Yet, the energy-based tissue sealer functional model contained more functions and was more complex than that of the endoscopic stapler.

The development of a predictive tool for outcome severity based on complexity was explored by performing ordinal regression. The Pearson and Deviance goodness-offit tests both indicated that the model was not a good fit to the observed data, $\chi^{2}(9)=$ 60.041 and $61.757, p<0.01$ and $p<0.01$. Further, the assumption of proportional odds was not met based on the test of parallel lines, $p<0.01$. However, the final model 
statistically predicted the dependent variable over and above the intercept-only model, $\chi^{2}(3)=22.444, p<0.01 .6 .2 \%$ of the variance was explained by the model per Nagelkerke Pseudo R-squared. Considering the violations, the findings should be interpreted with caution as the model may not provide a proper prediction of outcome severity.

Per the ordinal regression analysis, the odds ratios indicated that the severity associated with tissue sealer complexity scores 170 and 214 are likely to be lower than that of both endoscopic stapler complexity scores of 184 and 478 (odds ratios < 1), a statistically significant effect $(p<0.05)$. Conversely, the odds ratios indicated that the outcome severity associated with endoscopic stapler complexity scores 184 and 478 are likely to be higher than that of tissue sealer complexity scores 170 and 214 (odds ratios > 1), also a statistically significant effect $(p<0.05)$. The odds ratios predicting the outcome severities associated with tissue sealer complexity scores 170 and 214 relative to each other did not have a statistically significant effect $(p>0.05)$. Similarly, the odds ratios predicting the outcome severities associated with endoscopic stapler complexity scores 184 and 478 relative to each other did not have a statistically significant effect $(p>$ $0.05)$.

While a severity predictive tool could not be developed from this particular data set for complexity based on a continuous variable, the ordinal regression analysis provides relative insight for the outcome severities that result specifically in association with the devices and corresponding complexity scores assessed herein. Development of a robust predictive tool requires assessment of the severity of adverse event outcomes and design complexity scores of additional minimally invasive surgical devices. 
The integration of clinical and design based assessments should take into account the various approaches that are employed for analysis. Medical based studies utilize statistics in the research methods in order to draw accurate and unbiased inferences regarding the topic of study (Novack, Jotkowitz et al. 2006). A robust study design is mandatory in order to make proper conclusions regarding patient treatment, procedural outcomes, medical adverse events, etc. Conversely, various product design approaches utilize design heuristics in order to maximize creativity and diversity in design, promote variation in the concepts generated, and to provide alternate solutions to existing design problems (Yilmaz and Seifert 2011). The use of design heuristics assists the development of novel product concepts and design approaches (Yilmaz, Daly et al. 2016). Module heuristics in particular has been defined as "a method of examination in which the designer uses a set of steps, empirical in nature, yet proven scientifically valid, to identify modules in a design problem" (Stone, Wood et al. 2000). Therefore, heuristics can be viewed as an acceptable basis for assessing methodology particularly in the field of design.

A combination of statistical and design heuristic approaches was employed in order to investigate the relationship between design complexity and outcome severity. While statistical conclusions regarding the overall research goal could not be drawn from the analysis, heuristic methods support development of the approach presented. The work herein should be viewed as an essential step towards development and validation of a novel approach to assess the aspects of a device's design that may contribute to adverse minimally invasive device failure outcomes. 
This work provides one approach to quantifying complexity of minimally invasive surgical devices based on decomposability. However, confliction between the work system and the operator/user capabilities (or lack thereof) can also contribute to errors within complex systems (van Det, Meijerink et al. 2009). Dankelman and Grimbergen indicate five strategies aimed at the reduction of errors in minimally invasive surgery, one of which is to reduce complexity (Dankelman and Grimbergen 2005). Complexity in this context is relevant to the entire system of the device including its design, use, instructions, and procedural applications. The strategies are aimed at eliminations of unnecessary and inefficient interactions and processes, the standardization of procedures and equipment, and training (Flin, O'Connor et al. 2008).

The reduction of complexity in addition to implementing methods for the remaining strategies has been suggested to reduce minimally invasive surgical errors and, therefore, would likely mitigate the potential for severe adverse event outcomes. Provided the prevalence of both user and device-based failures outlined in Chapter II, a separate approach may be necessary targeted at reducing human errors. This approach would involve a system analysis to identify the root cause of user errors in addition to the implementation of defenses within the system to prevent adverse event outcomes from being induced by such errors (Dankelman and Grimbergen 2005).

The decomposability approach can aid identification of specific variables that contribute to design complexity, such as connectivity and number of components. Though, analysis of the adverse event reports indicates both user and device-based failures. Further sorting of the adverse events based on specific failure type may be valuable and more applicable to the calculation of complexity based on decomposability. 
For example, assessing the severity of outcomes that are explicitly stated to have occurred due to device failure may be more applicable to a complexity score based on the device design. Furthermore, the outcome severity of user-based failures may be more applicable to a score that assesses the elements of device instructions and operation and user experience and knowledge. Severity of outcomes should also be analyzed to determine association with procedure types that may affect aspects of device use, such as visibility, tissue type, application, etc.

\section{$\underline{5.5 \text { Severity and Complexity Study Limitations and Future Work }}$}

The limitations of this study are consistent with those of the outcome severity study and complexity study discussed in Section 3.5 and Section 4.5, respectively. Specific to this analysis, the assumptions of proportional odds was violated indicating the possibility that the ordinal regression odds ratios and corresponding conclusions are invalid. Complexity was not classified as a continuous variable and instead was classified as a categorical variable. Therefore, conclusions regarding outcome severity per this analysis must be made relative only to the individual complexity scores 170, 184, 214, and 478. Future work should include a power analysis to determine the proper sample size from which an appropriate budget can be determined to obtain more devices and investigate the feasibility of an outcome severity prediction tool based on design complexity. This study could serve as pilot data for a power analysis.

Development of a complexity-severity prediction tool should be investigated for its application in the early design stages to mitigate potential design-related adverse 
events. Design variables assessing complexity can potentially enhance engineers' abilities to prevent or reduce the severity of surgical device-based failure mode outcomes. Yet, a modified approach to assessing the relationship between outcome severity and device complexity is necessary.

The limitation specific to the calculation of endoscopic stapler complexity should be reiterated. In particular, the staples of each reload were considered as one part in the DSM and connectivity algorithm. Each staple has the potential to fail and, therefore, staples may need to be considered as individual parts. The inclusion of each staple separately would increase the part count and complexity score of each endoscopic stapler.

Complexity was one variable related to the use of minimally invasive surgical devices that was considered in the investigation of the severity of adverse event outcomes. While the analysis herein did not prove the existence of a relationship between outcome severity and device design complexity, there are additional factors to consider. Various methods for calculating complexity exist, and the method used herein utilized a decomposability score. As discussed in Section 5.4, investigation into operational and procedural factors that contribute to user-based errors, failures, and severe outcomes specifically could be beneficial while separately assessing design complexity in relation to device-based failures and the corresponding adverse event outcomes. 


\section{CHAPTER VI \\ SUMMARY AND CONCLUSIONS}

The overall goals of this research were to 1) characterize the design attributes, failure modes, and adverse events associated with minimally invasive surgical devices and 2) describe the relationship between minimally invasive surgical device design complexity and the severity of adverse events. A novel approach for investigating and evaluating minimally invasive device failures was presented, which involved assessing the severity of adverse event outcomes associated with the failures modes and exploring aspects of the devices' design that may contribute to failure. The approach was unique in that medical device design, function, and failure outcomes were evaluated from a complexity perspective.

Two endoscopic staplers, the Ethicon ETS and Covidien Endo GIA, were utilized in addition to two energy-based tissue sealers, the Olympus Thunderbeat and Ethicon Harmonic Ace. The failures modes, severity of adverse event outcomes, and complexity associated with these four devices were identified. The failures modes were derived from adverse event reports retrieved from the FDA MAUDE database. Severity grades were applied to each adverse event outcome associated with the failure modes via application of the CTCAE v4.0 grading scale. The connectivity algorithm was applied in order to generate a complexity score for each device based on decomposability. Finally, the 
overall outcome severity was explored for each device and corresponding complexity score by performing ordinal regression.

The most common failure modes were assessed for both device types in Chapter II. Failures associated with the endoscopic staplers occurred most frequently during the firing phase (ETS - 54.1\%, Endo GIA - 51.3\%). Overall, malformed staples or a failure to form staples contributed to the most adverse events that occurred during endoscopic stapler firing (ETS - 15.3\%, Endo GIA - 26.5\%). Failures associated with tissue sealers occurred most frequently during the activation phase of operation (Thunderbeat $-43.4 \%$, Harmonic Ace $-46.1 \%$ ). The top two failures modes associated with tissue sealers were seal failure with bleeding or leakage (Thunderbeat - 18.5\%, Harmonic Ace - 28.4\%) and residual heat (Thunderbeat $-28.4 \%$, Harmonic Ace $-15.7 \%$ ). Both user and device errors were prevalent in the assessment of surgical device failure modes. Despite the presence of user error, the recall reasons that were consistent with the adverse event outcomes and the corresponding number of units recalled indicated that device based failures also share responsibility. The failure modes identified and the phases in which they occurred were consistent with those outlined in scientific literature (Deng, Meng et al. 2002, Champion and Williams 2003, Hamilton, Sims et al. 2003, Marshall, Srivastava et al. 2003, Brown and Woo 2004, Govekar, Robinson et al. 2011, Noble, Smart et al. 2011, Hassouna and Manikandan 2012, Lyons and Law 2013, Mansour, El-Nashar et al. 2014, Overbey, Townsend et al. 2015).

The severity of failure outcomes was assessed in Chapter III. The phases of operation associated with each device type were regrouped into common phases of operation: transition, main function, and results and post-op. The outcome severity of 
failures associated with the surgical devices was significantly different per common phase of operation $(p<0.05)$, indicating an association between the phases of surgical device operation and the severity of outcomes that occurred in each phase. Across both device types, the majority of failure occurred during execution of the devices' main function (51.3\%), which involves securing the tissue (either via stapling or sealing) and transecting tissue (either via knife or thermal/mechanical energy). The average severity of the outcomes that occurred during the main function was 2.80 , which is between moderate and severe on the CTCAEv4.0 grading scale. The least amount of failures occurred during the results and post-op phase of operation (18.8\%); however, the failures that occurred during this phase resulted in the highest average outcome severity of 3.55 , which falls between severe and life-threatening on the CTCAE v4.0 grading scale. The results and post-op phase involved the seal or staple line after activation or firing, respectively, and adverse events that occurred post-operatively.

Various failures modes were uncommon, yet resulted in severe outcomes when they did occur. Therefore, assessing both the frequency of failure modes and the corresponding outcome severity was necessary to provide a more comprehensive assessment of adverse event outcomes. Overall, $72.8 \%$ of failures resulted in an average outcome severity of 3 or higher, which involved a medically significant outcome deemed either severe, life-threatening, or morbid based on the CTCAE v4.0 grading scale.

In Chapter IV, the complexity scores of the surgical devices were determined based on a measure of the requisite steps for structural disassembly, i.e. decomposability. The complexity scores were further utilized in Chapter V, in which the relationship between outcome severity and device design complexity was explored. The Covidien 
Endo GIA had the highest complexity score (478) in addition to the highest number of components (89) and resulted in an average outcome severity of 2.99. The Ethicon Harmonic Ace had the lowest complexity score (170) and resulted in an average outcome severity of 2.65. The Ethicon ETS resulted in a complexity score of 184 and the highest average outcome severity of 3.17. The Thunderbeat resulted in the lowest average outcome severity of 2.63 and a complexity score of 214 . The average outcome severity per device did not increase as the corresponding complexity scores per device increased.

While there was not sufficient evidence to prove a relationship between complexity scores and the average outcome severity of the associated adverse events, a trend in outcome severity existed in the severities per the device types. The endoscopic staplers assessed resulted in higher average outcome severities relative to that of the tissue sealer. Though, the tissue sealer functional model consisted of more functions and resulted in a higher complexity score relative to the endoscopic stapler in order to achieve the main function of securing and separating tissue.

Development of an outcome severity predictive tool based on complexity was explored by performing ordinal regression. Recall from the discussion in Section 5.4, the odds ratios indicated that the outcome severity associated with tissue sealer complexities scores 170 and 214 are likely to be lower than that of both endoscopic stapler complexities of 184 and 478 (odds ratios $<1$ ), a statistically significant effect $(p<0.05$ ). Conversely, the odds ratios indicated that the outcome severity associated with endoscopic stapler complexities 184 and 478 are likely to be higher than that of tissue sealer complexities 170 and 214 (odds ratios $>1$ ), also a statistically significant effect ( $p$ $<0.05)$. 
While a severity predictive tool could not be developed from this particular data set for complexity based on a continuous variable, the ordinal regression analysis provided relative insight for the outcome severities specifically in association with the devices and corresponding complexity scores assessed. Development of a robust predictive tool requires assessment of the severity of adverse event outcomes and design complexity scores of additional minimally invasive surgical devices. Statistical conclusions regarding the overall research goal could not be drawn; however, heuristic methods support the development of the approach to investigating adverse event outcomes from a design complexity perspective.

Evaluating the complexity of surgical devices can provide valuable insight into specific mechanisms of failure and a means to implement countermeasures against undesired outcomes in the surgical setting. The enhancement and modification of current medical devices can be weighed via quantifying the complexity; specifically, the changes in device performance generated by the addition or modification of various features should be weighed in relation to the potential corresponding changes in design complexity. The decomposability approach used herein can aid identification of specific variables that contribute to design complexity, such as connectivity and number of components. Though, analysis of the adverse event reports indicates both user and device-based failures. Further sorting of the adverse events based on device-based failures specifically may be valuable and more applicable to the calculation of complexity based on decomposability. Furthermore, the outcome severity of user-based failures may be more applicable to a score that assesses the elements of device instructions and operation in addition to user experience and knowledge. 
The failure modes and the recalls associated with the surgical devices indicated prevalence of both user and device-related issues. The root cause of failure, whether due to user error or device malfunction, could not be explicitly derived from the adverse event report descriptions in every case. Insufficient descriptions likely trace back to the knowledge gap in user's understanding of surgical device performance and use as demonstrated by the inconsistencies in terminology and narratives associated with adverse event reporting. Though, such findings support an investigation into adverse events from both a clinical and design perspective. All challenges associated with surgical device use and failure must be determined and addressed; the multi-domain approach presented encompasses pertinent techniques to aid this task.

By investigating devices that utilize available and advancing technologies from various manufacturers, this research examined a broad range of operationally diverse classes of surgical devices. As a result, the work is relevant to an array of surgical applications and contributes knowledge to a widespread audience that encompasses users and designers. The approach presented is applicable to the various brands of endoscopic staplers and tissue sealers used in MIS. Also, assessing the adverse event outcome severity and the complexity associated with other classifications of surgical device types using this approach is necessary and appropriate. An essential component of this research involved integrating clinical and design aspects to assist the successful implementation and evaluation of new and modified surgical device technology in the operating room.

It has been estimated that over one million serious injuries to Americans are caused by medical devices each year (Rados 2003). Each year more than 2,000 device- 
related deaths and more than 200,000 reports of device-related injuries and malfunctions are reported to the FDA (Mouzoon and Carome 2012). An investigation of adverse event outcomes that utilizes a severity grading method and a means to quantify and assess complexity is applicable to a broad range of medical devices and corresponding applications. The method described for investigating adverse events is valid for all such products that have the potential for failure and catastrophic outcomes.

Knowledge and experience is crucial for the recognition of the onset of specific failures, which reiterates the importance of filling the knowledge gap encompassing issues related to device use, performance, tissue interaction, potential failure, and preventative action (Deng, Meng et al. 2002, McColl, Karmali et al. 2009, Feldman, Fuchshuber et al. 2012, Feldman, Brunt et al. 2013, Madani, Watanabe et al. 2014, Riggs, Bohm et al. 2016). This study laid a foundation for utilizing severity grades in the assessment of adverse event outcomes retrieved from the FDA MAUDE associated with minimally invasive surgical devices. A novel approach was presented that utilizes a complexity metric to assess design and functional elements of the devices in order to investigate the effect on adverse event outcomes. The new approach initiated by this work enhances risk awareness and prevention techniques via application of the CTCAE v4.0 grading method to adverse event outcomes. The failure mode type, frequency, and outcome severity data presented efficiently outlined and provided more comprehensive content to aid designer and medical professional's education and understanding of the risks associated with various device types that are applicable to a range of procedures.

Development of new methods that integrate clinical and design perspectives is imperative to enhance the safety of a device's final design and improve patient outcomes. 
While the incidence of device failures has been shown to be relatively low compared to overall device usage, it is essential to continuously work towards prevention of adverse event outcomes due to the potential for catastrophic patient consequences (Chan, Bishoff et al. 2000, Deng, Meng et al. 2002). The work from this study serves as a contribution to filling the knowledge gap regarding device use and failure in addition to bridging the gap between users and manufacturers of minimally invasive surgical devices. Thorough and on-going investigation into device complexity and adverse event outcomes as outlined by the approach herein is vital to ensuring patient safety and preventing serious injury and death in the intraoperative procedural setting. 


\section{REFERENCES}

Ahmadi, R., T. A. Roemer and R. H. Wang (2001). "Structuring product development processes." European Journal of Operational Research 130(3): 539-558. DOI: http://dx.doi.org/10.1016/S0377-2217(99)00412-9.

Alkatout, I., T. Schollmeyer, N. A. Hawaldar, N. Sharma and L. Mettler (2012). "Principles and Safety Measures of Electrosurgery in Laparoscopy." JSLS : Journal of the Society of Laparoendoscopic Surgeons 16(1): 130-139. DOI: 10.4293/108680812X13291597716348.

Ameri, F., J. D. Summers, G. M. Mocko and M. Porter (2008). "Engineering design complexity: an investigation of methods and measures." Research in Engineering Design 19(2): 161-179. DOI: 10.1007/s00163-008-0053-2.

Angrisani, L., M. Lorenzo, V. Borrelli, M. Ciannella, U. A. Bassi and P. Scarano (2004). "The use of bovine pericardial strips on linear stapler to reduce extraluminal bleeding during laparoscopic gastric bypass: prospective randomized clinical trial." Obes Surg 14(9): 1198-1202. DOI: 10.1381/0960892042387075.

Austin, P. C. and E. W. Steyerberg (2015). "The number of subjects per variable required in linear regression analyses." Journal of Clinical Epidemiology 68(6): 627-636. DOI: http://dx.doi.org/10.1016/j.jclinepi.2014.12.014.

Aytan, H., H. Nazik, R. Narin, M. Api and E. C. Tok (2014). "Comparison of the Use of LigaSure, HALO PKS Cutting Forceps, and ENSEAL Tissue Sealer in Total Laparoscopic Hysterectomy: A Randomized Trial." Journal of Minimally Invasive Gynecology 21(4): 650-655. DOI: http://dx.doi.org/10.1016/j.jmig.2014.01.010.

Baker, R. S., J. Foote, P. Kemmeter, R. Brady, T. Vroegop and M. Serveld (2004). "The Science of Stapling and Leaks." Obesity Surgery 14(10): 1290-1298. DOI: 10.1381/0960892042583888.

Baker, S. P., B. O'Neill, W. Haddon, Jr. and W. B. Long (1974). "The injury severity score: a method for describing patients with multiple injuries and evaluating emergency care." J Trauma 14(3): 187-196. 
Basch, E., A. Iasonos, T. McDonough, A. Barz, A. Culkin, M. G. Kris, H. I. Scher and D. Schrag (2006). "Patient versus clinician symptom reporting using the National Cancer Institute Common Terminology Criteria for Adverse Events: results of a questionnaire-based study." The Lancet Oncology 7(11): 903-909. DOI: http://dx.doi.org/10.1016/S1470-2045(06)70910-X.

Belli, G., C. Fantini, A. D'Agostino, A. Belli, L. Cioffi and N. Russolillo (2006). "Laparoscopic left lateral hepatic lobectomy: a safer and faster technique." Journal of Hepato-Biliary-Pancreatic Surgery 13(2): 149-154. DOI: 10.1007/s00534-005-1023$\mathrm{y}$.

Biere, S. S. A. Y., M. I. van Berge Henegouwen, K. W. Maas, L. Bonavina, C. Rosman, J. R. Garcia, S. S. Gisbertz, J. H. G. Klinkenbijl, M. W. Hollmann, E. S. M. de Lange, H. J. Bonjer, D. L. van der Peet and M. A. Cuesta (2012). "Minimally invasive versus open oesophagectomy for patients with oesophageal cancer: a multicentre, openlabel, randomised controlled trial." The Lancet 379(9829): 1887-1892. DOI: 10.1016/S0140-6736(12)60516-9.

Blackenfelt, M. (2001). Managing complexity by product modularisation Doctoral, Maskinkonstruktion KTH.

Bozarth, C. C., D. P. Warsing, B. B. Flynn and E. J. Flynn (2009). "The impact of supply chain complexity on manufacturing plant performance." Journal of Operations Management 27(1): 78-93. DOI: http://dx.doi.org/10.1016/j.jom.2008.07.003.

Braha, D. and O. Maimon (1998). The Measurement of a Design Structural and Functional Complexity. IEEE Transactions on Systems, Man, and Cybernetics - Part A: Systems and Humans.

Bridges, M. and D. L. Diamond (1999). "The financial impact of teaching surgical residents in the operating room." The American Journal of Surgery 177(1): 28-32. DOI: http://dx.doi.org/10.1016/S0002-9610(98)00289-X.

Broughton, D., A. L. Welling, E. H. Monroe, K. Pirozzi, J. B. Schulte and J. W. Clymer (2013). "Tissue effects in vessel sealing and transection from an ultrasonic device with more intelligent control of energy delivery." Med Devices (Auckl) 6: 151-154. DOI: $10.2147 /$ mder.s51663.

Brown, S. L. and E. K. Woo (2004). "Surgical stapler-associated fatalities and adverse events reported to the Food and Drug Administration." Journal of the American College of Surgeons 199(3): 374-381. DOI: http://dx.doi.org/10.1016/j.jamcollsurg.2004.05.264.

Bruce, J., E. M. Russell, J. Mollison and Z. H. Krukowski (2001). "The measurement and monitoring of surgical adverse events." Health Technol Assess 5(22): 1-194.

Burditt, G. M. (1995). "The history of food law." Food Drug Law J 50 Spec: 197-201. 
Carrascosa, M., S. D. Eppinger and D. E. Whitney (1998). Using the design structure matrix to estimate product development time. Proceedings of the ASME design engineering technical conferences (design automation conference).

CDC. (2016, Aug 22, 2016). "International Classification of Diseases, Tenth Revision, Clinical Modification (ICD-10-CM)." National Center for Health Statistics Retrieved Sep 12, 2016, from http://www.cdc.gov/nchs/icd/icd10cm.htm.

CDHR. (2017, 09/01/2016). "CDRH Management Directory by Organization." Office of Device Evaluation, from http://www.fda.gov/AboutFDA/CentersOffices/OfficeofMedicalProductsandTobacco/ CDRH/CDRHOffices/ucm127854.htm.

Challoner, D. R. (2011). "Medical Devices and the Public's Health: The FDA 510(k) Clearance Process at 35 Years " Institute of Medicine of the National Academies. 6.

Champion, J. K. and M. D. Williams (2003). "Prospective randomized comparison of linear staplers during laparoscopic Roux-en-Y gastric bypass." Obes Surg 13(6): 855859; discussion 860. DOI: 10.1381/096089203322618641.

Chan, D., J. T. Bishoff, L. Ratner, L. R. Kavoussi and T. W. Jarrett (2000). "Endo vascular Gastrointestinal Stapler Device Malfunction During Laparoscopic Nephrectomy: Early Recognition and Management." The Journal of Urology 164(2): 319-321. DOI: http://dx.doi.org/10.1016/S0022-5347(05)67349-1.

Chekan, E. and R. L. Whelan (2014). "Surgical stapling device-tissue interactions: what surgeons need to know to improve patient outcomes." Medical Devices (Auckland, N.Z.) 7: 305-318. DOI: 10.2147/MDER.S67338.

Chekan, E., R. L. Whelan and A. H. Feng (2013). "Device-tissue interactions: a collaborative communications system." Annals of Surgical Innovation and Research 7: 10-10. DOI: 10.1186/1750-1164-7-10.

Closs, D. J., M. A. Jacobs, M. Swink and G. S. Webb (2008). "Toward a theory of competencies for the management of product complexity: six case studies." Journal of Operations Management 26(5): 590-610.

Closs, D. J., G. N. Nyaga and M. D. Voss (2010). "The differential impact of product complexity, inventory level, and configuration capacity on unit and order fill rate performance." Journal of Operations Management 28(1): 47-57. DOI: http://dx.doi.org/10.1016/j.jom.2009.04.003.

Copes, W. S., H. R. Champion, W. J. Sacco, M. M. Lawnick, D. S. Gann, T. Gennarelli, E. MacKenzie and S. Schwaitzberg (1990). "Progress in characterizing anatomic injury." J Trauma 30(10): 1200-1207. 
Covidien. (2017). "Medtronic, Covidien Products." Endo GIATM Universal Staplers and Reloads Retrieved June, 2017, from http://products.covidien.com/pages.aspx?page=ModelDetail\&cat=Devices $\&$ cat $2=\mathrm{Mo}$ del\&id=7396\&_ga $=2.178682916 .1494538157 .1500389403-$ 1007491567.1497025209.

Cuschieri, A. (2006). "Nature of human error: implications for surgical practice." Ann Surg 244(5): 642-648. DOI: 10.1097/01.sla.0000243601.36582.18.

Dankelman, J. and C. A. Grimbergen (2005). "Systems approach to reduce errors in surgery." Surg Endosc 19(8): 1017-1021. DOI: 10.1007/s00464-005-8109-0.

Dekutoski, M. B., D. C. Norvell, J. R. Dettori, M. G. Fehlings and J. R. Chapman (2010). "Surgeon perceptions and reported complications in spine surgery." Spine (Phila Pa 1976) 35(9 Suppl): S9-s21. DOI: 10.1097/BRS.0b013e3181d830de.

Deng, D. Y., M. V. Meng, H. T. Nguyen, G. C. Bellman and M. L. Stoller (2002). "Laparoscopic linear cutting stapler failure." Urology 60(3): 415-419. DOI: http://dx.doi.org/10.1016/S0090-4295(02)01778-8.

Dindo, D., N. Demartines and P. A. Clavien (2004). "Classification of surgical complications: a new proposal with evaluation in a cohort of 6336 patients and results of a survey." Ann Surg 240(2): 205-213.

ElMaraghy, W., H. ElMaraghy, T. Tomiyama and L. Monostori (2012). "Complexity in engineering design and manufacturing." CIRP Annals - Manufacturing Technology 61(2): 793-814. DOI: http://dx.doi.org/10.1016/j.cirp.2012.05.001.

English, K. W. and C. L. Bloebaum (2008). "Visual Dependency Structure Matrix for Multidisciplinary Design Optimization Tradeoff Studies." Journal of Aerospace Computing, Information, and Communication 5(9): 274-297. DOI: 10.2514/1.32038.

Entezari, K., P. Hoffmann, M. Goris, A. Peltier and R. Van Velthoven (2007). "A review of currently available vessel sealing systems." Minimally Invasive Therapy \& Allied Technologies 16(1): 52-57. DOI: 10.1080/13645700601181414.

Eppinger, S. D. and T. R. Browning (2012). Design structure matrix methods and applications, MIT press.

Ethicon. (2017). "Ethicon, Part of the Johnson \& Johnson Family of Companies." Surgical Stapling, ENDOPATH® ETS Articulating Linear Cutters Retrieved June, 2017, from http://www.ethicon.com/healthcareprofessionals/products/staplers/endocutters/endopath-ets-articulating-linear.

Ethicon. (2017). "Ethicon, Part of the Johnson \& Johnson Family of Companies." HARMONIC ACE $®$ + Shears with Adaptive Tissue Technology Retrieved June, 2017, from http://www.ethicon.com/healthcare-professionals/products/advancedenergy/harmonic/harmonic-ace-plus. 
Farid, A. M. and D. C. McFarlane (2006). An approach to the application of the design structure matrix for assessing reconfigurability of distributed manufacturing systems. Distributed Intelligent Systems: Collective Intelligence and Its Applications. DOI: http://doi.ieeecomputersociety.org/10.1109/DIS.2006.10.

Farnworth, L. R., D. E. Lemay, T. Wooldridge, J. D. Mabrey, M. J. Blaschak, T. A. DeCoster, D. C. Wascher and R. C. Schenck (2001). "A Comparison of Operative Times in Arthroscopic ACL Reconstruction Between Orthopaedic Faculty and Residents: The Financial Impact of Orthopaedic Surgical Training in the Operating Room." The Iowa Orthopaedic Journal 21: 31-35.

FDA. (2009). "Safety." Background and Definitions Retrieved March 22, 2017, from https://www.fda.gov/Safety/Recalls/ucm165546.htm.

FDA. (2016). "What is a Serious Adverse Event?" Safety Retrieved June 13, 2017, from https://www.fda.gov/safety/medwatch/howtoreport/ucm053087.htm.

FDA. (2017). "MAUDE - Manufacturer and User Facility Device Experience " Retrieved June 13, 2017, from https://www.accessdata.fda.gov/scripts/cdrh/cfdocs/cfmaude/search.cfm.

FDA. (2017, 07/16/2015). "Medical Device Reporting (MDR)." from http://www.fda.gov/MedicalDevices/Safety/ReportaProblem/ucm2005291.htm.

FDA. (2017, 06/02/2014). "What is a Medical Device Recall?" Retrieved September 2, 2016, from http://www.fda.gov/MedicalDevices/Safety/ListofRecalls/ucm329946.htm.

Feldman, L. S., L. M. Brunt, P. Fuchshuber, D. B. Jones, S. B. Jones, J. Mischna, M. G. Munro, M. A. Rozner and S. D. Schwaitzberg (2013). "Rationale for the Fundamental Use of Surgical Energy ${ }^{\mathrm{TM}}$ (FUSE) curriculum assessment: focus on safety." Surgical Endoscopy 27(11): 4054-4059. DOI: 10.1007/s00464-013-3059-4.

Feldman, L. S., P. Fuchshuber, D. B. Jones, J. Mischna and S. D. Schwaitzberg (2012). "Surgeons don't know what they don't know about the safe use of energy in surgery." Surgical Endoscopy 26(10): 2735-2739. DOI: 10.1007/s00464-012-2263-y.

Flin, R., P. O'Connor and M. Crichton (2008). Safety at the Sharp End: A Guide to NonTechnical Skills. Boca Raton, FL, CRC Press.

Fuller, H. J. A., D. E. Paull and L. C. Williams (2014). "Surgical Stapler Adverse Events in the Veterans Health Administration: Root Causes and Lessons Learned." International Symposium on Human Factors and Ergonomics in Health Care: Advancing the Cause: $153-156$. DOI: $10.1177 / 2327857914031025$.

Fuller, J., B. S. Ashar and J. Carey-Corrado (2005). "Trocar-associated injuries and fatalities: An analysis of 1399 reports to the FDA." Journal of Minimally Invasive Gynecology 12(4): 302-307. DOI: http://dx.doi.org/10.1016/j.jmig.2005.05.008. 
Gallagher, K., B. Dhinsa and J. Miles (2011). "Electrosurgery." Surgery - Oxford International Edition 29(2): 70-72. DOI: 10.1016/j.mpsur.2010.11.009.

GAO. (2009). "FDA Should Take Steps to Ensure That High-Risk Device Types Are Approved through the Most Stringent Premarket Review Process." GAO-09-190 Retrieved September 2, 2016, from http://www.gao.gov/assets/290/284882.pdf.

Govekar, H. R., T. N. Robinson, G. V. Stiegmann and F. T. McGreevy (2011). "Residual heat of laparoscopic energy devices: how long must the surgeon wait to touch additional tissue?" Surgical Endoscopy 25(11): 3499. DOI: 10.1007/s00464-0111742-x.

Green, S. B. (1991). "How Many Subjects Does It Take To Do A Regression Analysis." Multivariate Behavioral Research 26(3): 499-510. DOI: 10.1207/s15327906mbr2603_7.

Guenov, M. D. and S. G. Barker (2005). "Application of axiomatic design and design structure matrix to the decomposition of engineering systems." Systems Engineering 8(1): 29-40. DOI: $10.1002 /$ sys.20015.

Gupta, S. P. and A. Pidgeon (2016). "An analytical approach to identifying potential userelated issues concerning a medical device under development." Journal of Medical Engineering \& Technology 40(3): 61-71. DOI: 10.3109/03091902.2015.1132785.

Hagedorn, T. J., I. R. Grosse and S. Krishnamurty (2015). "A concept ideation framework for medical device design." J Biomed Inform 55: 218-230. DOI: 10.1016/j.jbi.2015.04.010.

Hall, S. G., R. J. Tanguay, J. D. Messerly, G. C. Robertson, A. M. Zwolinski and F. E. S. IV (2011). Surgical Stapling Apparatus with Interlockable Firing System. United States, Ethicon Endo-Surgery, Inc. 7,866,527 B2.

Hamilton, E. C., T. L. Sims, T. T. Hamilton, M. A. Mullican, D. B. Jones and D. A. Provost (2003). "Clinical predictors of leak after laparoscopic Roux-en-Y gastric bypass for morbid obesity." Surg Endosc 17(5): 679-684. DOI: 10.1007/s00464-0028819-5.

Handy, J. R., J. W. Asaph, E. C. Douville, G. Y. Ott, G. L. Grunkemeier and Y. Wu (2010). "Does video-assisted thoracoscopic lobectomy for lung cancer provide improved functional outcomes compared with open lobectomy?" European Journal of Cardio-Thoracic Surgery 37(2): 451-455. DOI: 10.1016/j.ejcts.2009.07.037.

Harrell, G. J. and D. R. Kopps (1998). "Minimizing Patient Risk During Laparoscopic Electrosurgery." AORN Journal 67(6): 1199-1202. DOI: 10.1016/S00012092(06)62606-9. 
Hassouna, H. A. and R. Manikandan (2012). "Hemostasis in laparoscopic renal surgery." Indian Journal of Urology : IJU : Journal of the Urological Society of India 28(1): 38. DOI: 10.4103/0970-1591.94939.

Hay, D. J. (2005). "Basic skills: Electrosurgery." Surgery (Oxford) 23(2): 73-75. DOI: http://dx.doi.org/10.1383/surg.23.2.73.60349.

Hirtz, J., R. Stone, D. McAdams, S. Szykman and K. Wood (2002). "A Functional Basis for Engineering Design: Reconciling and Evolving Previous Efforts." Research in Engineering Design 13(2): 65-82.

Hmtema, T. (1998). Articulation Transmission Mechanism for Surgical Instruments. United States, Ethicon Endo-Surgery, Inc. 5,713,505.

Hölttä, K. M. M. and K. N. Otto (2005). "Incorporating design effort complexity measures in product architectural design and assessment." Design Studies 26(5): 463485. DOI: http://dx.doi.org/10.1016/j.destud.2004.10.001.

Hommes, Q. D. V. E. and P. W. Berry (2003). "4.4.3 Managing System Interface Requirements Reconciliation Using Design Structure Matrix Method." INCOSE International Symposium 13(1): 607-618. DOI: 10.1002/j.2334-5837.2003.tb02645.x.

Hruby, G. W., F. C. Marruffo, E. Durak, S. M. Collins, P. Pierorazio, P. A. Humphrey, M. M. Mansukhani and J. Landman (2007). "Evaluation of surgical energy devices for vessel sealing and peripheral energy spread in a porcine model." J Urol 178(6): 2689-2693. DOI: 10.1016/j.juro.2007.07.121.

Hu, J. C., X. Gu, S. R. Lipsitz and et al. (2009). "Comparative effectiveness of minimally invasive vs open radical prostatectomy." JAMA 302(14): 1557-1564. DOI: 10.1001/jama.2009.1451.

Huitema, T. W., A. K. Nalagatla and D. R. Schulze (1998). Articulation Assembly for Surgical Instruments. United States, Ethicon Endo-Surgery, Inc. 5,704,534.

Hundal, M. (1990). "A Systematic Method for Developing Function Structures, Solutions and Concept Variants." Mechanism and Machine Theory 25(3): 243-256. DOI: http://dx.doi.org/10.1016/0094-114X(90)90027-H.

Irwin, M. G. and S. S. C. Wong (2012). "Anaesthesia and minimally invasive surgery." Anaesthesia \& Intensive Care Medicine 13(2): 48-51. DOI: http://dx.doi.org/10.1016/j.mpaic.2011.11.004.

IV, F. E. S., M. E. Setser and W. B. W. III (2005). Surgical Stapling Instrument Incorporating an E-Beam Firing Mechanism. United States, Ethicon Endo-Surgery, Inc. 6,978,921 B2.

Jacobs, M. A. (2013). "Complexity: Toward an empirical measure." Technovation 33(45): 111-118. DOI: http://dx.doi.org/10.1016/j.technovation.2013.01.001. 
Janetschek, G., F. Bagheri, A. Abdelmaksoud, C. S. Biyani, K. Leeb and S. Jeschke (2003). "Ligation of the Renal Vein During Laparoscopic Nephrectomy: An Effective And Reliable Method to Replace Vascular Staplers." The Journal of Urology 170(4, Part 1): 1295-1297. DOI: http://dx.doi.org/10.1097/01.ju.0000081303.79650.91.

JnJ. (2016). "Johnson \& Johnson 2015 Annual Report." Major Medical Devices Franchise Sales. New Brunswick, New Jersey. Johnson \& Johnson. 16.

Johnson, K. D., G. M. Couture, J. Unger, R. Sharp, D. M. Garrison, S. T. Dycus, D. Hixson and C. Weinberg (2007). Vessel Sealing Instrument with Electrical Cutting Mechanism. United States, Sherwood Services AG. US7270664B2: 34.

Jones, D. B., L. M. Brunt, L. S. Feldman, D. J. Mikami, T. N. Robinson and S. B. Jones (2015). "Safe energy use in the operating room." Curr Probl Surg 52(11): 447-468. DOI: $10.1067 /$ j.cpsurg.2015.08.004.

Koller, R. (1985). Konstruktionslehre für den Maschinenbau (Mechanical Engineering Design). Berlin: Springer-Verlag.

Kurfman, M., R. Stone, M. Van Wie, K. Wood and K. Otto (2000). Theoretical Underpinnings of Functional Modeling: Preliminary Experimental Studies. Proceedings of DETC2000. Balitmore, MD. DETC2000/DTM-14563.

Kwazneski, D., C. Six and K. Stahlfeld (2012). "The unacknowledged incidence of laparoscopic stapler malfunction." Surgical Endoscopy 27(1): 86-89. DOI: 10.1007/s00464-012-2417-y.

Lambe, A. B. and J. R. Martins (2012). "Extensions to the design structure matrix for the description of multidisciplinary design, analysis, and optimization processes." Structural and Multidisciplinary Optimization 46(2): 273-284.

Lamberton, G. R., R. S. Hsi, D. H. Jin, T. U. Lindler, F. C. Jellison and D. D. Baldwin (2008). "Prospective comparison of four laparoscopic vessel ligation devices." J Endourol 22(10): 2307-2312. DOI: 10.1089/end.2008.9715.

Lavernia, C. J., R. J. Sierra and R. A. Hernandez (2000). "The Cost of Teaching Total Knee Arthroplasty Surgery to Orthopaedic Surgery Residents." Clinical Orthopaedics and Related Research 380: 99-107.

Liberman, M., M. Khereba, E. Goudie, J. Kazakov, V. Thiffault, E. Lafontaine and P. Ferraro (2014). "Pilot study of pulmonary arterial branch sealing using energy devices in an ex vivo model." The Journal of Thoracic and Cardiovascular Surgery 148(6): 3219-3223. DOI: http://dx.doi.org/10.1016/j.jtcvs.2014.05.089.

Little, A., K. Wood and D. McAdams (1997). Functional Analysis: A Fundamental Empirical Study for Reverse Engineering, Benchmarking and Redesign. Proceedings of the 1997 Design Engineering Technical Conferences. Sacramento, CA. 97DETC/DTM-3879. 
Liu, Y.-J., G.-P. Zhu and X.-Y. Guan (2012). "Comparison of the NCI-CTCAE version 4.0 and version 3.0 in assessing chemoradiation-induced oral mucositis for locally advanced nasopharyngeal carcinoma." Oral Oncology 48(6): 554-559. DOI: http://dx.doi.org/10.1016/j.oraloncology.2012.01.004.

Lough, K. G., R. B. Stone and I. Tumer (2008). "Implementation Procedures for the Risk in Early Design (RED) Method." Journal of Industrial and Systems Engineering 2(2): 126-143.

Lyons, S. D. and K. S. K. Law (2013). "Laparoscopic Vessel Sealing Technologies." Journal of Minimally Invasive Gynecology 20(3): 301-307. DOI: http://dx.doi.org/10.1016/j.jmig.2013.02.012.

Madani, A., Y. Watanabe, N. Townsend, P. H. Pucher, T. N. Robinson, P. E. Egerszegi, J. Olasky, S. L. Bachman, C. W. Park, N. Amin, D. T. Tang, E. Haase, D. Bardana, D. B. Jones, M. Vassiliou, G. M. Fried and L. S. Feldman (2016). "Structured simulation improves learning of the Fundamental Use of Surgical Energy ${ }^{\mathrm{TM}}$ curriculum: a multicenter randomized controlled trial." Surgical Endoscopy 30(2): 684-691. DOI: 10.1007/s00464-015-4260-4.

Madani, A., Y. Watanabe, M. C. Vassiliou, P. Fuchshuber, D. B. Jones, S. D. Schwaitzberg, G. M. Fried and L. S. Feldman (2014). "Impact of a hands-on component on learning in the Fundamental Use of Surgical Energy ${ }^{\mathrm{TM}}$ (FUSE) curriculum: a randomized-controlled trial in surgical trainees." Surgical Endoscopy 28(10): 2772-2782. DOI: 10.1007/s00464-014-3544-4.

Madani, A., Y. Watanabe, M. C. Vassiliou, P. Fuchshuber, D. B. Jones, S. D. Schwaitzberg, G. M. Fried and L. S. Feldman (2016). "Long-term knowledge retention following simulation-based training for electrosurgical safety: 1-year follow-up of a randomized controlled trial." Surgical Endoscopy 30(3): 1156-1163. DOI: $10.1007 / \mathrm{s} 00464-015-4320-9$.

Maier, M. W. and E. Rechtin (2000). The art of systems architecture, Boca Raton: CRC Press.

Maisel, W. H. (2004). "Medical device regulation: an introduction for the practicing physician." Ann Intern Med 140(4): 296-302.

Maisel, W. H. (2005). "Safety issues involving medical devices: Implications of recent implantable cardioverter-defibrillator malfunctions." JAMA 294(8): 955-958. DOI: 10.1001/jama.294.8.955.

Makins, B. J. and D. W. Miller (2000). "1.2.3 Web-based Aerospace System Evaluation Software: The Development and Assessment of Conceptual Space Missions." INCOSE International Symposium 10(1): 157-164. DOI: 10.1002/j.23345837.2000.tb00371.x. 
Mansour, A. M., O. El-Nashar, A. R. El-Nahas, B. Ali-El-Dein, Y. Osman, A. A. Shokeir and I. Eraky (2014). "Endovascular-GIA Stapler Device Malfunction During Laparoscopic Nephrectomy: A Comprehensive Analysis of the FDA-Manufacturer and User Facility Device Experience (MAUDE) Database." The Journal of Urology 191(4): e356. DOI: 10.1016/j.juro.2014.02.996.

Marczyk, S. (2006). Surgical Stapling Apparatus with Locking Mechanism. United States, Tyco Healthcare Group LP. 7,097,089 B2.

Marshall, J., A. Srivastava, S. K. Gupta, T. R. Rossi and J. R. DeBord (2003). "Roux-eny gastric bypass leak complications." Archives of Surgery 138(5): 520-524. DOI: 10.1001/archsurg.138.5.520.

Massarweh, N. N., N. Cosgriff and D. P. Slakey (2006). "Electrosurgery: History, Principles, and Current and Future Uses." Journal of the American College of Surgeons 202(3): 520-530. DOI: http://dx.doi.org/10.1016/j.jamcollsurg.2005.11.017.

McColl, R. J., S. Karmali, A. Reso, E. Paolucci and V. Sherman (2009). "The Effect of a Focused Instructional Session on Knowledge of Surgical Staplers in General Surgery Residents." Journal of Surgical Education 66(5): 288-291. DOI: http://dx.doi.org/10.1016/j.jsurg.2009.08.003.

McCrory, B., C. A. LaGrange and M. S. Hallbeck (2014). "Quality and Safety of Minimally Invasive Surgery: Past, Present, and Future." Biomedical Engineering and Computational Biology 6: 1-11. DOI: 10.4137/BECB.S10967.

Mery, C. M., B. M. Shafi, G. Binyamin, J. M. Morton and M. Gertner (2008). "Profiling surgical staplers: effect of staple height, buttress, and overlap on staple line failure." Surgery for Obesity and Related Diseases 4(3): 416-422. DOI: http://dx.doi.org/10.1016/j.soard.2007.11.008.

Miles, L. (1972). Techniques of Value Analysis and Engineering. New York, McGrawHill.

Milsom, J., K. Trencheva, S. Monette, R. Pavoor, P. Shukla, J. Ma and T. Sonoda (2012). "Evaluation of the safety, efficacy, and versatility of a new surgical energy device (THUNDERBEAT) in comparison with Harmonic ACE, LigaSure V, and EnSeal devices in a porcine model." J Laparoendosc Adv Surg Tech A 22(4): 378-386. DOI: 10.1089/lap.2011.0420.

Mitchell, R. J., A. Williamson and B. Molesworth (2015). "Use of a human factors classification framework to identify causal factors for medication and medical devicerelated adverse clinical incidents." Safety Science 79: 163-174. DOI: http://dx.doi.org/10.1016/j.ssci.2015.06.002.

Mohinder, S. R., A. Gill and J. Summers (2016). Using Graph Complexity Connectivity Method to Predict Information from Design Representations: A Comparative Study. Design Computing and Cognition DCC'16. Chicago, IL, Springer. 
Monsein, L. H. (1997). "Primer on medical device regulation. Part I. History and background." Radiology 205(1): 1-9. DOI: 10.1148/radiology.205.1.9314952.

Mountain, P. J., M. R. Bohm and M. K. Riggs (2016). Evaluation of the Techniques to Describe Device Complexity in Pre and Post Design Stages. ASME 2016 International Mechanical Engineering Congress \& Exposition. Phoenix, Arizona.

Mouzoon, N. and M. Carome (2012) "Substantially Unsafe." Medical Devices Pose Great Threat to Patients; Safeguards Must be Strengthened, Not Weakened.

Nagel, R. L., M. R. Bohm, J. Cole and P. Shepard (2012). An Algorithmic Approach to Teaching Functionality. ASME 2012 International Design Engineering Technical Conferences and Computers and Information in Engineering Conference, American Society of Mechanical Engineers.

Nagel, R. L., M. R. Bohm, J. S. Linsey and M. K. Riggs (2015). "Improving Students' Functional Modeling Skills: A Modeling Approach and a Scoring Rubric." Journal of Mechanical Design 137(5): 051102. DOI: doi:10.1115/1.4029585.

Nduka, C. C., P. A. Super, J. R. Monson and A. W. Darzi (1994). "Cause and prevention of electrosurgical injuries in laparoscopy." J Am Coll Surg 179(2): 161-170.

NIH (2003) "Common Terminology Criteria for Adverse Events v3.0 (CTCAE)."

NIH (2009) "Common Terminology Criteria for Adverse Events v4.0 (CTCAE)."

Noble, E. J., N. J. Smart, C. Challand, K. Sleigh, A. Oriolowo and K. B. Hosie (2011). "Experimental comparison of mesenteric vessel sealing and thermal damage between one bipolar and two ultrasonic shears devices." Br J Surg 98(6): 797-800. DOI: $10.1002 /$ bjs. 7433 .

Novack, L., A. Jotkowitz, B. Knyazer and V. Novack (2006). "Evidence-based medicine: assessment of knowledge of basic epidemiological and research methods among medical doctors." Postgraduate Medical Journal 82(974): 817-822. DOI: 10.1136/pgmj.2006.049262.

NSW (2014) "Policy Directive: Incidient Management Policy."

Obonna, G. and R. Mishra (2014). "Differences between Thunderbeat, Ligasure and Harmonic Scalpel Energy System in Minimally Invasive Surgery." World Journal of Laparoscopic Surgery 7(1): 41-44. DOI: 10.5005/jp-journals-10033-1215.

Olympus. (2012). "Olympus." News, Olympus to Launch THUNDERBEAT Retrieved March, 2017, from https://www.olympusglobal.com/en/news/2012a/nr120321thunderbeate.html.

Olympus. (2012). "Thunderbeat Tissue Management System: A Reference Guide for Health Care Professionals." Hamburg, Germany. O. E. S. C. KG. 
Olympus. (2017). "Olympus." Advanced Energy, Thunderbeat Retrieved June, 2017, from http://medical.olympusamerica.com/products/thunderbeat-0.

Otto, K. (2001). A Process for Modularizing Product Families. ICED 01: International Conference on Engineering Design, Glasgow; August 21-23, Professional Engineering Publishing Limited.

Otto, K. and K. Wood (2001). "Product design: techniques in reverse engineering and new product design." Prentice-Hall.

Overbey, D. M., N. T. Townsend, B. C. Chapman, D. T. Bennett, L. S. Foley, A. S. Rau, J. A. Yi, E. L. Jones, G. V. Stiegmann and T. N. Robinson (2015). "Surgical EnergyBased Device Injuries and Fatalities Reported to the Food and Drug Administration." Journal of the American College of Surgeons 221(1): 197-205.e191. DOI: http://dx.doi.org/10.1016/j.jamcollsurg.2015.03.031.

Pahl, G. and W. Beitz (2013). Engineering design: a systematic approach, Springer Science \& Business Media.

Pahl, G., W. Beitz, H. Schulz and U. Jarecki (2007). Engineering Design: A Systematic Approach. London, Springer-Verlag.

Parker, W. H. (2010). "Understanding Errors During Laparoscopic Surgery." Obstetrics and Gynecology Clinics of North America 37(3): 437-449. DOI: http://dx.doi.org/10.1016/j.ogc.2010.06.001.

Person, B., D. A. Vivas, D. Ruiz, M. Talcott, J. E. Coad and S. D. Wexner (2008). "Comparison of four energy-based vascular sealing and cutting instruments: A porcine model." Surgical Endoscopy 22(2): 534-538. DOI: 10.1007/s00464-0079619-8.

Porembka, M. R., B. L. Hall, M. Hirbe and S. M. Strasberg (2010). "Quantitative weighting of postoperative complications based on the accordion severity grading system: demonstration of potential impact using the american college of surgeons national surgical quality improvement program." J Am Coll Surg 210(3): 286-298. DOI: 10.1016/j.jamcollsurg.2009.12.004.

Rados, C. (2003). FDA Works to Reduce Preventable Medical Device Injuries. FDA Consumer Magazine.

Riggs, M. K., M. R. Bohm and P. J. Mountain (2016). Examining Relationships Between Device Complexity and Failure Modes of Minimally Invasive Surgical Staplers. ASME 2016 International Mechanical Engineering Congress \& Exposition Phoenix, Arizona. 3: Biomedical and Biotechnology Engineering: V003T004A014.

Robert, M., G. Poncet, J. Boulez, F. Mion and P. Espalieu (2011). "Laparoscopic Gastric Bypass for Failure of Adjustable Gastric Banding: A Review of 85 Cases." Obesity Surgery 21(10): 1513-1519. DOI: 10.1007/s11695-011-0391-4. 
Rodenacker, W. (1971). Methodisches Konstruieren (Methodical Design). Berlin, Heidelberg, New York, Springer.

Roth, K. H. (1981). "Foundation of Methodical Procedures in Design." Design Studies 2(2): 107-115. DOI: http://dx.doi.org/10.1016/0142-694X(81)90007-7.

Samore, M. H., R. Evans, A. Lassen and et al. (2004). "Surveillance of medical devicerelated hazards and adverse events in hospitalized patients." JAMA 291(3): 325-334. DOI: 10.1001/jama.291.3.325.

Sankaranarayanan, G., R. R. Resapu, D. B. Jones, S. Schwaitzberg and S. De (2013). "Common uses and cited complications of energy in surgery." Surgical Endoscopy 27(9): 3056-3072. DOI: 10.1007/s00464-013-2823-9.

Scirica, P. (2010). Surgical Stapling Instrument with Replaceable Loading Unit. United States, Tyco Healthcare Group LP. 2010/0237130 A1.

Seehofer, D., M. Mogl, S. Boas-Knoop, J. Unger, A. Schirmeier, S. Chopra and D. Eurich (2012). "Safety and efficacy of new integrated bipolar and ultrasonic scissors compared to conventional laparoscopic 5-mm sealing and cutting instruments." Surgical Endoscopy 26(9): 2541-2549. DOI: 10.1007/s00464-012-2229-0.

Shikora, S. A., J. J. Kim and M. E. Tarnoff (2003). "Reinforcing Gastric Staple-Lines with Bovine Pericardial Strips May Decrease the Likelihood of Gastric Leak after Laparoscopic Roux-en-Y Gastric Bypass." Obesity Surgery 13(1): 37-44. DOI: $10.1381 / 096089203321136566$.

Soltz, M. and J. Broom (2012). Staple with Multiple Cross Sectional Shapes. United States, Tyco Healthcare Group LP. 2012/0241504 A1.

Stock, M., R. Stone and I. Y. Tumer (2003). Going Back in Time to Improve Design: The Function-Failure Design Method. ASME Design Engineering Technical Conference, Design Theory and Methodology Conference, Chicago, IL.

Stone, R., I. Tumer and M. Van Wie (2004). "The Function Failure Design Method." Journal of Mechanical Design 127(3): 397-407.

Stone, R. and K. Wood (2000). "Development of a Functional Basis for Design." Journal of Mechanical Design 122(4): 359-370. DOI: http://dx.doi.org/10.1115/1.1289637.

Stone, R. B., K. L. Wood and R. H. Crawford (2000). "A heuristic method for identifying modules for product architectures." Design Studies 21(1): 5-31. DOI: http://dx.doi.org/10.1016/S0142-694X(99)00003-4.

Strasberg, S. M., D. C. Linehan and W. G. Hawkins (2009). "The accordion severity grading system of surgical complications." Ann Surg 250(2): 177-186. DOI: 10.1097/SLA.0b013e3181afde41. 
Suh, N. (2001). Axiomatic Design: Advances and Applications. New York, Oxford University Press.

Sullivan, K. J., W. G. Griswold, Y. Cai and B. Hallen (2001). The structure and value of modularity in software design. ACM SIGSOFT Software Engineering Notes, ACM.

Summers, J. D. and F. Ameri (2008). An algorithm for assessing design complexity through a connectivity view. Tools and Methods for Competitive Engineering. I. Horváth and Z. Rusák. Izmir, Turkey.

Summers, J. D. and J. J. Shah (2010). "Mechanical Engineering Design Complexity Metrics: Size, Coupling, and Solvability." Journal of Mechanical Design, Transactions of the ASME 132(2): 210041-2100411. DOI: $10.1115 / 1.4000759$.

Swanson, S. J., B. F. Meyers, C. L. Gunnarsson, M. Moore, J. A. Howington, M. A. Maddaus, R. J. McKenna and D. L. Miller (2012). "Video-Assisted Thoracoscopic Lobectomy Is Less Costly and Morbid Than Open Lobectomy: A Retrospective Multiinstitutional Database Analysis." The Annals of Thoracic Surgery 93(4): 10271032. DOI: http://dx.doi.org/10.1016/j.athoracsur.2011.06.007.

Swanstrom, L. L., R. Kozarek, P. J. Pasricha, S. Gross, D. Birkett, P.-O. Park, V. Saadat, R. Ewers and P. Swain (2005). "Development of a new access device for transgastric surgery." Journal of Gastrointestinal Surgery 9(8): 1129-1137. DOI: 10.1016/j.gassur.2005.08.005.

Szykman, S., J. Racz and R. Sriram (1999). The Representation of Function in ComputerBased Design. ASME Design Engineering Technical Conferences \& Computers and Information in Engineering Conference. Las Vegas, NV. DETC99/DTM-8742.

Trotti, A., A. D. Colevas, A. Setser, V. Rusch, D. Jaques, V. Budach, C. Langer, B. Murphy, R. Cumberlin, C. N. Coleman and P. Rubin (2003). "CTCAE v3.0: development of a comprehensive grading system for the adverse effects of cancer treatment." Seminars in Radiation Oncology 13(3): 176-181. DOI: http://dx.doi.org/10.1016/S1053-4296(03)00031-6.

Tucker, R. D. and C. R. Voyles (1995). "Laparoscopic electrosurgical complications and their prevention." Aorn j 62(1): 51-53, 55, 58-59 passim; quiz 74-57.

Tumer, I. Y. and R. B. Stone (2003). "Analytical Methods for Mapping Function to Failure During High-Risk Component Development." Research in Engineering Design 14(1): 25-33.

van Det, M. J., W. J. H. J. Meijerink, C. Hoff, E. R. Totté and J. P. E. N. Pierie (2009). "Optimal ergonomics for laparoscopic surgery in minimally invasive surgery suites: a review and guidelines." Surgical Endoscopy 23(6): 1279-1285. DOI:

10.1007/s00464-008-0148-x. 
Van Slycke, S., J.-P. Gillardin, K. Van Den Heede, J. Minguet, H. Vermeersch and N. Brusselaers (2016). "Comparison of the harmonic focus and the thunderbeat for open thyroidectomy." Langenbeck's Archives of Surgery 401(6): 851-859. DOI: 10.1007/s00423-016-1448-6.

Voorhis, C. R. W. V. and B. L. Morgan (2007). "Understanding Power and Rules of Thumb for Determining Sample Size." Tutorials in Quantitative Methods for Pschology 3(2): 43-50. DOI: 10.20982/tqmp.03.2.p043.

Wang, K. and A. P. Advincula (2007). "“Current thoughts" in electrosurgery." International Journal of Gynecology \& Obstetrics 97(3): 245-250. DOI: http://dx.doi.org/10.1016/j.ijgo.2007.03.001.

Wang, Z. (2015). "The theoretical basis of minimally-invasive and non-invasive medicine: Treatments-Minimize harm to patients." Ultrasonics Sonochemistry 27: 649-653. DOI: http://dx.doi.org/10.1016/j.ultsonch.2015.05.023.

Watanabe, Y., Y. Kurashima, A. Madani, L. S. Feldman, M. Ishida, A. Oshita, T. Naitoh, K. Noma, K. Yasumasa, H. Nagata, F. Nakamura, K. Ono, Y. Suzuki, N. Matsuhashi, T. Shichinohe and S. Hirano (2016). "Surgeons have knowledge gaps in the safe use of energy devices: a multicenter cross-sectional study." Surgical Endoscopy 30(2): 588-592. DOI: 10.1007/s00464-015-4243-5.

Yilmaz, S., S. R. Daly, C. M. Seifert and R. Gonzalez (2016). "Evidence-based design heuristics for idea generation." Design Studies 46: 95-124. DOI: http://dx.doi.org/10.1016/j.destud.2016.05.001.

Yilmaz, S. and C. M. Seifert (2011). "Creativity through design heuristics: A case study of expert product design." Design Studies 32(4): 384-415. DOI: http://dx.doi.org/10.1016/j.destud.2011.01.003.

Yock, P., S. Zenios, J. Makower, R. Brinton, U. Kumar, F. Watkins, L. Denend, T. Krummel and C. Kurihara (2015). Biodesign: The Process of Inovating Medical Technologies, Cambridge University Press.

Zingg, U., A. McQuinn, D. DiValentino, A. J. Esterman, J. R. Bessell, S. K. Thompson, G. G. Jamieson and D. I. Watson (2009). "Minimally Invasive Versus Open Esophagectomy for Patients With Esophageal Cancer." The Annals of Thoracic Surgery 87(3): 911-919. DOI: http://dx.doi.org/10.1016/j.athoracsur.2008.11.060.

Zuckerman, D. M., P. Brown and S. E. Nissen (2011). "Medical device recalls and the fda approval process." Archives of Internal Medicine 171(11): 1006-1011. DOI: 10.1001/archinternmed.2011.30. 


\author{
CURRICULUM VITAE \\ Marie K. Riggs \\ Department of Mechanical Engineering \\ University of Louisville \\ Louisville, KY 40292 \\ (502) 852-6331 \\ marie.riggs@louisville.edu
}

\title{
EDUCATION
}

$\mathrm{PhD}$ in Mechanical Engineering, GPA 4.0

University of Louisville

M. Eng. in Mechanical Engineering, GPA 4.0

University of Louisville

B.S. in Mechanical Engineering, GPA 3.7

University of Louisville

\section{EXPERIENCE}

Academic Positions

Graduate Assistant

Department of Mechanical Engineering

University of Louisville

Graduate Assistant

May 2013 - May 2014

Mechanical Engineering Lab Courses

University of Louisville

Non-Academic Positions

Founder \& Owner

December 2015 - Present

Botanical Processing, LLC

August 2016 - Present 
Founder, Owner, \& Director

Hawaiian Hula \& Ori Tahiti of Louisville, KY

Research \& Development Co-op

Ethicon Endo-Surgery, Johnson \& Johnson

August 2011 - December 2011

Research \& Development Co-op

January 2011 - May 2011

Ethicon Endo-Surgery, Johnson \& Johnson

AWARDS

University of Louisville, Grosscurth PhD Fellowship

2014

\section{RESEARCH PUBLICATIONS}

Bohm, M. R., Nagel, R. L., \& Riggs, M. K. (2017). Utilizing design intent information to aid in the synthesis of multi-domain systems. Computer-Aided Design and Applications, 14(1), 17-27. doi: 10.1080/16864360.2016.1199752

Riggs, M. K., Bohm, M. R., \& Mountain, P. J. (2016). Examining Relationships Between Device Complexity and Failure Modes of Minimally Invasive Surgical Staplers. Paper presented at the ASME 2016 International Mechanical Engineering Congress \& Exposition Phoenix, Arizona.

Riggs, M. K., Mountain, P. J., Nagel, R. L., Bohm, M. R., \& Linsey, J. S. (2016). Knowledge Retention and Scoring Metrics for Functional Modeling in an Engineering Design Context. (50138), V003T004A008. doi: 10.1115/DETC2016-59640

Mountain, P. J., Bohm, M. R., \& Riggs, M. K. (2016). Evaluation of the Techniques to Describe Device Complexity in Pre and Post Design Stages. Paper presented at the ASME 2016 International Mechanical Engineering Congress \& Exposition, Phoenix, Arizona.

Mountain, P. J., Carini, R. M., Bohm, M. R., \& Riggs, M. K. (2016). A Preliminary Study: The Effects of Personal Motivation on Design Quality. (50657), V011T015A002. doi: 10.1115/IMECE2016-66818

Nagel, R. L., Bohm, M. R., Linsey, J. S., \& Riggs, M. K. (2015). Improving Students' Functional Modeling Skills: A Modeling Approach and a Scoring Rubric. Journal of Mechanical Design, 137(5), 051102. doi:10.1115/1.4029585 